[Electronic Supporting Information]

\title{
Catalytic Asymmetric Hydrogenation of Tetrasubstituted Unsaturated Lactams: An Efficient Approach to Enantioenriched 3,4-Disubstituted Piperidines
}

Congcong Yin, ${ }^{+,}$Yingmin Pan, ${ }^{+,}$Xumu Zhang, ${ }^{*,+}$ and Qin Yin ${ }^{*,+}$

${ }^{+}$Guangdong Provincial Key Laboratory of Catalysis, Department of Chemistry, Southern University of Science and Technology, Shenzhen, Guangdong, 518055, China

* Shenzhen Institute of Advanced Technology, University of Chinese Academy of Sciences, Chinese Academy of Sciences, Shenzhen, Guangdong, 518055, China

\section{Contents}

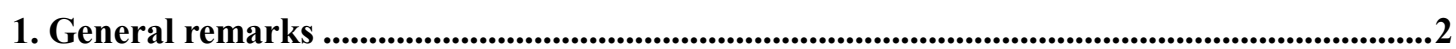

2. General procedures for the synthesis of substrates...............................................................2

3. Detailed optimization of reaction conditions. ................................................................11

4. General procedure for asymmetric hydrogenation.........................................................................13

5. One-pot asymmetric transformations of $1 \mathrm{a}$ to 3 .............................................................................22

6. Scale-up reactions and synthetic applications ……..........................................................................25

7. Monitoring the diastereomeric ratio of the product along with the reaction process ..........26

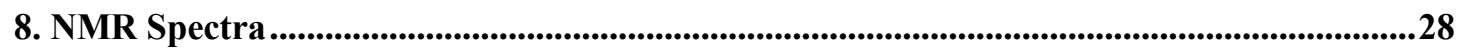

9. Crystal structure of 2c (CCDC 2112114)...........................................................................................67

10. HPLC spectra .....................................................................................................................................69

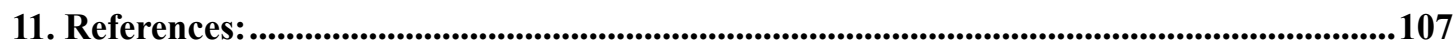




\section{General remarks}

Unless otherwise noted, all experiments were carried out under an atmosphere of argon in a glovebox or using standard Schlenk techniques. Solvents were dried with standard procedures and degassed with argon. Flash column chromatography was performed using Tsingdao silica gel (60, particle size 300-400 mesh). NMR spectra were recorded on a Bruker DPX 400 or a Bruker DPX 600 spectrometer. Chemical shifts $(\delta)$ are reported in ppm and respectively referenced to internal standard $\mathrm{Me}_{4} \mathrm{Si}$ and solvent signals $\left(\mathrm{Me}_{4} \mathrm{Si}, 0\right.$ ppm for ${ }^{1} \mathrm{H} \mathrm{NMR}$ in $\mathrm{CDCl}_{3}$; $77.0 \mathrm{ppm}$ in $\mathrm{CDCl}_{3}$ for ${ }^{13} \mathrm{C} \mathrm{NMR}$ ). Data are reported as follows: chemical shift, multiplicity (s $=$ singlet, $\mathrm{d}=$ doublet, $\mathrm{t}=$ triplet, $\mathrm{q}=$ quartet, hept $=$ heptet, $\mathrm{m}=$ multiplet $)$, coupling constant in hertz $(\mathrm{Hz})$ and signal area integration in natural numbers. HPLC analysis was carried out with Agilent 1260 Series instrument. Optical rotations were measured using a $1 \mathrm{~mL}$ cell with a $1 \mathrm{dm}$ path length on a Rudolph Autopol I polarimeter at $589 \mathrm{~nm}$. Melting points were accessed on a SGWX-4A melting point apparatus, and only the data of suitable crystal products were given. High resolution mass spectra (HRMS) were obtained on Thermo Scientific Q Exactive hybrid quadrupole-Orbitrap mass spectrometer. Substrates 10 was purified by Waters Prep 150 LC System (2545 Binary Gradient Module, 2489 UV/Visible Detector, Waters Fraction Collector III).

\section{General procedures for the synthesis of substrates}

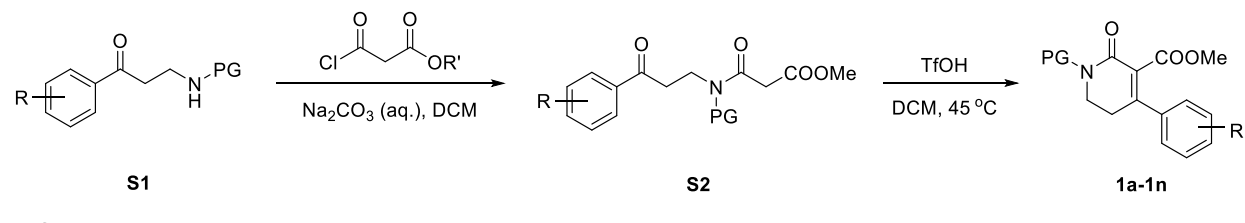

$P G=-P M P,-B n$

Aminoketones S1 were synthesized according to reported methods (S1a-S1d and $\mathbf{S 1 m},{ }^{1,2} \mathbf{S 1 e},{ }^{3-}$ ${ }^{5}$ S1f-S11 and $\left.\mathbf{S 1 o}^{6}\right)$.

To a stirred solution of aminoketone S1 (1.0 equiv) and alkyl 3-chloro-3-oxopropanoate (1.1 equiv) in $\mathrm{CH}_{2} \mathrm{Cl}_{2}(2.75 \mathrm{~mL}$ refers to $1.0 \mathrm{mmol}$ of $\mathbf{S 1})$ was dropwisely added saturated sodium carbonate (1.5 $\mathrm{mL}$ refers to $1 \mathrm{mmol}$ of $\mathbf{S 1}$ ) at RT. Upon completion (monitored by TLC), the reaction mixture was quenched with $\mathrm{H}_{2} \mathrm{O}$. The aqueous layer was separated and extracted twice with $\mathrm{CH}_{2} \mathrm{Cl}_{2}$, and the combined organic layers were dried $\left(\mathrm{Na}_{2} \mathrm{SO}_{4}\right)$ and concentrated in vacuo to afford crude product $\mathbf{S 2}$. The residue was used in the next step without further purification. 
Trifluoromethanesulfonic acid (1.5 equiv) was added to a solution of $\mathbf{S 2}$ (1.0 equiv.) in $\mathrm{CH}_{2} \mathrm{Cl}_{2}$ (10.0 $\mathrm{mL}$ refers to $1.0 \mathrm{mmol}$ of $\mathbf{S 2}$ ), and the mixture was heated to reflux for $24 \mathrm{~h}$ on oil bath. After that, the mixture was cooled to room temperature and quenched with saturated aq. $\mathrm{NaHCO}_{3}$. The mixture was extracted twice with $\mathrm{CH}_{2} \mathrm{Cl}_{2}$, and the organic layers were combined, washed with brine, dried over $\mathrm{Na}_{2} \mathrm{SO}_{4}$, filtered and then concentrated. The residue was purified by flash chromatography to give the desired products 1a-1n (on silica gel, petroleum ether : ethyl acetate $=2: 1$ )

\section{Characterization data of substrates 1a-1o}

\section{Methyl 1-(4-methoxyphenyl)-2-oxo-4-phenyl-1,2,5,6-tetrahydropyridine-3-carboxylate}

(1a)<smiles>COC(=O)C1=C(c2ccccc2)CCN(c2ccc(OC)cc2)C1=O</smiles>

Chemical Formula: $\mathrm{C}_{20} \mathrm{H}_{19} \mathrm{NO}_{4}$

Exact Mass: 337.1314

purified by flash chromatography (on silica gel, petroleum ether : ethyl acetate $=2: 1$ ), white solid (melting point: $155-158{ }^{\circ} \mathrm{C}$ ), $2.63 \mathrm{~g}, 75 \%$ yield in two steps. ${ }^{1} \mathrm{H}$ NMR $\left(400 \mathrm{MHz}, \mathrm{CDCl}_{3}\right) \delta 7.40(\mathrm{br}, 5 \mathrm{H}), 7.28(\mathrm{~d}, J=9.0 \mathrm{~Hz}, 2 \mathrm{H}), 6.91(\mathrm{~d}, J=8.9$ $\mathrm{Hz}, 2 \mathrm{H}), 3.95(\mathrm{t}, J=6.8 \mathrm{~Hz}, 2 \mathrm{H}), 3.80(\mathrm{~s}, 3 \mathrm{H}), 3.68(\mathrm{~s}, 3 \mathrm{H}), 2.96(\mathrm{t}, J=6.8 \mathrm{~Hz}, 2 \mathrm{H}) .{ }^{13} \mathrm{C}\left\{{ }^{1} \mathrm{H}\right\}$ $\operatorname{NMR}\left(101 \mathrm{MHz}, \mathrm{CDCl}_{3}\right) \delta 166.9,161.8,157.3,149.3,137.6,134.9,129.5,128.7,128.0,126.5$, 126.2, 114.1, 55.5, 52.3, 48.0, 29.9. HRMS (ESI)m/z: $[\mathrm{M}+\mathrm{H}]^{+}$Calcd for $\mathrm{C}_{20} \mathrm{H}_{20} \mathrm{NO}_{4}$ 338.1387; found 338.1389 .

Ethyl 1-(4-methoxyphenyl)-2-oxo-4-phenyl-1,2,5,6-tetrahydropyridine-3-carboxylate (1 b)<smiles>CCOC(=O)C1=C(c2ccccc2)CCN(c2ccc(OC)cc2)C1=O</smiles>

Chemical Formula: $\mathrm{C}_{21} \mathrm{H}_{21} \mathrm{NO}_{4}$ Exact Mass: 351.1471 
ether : ethyl acetate $=2: 1$ ), white solid (melting point: $135-139{ }^{\circ} \mathrm{C}$ ), $2.45 \mathrm{~g}, 70 \%$ yield in two steps. ${ }^{1} \mathrm{H}$ NMR $\left(400 \mathrm{MHz}, \mathrm{CDCl}_{3}\right) \delta 7.47-7.36(\mathrm{~m}, 5 \mathrm{H}), 7.34-7.23(\mathrm{~m}, 2 \mathrm{H}), 6.96-6.84(\mathrm{~m}$, 2H), 4.15 (q, $J=7.1 \mathrm{~Hz}, 2 \mathrm{H}), 3.95(\mathrm{t}, J=6.8 \mathrm{~Hz}, 2 \mathrm{H}), 3.80(\mathrm{~s}, 3 \mathrm{H}), 2.95(\mathrm{t}, J=6.8 \mathrm{~Hz}, 2 \mathrm{H})$, $1.11(\mathrm{t}, J=7.1 \mathrm{~Hz}, 3 \mathrm{H}) .{ }^{13} \mathrm{C}\left\{{ }^{1} \mathrm{H}\right\} \mathrm{NMR}\left(101 \mathrm{MHz}, \mathrm{CDCl}_{3}\right) \delta 166.3,161.8,157.7,149.1,137.7$, 134.9, 129.4, 128.6, 128.3, 126.6, 126.2, 114.1, 61.4, 55.4, 48.0, 29.9, 13.8. HRMS (ESI)m/z: $[\mathrm{M}+\mathrm{H}]^{+}$Calcd for $\mathrm{C}_{21} \mathrm{H}_{22} \mathrm{NO}_{4} \quad 352.1543$; found 352.1544.Methyl 1,4-bis(4methoxyphenyl)-2-oxo-1,2,5,6-tetrahydropyridine-3-carboxylate (1c)<smiles>COC(=O)C1=C(c2ccc(OC)cc2)CCN(c2ccc(OC)cc2)C1=O</smiles>

Chemical Formula: $\mathrm{C}_{21} \mathrm{H}_{21} \mathrm{NO}_{5}$

Exact Mass: 367.1420

purified by flash chromatography (on silica gel, petroleum ether : ethyl acetate $=1: 1$ ), white solid (melting point: $154-157^{\circ} \mathrm{C}$ ), $1.33 \mathrm{~g}, 38 \%$ yield in two steps. ${ }^{1} \mathrm{H}$ NMR (400 MHz, $\left.\mathrm{CDCl}_{3}\right) \delta 7.37(\mathrm{~d}, J=8.7 \mathrm{~Hz}, 2 \mathrm{H}), 7.31-7.22(\mathrm{~m}, 2 \mathrm{H})$, $6.92-6.89(\mathrm{dd}, J=8.5,6.2 \mathrm{~Hz}, 4 \mathrm{H}), 3.92(\mathrm{t}, J=6.8 \mathrm{~Hz}, 2 \mathrm{H}), 3.83(\mathrm{~s}, 3 \mathrm{H}), 3.80(\mathrm{~s}, 3 \mathrm{H}), 3.72$ (s, 3H), $2.93(\mathrm{t}, J=6.8 \mathrm{~Hz}, 2 \mathrm{H}) .{ }^{13} \mathrm{C}\left\{{ }^{1} \mathrm{H}\right\} \mathrm{NMR}\left(151 \mathrm{MHz}, \mathrm{CDCl}_{3}\right) \delta$ 167.4, 162.0, 160.6, 157.7, $148.8,134.9,129.7,128.2,126.7,126.1,114.1,114.1,55.4,55.3,52.3,48.0,29.8$. HRMS (ESI)m/z: [M+H] $]^{+}$Calcd for $\mathrm{C}_{21} \mathrm{H}_{22} \mathrm{NO}_{5} 368.1492$; found 368.1495.

Methyl 4-(4-fluorophenyl)-1-(4-methoxyphenyl)-2-oxo-1,2,5,6-tetrahydropyridine-3carboxylate (1d)<smiles>COC(=O)C1=C(c2ccc(F)cc2)CCN(c2ccc(OC)cc2)C1=O</smiles>

Chemical Formula: $\mathrm{C}_{20} \mathrm{H}_{18} \mathrm{FNO}_{4}$

Exact Mass: 355.1220

purified by flash chromatography (on silica gel, petroleum ether : ethyl acetate $=3: 1$ ), white solid (melting point: $158-163{ }^{\circ} \mathrm{C}$ ), $1.4 \mathrm{~g}, 41 \%$ yield in two steps. ${ }^{1} \mathrm{H}$ NMR (400 MHz, $\left.\mathrm{CDCl}_{3}\right) \delta 7.41-7.39$ (m, 2H), $7.30-7.23(\mathrm{~m}, 2 \mathrm{H}), 7.09$ (t, $J=8.5$ $\mathrm{Hz}, 2 \mathrm{H}), 6.95-6.87(\mathrm{~m}, 2 \mathrm{H}), 3.94(\mathrm{t}, J=6.8 \mathrm{~Hz}, 2 \mathrm{H}), 3.80(\mathrm{~s}, 3 \mathrm{H}), 3.69(\mathrm{~s}, 3 \mathrm{H}), 2.92$ (t, $J=$ 
$6.8 \mathrm{~Hz}, 2 \mathrm{H}) .{ }^{19} \mathrm{~F} \mathrm{NMR}\left(565 \mathrm{MHz}, \mathrm{CDCl}_{3}\right) \delta-110.72 .{ }^{13} \mathrm{C}\left\{{ }^{1} \mathrm{H}\right\} \mathrm{NMR}\left(151 \mathrm{MHz}, \mathrm{CDCl}_{3}\right) \delta 166.8$, $163.2(\mathrm{~d}, J=250.5 \mathrm{~Hz}), 161.6,157.7,148.1,134.7,133.6$ (d, $J=3.6 \mathrm{~Hz}), 128.6$ (d, $J=8.5 \mathrm{~Hz})$, 128.1, 126.1, $115.8(\mathrm{~d}, J=21.8 \mathrm{~Hz}), 114.1,55.4,52.3,47.9,29.9$. HRMS (ESI)m/z: $[\mathrm{M}+\mathrm{H}]^{+}$ Calcd for $\mathrm{C}_{20} \mathrm{H}_{19} \mathrm{FNO}_{4} 356.1293$; found 356.1295.

Methyl 1-(4-methoxyphenyl)-2-oxo-4-(m-tolyl)-1,2,5,6-tetrahydropyridine-3-carboxylate (1e)<smiles>COc1ccc(N2CCC(c3cccc(C)c3)=C(C(C)=O)C2=O)cc1</smiles>

Chemical Formula: $\mathrm{C}_{21} \mathrm{H}_{21} \mathrm{NO}_{4}$ Exact Mass: 351.1471

purified by flash chromatography (on silica gel, petroleum ether : ethyl acetate $=2: 1$ ), white solid (melting point: $152-157^{\circ} \mathrm{C}$ ), $0.7 \mathrm{~g}, 67 \%$ yield in two steps. ${ }^{1} \mathrm{H}$ NMR (400 MHz, $\left.\mathrm{CDCl}_{3}\right) \delta 7.31-7.15(\mathrm{~m}, 6 \mathrm{H}), 6.93-6.87(\mathrm{~m}, 2 \mathrm{H}), 3.94(\mathrm{t}, J=6.8$ $\mathrm{Hz}, 2 \mathrm{H}), 3.80$ (s, 3H), 3.69 (s, 3H), 2.94 (t, $J=6.8 \mathrm{~Hz}, 2 \mathrm{H}), 2.37$ (s, 3H). ${ }^{13} \mathrm{C}\left\{{ }^{1} \mathrm{H}\right\}$ NMR $(151$ $\left.\mathrm{MHz}, \mathrm{CDCl}_{3}\right) \delta 166.9,161.8,157.7,149.5,138.4,137.6,134.9,130.9,128.5,127.8,127.1$, 126.1, 123.6, 114.0, 55.4, 52.2, 48.0, 29.9, 21.4. HRMS (ESI)m/z: $[\mathrm{M}+\mathrm{H}]^{+}$Calcd for $\mathrm{C}_{21} \mathrm{H}_{22} \mathrm{NO}_{4} \quad 352.1543$; found 352.1546 .

\section{Methyl 1-benzyl-2-oxo-4-phenyl-1,2,5,6-tetrahydropyridine-3-carboxylate (1f)}<smiles>COC(=O)C1=C(c2ccccc2)CCN(Cc2ccccc2)C1=O</smiles>

Chemical Formula: $\mathrm{C}_{20} \mathrm{H}_{19} \mathrm{NO}_{3}$

Exact Mass: 321.1365

purified by flash chromatography (on silica gel, petroleum ether : ethyl acetate $=2: 1$ ), white solid (melting point: $122-126^{\circ} \mathrm{C}$ ), $1.0 \mathrm{~g}, 62 \%$ yield in two steps. ${ }^{1} \mathrm{H}$ NMR (400 MHz, $\left.\mathrm{CDCl}_{3}\right) \delta 7.41-7.26(\mathrm{~m}, 10 \mathrm{H}), 4.67$ (s, 2H), 3.69 (s, 3H), 3.46 (t, $J$ $=7.0 \mathrm{~Hz}, 2 \mathrm{H}), 2.75(\mathrm{t}, J=7.0 \mathrm{~Hz}, 2 \mathrm{H}) .{ }^{13} \mathrm{C}\left\{{ }^{1} \mathrm{H}\right\} \mathrm{NMR}\left(101 \mathrm{MHz}, \mathrm{CDCl}_{3}\right) \delta 167.0,162.1,148.9$, 137.7, 136.9, 129.3, 128.7, 128.6, 128.2, 127.6, 127.3, 126.4, 52.3, 49.8, 43.7, 29.4. HRMS (ESI)m/z: $[\mathrm{M}+\mathrm{H}]^{+}$Calcd for $\mathrm{C}_{20} \mathrm{H}_{20} \mathrm{NO}_{3}$ 322.1438; found 322.1435. 
(1g)<smiles>COC(=O)C1=C(c2ccc(OC)cc2)CCN(Cc2ccccc2)C1=O</smiles>

Chemical Formula: $\mathrm{C}_{21} \mathrm{H}_{21} \mathrm{NO}_{4}$

Exact Mass: 351.1471 purified by flash chromatography (on silica gel, petroleum ether : ethyl acetate $=1: 1)$, yellow oil, $1.4 \mathrm{~g}, 51 \%$ yield in two steps. ${ }^{1} \mathrm{H} \mathrm{NMR}\left(400 \mathrm{MHz}, \mathrm{CDCl}_{3}\right)$ $\delta 7.34-7.28(\mathrm{~m}, 7 \mathrm{H}), 6.91-6.85(\mathrm{~m}, 2 \mathrm{H}), 4.65(\mathrm{~s}, 2 \mathrm{H}), 3.80(\mathrm{~s}, 3 \mathrm{H}), 3.72(\mathrm{~s}, 3 \mathrm{H}), 3.43(\mathrm{t}, J=$ $7.0 \mathrm{~Hz}, 2 \mathrm{H}), 2.72(\mathrm{t}, J=7.0 \mathrm{~Hz}, 2 \mathrm{H}) .{ }^{13} \mathrm{C}\left\{{ }^{1} \mathrm{H}\right\} \mathrm{NMR}\left(101 \mathrm{MHz}, \mathrm{CDCl}_{3}\right) \delta 167.5,162.3,160.5$, $148.3,137.0,129.8,128.6,128.2,128.1,127.5,126.0,114.0,55.2,52.3,49.7,43.7,29.3$. HRMS (ESI)m/z: $[\mathrm{M}+\mathrm{H}]^{+}$Calcd for $\mathrm{C}_{21} \mathrm{H}_{22} \mathrm{NO}_{4} 352.1543$; found 352.1540 .

\section{Methyl 1-benzyl-4-(3-methoxyphenyl)-2-oxo-1,2,5,6-tetrahydropyridine-3-carboxylate}

(1h)<smiles>COC(=O)C1=C(c2cccc(OC)c2)CCN(Cc2ccccc2)C1=O</smiles>

Chemical Formula: $\mathrm{C}_{21} \mathrm{H}_{21} \mathrm{NO}_{4}$

Exact Mass: 351.1471

purified by flash chromatography (on silica gel, petroleum ether : ethyl acetate $=2: 1)$, yellow oil, $1.2 \mathrm{~g}, 34 \%$ yield. ${ }^{1} \mathrm{H}$ NMR $\left(400 \mathrm{MHz}, \mathrm{CDCl}_{3}\right) \delta 7.37-$ $7.27(\mathrm{~m}, 6 \mathrm{H}), 6.93-6.86(\mathrm{~m}, 3 \mathrm{H}), 4.66(\mathrm{~s}, 2 \mathrm{H}), 3.78(\mathrm{~s}, 3 \mathrm{H}), 3.71(\mathrm{~s}, 3 \mathrm{H}), 3.45(\mathrm{t}, J=7.0 \mathrm{~Hz}$, 2H), $2.74(\mathrm{t}, J=7.0 \mathrm{~Hz}, 2 \mathrm{H}) .{ }^{13} \mathrm{C}\left\{{ }^{1} \mathrm{H}\right\} \mathrm{NMR}\left(151 \mathrm{MHz}, \mathrm{CDCl}_{3}\right) \delta 167.0,162.0,159.5,148.6$, 139.0, 136.9, 129.7, 128.6, 128.2, 127.5, 127.3, 118.7, 115.0, 111.9, 55.2, 52.3, 49.7, 43.7, 29.3. HRMS (ESI)m/z: $[\mathrm{M}+\mathrm{H}]^{+}$Calcd for $\mathrm{C}_{21} \mathrm{H}_{22} \mathrm{NO}_{4}$ 352.1543; found 352.1539. 
Methyl 1-benzyl-4-(4-fluorophenyl)-2-oxo-1,2,5,6-tetrahydropyridine-3-carboxylate (1i)<smiles>COC(=O)C1=C(c2ccc(F)cc2)CCN(Cc2ccccc2)C1=O</smiles>

Chemical Formula: $\mathrm{C}_{20} \mathrm{H}_{18} \mathrm{FNO}_{3}$

Exact Mass: 339.1271

purified by flash chromatography (on silica gel, petroleum

ether : ethyl acetate $=1: 1)$, yellow oil, $1.0 \mathrm{~g}, 42 \%$ yield in two steps. ${ }^{1} \mathrm{H}$ NMR $\left(400 \mathrm{MHz}, \mathrm{CDCl}_{3}\right)$ $\delta 7.48-7.22(\mathrm{~m}, 7 \mathrm{H}), 7.05(\mathrm{t}, J=8.6 \mathrm{~Hz}, 2 \mathrm{H}), 4.65(\mathrm{~s}, 2 \mathrm{H}), 3.69(\mathrm{~s}, 3 \mathrm{H}), 3.45$ (t, $J=7.0 \mathrm{~Hz}$, 2H), $2.71(\mathrm{t}, J=7.0 \mathrm{~Hz}, 2 \mathrm{H}) .{ }^{19} \mathrm{~F}$ NMR $\left(376 \mathrm{MHz}, \mathrm{CDCl}_{3}\right) \delta-110.92 .{ }^{13} \mathrm{C}\left\{{ }^{1} \mathrm{H}\right\} \mathrm{NMR}(151 \mathrm{MHz}$, $\left.\mathrm{CDCl}_{3}\right) \delta 167.0,163.1(\mathrm{~d}, J=250.2 \mathrm{~Hz}), 162.0,147.6,136.9,133.8(\mathrm{~d}, J=3.5 \mathrm{~Hz}), 128.7$, $128.5(\mathrm{~d}, J=8.5 \mathrm{~Hz}), 128.3,127.7,127.5,115.8(\mathrm{~d}, J=21.8 \mathrm{~Hz}), 52.4,49.8,43.7,29.5$. HRMS (ESI)m/z: $[\mathrm{M}+\mathrm{H}]^{+}$Calcd for $\mathrm{C}_{20} \mathrm{H}_{19} \mathrm{FNO}_{3}$ 340.1343; found 340.1341 .

Methyl 1-benzyl-4-(3-fluorophenyl)-2-oxo-1,2,5,6-tetrahydropyridine-3-carboxylate (1j)<smiles>COC(=O)C1=C(c2cccc(F)c2)CCN(Cc2ccccc2)C1=O</smiles>

Chemical Formula: $\mathrm{C}_{20} \mathrm{H}_{18} \mathrm{FNO}_{3}$

Exact Mass: 339.1271

purified by flash chromatography (on silica gel, petroleum ether : ethyl acetate $=2: 1)$, yellow oil, $1.0 \mathrm{~g}, 29 \%$ yield in two steps. ${ }^{1} \mathrm{H} \mathrm{NMR}\left(400 \mathrm{MHz}, \mathrm{CDCl}_{3}\right)$ $\delta 7.37-7.30(\mathrm{~m}, 6 \mathrm{H}), 7.16-7.00(\mathrm{~m}, 3 \mathrm{H}), 4.66(\mathrm{~s}, 2 \mathrm{H}), 3.71(\mathrm{~s}, 3 \mathrm{H}), 3.46(\mathrm{t}, J=7.0 \mathrm{~Hz}, 2 \mathrm{H})$, $2.72(\mathrm{t}, J=7.0 \mathrm{~Hz}, 2 \mathrm{H}) \cdot{ }^{19} \mathrm{~F}$ NMR $\left(376 \mathrm{MHz}, \mathrm{CDCl}_{3}\right) \delta-111.75 .{ }^{13} \mathrm{C}\left\{{ }^{1} \mathrm{H}\right\} \mathrm{NMR}(101 \mathrm{MHz}$, $\left.\mathrm{CDCl}_{3}\right) \delta 166.6,162.6(\mathrm{~d}, J=247.6 \mathrm{~Hz}), 161.8,147.2,139.8(\mathrm{~d}, J=7.6 \mathrm{~Hz}), 136.8,130.3(\mathrm{~d}, J$ $=8.3 \mathrm{~Hz}), 128.7,128.2,128.0,127.6,122.2(\mathrm{~d}, J=3.1 \mathrm{~Hz}), 116.3(\mathrm{~d}, J=21.0 \mathrm{~Hz}), 113.6(\mathrm{~d}, J$ $=22.7 \mathrm{~Hz}), 52.4,49.8,43.6,29.2$. HRMS $(\mathrm{ESI}) \mathrm{m} / \mathrm{z}:[\mathrm{M}+\mathrm{H}]^{+}$Calcd for $\mathrm{C}_{20} \mathrm{H}_{19} \mathrm{FNO}_{3} 340.1343$ found 340.1340 
Methyl 1-benzyl-4-(3-bromophenyl)-2-oxo-1,2,5,6-tetrahydropyridine-3-carboxylate (1k)<smiles>COC(=O)C1=C(c2cccc(Br)c2)CCN(Cc2ccccc2)C1=O</smiles>

Chemical Formula:

$\mathrm{C}_{20} \mathrm{H}_{18} \mathrm{BrNO}_{3}$

Exact Mass: 399.0470

purified by flash chromatography (on silica gel, petroleum ether : ethyl acetate $=2: 1$ ), white solid (melting point: $127-132{ }^{\circ} \mathrm{C}$ ), $1.2 \mathrm{~g}, 31 \%$ yield in two steps. ${ }^{1} \mathrm{H}$ NMR (400 MHz, $\left.\mathrm{CDCl}_{3}\right) \delta 7.48(\mathrm{~m}, 2 \mathrm{H}), 7.38-7.21(\mathrm{~m}, 7 \mathrm{H}), 4.66(\mathrm{~s}, 2 \mathrm{H}), 3.72(\mathrm{~s}, 3 \mathrm{H}), 3.45$ (t, $J=7.0 \mathrm{~Hz}, 2 \mathrm{H}), 2.71(\mathrm{t}, J=7.0 \mathrm{~Hz}, 2 \mathrm{H}) .{ }^{13} \mathrm{C}\left\{{ }^{1} \mathrm{H}\right\} \mathrm{NMR}\left(101 \mathrm{MHz}, \mathrm{CDCl}_{3}\right) \delta$ 166.6, 161.7, 147.0, 139.7, 136.7, 132.3, 130.1, 129.4, 128.7, 128.2, 128.1, 127.6, 125.1, 122.7, 52.4, 49.8, 43.6, 29.2. HRMS (ESI)m/z: $[\mathrm{M}+\mathrm{H}]^{+}$Calcd for $\mathrm{C}_{20} \mathrm{H}_{19} \mathrm{BrNO}_{3} 400.0543$ found 400.0549

Methyl 1-benzyl-2-oxo-4-(thiophen-2-yl)-1,2,5,6-tetrahydropyridine-3-carboxylate (11)<smiles>COC(=O)C1=C(c2cccs2)CCN(Cc2ccccc2)C1=O</smiles>

Chemical Formula: $\mathrm{C}_{18} \mathrm{H}_{17} \mathrm{NO}_{3} \mathrm{~S}$

Exact Mass: 327.0929

purified by flash chromatography (on silica gel, petroleum ether : ethyl acetate $=1: 1$ ), white solid (melting point: $125-129^{\circ} \mathrm{C}$ ), $1.1 \mathrm{~g}, 39 \%$ yield in two steps. ${ }^{1} \mathrm{H}$ NMR $\left(600 \mathrm{MHz}, \mathrm{CDCl}_{3}\right) \delta 7.37(\mathrm{~d}, J=5.0 \mathrm{~Hz}, 1 \mathrm{H}), 7.27-7.18(\mathrm{~m}, 6 \mathrm{H}), 7.05-6.86$ $(\mathrm{m}, 1 \mathrm{H}), 4.56(\mathrm{~s}, 2 \mathrm{H}), 3.81(\mathrm{~s}, 3 \mathrm{H}), 3.36(\mathrm{t}, J=7.0 \mathrm{~Hz}, 2 \mathrm{H}), 2.74(\mathrm{t}, J=7.0 \mathrm{~Hz}, 2 \mathrm{H}) .{ }^{13} \mathrm{C}\left\{{ }^{1} \mathrm{H}\right\}$ $\operatorname{NMR}\left(101 \mathrm{MHz}, \mathrm{CDCl}_{3}\right) \delta 167.3,162.2,139.3,138.8,136.8,129.1,128.6,128.5,128.2,127.9$, 127.5, 124.0, 52.7, 49.7, 43.5, 29.0. HRMS (ESI)m/z: $[\mathrm{M}+\mathrm{H}]^{+}$Calcd for $\mathrm{C}_{18} \mathrm{H}_{18} \mathrm{NO}_{3} \mathrm{~S}$ 328.1002; found 328.1005. 
Mthyl 1-(4-methoxyphenyl)-4-methyl-2-oxo-1,2,5,6-tetrahydropyridine-3-carboxylate

(1m)<smiles>COC(=O)C1=C(C)CCN(c2ccc(OC)cc2)C1=O</smiles>

Chemical Formula: $\mathrm{C}_{15} \mathrm{H}_{17} \mathrm{NO}_{4}$ Exact Mass: 275.1158

purified by flash chromatography (on silica gel, petroleum ether : ethyl acetate $=3: 1)$, yellow oil, $1.6 \mathrm{~g}, 59 \%$ yield in two steps. ${ }^{1} \mathrm{H}$ NMR $\left(600 \mathrm{MHz}, \mathrm{CDCl}_{3}\right)$ $\delta 7.16-7.11(\mathrm{~m}, 2 \mathrm{H}), 6.81(\mathrm{~d}, J=8.9 \mathrm{~Hz}, 2 \mathrm{H}), 3.77(\mathrm{~s}, 3 \mathrm{H}), 3.73(\mathrm{t}, J=12 \mathrm{~Hz}, 2 \mathrm{H}), 3.72(\mathrm{~s}$, 3H), 2.47 (t, $J=6.8 \mathrm{~Hz}, 2 \mathrm{H}), 1.97(\mathrm{~s}, 3 \mathrm{H}) .{ }^{13} \mathrm{C}\left\{{ }^{1} \mathrm{H}\right\} \mathrm{NMR}\left(151 \mathrm{MHz}, \mathrm{CDCl}_{3}\right) \delta$ 166.7, 161.6, 157.6, 150.5, 135.0, 127.6, 126.2, 114.0, 55.5, 52.3, 47.7, 30.6, 20.9. HRMS (ESI)m/z: $[\mathrm{M}+\mathrm{H}]^{+}$ Calcd for $\mathrm{C}_{15} \mathrm{H}_{18} \mathrm{NO}_{4} 276.1230$; found 276.1233.

Methyl 1-benzyl-4-cyclohexyl-2-oxo-1,2,5,6-tetrahydropyridine-3-carboxylate (1n)<smiles>COC(=O)C1=C(C2CCCCC2)CCN(Cc2ccccc2)C1=O</smiles>

Chemical Formula:

$$
\mathrm{C}_{20} \mathrm{H}_{25} \mathrm{NO}_{3}
$$

Exact Mass: 327.1834

purified by flash chromatography (on silica gel, petroleum ether : ethyl acetate $=3: 1)$, yellow oil, $1.4 \mathrm{~g}, 42 \%$ yield in two steps. ${ }^{1} \mathrm{H} \mathrm{NMR}\left(400 \mathrm{MHz}, \mathrm{CDCl}_{3}\right) \delta$ $7.30(\mathrm{~m}, 5 \mathrm{H}), 4.59$ (s, 2H), $3.86(\mathrm{~s}, 3 \mathrm{H}), 3.27(\mathrm{t}, J=7.0 \mathrm{~Hz}, 2 \mathrm{H}), 2.40-2.33(\mathrm{~m}, 1 \mathrm{H}), 2.30(\mathrm{t}$, $J=7.0 \mathrm{~Hz}, 2 \mathrm{H}), 1.82-1.61(\mathrm{~m}, 5 \mathrm{H}), 1.36-1.07(\mathrm{~m}, 5 \mathrm{H}) .{ }^{13} \mathrm{C}\left\{{ }^{1} \mathrm{H}\right\} \mathrm{NMR}\left(151 \mathrm{MHz}, \mathrm{CDCl}_{3}\right) \delta$ $167.1,162.3,156.6,137.1,128.5,128.1,127.4,125.6,52.2,49.5,43.8,43.2,29.9,25.7,25.6$, 23.8. HRMS (ESI)m/z: $[\mathrm{M}+\mathrm{H}]^{+}$Calcd for $\mathrm{C}_{20} \mathrm{H}_{26} \mathrm{NO}_{3}$ 328.1907; found 328.1911. 
Methyl 1-methyl-2-oxo-4-phenyl-1,2,5,6-tetrahydropyridine-3-carboxylate (10)<smiles>COC(=O)C1=C(c2ccccc2)CCN(C)C1=O</smiles>

Chemical Formula: $\mathrm{C}_{14} \mathrm{H}_{15} \mathrm{NO}_{3}$

Exact Mass: 245.1052

Due to the purification difficulty on the isolation of $\mathbf{1 0}$ from its alkene regio-isomer (caused by the cyclization step), 10 was finally purified by preparation HPLC. Conditions: Waters Prep 150 LC System (OD-H, $20 \mathrm{~mm} \times 250 \mathrm{~mm}, n$-hexane : isopropyl alcohol $=80: 20,10 \mathrm{~mL} / \mathrm{min})$.

Yellow oil, 54 mg. ${ }^{~} \mathrm{H}$ NMR (600 MHz, $\left.\mathrm{CDCl}_{3}\right) \delta 7.46-7.28(\mathrm{~m}, 5 \mathrm{H}), 3.67(\mathrm{~s}, 3 \mathrm{H}), 3.55(\mathrm{t}, J$ $=6.9 \mathrm{~Hz}, 2 \mathrm{H}), 3.05(\mathrm{~s}, 3 \mathrm{H}), 2.83(\mathrm{t}, J=7.0 \mathrm{~Hz}, 2 \mathrm{H}) .{ }^{13} \mathrm{C}\left\{{ }^{1} \mathrm{H}\right\} \mathrm{NMR}\left(151 \mathrm{MHz}, \mathrm{CDCl}_{3}\right) \delta 167.0$, 162.4 $148.5,137.8,129.3,128.6,127.4,126.5,52.3,46.7,34.4,29.2$. HRMS (ESI) m/z: $[\mathrm{M}+\mathrm{H}]^{+}$Calcd for $\mathrm{C}_{14} \mathrm{H}_{16} \mathrm{NO}_{3}$ 246.1125; found 246.1123. 


\section{Detailed optimization of reaction conditions.}

Table S1. Screening of bisphosphine ligands. ${ }^{a}$

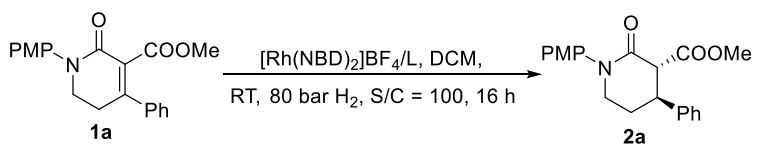

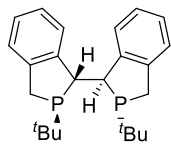

$\left(R_{\mathrm{c}}, S_{\mathrm{p}}\right)$-DuanPhos

NR

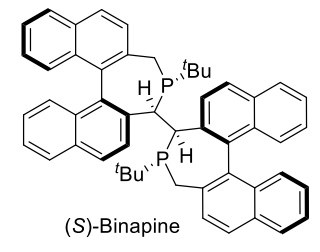

$7 \%$ conv., $72: 18$ dr., $60 \%$ ee

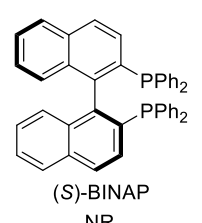

NR

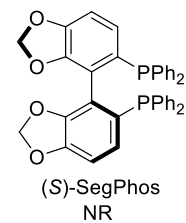

NR

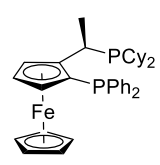

$(R, S)$-JosiPhos

$>99 \%$ conv., $92: 8 \mathrm{dr}$., $67 \%$ ee

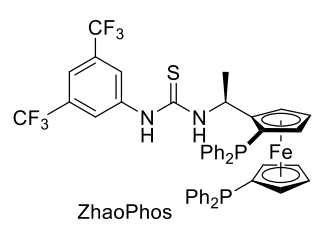

NR

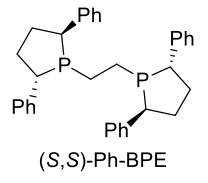

NR

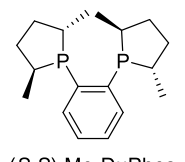

$(S, S)-$ Me-DuPhos

NR

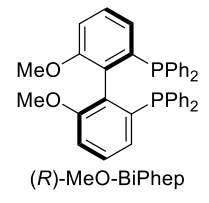

NR

${ }^{a}$ Reaction conditions: [Rh(NBD) $\left.)_{2}\right] \mathrm{BF}_{4} /$ ligand/substrate 1a $(0.05 \mathrm{mmol})$ ratio of $1: 1.1: 100$ in $1 \mathrm{~mL}$ DCM at RT $\left(\sim 25^{\circ} \mathrm{C}\right)$ under 80 bar $\mathrm{H}_{2}$ for $16 \mathrm{~h}$. Conversion and $\mathrm{dr}$ was determined by ${ }^{1} \mathrm{H}$ NMR analysis, ee was determined by HPLC with a chiral stationary phase.

Table S2. Screening of JosiPhos-series ligands. ${ }^{\mathrm{a}}$

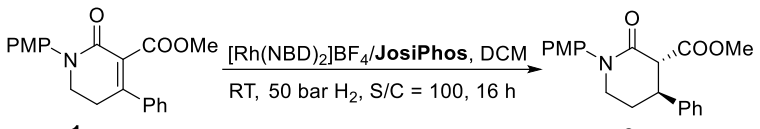

1a

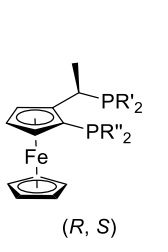

JosiPhos-1: $\mathrm{R}^{\prime}=\mathrm{Cy}, \mathrm{R}^{\prime \prime}=\mathrm{Ph}$

JosiPhos-2: $\mathrm{R}^{\prime}={ }^{\mathrm{t}} \mathrm{Bu}, \mathrm{R} "=\mathrm{Ph}$

JosiPhos-3: $\mathrm{R}^{\prime}=\mathrm{Cy}, \mathrm{R}^{\prime \prime}=3,5-\mathrm{Me}_{2}-4-\mathrm{OMe}-\mathrm{C}_{6} \mathrm{H}_{2}$

JosiPhos-4: $\mathrm{R}^{\prime}=\mathrm{Cy}, \mathrm{R}^{\prime \prime}=3,5{ }^{\mathrm{t}} \mathrm{Bu}_{2}-4-\mathrm{OMe}-\mathrm{C}_{6} \mathrm{H}_{2}$ JosiPhos-5: R' = Cy, R" = 3,5-( $\left(\mathrm{CF}_{3}\right)_{2}-\mathrm{C}_{6} \mathrm{H}_{3}$

JosiPhos-6: R' = 3,5-Me $\mathrm{Me}_{2}-\mathrm{C}_{6} \mathrm{H}_{3}, \mathrm{R}^{\prime \prime}=3,5-\left(\mathrm{CF}_{3}\right)_{2}-\mathrm{C}_{6} \mathrm{H}_{3}$ JosiPhos-7: $\mathrm{R}^{\prime}={ }^{\mathrm{t}} \mathrm{Bu}, \mathrm{R} "={ }^{4}-\mathrm{CF}_{3}-\mathrm{C}_{6} \mathrm{H}_{4}$

JosiPhos-8: $\mathrm{R}^{\prime}=\mathrm{Cy}, \mathrm{R}^{\prime \prime}=\mathrm{Cy}$

JosiPhos-9: $\mathrm{R}^{\prime}=3,5-\mathrm{Me}_{2}-\mathrm{C}_{6} \mathrm{H}_{3}, \mathrm{R}^{\prime \prime}=2$-furanyl JosiPhos-10: $\mathrm{R}^{\prime}=3,5-\mathrm{Me}_{2}-\mathrm{C}_{6} \mathrm{H}_{3}, \mathrm{R}^{\prime \prime}=\mathrm{Ph}$

\begin{tabular}{ccccc}
\hline Entry & $(R, S)-J o s i P h o s$ & Conv. $(\%)$ & dr & Ee (\%) \\
\hline 1 & JosiPhos-1 & 80 & $90: 10$ & 71 \\
2 & JosiPhos-2 & $>99$ & $83: 17$ & 30 \\
$\mathbf{3}$ & JosiPhos-3 & $\mathbf{7 2}$ & $\mathbf{8 7 : 1 3}$ & $\mathbf{7 7}$ \\
4 & JosiPhos-4 & 10 & $89: 11$ & 90 \\
5 & JosiPhos-5 & trace & - & - \\
6 & JosiPhos-6 & trace & - & - \\
7 & JosiPhos-7 & $>99$ & $80: 20$ & 46 \\
8 & JosiPhos-8 & 82 & $94: 6$ & 2 \\
9 & JosiPhos-9 & trace & - & - \\
10 & JosiPhos-10 & trace & - & - \\
\hline
\end{tabular}

${ }^{a}$ Reaction conditions: metal/ligand/substrate 1a $(0.05 \mathrm{mmol})$ ratio of $1: 1.1: 100 \mathrm{in} 1 \mathrm{~mL} \mathrm{DCM}$ at RT $\left(\sim 25^{\circ} \mathrm{C}\right)$ under 50 bar $\mathrm{H}_{2}$ for $16 \mathrm{~h}$. Conversion and $\mathrm{dr}$ was determined by ${ }^{1} \mathrm{H}$ NMR analysis, ee was determined by HPLC with a chiral stationary phase. 
Table S3. Screening of various solvents. ${ }^{\mathrm{a}}$<smiles>COC(=O)c1c(-c2ccccc2)ccn(NC(=O)[O-])c1=O</smiles>

\begin{tabular}{cccccc}
\hline Entry & Solvent & $(R, S)$-Josiphos & Conv. $(\%)$ & $\mathrm{dr}$ & Ee (\%) \\
\hline 1 & DCM & JosiPhos-3 & 72 & $87: 13$ & 77 \\
2 & MeOH & JosiPhos-3 & NR & - & - \\
$\mathbf{3}$ & PhMe & JosiPhos-3 & $\mathbf{1 5}$ & $\mathbf{8 5 : 1 5}$ & $\mathbf{9 3}$ \\
4 & dioxane & JosiPhos-3 & NR & - & - \\
5 & THF & JosiPhos-3 & 31 & $92: 8$ & 89 \\
6 & DCE & JosiPhos-3 & NR & - & - \\
7 & MeOH & JosiPhos-4 & NR & - & - \\
8 & DCM & JosiPhos-4 & 10 & $89: 11$ & 90 \\
9 & PhMe & JosiPhos-4 & trace & - & - \\
10 & dioxane & JosiPhos-4 & trace & - & - \\
11 & THF & JosiPhos-4 & 21 & $96: 4$ & 90 \\
12 & DCE & JosiPhos-4 & 20 & $93: 7$ & 90 \\
\hline
\end{tabular}

${ }^{a}$ Reaction conditions: $[\mathbf{1 a}]=0.05 \mathrm{mmol}, 0.0005 \mathrm{mmol}\left[\mathrm{Rh}(\mathrm{NBD})_{2}\right] \mathrm{BF}_{4}, 0.0011 \mathrm{mmol}$ ligand, solvent volume $=1.0 \mathrm{~mL}$. The catalyst was performed in DCM $(0.1 \mathrm{~mL}$ for each reaction vial $)$ under 50 bar $\mathrm{H}_{2}$ for $16 \mathrm{~h}$. Conversion and dr was determined by ${ }^{1} \mathrm{H}$ NMR analysis, ee was determined by HPLC with a chiral stationary phase.

Table S4. Screening of other conditions. ${ }^{\mathrm{a}}$

\begin{tabular}{|c|c|c|c|c|c|c|c|c|}
\hline Entry & M & $\mathrm{T}\left({ }^{\circ} \mathrm{C}\right)$ & $\begin{array}{c}\mathrm{P} \\
\text { (bar) }\end{array}$ & $\begin{array}{l}\text { Time } \\
\text { (h) }\end{array}$ & $\mathrm{S} / \mathrm{C}$ & $\begin{array}{l}\text { Conv. } \\
(\%)\end{array}$ & $\mathrm{dr}$ & $\begin{array}{c}\text { Ee } \\
(\%) \\
\end{array}$ \\
\hline 1 & {$\left[\mathrm{Rh}(\mathrm{NBD})_{2}\right] \mathrm{BF}_{4}$} & 60 & 50 & 24 & 100 & 72 & $94: 6$ & 90 \\
\hline 2 & {$\left[\mathrm{Rh}(\mathrm{NBD})_{2}\right] \mathrm{BF}_{4}$} & 50 & 50 & 24 & 100 & 72 & $81: 19$ & 90 \\
\hline 3 & {$\left[\mathrm{Rh}(\mathrm{NBD})_{2}\right] \mathrm{BF}_{4}$} & 40 & 50 & 24 & 100 & 42 & $88: 12$ & 92 \\
\hline $4^{b}$ & {$\left[\mathrm{Rh}(\mathrm{NBD})_{2}\right] \mathrm{BF}_{4}$} & 40 & 50 & 24 & 100 & 37 & $86: 14$ & 92 \\
\hline 5 & {$\left[\mathrm{Rh}(\mathrm{NBD})_{2}\right] \mathrm{BF}_{4}$} & 40 & 50 & 24 & 50 & 72 & $85: 15$ & 94 \\
\hline 6 & {$\left[\mathrm{Rh}(\mathrm{NBD})_{2}\right] \mathrm{BF}_{4}$} & 40 & 20 & 24 & 50 & 46 & $86: 14$ & 90 \\
\hline 7 & {$\left[\mathrm{Rh}(\mathrm{NBD})_{2}\right] \mathrm{BF}_{4}$} & 40 & 80 & 24 & 50 & 91 & $79: 21$ & 93 \\
\hline 8 & {$\left[\mathrm{Rh}(\mathrm{NBD})_{2}\right] \mathrm{BF}_{4}$} & 40 & 80 & 48 & 50 & 96 & $90: 10$ & 93 \\
\hline 9 & {$\left[\mathrm{Rh}(\mathrm{NBD})_{2}\right] \mathrm{BF}_{4}$} & 40 & 80 & 72 & 50 & $>99$ & 94:6 & 92 \\
\hline 10 & {$\left[\mathrm{Rh}(\mathrm{COD})_{2}\right] \mathrm{BF}_{4}$} & 40 & 80 & 72 & 50 & 63 & $94: 6$ & 92 \\
\hline 11 & {$[\mathrm{Rh}(\mathrm{COD}) \mathrm{Cl}]_{2}$} & 40 & 80 & 72 & 50 & 17 & $93: 7$ & 10 \\
\hline
\end{tabular}

${ }^{a}$ Reaction conditions: $[\mathbf{1 a}]=0.05 \mathrm{mmol}$, Conversion and $\mathrm{dr}$ was determined by ${ }^{1} \mathrm{H}$ NMR analysis, ee was determined by HPLC with a chiral stationary phase. ${ }^{b} \mathrm{c}=0.1 \mathrm{M}$. 


\section{General procedure for asymmetric hydrogenation.}
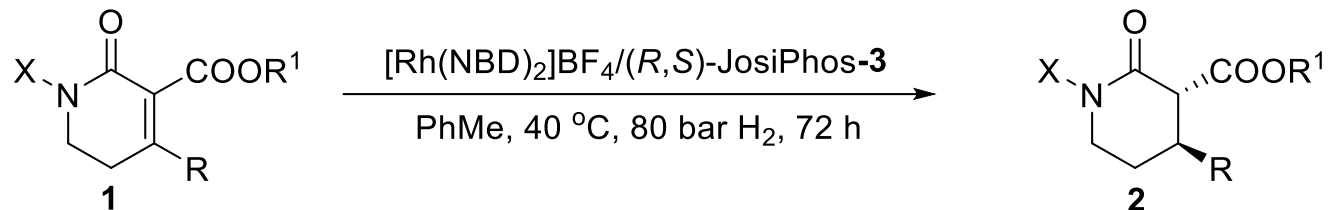

General procedure (at $\mathrm{S} / \mathrm{C}=50$ ): To a $4.0 \mathrm{~mL}$ vial was added the catalyst precursor $\left[\mathrm{Rh}(\mathrm{NBD})_{2}\right] \mathrm{BF}_{4}\left(3.74 \mathrm{mg}, 5 \times 10^{-3} \mathrm{mmol}\right),(R, S)$-JosiPhos-3 $\left(7.8 \mathrm{mg}, 1.1 \times 10^{-2} \mathrm{mmol}\right)$ and anhydrous PhMe (2.0 mL) in an argon-filled glovebox. The mixture was stirred for $0.5 \mathrm{~h}$ at RT, giving orange solution. On the other hand, $1(0.05 \mathrm{mmol})$ was dissolved in anhydrous toluene $(0.8 \mathrm{~mL})$ in a $3 \mathrm{~mL}$ hydrogenation reaction vessel, followed by addition of an aliquot of the catalyst solution $(200 \mu \mathrm{L})$. Then the vessel was placed in an autoclave which was then charged with 80 bar of $\mathrm{H}_{2}$ and stirred at $40{ }^{\circ} \mathrm{C}$ for $72 \mathrm{~h}$. After slowly releasing the hydrogen pressure, the reaction mixture was passed through a short column of silica gel to remove the metal complex. The filtration was concentrated and the resulting residue was purified by a small section of plug tube (neutral $\mathrm{Al}_{2} \mathrm{O}_{3}$ ) with ethyl acetate to afford the desired product. The ee value was determined by chiral HPLC.

Racemic samples were prepared by hydrogenation (50 bar) of 1 with $\mathrm{Pd} / \mathrm{C}$ in methanol.

Notation: Although with tremendous effort, some racemic samples of $\mathbf{2}$ could not be fully resolved by HPLC (typically displaying 3 peaks containing a pair of enantiomers) in our lab. However, this problem does not affect the determination of the ee values of the major transisomers.

\section{Methyl (3R,4S)-1-(4-methoxyphenyl)-2-oxo-4-phenylpiperidine-3-carboxylate (2a)}<smiles>COc1ccc(N2CC[C@H](c3ccccc3)[C@H](C(C)=O)C2=O)cc1</smiles>

Chemical Formula: $\mathrm{C}_{20} \mathrm{H}_{21} \mathrm{NO}_{4}$

Exact Mass: $339.1471 \quad 0.05 \mathrm{mmol}$ scale, yellow solid (melting point: $\left.98-101{ }^{\circ} \mathrm{C}\right), 16.1$ mg, $92 \%$ yield, $92 \%$ ee, $94 / 6 \mathrm{dr},[\alpha]^{25}{ }_{\mathrm{D}}=+7.2\left(c=1.0, \mathrm{CHCl}_{3}\right) .{ }^{1} \mathrm{H} \mathrm{NMR}\left(600 \mathrm{MHz}, \mathrm{CDCl}_{3}\right) \delta$ 7.35 (t, J=7.5 Hz, 2H), $7.29-7.25$ (m, 3H), $7.22-7.18(\mathrm{~m}, 2 \mathrm{H}), 6.97-6.87$ (m, 2H), $3.88-$ $3.83(\mathrm{~m}, 1 \mathrm{H}), 3.81(\mathrm{~s}, 3 \mathrm{H}), 3.76(\mathrm{~d}, J=10.7 \mathrm{~Hz}, 1 \mathrm{H}), 3.62(\mathrm{~s}, 3 \mathrm{H}), 3.66-3.59(\mathrm{~m}, 2 \mathrm{H}), 2.25-$ 
$2.21(\mathrm{~m}, 2 \mathrm{H}) .{ }^{13} \mathrm{C}\left\{{ }^{1} \mathrm{H}\right\} \mathrm{NMR}\left(101 \mathrm{MHz}, \mathrm{CDCl}_{3}\right) \delta 170.4,166.0,158.3,141.4,135.2,128.9$, 127.4, 127.2, 126.8, 114.4, 56.7, 55.5, 52.3, 50.7, 42.5, 29.8. HRMS (ESI)m/z: $[\mathrm{M}+\mathrm{H}]^{+}$Calcd for $\mathrm{C}_{20} \mathrm{H}_{22} \mathrm{NO}_{4} \quad 340.1543$; found 340.1541 .

The enantiomeric excess was determined by UPLC on Daicel Chiralcel IA-U Column (hexane : isopropanol $=85: 15$, flow rate $=0.7 \mathrm{~mL} / \mathrm{min}, \mathrm{T}=25^{\circ} \mathrm{C}, \mathrm{UV}$ detection at $\left.\lambda=210 \mathrm{~nm}\right), \mathrm{t}_{\mathrm{R} 1}=$ $10.30 \mathrm{~min}$ (major), $\mathrm{t}_{\mathrm{R} 2}=14.16 \mathrm{~min}$ (minor).

Ethyl (3R,4S)-1-(4-methoxyphenyl)-2-oxo-4-phenylpiperidine-3-carboxylate (2b)<smiles>CCOC(=O)[C@H]1C(=O)N(c2ccc(OC)cc2)CC[C@@H]1c1ccccc1</smiles>

Chemical Formula: $\mathrm{C}_{21} \mathrm{H}_{23} \mathrm{NO}_{4}$

Exact Mass: 353.1627

$0.05 \mathrm{mmol}$ scale, yellow solid (melting point: $100-108^{\circ} \mathrm{C}$ ), $17.0 \mathrm{mg}, 97 \%$ yield, $92 \%$ ee, $11 / 1 \mathrm{dr},[\alpha]^{25} \mathrm{D}=+8.0\left(c=1.0, \mathrm{CHCl}_{3}\right) .{ }^{1} \mathrm{H} \mathrm{NMR}\left(400 \mathrm{MHz}, \mathrm{CDCl}_{3}\right)$ $\delta 7.38-7.31(\mathrm{~m}, 2 \mathrm{H}), 7.29-7.24(\mathrm{~m}, 3 \mathrm{H}), 7.23-7.17(\mathrm{~m}, 2 \mathrm{H}), 6.95-6.87(\mathrm{~m}, 2 \mathrm{H}), 4.17-$ $4.00(\mathrm{~m}, 2 \mathrm{H}), 3.89-3.83(\mathrm{~m}, 1 \mathrm{H}), 3.80(\mathrm{~s}, 3 \mathrm{H}), 3.73(\mathrm{~d}, J=10.7 \mathrm{~Hz}, 1 \mathrm{H}), 3.67-3.56(\mathrm{~m}, 2 \mathrm{H})$, $2.25-2.20(\mathrm{~m}, 2 \mathrm{H}), 1.10(\mathrm{t}, J=7.1 \mathrm{~Hz}, 3 \mathrm{H}) .{ }^{13} \mathrm{C}\left\{{ }^{1} \mathrm{H}\right\} \mathrm{NMR}\left(101 \mathrm{MHz}, \mathrm{CDCl}_{3}\right) \delta$ 169.9, 166.1, 158.3, 141.4, 135.3, 128.8, 127.3, 127.2, 126.8, 114.4, 61.1, 56.7, 55.4, 50.7, 42.5, 29.8, 14.0. HRMS (ESI)m/z: $[\mathrm{M}+\mathrm{H}]^{+}$Calcd for $\mathrm{C}_{21} \mathrm{H}_{24} \mathrm{NO}_{4}$ 354.1700; found 354.1695.

The enantiomeric excess was determined by UPLC on Daicel Chiralcel IA-U Column (hexane : isopropanol $=85: 15$, flow rate $=0.7 \mathrm{~mL} / \mathrm{min}, \mathrm{T}=25{ }^{\circ} \mathrm{C}$, $\mathrm{UV}$ detection at $\left.\lambda=210 \mathrm{~nm}\right), \mathrm{t}_{\mathrm{R} 1}=$ $8.15 \min$ (major), $\mathrm{t}_{\mathrm{R} 2}=13.74 \min$ (minor).

Methyl (3R,4S)-1,4-bis(4-methoxyphenyl)-2-oxopiperidine-3-carboxylate (2c)<smiles>COc1ccc(C2CCN(c3ccc(OC)cc3)C(=O)[C@H]2C(C)=O)cc1</smiles>

Chemical Formula: $\mathrm{C}_{21} \mathrm{H}_{23} \mathrm{NO}_{5}$

Exact Mass: 369.1576

$0.05 \mathrm{mmol}$ scale, yellow solid (melting point: $105-112{ }^{\circ} \mathrm{C}$ ), $17.1 \mathrm{mg}, 91 \%$ yield, $91 \%$ ee, $16 / 1 \mathrm{dr},[\alpha]^{25} \mathrm{D}=+12.0\left(c=1.0, \mathrm{CHCl}_{3}\right) .{ }^{1} \mathrm{H}$ NMR $(600 \mathrm{MHz}$, 
$\left.\mathrm{CDCl}_{3}\right) \delta 7.19(\mathrm{dd}, J=14.6,8.6 \mathrm{~Hz}, 4 \mathrm{H}), 6.91(\mathrm{~d}, J=8.7 \mathrm{~Hz}, 2 \mathrm{H}), 6.88(\mathrm{~d}, J=8.5 \mathrm{~Hz}, 2 \mathrm{H})$, $3.87-3.82(\mathrm{~m}, 1 \mathrm{H}), 3.80(\mathrm{~s}, 6 \mathrm{H}), 3.73-3.68(\mathrm{~m}, 1 \mathrm{H}), 3.64-3.60(\mathrm{~m}, 1 \mathrm{H}), 3.62(\mathrm{~s}, 3 \mathrm{H}), 3.58$ - $3.52(\mathrm{~m}, 1 \mathrm{H}), 2.20-2.17(\mathrm{~m}, 2 \mathrm{H}) .{ }^{13} \mathrm{C}\left\{{ }^{1} \mathrm{H}\right\} \mathrm{NMR}\left(151 \mathrm{MHz}, \mathrm{CDCl}_{3}\right) \delta$ 170.5, 166.0, 158.7, 158.3, 135.3, 133.5, 127.8, 127.2, 114.4, 114.2, 57.0, 55.5, 55.2, 52.2, 50.7, 41.7, 30.0. HRMS (ESI)m/z: [M+H] ${ }^{+}$Calcd for $\mathrm{C}_{21} \mathrm{H}_{24} \mathrm{NO}_{5} 370.1649$; found 370.1647 .

The enantiomeric excess was determined by UPLC on Daicel Chiralcel OD-3 Column (hexane : isopropanol $=80: 20$, flow rate $=0.5 \mathrm{~mL} / \mathrm{min}, \mathrm{T}=25^{\circ} \mathrm{C}, \mathrm{UV}$ detection at $\left.\lambda=210 \mathrm{~nm}\right), \mathrm{t}_{\mathrm{R} 1}=$ $13.54 \min$ (minor), $\mathrm{t}_{\mathrm{R} 2}=18.31$ min (major).

\section{Methyl (3R,4S)-4-(4-fluorophenyl)-1-(4-methoxyphenyl)-2-oxopiperidine-3-carboxylate} (2d)<smiles>COc1ccc(N2CC[C@H](c3ccc(F)cc3)[C@H](C(C)=O)C2=O)cc1</smiles>

Chemical Formula: $\mathrm{C}_{20} \mathrm{H}_{20} \mathrm{FNO}_{4}$

Exact Mass: 357.1376

$0.05 \mathrm{mmol}$ scale, yellow solid (melting point: $113-119{ }^{\circ} \mathrm{C}$ ), $16.5 \mathrm{mg}, 94 \%$ yield, $91 \%$ ee, $14 / 1 \mathrm{dr},[\alpha]^{25} \mathrm{D}=+17.6\left(c=1.0, \mathrm{CHCl}_{3}\right) .{ }^{1} \mathrm{H}$ NMR $(400 \mathrm{MHz}$, $\left.\mathrm{CDCl}_{3}\right) \delta 7.25-7.18(\mathrm{~m}, 4 \mathrm{H}), 7.04(\mathrm{t}, J=8.6 \mathrm{~Hz}, 2 \mathrm{H}), 6.91(\mathrm{~m}, 2 \mathrm{H}), 3.89-3.81(\mathrm{~m}, 1 \mathrm{H}), 3.80$ (s, 3H), $3.70(\mathrm{~d}, J=10.9 \mathrm{~Hz}, 1 \mathrm{H}), 3.67-3.56(\mathrm{~m}, 2 \mathrm{H}), 3.62(\mathrm{~s}, 3 \mathrm{H}), 2.19(\mathrm{~m}, 2 \mathrm{H}) .{ }^{19} \mathrm{~F}$ NMR $\left(376 \mathrm{MHz}, \mathrm{CDCl}_{3}\right) \delta-115.02 .{ }^{13} \mathrm{C}\left\{{ }^{1} \mathrm{H}\right\} \mathrm{NMR}\left(151 \mathrm{MHz}, \mathrm{CDCl}_{3}\right) \delta 170.2,165.7,161.9(\mathrm{~d}, J=$ $245.9 \mathrm{~Hz}), 158.3,137.2(\mathrm{~d}, J=3.2 \mathrm{~Hz}), 135.1,128.3(\mathrm{~d}, J=7.9 \mathrm{~Hz}), 127.1,115.8(\mathrm{~d}, J=21.3$ $\mathrm{Hz}), 114.4,56.9,55.4,52.3,50.6,41.8,29.9 . \mathrm{HRMS}(\mathrm{ESI}) \mathrm{m} / \mathrm{z}$ : $[\mathrm{M}+\mathrm{H}]^{+}$Calcd for $\mathrm{C}_{20} \mathrm{H}_{21} \mathrm{FNO}_{4}$ 358.1449; found 358.1445.

The enantiomeric excess was determined by HPLC on Daicel Chiralcel OD-3 Column (hexane : isopropanol $=80: 20$, flow rate $=1 \mathrm{~mL} / \mathrm{min}, \mathrm{T}=25^{\circ} \mathrm{C}, \mathrm{UV}$ detection at $\left.\lambda=210 \mathrm{~nm}\right), \mathrm{t}_{\mathrm{R} 1}=$ $28.81 \min$ (minor), $\mathrm{t}_{\mathrm{R} 2}=53.16$ min (major). 
Methyl (3R,4S)-1-(4-methoxyphenyl)-2-oxo-4-(m-tolyl)piperidine-3-carboxylate (2e)<smiles>COc1ccc(N2CC[C@H](c3cccc(C)c3)[C@H](C(C)=O)C2=O)cc1</smiles>

Chemical Formula: $\mathrm{C}_{21} \mathrm{H}_{23} \mathrm{NO}_{4}$

Exact Mass: 353.1627

0.05 mmol scale, yellow solid (melting point: $91-97^{\circ} \mathrm{C}$ ), 17.1

mg, $96 \%$ yield, $83 \%$ ee, $14 / 1 \mathrm{dr},[\alpha]^{25} \mathrm{D}=+11.8\left(c=1.0, \mathrm{CHCl}_{3}\right) .{ }^{1} \mathrm{H}$ NMR $\left(400 \mathrm{MHz}, \mathrm{CDCl}_{3}\right)$ $\delta 7.24-7.17(\mathrm{~m}, 3 \mathrm{H}), 7.06(\mathrm{t}, J=10.2 \mathrm{~Hz}, 3 \mathrm{H}), 6.94-6.89(\mathrm{~m}, 2 \mathrm{H}), 3.88-3.83(\mathrm{~m}, 1 \mathrm{H}), 3.80$ (s, 3H), $3.75(\mathrm{~d}, J=10.6 \mathrm{~Hz}, 1 \mathrm{H}), 3.66-3.53(\mathrm{~m}, 2 \mathrm{H}), 3.62(\mathrm{~s}, 3 \mathrm{H}), 2.35(\mathrm{~s}, 3 \mathrm{H}), 2.21(\mathrm{~m}, 2 \mathrm{H})$. ${ }^{13} \mathrm{C}\left\{{ }^{1} \mathrm{H}\right\}$ NMR (101 MHz, $\left.\mathrm{CDCl}_{3}\right) \delta 170.4,166.0,158.3,141.4,138.5,135.3,128.7,128.1$, 127.5, 127.1, 123.7, 114.4, 56.6, 55.4, 52.3, 50.7, 42.4, 29.8, 21.4. HRMS (ESI)m/z: $[\mathrm{M}+\mathrm{H}]^{+}$ Calcd for $\mathrm{C}_{21} \mathrm{H}_{24} \mathrm{NO}_{4} \quad 354.1700$; found 354.1696.

The enantiomeric excess was determined by HPLC on Daicel Chiralcel OD-3 Column (hexane : isopropanol $=80: 20$, flow rate $=1 \mathrm{~mL} / \mathrm{min}, \mathrm{T}=25^{\circ} \mathrm{C}, \mathrm{UV}$ detection at $\left.\lambda=210 \mathrm{~nm}\right), \mathrm{t}_{\mathrm{R} 1}=$ $25.99 \min$ (minor), $\mathrm{t}_{\mathrm{R} 2}=32.14 \min$ (major).

\section{Methyl (3R,4S)-1-benzyl-2-oxo-4-phenylpiperidine-3-carboxylate (2f)}<smiles>COC(=O)[C@H]1C(=O)N(Cc2ccccc2)CC[C@H]1c1ccccc1</smiles>

Chemical Formula: $\mathrm{C}_{20} \mathrm{H}_{21} \mathrm{NO}_{3}$

Exact Mass: 323.1521

$0.05 \mathrm{mmol}$ scale, yellow oil, $0.98 \mathrm{~g}, 98 \%$ yield, $91 \%$ ee, $15 / 1$ $\mathrm{dr},[\alpha]^{25} \mathrm{D}=+3.4\left(c=1.0, \mathrm{CHCl}_{3}\right) .{ }^{1} \mathrm{H}$ NMR $\left(600 \mathrm{MHz}, \mathrm{CDCl}_{3}\right) \delta 7.34-7.25(\mathrm{~m}, 7 \mathrm{H}), 7.21(\mathrm{t}$, $J=7.3 \mathrm{~Hz}, 1 \mathrm{H}), 7.16(\mathrm{~d}, J=7.6 \mathrm{~Hz}, 2 \mathrm{H}), 4.75(\mathrm{~d}, J=14.5 \mathrm{~Hz}, 1 \mathrm{H}), 4.50(\mathrm{~d}, J=14.5 \mathrm{~Hz}, 1 \mathrm{H})$, $3.64(\mathrm{~d}, J=10.9 \mathrm{~Hz}, 1 \mathrm{H}), 3.59$ (s, 3H), $3.45-3.35$ (m, 2H), $3.27-3.24$ (m, 1H), $2.04-1.94$ (m, 2H). ${ }^{13} \mathrm{C}\left\{{ }^{1} \mathrm{H}\right\}$ NMR $\left(151 \mathrm{MHz}, \mathrm{CDCl}_{3}\right) \delta 170.3,165.6,141.1,136.4,128.6,128.4,127.9$, 127.3, 127.1, 126.5, 56.2, 52.0, 50.1, 45.9, 42.2, 29.0. HRMS (ESI)m/z: $[\mathrm{M}+\mathrm{H}]^{+}$Calcd for $\mathrm{C}_{20} \mathrm{H}_{22} \mathrm{NO}_{3}$ 324.1594; found 324.1591.

The enantiomeric excess was determined by HPLC on Daicel Chiralcel AS-3 Column (hexane : isopropanol $=80: 20$, flow rate $=1 \mathrm{~mL} / \mathrm{min}, \mathrm{T}=25^{\circ} \mathrm{C}$, UV detection at $\left.\lambda=220 \mathrm{~nm}\right), \mathrm{t}_{\mathrm{R} 1}=$ 
$17.50 \min$ (major), $\mathrm{t}_{\mathrm{R} 2}=20.94 \min$ (minor).

\section{Methyl (3R,4S)-1-benzyl-4-(4-methoxyphenyl)-2-oxopiperidine-3-carboxylate (2g)}<smiles>COc1ccc(C2CCN(Cc3ccccc3)C(=O)[C@H]2C(C)=O)cc1</smiles>

Chemical Formula: $\mathrm{C}_{21} \mathrm{H}_{23} \mathrm{NO}_{4}$

Exact Mass: 353.1627

$0.05 \mathrm{mmol}$ scale, yellow oil, $16 \mathrm{mg}, 94 \%$ yield, $89 \%$ ee, $12 / 1$ $\mathrm{dr},[\alpha]^{25} \mathrm{D}=+4.7\left(c=1.0, \mathrm{CHCl}_{3}\right) .{ }^{1} \mathrm{H}$ NMR $\left(400 \mathrm{MHz}, \mathrm{CDCl}_{3}\right) \delta 7.39-7.23(\mathrm{~m}, 5 \mathrm{H}), 7.10(\mathrm{~d}$, $J=8.6 \mathrm{~Hz}, 2 \mathrm{H}), 6.84(\mathrm{~d}, J=8.6 \mathrm{~Hz}, 2 \mathrm{H}), 4.79(\mathrm{~d}, J=14.5 \mathrm{~Hz}, 1 \mathrm{H}), 4.49(\mathrm{~d}, J=14.5 \mathrm{~Hz}, 1 \mathrm{H})$, $3.78(\mathrm{~s}, 3 \mathrm{H}), 3.64(\mathrm{~s}, 3 \mathrm{H}), 3.60(\mathrm{~d}, J=10.9 \mathrm{~Hz}, 1 \mathrm{H}), 3.45-3.34(\mathrm{~m}, 2 \mathrm{H}), 3.31-3.24(\mathrm{~m}, 1 \mathrm{H})$, $2.06-1.92(\mathrm{~m}, 2 \mathrm{H}) .{ }^{13} \mathrm{C}\left\{{ }^{1} \mathrm{H}\right\} \mathrm{NMR}\left(101 \mathrm{MHz}, \mathrm{CDCl}_{3}\right) \delta 170.7,165.9,158.7,136.6,133.4$, 128.7, 128.2, 127.7, 127.6, 114.2, 56.7, 55.2, 52.3, 50.3, 46.2, 41.6, 29.4. HRMS (ESI)m/z: $[\mathrm{M}+\mathrm{H}]^{+}$Calcd for $\mathrm{C}_{21} \mathrm{H}_{24} \mathrm{NO}_{4}$ 354.1700; found 354.1703.

The enantiomeric excess was determined by HPLC on Daicel Chiralcel AS-3 Column (hexane : isopropanol $=85: 15$, flow rate $=1 \mathrm{~mL} / \mathrm{min}, \mathrm{T}=25^{\circ} \mathrm{C}, \mathrm{UV}$ detection at $\left.\lambda=220 \mathrm{~nm}\right), \mathrm{t}_{\mathrm{R} 1}=$ $42.99 \min$ (major), $\mathrm{t}_{\mathrm{R} 2}=50.75 \min$ (minor).

\section{Methyl (3R,4S)-1-benzyl-4-(3-methoxyphenyl)-2-oxopiperidine-3-carboxylate (2h)}<smiles>COC(=O)[C@H]1C(=O)N(Cc2ccccc2)CC[C@H]1c1cccc(OC)c1</smiles>

Chemical Formula: $\mathrm{C}_{21} \mathrm{H}_{23} \mathrm{NO}_{4}$

Exact Mass: 353.1627

$0.05 \mathrm{mmol}$ scale, yellow oil, $16.6 \mathrm{mg}, 95 \%$ yield, $81 \%$ ee, $10 / 1$ $\mathrm{dr},[\alpha]^{25} \mathrm{D}=+3.2\left(c=1.0, \mathrm{CHCl}_{3}\right) .{ }^{1} \mathrm{H}$ NMR $\left(400 \mathrm{MHz}, \mathrm{CDCl}_{3}\right) \delta 7.35-7.28(\mathrm{~m}, 5 \mathrm{H}), 7.22(\mathrm{t}$, $J=7.9 \mathrm{~Hz}, 1 \mathrm{H}), 6.78(\mathrm{~m}, 2 \mathrm{H}), 6.74-6.69(\mathrm{~m}, 1 \mathrm{H}), 4.78(\mathrm{~d}, J=14.5 \mathrm{~Hz}, 1 \mathrm{H}), 4.50(\mathrm{~d}, J=14.5$ $\mathrm{Hz}, 1 \mathrm{H}), 3.78(\mathrm{~s}, 3 \mathrm{H}), 3.67(\mathrm{~d}, J=30.6 \mathrm{~Hz}, 1 \mathrm{H}), 3.65(\mathrm{~s}, 3 \mathrm{H}), 3.46-3.35(\mathrm{~m}, 2 \mathrm{H}), 3.32-3.25$ (m, 1H), $2.18-1.92(\mathrm{~m}, 2 \mathrm{H}) .{ }^{13} \mathrm{C}\left\{{ }^{1} \mathrm{H}\right\} \mathrm{NMR}\left(151 \mathrm{MHz}, \mathrm{CDCl}_{3}\right) \delta 170.6,165.8,159.8,142.9$, $136.5,129.8,128.7,128.2,127.6,118.9,112.7,112.4,56.3,55.1,52.3,50.3,46.2,42.4,29.2$. HRMS (ESI)m/z: [M+H] $]^{+}$Calcd for $\mathrm{C}_{21} \mathrm{H}_{24} \mathrm{NO}_{4} \quad$ 354.1700; found 354.1702. The enantiomeric 
excess was determined by HPLC on Daicel Chiralcel OJ-3 Column (hexane : isopropanol $=80$ : 20, flow rate $=1 \mathrm{~mL} / \mathrm{min}, \mathrm{T}=25{ }^{\circ} \mathrm{C}$, $\mathrm{UV}$ detection at $\left.\lambda=210 \mathrm{~nm}\right), \mathrm{t}_{\mathrm{R} 1}=20.31 \mathrm{~min}(\operatorname{minor}), \mathrm{t}_{\mathrm{R} 2}$ $=27.45 \mathrm{~min}$ (major).

\section{Methyl (3R,4S)-1-benzyl-4-(4-fluorophenyl)-2-oxopiperidine-3-carboxylate (2i)}<smiles>COC(=O)[C@H]1C(=O)N(Cc2ccccc2)CC[C@H]1c1ccc(F)cc1</smiles>

Chemical Formula: $\mathrm{C}_{20} \mathrm{H}_{20} \mathrm{FNO}_{3}$

Exact Mass: 341.1427

$0.05 \mathrm{mmol}$ scale, yellow oil, $16.1 \mathrm{mg}, 96 \%$ yield, $90 \%$ ee,

$12 / 1 \mathrm{dr},[\alpha]^{25} \mathrm{D}=+4.7\left(c=1.0, \mathrm{CHCl}_{3}\right) .{ }^{1} \mathrm{H} \mathrm{NMR}\left(400 \mathrm{MHz}, \mathrm{CDCl}_{3}\right) \delta 7.40-7.24(\mathrm{~m}, 5 \mathrm{H}), 7.15$ $(\mathrm{dd}, J=8.0,5.5 \mathrm{~Hz}, 2 \mathrm{H}), 6.99(\mathrm{t}, J=8.5 \mathrm{~Hz}, 2 \mathrm{H}), 4.80(\mathrm{~d}, J=14.5 \mathrm{~Hz}, 1 \mathrm{H}), 4.48(\mathrm{~d}, J=14.5$ Hz, 1H), 3.64 (s, 3H), 3.59 (d, $J=11.0 \mathrm{~Hz}, 1 \mathrm{H}), 3.47-3.37$ (m, 2H), $3.31-3.26(\mathrm{~m}, 1 \mathrm{H}), 2.13$ $-1.87(\mathrm{~m}, 2 \mathrm{H}) .{ }^{19} \mathrm{~F}$ NMR $\left(376 \mathrm{MHz}, \mathrm{CDCl}_{3}\right) \delta-115.03 .{ }^{13} \mathrm{C}\left\{{ }^{1} \mathrm{H}\right\} \mathrm{NMR}\left(101 \mathrm{MHz}, \mathrm{CDCl}_{3}\right) \delta$ 170.4, 165.6, $161.9(\mathrm{~d}, J=245.8 \mathrm{~Hz}), 137.1(\mathrm{~d}, J=3.2 \mathrm{~Hz}), 136.5,128.7,128.3(\mathrm{~d}, J=8.1 \mathrm{~Hz})$, 128.2, 127.6, 115.7 (d, $J=21.4 \mathrm{~Hz}), 56.6,52.3,50.3,46.1,41.7,29.3$. HRMS (ESI)m/z: $[\mathrm{M}+\mathrm{H}]^{+}$Calcd for $\mathrm{C}_{20} \mathrm{H}_{21} \mathrm{FNO}_{3} \quad 342.1500$; found 342.1497.

The enantiomeric excess was determined by HPLC on Daicel Chiralcel AS-3 Column (hexane : isopropanol $=80: 20$, flow rate $=1 \mathrm{~mL} / \mathrm{min}, \mathrm{T}=25^{\circ} \mathrm{C}$, UV detection at $\left.\lambda=210 \mathrm{~nm}\right), \mathrm{t}_{\mathrm{R} 1}=$ $17.63 \min$ (major), $\mathrm{t}_{\mathrm{R} 2}=24.19 \min$ (minor).

Methyl (3R,4S)-1-benzyl-4-(3-fluorophenyl)-2-oxopiperidine-3-carboxylate (2j)<smiles>COC(=O)[C@H]1C(=O)N(Cc2ccccc2)CC[C@H]1c1cccc(F)c1</smiles>

Chemical Formula: $\mathrm{C}_{20} \mathrm{H}_{20} \mathrm{FNO}_{3}$

Exact Mass: $341.1427 \quad 0.05 \mathrm{mmol}$ scale, yellow oil, $17.5 \mathrm{mg}, 97 \%$ yield, $90 \%$ ee,

$11 / 1 \mathrm{dr},[\alpha]^{25}{ }_{\mathrm{D}}=+3.4\left(c=1.0, \mathrm{CHCl}_{3}\right) .{ }^{1} \mathrm{H}$ NMR $\left(400 \mathrm{MHz}, \mathrm{CDCl}_{3}\right) \delta 7.34-7.23(\mathrm{~m}, 6 \mathrm{H}), 6.99$ $-6.85(\mathrm{~m}, 3 \mathrm{H}), 4.80(\mathrm{~d}, J=14.5 \mathrm{~Hz}, 1 \mathrm{H}), 4.49(\mathrm{~d}, J=14.5 \mathrm{~Hz}, 1 \mathrm{H}), 3.65(\mathrm{~s}, 3 \mathrm{H}), 3.61(\mathrm{~d}, J=$ 
$11.0 \mathrm{~Hz}, 1 \mathrm{H}), 3.49-3.37(\mathrm{~m}, 2 \mathrm{H}), 3.32-3.27(\mathrm{~m}, 1 \mathrm{H}), 2.10-1.93(\mathrm{~m}, 2 \mathrm{H}) .{ }^{19} \mathrm{~F}$ NMR $(565$ $\left.\mathrm{MHz}, \mathrm{CDCl}_{3}\right) \delta-112.24 .{ }^{13} \mathrm{C}\left\{{ }^{1} \mathrm{H}\right\} \operatorname{NMR}\left(151 \mathrm{MHz}, \mathrm{CDCl}_{3}\right) \delta 170.3,165.5,163.0(\mathrm{~d}, J=246.6$ Hz), 143.9 (d, $J=6.9 \mathrm{~Hz}), 136.4,130.4(\mathrm{~d}, J=8.3 \mathrm{~Hz}), 128.7,128.2,127.6,122.4$ (d, $J=2.8$ $\mathrm{Hz}), 114.3(\mathrm{~d}, J=21.0 \mathrm{~Hz}), 113.8(\mathrm{~d}, J=21.7 \mathrm{~Hz}), 56.2,52.4,50.3,46.0,42.1$ (d, $J=1.4 \mathrm{~Hz})$, 29.1. HRMS (ESI)m/z: $[\mathrm{M}+\mathrm{H}]^{+}$Calcd for $\mathrm{C}_{20} \mathrm{H}_{21} \mathrm{FNO}_{3}$ 342.1500; found 342.1503. The enantiomeric excess was determined by HPLC on Daicel Chiralcel AD-3 Column (hexane : isopropanol $=85: 15$, flow rate $=1 \mathrm{~mL} / \mathrm{min}, \mathrm{T}=25^{\circ} \mathrm{C}, \mathrm{UV}$ detection at $\left.\lambda=210 \mathrm{~nm}\right), \mathrm{t}_{\mathrm{R} 1}=$ $14.74 \min$ (minor), $\mathrm{t}_{\mathrm{R} 2}=19.42 \min$ (major).

\section{Methyl (3R,4S)-1-benzyl-4-(3-bromophenyl)-2-oxopiperidine-3-carboxylate (2k)}<smiles>COC(=O)[C@H]1C(=O)N(Cc2ccccc2)CC[C@H]1c1cccc(Br)c1</smiles>

Chemical Formula: $\mathrm{C}_{20} \mathrm{H}_{20} \mathrm{BrNO}_{3}$ Exact Mass: 401.0627

$0.05 \mathrm{mmol}$ scale, yellow oil, $18.6 \mathrm{mg}, 93 \%$ yield, $85 \%$ ee, $11 / 1 \mathrm{dr},[\alpha]^{25} \mathrm{D}=+4.6\left(c=1.0, \mathrm{CHCl}_{3}\right) .{ }^{1} \mathrm{H} \mathrm{NMR}\left(400 \mathrm{MHz}, \mathrm{CDCl}_{3}\right) \delta 7.38-7.28(\mathrm{~m}, 7 \mathrm{H}), 7.18$ (t, $J=7.8 \mathrm{~Hz}, 1 \mathrm{H}), 7.11(\mathrm{~d}, J=7.8 \mathrm{~Hz}, 1 \mathrm{H}), 4.82(\mathrm{~d}, J=14.5 \mathrm{~Hz}, 1 \mathrm{H}), 4.46$ (d, $J=14.5 \mathrm{~Hz}$, 1H), 3.66 (s, 3H), 3.60 (d, $J=10.9 \mathrm{~Hz}, 1 \mathrm{H}), 3.46-3.36$ (m, 2H), 3.29 (m, 1H), $2.16-1.85$ (m, 2H). ${ }^{13} \mathrm{C}\left\{{ }^{1} \mathrm{H}\right\} \mathrm{NMR}\left(101 \mathrm{MHz}, \mathrm{CDCl}_{3}\right) \delta 170.3,165.4,143.6,136.4,130.5,130.4,130.0,128.7$, 128.2, 127.6, 125.4, 122.8, 56.1, 52.4, 50.3, 46.0, 42.0, 29.0. HRMS (ESI)m/z: $[\mathrm{M}+\mathrm{H}]^{+} \mathrm{Calcd}$ for $\mathrm{C}_{20} \mathrm{H}_{21} \mathrm{BrNO}_{3}$ 402.0699; found 402.0705.

The enantiomeric excess was determined by HPLC on Daicel Chiralcel AS-3 Column (hexane : isopropanol $=80: 20$, flow rate $=1 \mathrm{~mL} / \mathrm{min}, \mathrm{T}=25^{\circ} \mathrm{C}$, $\mathrm{UV}$ detection at $\left.\lambda=210 \mathrm{~nm}\right), \mathrm{t}_{\mathrm{R} 1}=$ $15.78 \mathrm{~min}$ (minor), $\mathrm{t}_{\mathrm{R} 2}=17.76 \mathrm{~min}$ (major). 
Methyl (3R,4S)-1-benzyl-2-oxo-4-(thiophen-2-yl)piperidine-3-carboxylate (2I)<smiles>COC(=O)[C@H]1C(=O)N(Cc2ccccc2)CC[C@H]1c1cccs1</smiles>

Chemical Formula: $\mathrm{C}_{18} \mathrm{H}_{19} \mathrm{NO}_{3} \mathrm{~S}$

Exact Mass: 329.1086

$0.05 \mathrm{mmol}$ scale, yellow oil, $15.1 \mathrm{mg}, 92 \%$ yield, $91 \%$ ee, $10 / 1 \mathrm{dr},[\alpha]^{25}=+3.9\left(c=1.0, \mathrm{CHCl}_{3}\right) .{ }^{1} \mathrm{H} \mathrm{NMR}\left(400 \mathrm{MHz}, \mathrm{CDCl}_{3}\right) \delta 7.36-7.27(\mathrm{~m}, 5 \mathrm{H}), 7.18$ $(\mathrm{d}, J=5.0 \mathrm{~Hz}, 1 \mathrm{H}), 6.92(\mathrm{dd}, J=5.0,3.6 \mathrm{~Hz}, 1 \mathrm{H}), 6.85(\mathrm{~d}, J=3.3 \mathrm{~Hz}, 1 \mathrm{H}), 4.76(\mathrm{~d}, J=14.6$ $\mathrm{Hz}, 1 \mathrm{H}), 4.50(\mathrm{~d}, J=14.6 \mathrm{~Hz}, 1 \mathrm{H}), 3.83-3.77(\mathrm{~m}, 1 \mathrm{H}), 3.73(\mathrm{~s}, 3 \mathrm{H}), 3.65(\mathrm{~d}, J=10.3 \mathrm{~Hz}$, $1 \mathrm{H}), 3.43-3.36(\mathrm{~m}, 1 \mathrm{H}), 3.32-3.26(\mathrm{~m}, 1 \mathrm{H}), 2.26-2.19(\mathrm{~m}, 1 \mathrm{H}), 2.07-1.97(\mathrm{~m}, 1 \mathrm{H}) .{ }^{13} \mathrm{C}\left\{{ }^{1} \mathrm{H}\right\}$ $\operatorname{NMR}\left(101 \mathrm{MHz}, \mathrm{CDCl}_{3}\right) \delta 170.4,165.1,144.8,136.4,128.7,128.2,127.6,126.9,124.1,123.9$, 57.2, 52.5, 50.3, 45.8, 37.8, 30.0. HRMS (ESI)m/z: $[\mathrm{M}+\mathrm{H}]^{+}$Calcd for $\mathrm{C}_{18} \mathrm{H}_{20} \mathrm{NO}_{3} \mathrm{~S}[\mathrm{M}+\mathrm{H}]^{+}$ 330.1158; found 330.1160.

The enantiomeric excess was determined by HPLC on Daicel Chiralcel AS-3 Column (hexane : isopropanol $=80: 20$, flow rate $=1 \mathrm{~mL} / \mathrm{min}, \mathrm{T}=25^{\circ} \mathrm{C}, \mathrm{UV}$ detection at $\left.\lambda=210 \mathrm{~nm}\right), \mathrm{t}_{\mathrm{R} 1}=$ 16.44 min (major), $\mathrm{t}_{\mathrm{R} 2}=20.31 \mathrm{~min}$ (minor).

\section{Methyl (3R,4S)-1-(4-methoxyphenyl)-4-methyl-2-oxopiperidine-3-carboxylate (2m)}<smiles>COC(=O)[C@@H]1C(=O)N(c2ccc(OC)cc2)CC[C@@H]1C</smiles>

Chemical Formula: $\mathrm{C}_{15} \mathrm{H}_{19} \mathrm{NO}_{4}$

Exact Mass: 277.1314

0.05 mmol scale, yellow solid (melting point: $86-91{ }^{\circ} \mathrm{C}$ ), 13.1 mg, $95 \%$ yield, $86 \%$ ee, $5 / 1 \mathrm{dr},[\alpha]^{25} \mathrm{D}=+4.0\left(c=1.0, \mathrm{CHCl}_{3}\right) .{ }^{1} \mathrm{H} \mathrm{NMR}\left(400 \mathrm{MHz}, \mathrm{CDCl}_{3}\right) \delta$ $7.21-7.08(\mathrm{~m}, 2 \mathrm{H}), 6.98-6.79(\mathrm{~m}, 2 \mathrm{H}), 3.80(\mathrm{~s}, 3 \mathrm{H}), 3.77(\mathrm{~s}, 3 \mathrm{H}), 3.64-3.52(\mathrm{~m}, 1 \mathrm{H}), 3.19$ $(\mathrm{d}, J=10.3 \mathrm{~Hz}, 1 \mathrm{H}), 2.53-2.38(\mathrm{~m}, 1 \mathrm{H}), 2.10-1.97(\mathrm{~m}, 1 \mathrm{H}), 1.80-1.60(\mathrm{~m}, 2 \mathrm{H}), 1.11(\mathrm{~d}, J$ $=6.6 \mathrm{~Hz}, 3 \mathrm{H}) .{ }^{13} \mathrm{C}\left\{{ }^{1} \mathrm{H}\right\} \mathrm{NMR}\left(101 \mathrm{MHz}, \mathrm{CDCl}_{3}\right) \delta 171.0,166.0,158.2,135.4,127.2,114.4$, 57.7, 55. 5, 52.3, 50.5, 31.7, 30.0, 20.1. HRMS (ESI)m/z: $[\mathrm{M}+\mathrm{H}]^{+}$Calcd for $\mathrm{C}_{15} \mathrm{H}_{20} \mathrm{NO}_{4}$ 278.1387; found 278.1389.

The enantiomeric excess was determined by HPLC on Daicel Chiralcel OD-3 Column (hexane : 
isopropanol $=87.5: 12.5$, flow rate $=1 \mathrm{~mL} / \mathrm{min}, \mathrm{T}=25^{\circ} \mathrm{C}, \mathrm{UV}$ detection at $\left.\lambda=210 \mathrm{~nm}\right), \mathrm{t}_{\mathrm{R} 1}=$ $30.88 \min$ (minor), $\mathrm{t}_{\mathrm{R} 2}=37.60 \min$ (major).

\section{Methyl (3R,4S)-1-benzyl-4-cyclohexyl-2-oxopiperidine-3-carboxylate (2n)}<smiles>COC(=O)[C@H]1C(=O)N(Cc2ccccc2)CC[C@H]1C1CCCCC1</smiles>

\section{Chemical Formula:}

$$
\mathrm{C}_{20} \mathrm{H}_{27} \mathrm{NO}_{3}
$$

Exact Mass: $329.19910 .05 \mathrm{mmol}$ scale, yellow solid (melting point: $76-79{ }^{\circ} \mathrm{C}$ ), $14.8 \mathrm{mg}, 90 \%$ yield, $91 \%$ ee, $7 / 1 \mathrm{dr},[\alpha]^{25}=+7.0\left(c=1.0, \mathrm{CHCl}_{3}\right) .{ }^{1} \mathrm{H} \mathrm{NMR}\left(400 \mathrm{MHz}, \mathrm{CDCl}_{3}\right) \delta 7.35-7.25$ (m, 5H), $4.68(\mathrm{~d}, J=14.6 \mathrm{~Hz}, 1 \mathrm{H}), 4.51(\mathrm{~d}, J=14.6 \mathrm{~Hz}, 1 \mathrm{H}), 3.79(\mathrm{~s}, 3 \mathrm{H}), 3.44(\mathrm{~d}, J=9.9 \mathrm{~Hz}$, $1 \mathrm{H}), 3.35-3.11(\mathrm{~m}, 2 \mathrm{H}), 2.18-2.06(\mathrm{~m}, 1 \mathrm{H}), 1.89-1.80(\mathrm{~m}, 1 \mathrm{H}), 1.72-1.56(\mathrm{~m}, 4 \mathrm{H}), 1.56$ $-1.43(\mathrm{~m}, 1 \mathrm{H}), 1.33-1.03(\mathrm{~m}, 6 \mathrm{H}), 0.90(\mathrm{~m}, 1 \mathrm{H}) .{ }^{13} \mathrm{C}\left\{{ }^{1} \mathrm{H}\right\} \mathrm{NMR}\left(101 \mathrm{MHz}, \mathrm{CDCl}_{3}\right) \delta 171.8$, $166.5,136.7,128.6,128.0,127.4,53.0,52.4,50.2,45.9,41.6,39.8,30.8,27.7,26.5,26.4,23.2$. HRMS (ESI)m/z: $[\mathrm{M}+\mathrm{H}]^{+}$Calcd for $\mathrm{C}_{20} \mathrm{H}_{28} \mathrm{NO}_{3} 330.2064$; found 330.2069.

The enantiomeric excess was determined by HPLC on Daicel Chiralcel AS-3 Column (hexane : isopropanol $=80: 20$, flow rate $=1 \mathrm{~mL} / \mathrm{min}, \mathrm{T}=25^{\circ} \mathrm{C}, \mathrm{UV}$ detection at $\left.\lambda=210 \mathrm{~nm}\right), \mathrm{t}_{\mathrm{R} 1}=7.91$ $\min \left(\right.$ major), $\mathrm{t}_{\mathrm{R} 2}=10.49 \min$ (minor).

\section{Methyl (3R,4S)-1-methyl-2-oxo-4-phenylpiperidine-3-carboxylate (2o)}<smiles>COC(=O)[C@H]1C(=O)N(C)CC[C@@H]1c1ccccc1</smiles>

Chemical Formula: $\mathrm{C}_{14} \mathrm{H}_{17} \mathrm{NO}_{3}$

Exact Mass: 247.1208

$0.05 \mathrm{mmol}$ scale, yellow oil, $10.6 \mathrm{mg}, 86 \%$ yield, $84 \%$ ee, $15 / 1$ $\mathrm{dr},[\alpha]^{25} \mathrm{D}=+3.1\left(c=1.0, \mathrm{CHCl}_{3}\right) .{ }^{1} \mathrm{H}$ NMR $\left(600 \mathrm{MHz}, \mathrm{CDCl}_{3}\right) \delta 7.32(\mathrm{t}, J=7.6 \mathrm{~Hz}, 3 \mathrm{H}), 7.19$ (d, $J=7.6 \mathrm{~Hz}, 2 \mathrm{H}), 3.60(\mathrm{~s}, 3 \mathrm{H}), 3.57(\mathrm{~d}, J=11.0 \mathrm{~Hz}, 1 \mathrm{H}), 3.52(\mathrm{~m}, 1 \mathrm{H}), 3.44(\mathrm{~m}, 1 \mathrm{H}), 3.34$ (m, 1H), $3.01(\mathrm{~s}, 3 \mathrm{H}), 2.10(\mathrm{~m}, 2 \mathrm{H}) .{ }^{13} \mathrm{C}\left\{{ }^{1} \mathrm{H}\right\} \mathrm{NMR}\left(151 \mathrm{MHz}, \mathrm{CDCl}_{3}\right) \delta 170.55,165.73,141.34$, $128.84,127.32,126.73,56.24,52.23,48.92,42.51,34.83,29.29$.

The enantiomeric excess was determined by HPLC on Daicel Chiralcel AD-3 Column (hexane : 
isopropanol $=85: 15$, flow rate $=1 \mathrm{~mL} / \mathrm{min}, \mathrm{T}=25^{\circ} \mathrm{C}, \mathrm{UV}$ detection at $\left.\lambda=220 \mathrm{~nm}\right), \mathrm{t}_{\mathrm{R} 1}=$ $11.34 \min$ (minor), $\mathrm{t}_{\mathrm{R} 2}=12.55 \min$ (major).

\section{One-pot asymmetric transformations of 1 a to 3}

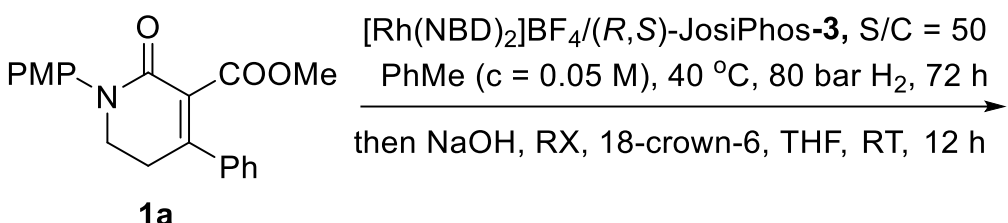

$\mathrm{RX}: \mathrm{Me}-\mathrm{I} / \mathrm{Ph} \widehat{\mathrm{Br}}^{\mathrm{I}}$<smiles>[R]C(=O)C1(C(C)=O)C(=O)N(P=[W])CC[C@H]1c1ccccc1</smiles>

3a-3c

Following the general procedure for asymmetric hydrogenation of $1 \mathrm{a}(0.05 \mathrm{mmol})$, the reaction solution was evaporated under reduced pressure. The residue was then dissolved in THF $(1.0 \mathrm{~mL})$, followed by addition of sodium hydroxide $(8.0 \mathrm{mg}, 0.20$ mmol), electrophile RX (0.20 mmol) and 18-crown-6 (3.93 mg, $0.015 \mathrm{mmol})$. The resulting mixture was stirred at RT for $12 \mathrm{~h}$. The reaction mixture was concentrated in vacuo and then purified by column chromatography on silica gel using hexanes and ethyl acetate to give the desirable products 3 . The enantiomeric excess was determined by chiral HPLC.

Methyl (3R,4R)-1-(4-methoxyphenyl)-3-methyl-2-oxo-4-phenylpiperidine-3-carboxylate (3a)<smiles>COc1ccc(N2CC[C@H](c3ccccc3)[C@](C)(C(C)=O)C2=O)cc1</smiles>

Chemical Formula: $\mathrm{C}_{21} \mathrm{H}_{23} \mathrm{NO}_{4}$

Exact Mass: $353.1627 \quad 0.05 \mathrm{mmol}$ scale, yellow oil, $15.2 \mathrm{mg}, 86 \%$ yield, $91 \%$ ee, $>20 / 1 \mathrm{dr},[\alpha]^{25}=-74.4\left(c=1.0, \mathrm{CHCl}_{3}\right) .{ }^{1} \mathrm{H} \mathrm{NMR}\left(600 \mathrm{MHz}, \mathrm{CDCl}_{3}\right) \delta 7.32(\mathrm{t}, J=7.2 \mathrm{~Hz}$, 2H), $7.29-7.27$ (m, 1H), $7.26-7.21(\mathrm{~m}, 2 \mathrm{H}), 7.19-7.14(\mathrm{~m}, 2 \mathrm{H}), 6.96-6.89(\mathrm{~m}, 2 \mathrm{H}), 3.91$ $-3.86(\mathrm{~m}, 1 \mathrm{H}), 3.81(\mathrm{~s}, 3 \mathrm{H}), 3.76-3.72(\mathrm{~m}, 1 \mathrm{H}), 3.56(\mathrm{~s}, 3 \mathrm{H}), 3.11(\mathrm{dd}, J=13.5,2.2 \mathrm{~Hz}, 1 \mathrm{H})$, $3.07-3.00(\mathrm{~m}, 1 \mathrm{H}), 1.97-1.94(\mathrm{~m}, 1 \mathrm{H}), 1.56(\mathrm{~s}, 3 \mathrm{H}) .{ }^{13} \mathrm{C}\left\{{ }^{1} \mathrm{H}\right\} \mathrm{NMR}\left(151 \mathrm{MHz}, \mathrm{CDCl}_{3}\right) \delta 171.6$, $169.9,158.2,139.8,136.2,128.3,128.1,127.6,127.2,114.4,56.4,55.5,52.0,51.3,50.1,25.6$, 21.9. HRMS (ESI)m/z: $[\mathrm{M}+\mathrm{H}]^{+}$Calcd for $\mathrm{C}_{21} \mathrm{H}_{24} \mathrm{NO}_{4}$ 354.1700; found 354.1702. 
The enantiomeric excess was determined by HPLC on Daicel Chiralcel AD-3 Column (hexane : isopropanol $=80: 20$, flow rate $=1 \mathrm{~mL} / \mathrm{min}, \mathrm{T}=25^{\circ} \mathrm{C}, \mathrm{UV}$ detection at $\left.\lambda=210 \mathrm{~nm}\right), \mathrm{t}_{\mathrm{R} 1}=9.23$ $\min$ (major), $\mathrm{t}_{\mathrm{R} 2}=23.23 \min$ (minor).

Methyl (3R,4R)-3-allyl-1-(4-methoxyphenyl)-2-oxo-4-phenylpiperidine-3-carboxylate (3b)<smiles>C=CC[C@]1(C(=O)OC)C(=O)N(c2ccc(OC)cc2)CCC1c1ccccc1</smiles>

Chemical Formula: $\mathrm{C}_{23} \mathrm{H}_{25} \mathrm{NO}_{4}$

Exact Mass: $379.1784 \quad 0.05 \mathrm{mmol}$ scale, yellow oil, $15.5 \mathrm{mg}, 82 \%$ yield, $90 \%$ ee, $>20 / 1 \mathrm{dr},[\alpha]^{25} \mathrm{D}=-91.9\left(c=1.0, \mathrm{CHCl}_{3}\right) .{ }^{1} \mathrm{H} \mathrm{NMR}\left(600 \mathrm{MHz}, \mathrm{CDCl}_{3}\right) \delta 7.26(\mathrm{t}, J=7.4 \mathrm{~Hz}$, 2H), $7.21(\mathrm{~d}, J=7.2 \mathrm{~Hz}, 1 \mathrm{H}), 7.16(\mathrm{~d}, J=8.8 \mathrm{~Hz}, 2 \mathrm{H}), 7.12(\mathrm{~d}, J=7.3 \mathrm{~Hz}, 2 \mathrm{H}), 6.86(\mathrm{~d}, J=$ $8.8 \mathrm{~Hz}, 2 \mathrm{H}), 5.89-5.67$ (m, 1H), 5.29 (dd, $J=20.6,13.6 \mathrm{~Hz}, 2 \mathrm{H}), 3.79-3.73(\mathrm{~m}, 1 \mathrm{H}), 3.74$ (s, 3H), $3.65-3.60(\mathrm{~m}, 1 \mathrm{H}), 3.48$ (s, 3H), $3.36(\mathrm{dd}, J=13.5,2.7 \mathrm{~Hz}, 1 \mathrm{H}), 3.04-2.92(\mathrm{~m}, 2 \mathrm{H})$, $2.50(\mathrm{dd}, J=14.2,9.8 \mathrm{~Hz}, 1 \mathrm{H}), 1.88-1.85(\mathrm{~m}, 1 \mathrm{H}) \cdot{ }^{13} \mathrm{C}\left\{{ }^{1} \mathrm{H}\right\} \mathrm{NMR}\left(101 \mathrm{MHz}, \mathrm{CDCl}_{3}\right) \delta 171.3$, $168.7,158.3,139.9,136.1,133.7,128.4,128.0,127.5,127.3,120.3,114.5,60.1,55.5,52.16$, 51.3, 44.1, 37.4, 25.7. HRMS (ESI)m/z: $[\mathrm{M}+\mathrm{H}]^{+}$Calcd for $\mathrm{C}_{23} \mathrm{H}_{26} \mathrm{NO}_{4}$ 380.1856; found 380.1860.

The enantiomeric excess was determined by HPLC on Daicel Chiralcel AD-3 Column (hexane : isopropanol $=80: 20$, flow rate $=1 \mathrm{~mL} / \mathrm{min}, \mathrm{T}=25^{\circ} \mathrm{C}, \mathrm{UV}$ detection at $\left.\lambda=210 \mathrm{~nm}\right), \mathrm{t}_{\mathrm{R} 1}=$ $10.31 \min$ (major), $\mathrm{t}_{\mathrm{R} 2}=15.52 \mathrm{~min}$ (minor).

\section{Methyl (3R,4R)-3-benzyl-1-(4-methoxyphenyl)-2-oxo-4-phenylpiperidine-3-carboxylate} (3c)<smiles>COc1ccc(N2CCC([C@H](Cc3ccccc3)c3ccccc3)[C](Cc3ccccc3)C2=O)cc1</smiles>

Chemical Formula: $\mathrm{C}_{27} \mathrm{H}_{27} \mathrm{NO}_{4}$

Exact Mass: $429.1940 \quad 0.05 \mathrm{mmol}$ scale, yellow oil, $18.0 \mathrm{mg}, 84 \%$ yield, $89 \%$ ee, $>20 / 1 \mathrm{dr},[\alpha]^{25} \mathrm{D}=-64.8\left(c=1.0, \mathrm{CHCl}_{3}\right) .{ }^{1} \mathrm{H} \mathrm{NMR}\left(600 \mathrm{MHz}, \mathrm{CDCl}_{3}\right) \delta 7.36-7.20(\mathrm{~m}$, 
$10 \mathrm{H}), 7.02(\mathrm{~d}, J=8.8 \mathrm{~Hz}, 2 \mathrm{H}), 6.85(\mathrm{~d}, J=8.8 \mathrm{~Hz}, 2 \mathrm{H}), 3.75$ (s, 3H), 3.64 (d, $J=14.0 \mathrm{~Hz}, 1 \mathrm{H})$, $3.50(\mathrm{~s}, 3 \mathrm{H}), 3.39-3.33(\mathrm{~m}, 1 \mathrm{H}), 3.29(\mathrm{dd}, J=13.4,3.0 \mathrm{~Hz}, 1 \mathrm{H}), 3.19(\mathrm{~m}, 1 \mathrm{H}), 3.05(\mathrm{~d}, J=$ $14.0 \mathrm{~Hz}, 1 \mathrm{H}), 2.89-2.76(\mathrm{~m}, 1 \mathrm{H}), 1.74-1.71(\mathrm{~m}, 1 \mathrm{H}) .{ }^{13} \mathrm{C}\left\{{ }^{1} \mathrm{H}\right\} \mathrm{NMR}\left(151 \mathrm{MHz}, \mathrm{CDCl}_{3}\right) \delta$ 171.5, 168.9, 158.2, 140.0, 136.8, 136.2, 131.3, 128.5, 128.4, 128.2, 127.6, 127.0, 126.9, 114.4, 61.8, 55.5, 52.1, 50.7, 43.7, 38.6, 26.6. HRMS (ESI)m/z: $[\mathrm{M}+\mathrm{H}]^{+}$Calcd for $\mathrm{C}_{27} \mathrm{H}_{28} \mathrm{NO}_{4}$ 430.2013; found 430.2017 .

The enantiomeric excess was determined by HPLC on Daicel Chiralcel AS-3 Column (hexane : isopropanol $=80: 20$, flow rate $=1 \mathrm{~mL} / \mathrm{min}, \mathrm{T}=25^{\circ} \mathrm{C}, \mathrm{UV}$ detection at $\left.\lambda=210 \mathrm{~nm}\right), \mathrm{t}_{\mathrm{R} 1}=7.88$ $\min$ (major), $\mathrm{t}_{\mathrm{R} 2}=15.85 \mathrm{~min}$ (minor). 


\section{Scale-up reactions and synthetic applications}

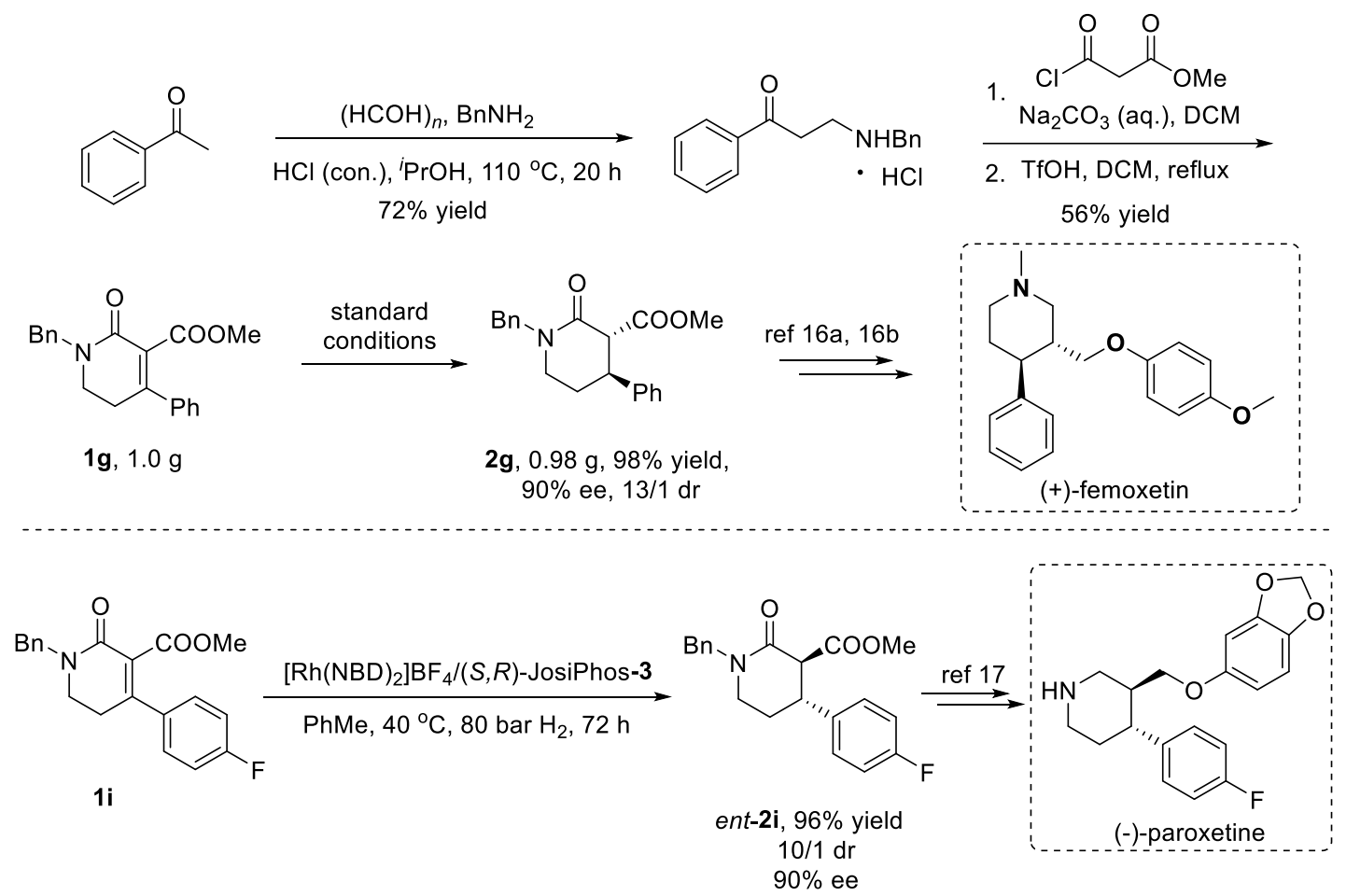

A mixture of acetophenone (1.0 g, $8 \mathrm{mmol}, 1$ equiv.), benzylamine ( $2.4 \mathrm{~g}, 10 \mathrm{mmol}, 1.2$ equiv.), paraformaldehyde ( $4.7 \mathrm{~g}, 12 \mathrm{mmol}, 1.5$ equiv.) and $\mathrm{HCl}(1 \mathrm{~mL}$, con., $0.125 \mathrm{~mL} / \mathrm{mmol})$ in ${ }^{i} \mathrm{PrOH}$ $(20 \mathrm{~mL})$ was stirred and heated in a sealed tube at $110{ }^{\circ} \mathrm{C}$ for $20 \mathrm{~h}$. After cooling to room temperature, ${ }^{i} \mathrm{PrOH}$ was removed under reduced pressure and EtOAc was added. The mixture was strongly stirred for $3 \mathrm{~h}$ at room temperature and then filtered. ${ }^{6}$ The solid was washed with acetone and the crude product was used in the next step without further purification. The next two steps are the same as that mentioned above for the synthesis of substrates.

To a $10 \mathrm{~mL}$ vial was added the catalyst precursor $\left[\mathrm{Rh}(\mathrm{NBD})_{2}\right] \mathrm{BF}_{4}\left(22.5 \mathrm{mg}, 3 \times 10^{-2} \mathrm{mmol}\right)$, $(R, S)$-JosiPhos-3 (46.8 mg, $\left.6.6 \times 10^{-2} \mathrm{mmol}\right)$ and anhydrous PhMe $(5.0 \mathrm{~mL})$ in an argon-filled glovebox. The mixture was stirred for $2.0 \mathrm{~h}$ at RT, giving orange solution. On the other hand, $1 \mathrm{~g}(3.1 \mathrm{mmol}, 1.0 \mathrm{~g})$ was added into a $50 \mathrm{~mL}$ hydrogenation vessel, followed by addition of $27.5 \mathrm{~mL}$ anhydrous PhMe and then a solution of preprepared catalyst $(\sim 2.6 \mathrm{~mL})$. Then the vessel was placed in an autoclave which was then charged with 80 bar of $\mathrm{H}_{2}$ and stirred at 40 ${ }^{\circ} \mathrm{C}$ for $72 \mathrm{~h}$. After slowly releasing the hydrogen pressure, the reaction mixture was passed through a short column of silica gel to remove the metal complex. The filtration was concentrated and purified by flash chromatography (neutral $\mathrm{Al}_{2} \mathrm{O}_{3}$ ) with ethyl acetate to afford the desired product $2 \mathrm{~g}$ as a pale yellow oil ( $0.98 \mathrm{~g}, 98 \%$ yield, $90 \%$ ee, $13 / 1 \mathrm{dr})$. 
Ent-2i was prepared by the above-mentioned method except using ligand (S,R)-JosiPhos-3.

Ent-2i, $\quad 0.05 \mathrm{mmol}$ scale, yellow oil, $16.0 \mathrm{mg}, 96 \%$ yield, $90 \%$ ee, $10 / 1 \mathrm{dr},[\alpha]^{25} \mathrm{D}=-5.1(c=$ 1.0, $\left.\mathrm{CHCl}_{3}\right)$. The enantiomeric excess was determined by HPLC on Daicel Chiralcel AS-3 Column (hexane : isopropanol $=80: 20$, flow rate $=1 \mathrm{~mL} / \mathrm{min}, \mathrm{T}=25^{\circ} \mathrm{C}$, UV detection at $\lambda=$ $210 \mathrm{~nm}$ ), $\mathrm{t}_{\mathrm{R} 1}=18.67 \min \left(\right.$ minor), $\mathrm{t}_{\mathrm{R} 2}=25.09 \min$ (major).

7. Monitoring the diastereomeric ratio of the product along with the reaction process<smiles>[R16]N(P)N1CCC(c2ccccc2)=C(C(=O)OC)C1=O</smiles>

$1 \mathrm{a}$<smiles>COC(=O)[C@H]1C(=O)N([PH](C)(C)C)CC[C@@H]1c1ccccc1</smiles>

2a

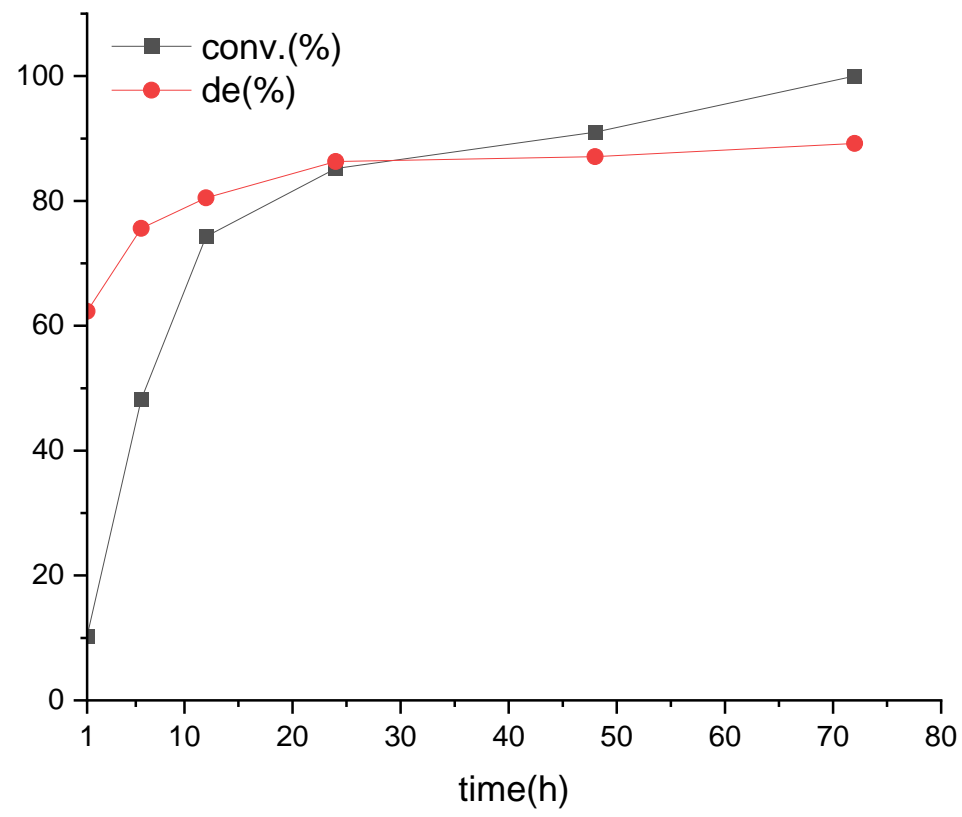

\begin{tabular}{|c|c|c|c|c|c|c|}
\hline Time (h) & 1 & 6 & 12 & 24 & 48 & 72 \\
\hline $\begin{array}{c}\mathrm{de} \\
\text { (trans/cis })\end{array}$ & 62.3 & 75.6 & 80.5 & 86.3 & 87.1 & 89.2 \\
\hline $\begin{array}{c}\mathrm{dr} \\
\text { (trans/cis) }\end{array}$ & $4.3 / 1$ & $7.2 / 1$ & $9.3 / 1$ & $13.6 / 1$ & $14.5 / 1$ & $17.5 / 1$ \\
\hline conv. & 10.3 & 48.2 & 74.3 & 85.2 & 91.0 & 100 \\
\hline
\end{tabular}

To a $20 \mathrm{~mL}$ vial was added the catalyst precursor $\left[\mathrm{Rh}(\mathrm{NBD})_{2}\right] \mathrm{BF}_{4}\left(22.5 \mathrm{mg}, 3 \times 10^{-2} \mathrm{mmol}\right)$, $(R, S)$-JosiPhos-3 $\left(46.8 \mathrm{mg}, 6.6 \times 10^{-2} \mathrm{mmol}\right)$ and anhydrous PhMe $(6 \mathrm{~mL})$ in an argon-filled 
glovebox. The mixture was stirred for $0.5 \mathrm{~h}$ at $25^{\circ} \mathrm{C}$ to give orange solution. On the other hand, to six hydrogenation vessels were added $\mathbf{1 a}(0.3 \mathrm{mmol}$ for each), followed by addition of $5 \mathrm{~mL}$ anhydrous PhMe for each. An aliquot of the catalyst solution $(\sim 600 \mu \mathrm{L})$ was added into each vessels via an injection port. Then the vessels were placed in six autoclaves which were then charged with 80 bar of $\mathrm{H}_{2}$ and stirred at $40{ }^{\circ} \mathrm{C}$ for indicated time. After indicated time, the hydrogen pressure was slowly released and the reaction solvent was evaporated. The residue was directly subjected to ${ }^{1} \mathrm{H}$ NMR experiments to determine the conv. and de values. The parallel reactions were repeated for twice. 


\section{NMR Spectra}

${ }^{1} \mathrm{H}$ NMR (400 MHz, $\left.\mathrm{CDCl}_{3}\right)$ of compound 1a

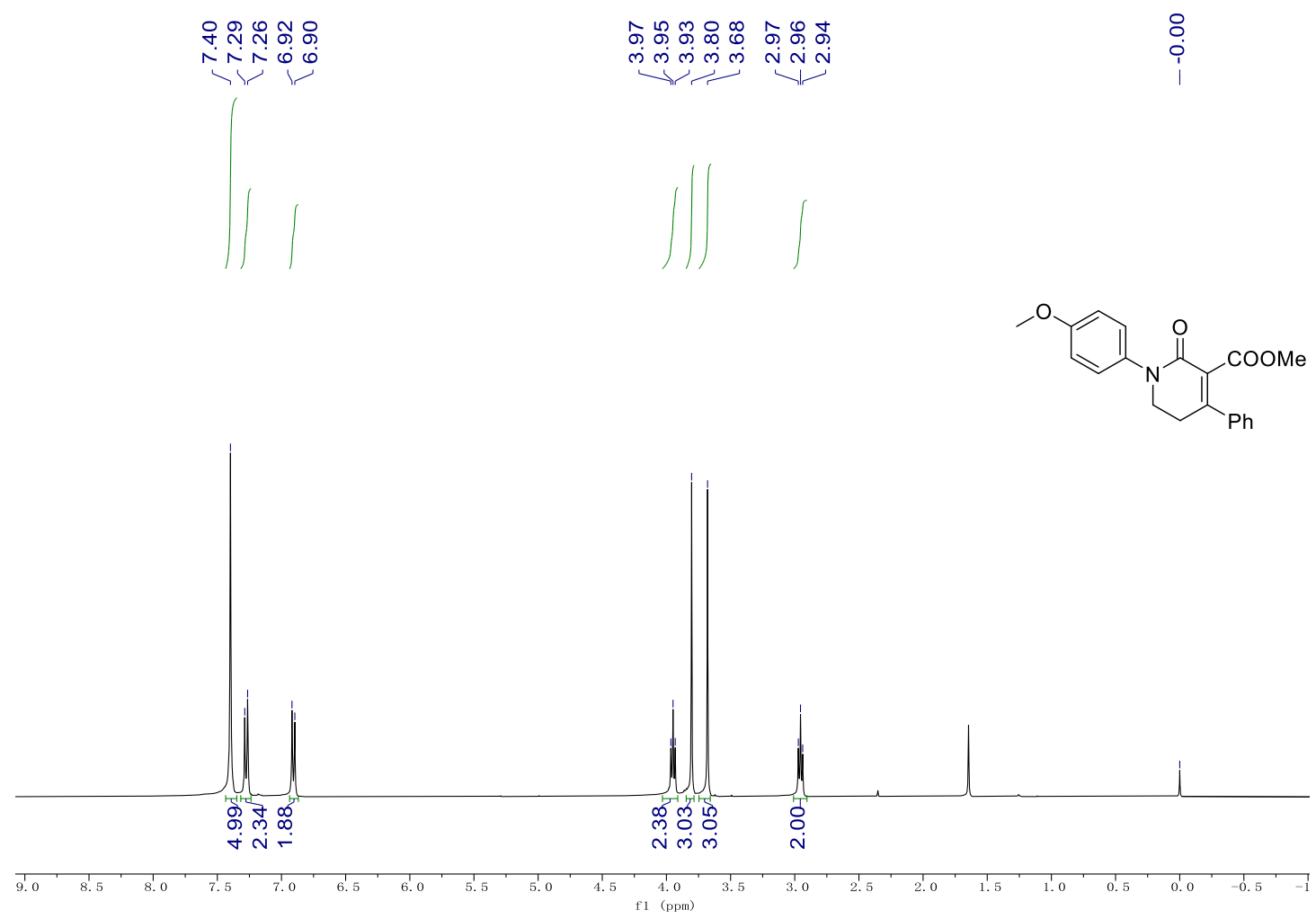

${ }^{13} \mathrm{C}\left\{{ }^{1} \mathrm{H}\right\}$ NMR (101 MHz, $\left.\mathrm{CDCl}_{3}\right)$ of compound 1a

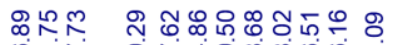

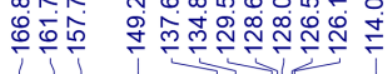

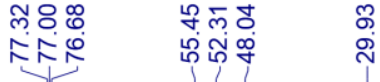

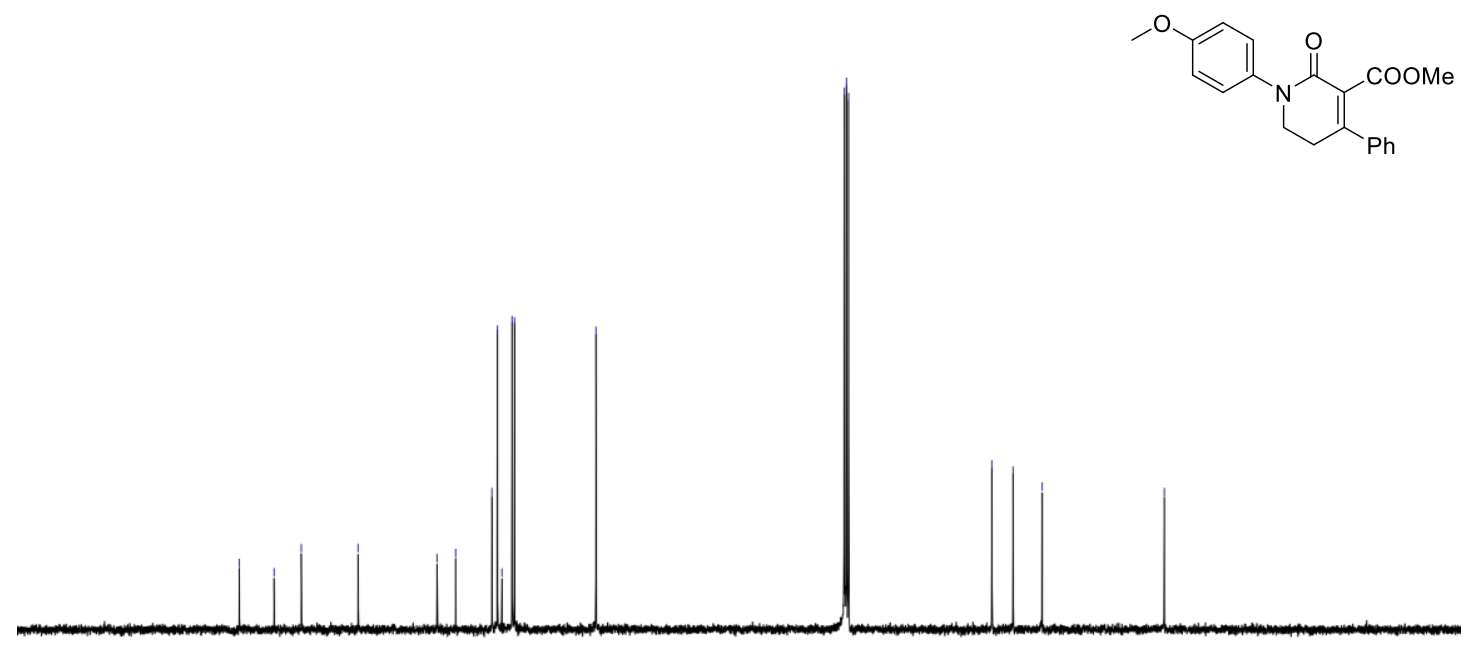


${ }^{1} \mathrm{H}$ NMR (400 MHz, $\mathrm{CDCl}_{3}$ ) of compound $1 \mathrm{~b}$

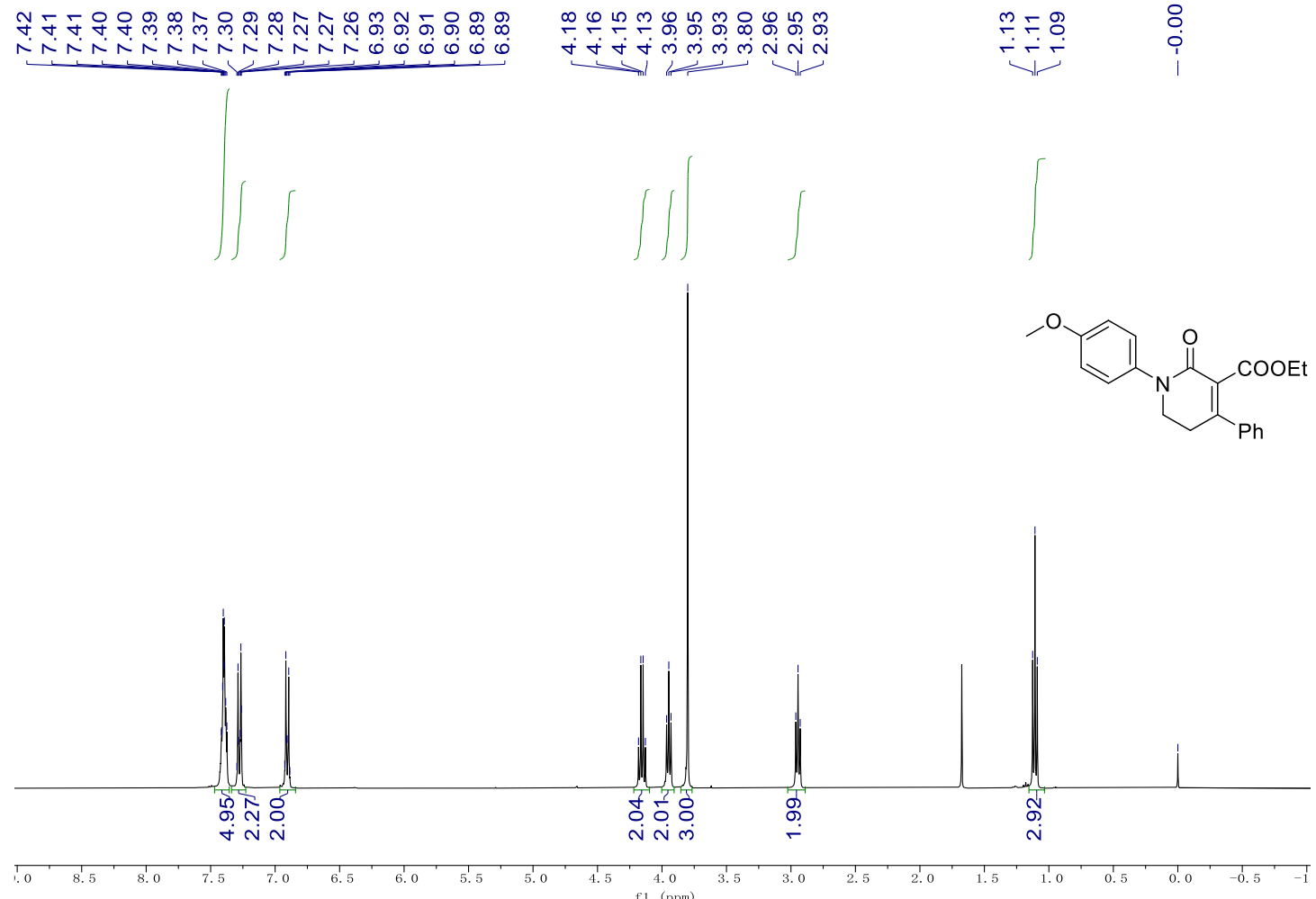

${ }^{13} \mathrm{C}\left\{{ }^{1} \mathrm{H}\right\}$ NMR (101 MHz, $\left.\mathrm{CDCl}_{3}\right)$ of compound 1b

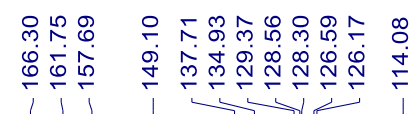

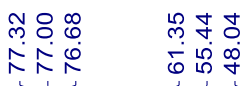

$\begin{array}{ll}\text { Nे } & \stackrel{9}{ก} \\ \stackrel{m}{1} & \stackrel{1}{1}\end{array}$

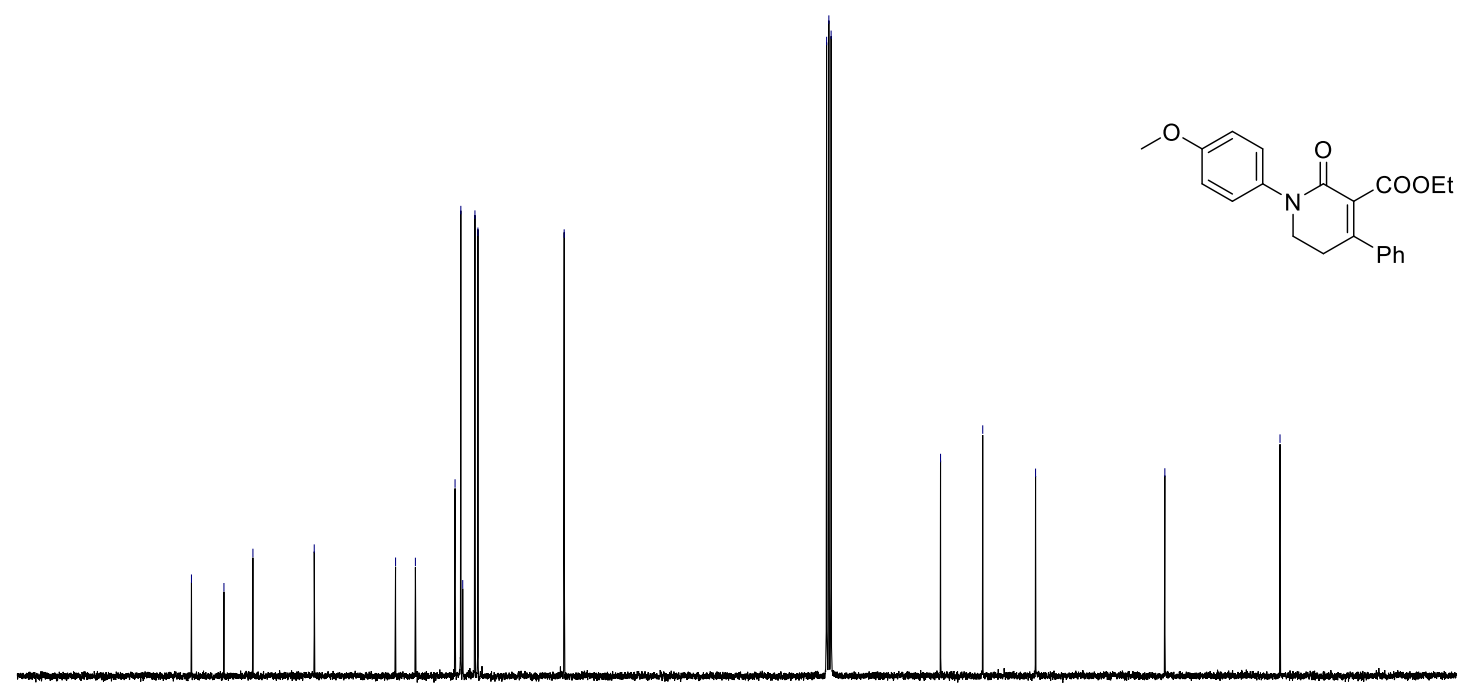

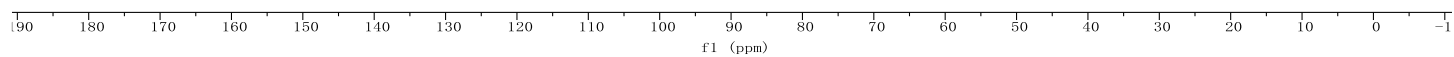


${ }^{1} \mathrm{H}$ NMR (400 MHz, $\left.\mathrm{CDCl}_{3}\right)$ of compound $1 \mathrm{c}$

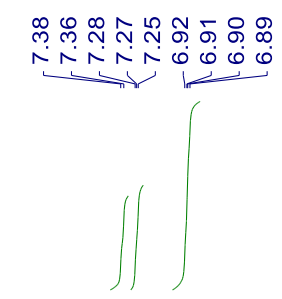

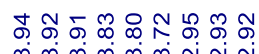

लंलूलm लं
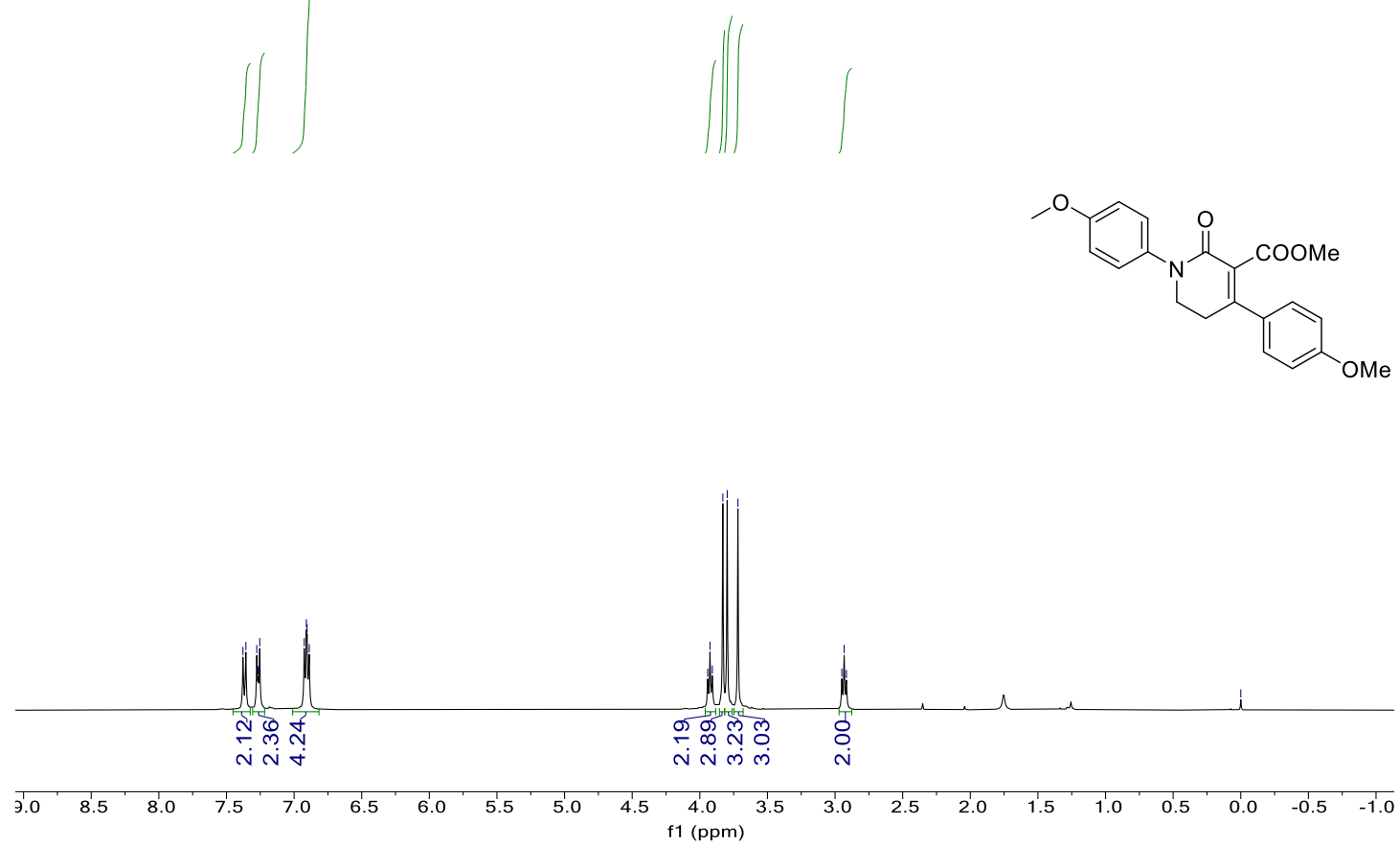

${ }^{13} \mathrm{C}\left\{{ }^{1} \mathrm{H}\right\}$ NMR (101 MHz, $\left.\mathrm{CDCl}_{3}\right)$ of compound 1c

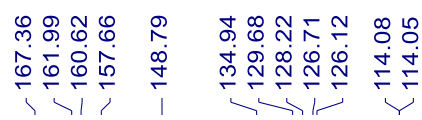

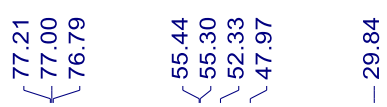

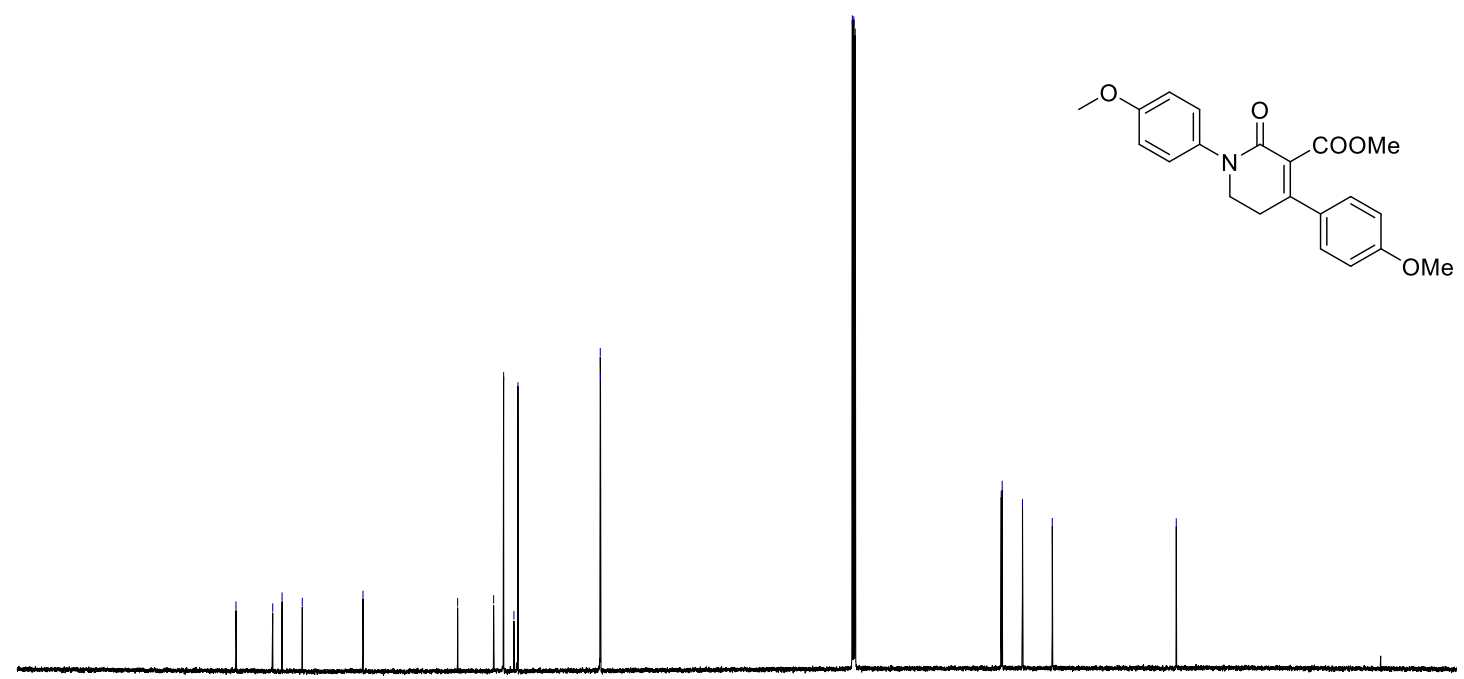

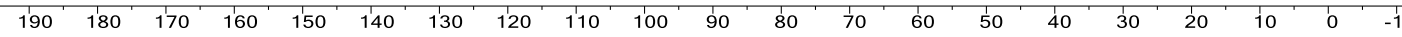


${ }^{1} \mathrm{H}$ NMR (400 MHz, $\mathrm{CDCl}_{3}$ ) of compound 1d

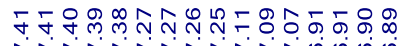

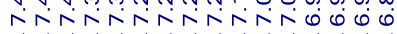

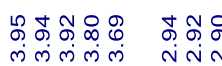

$\stackrel{\circ}{i}$

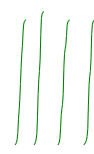
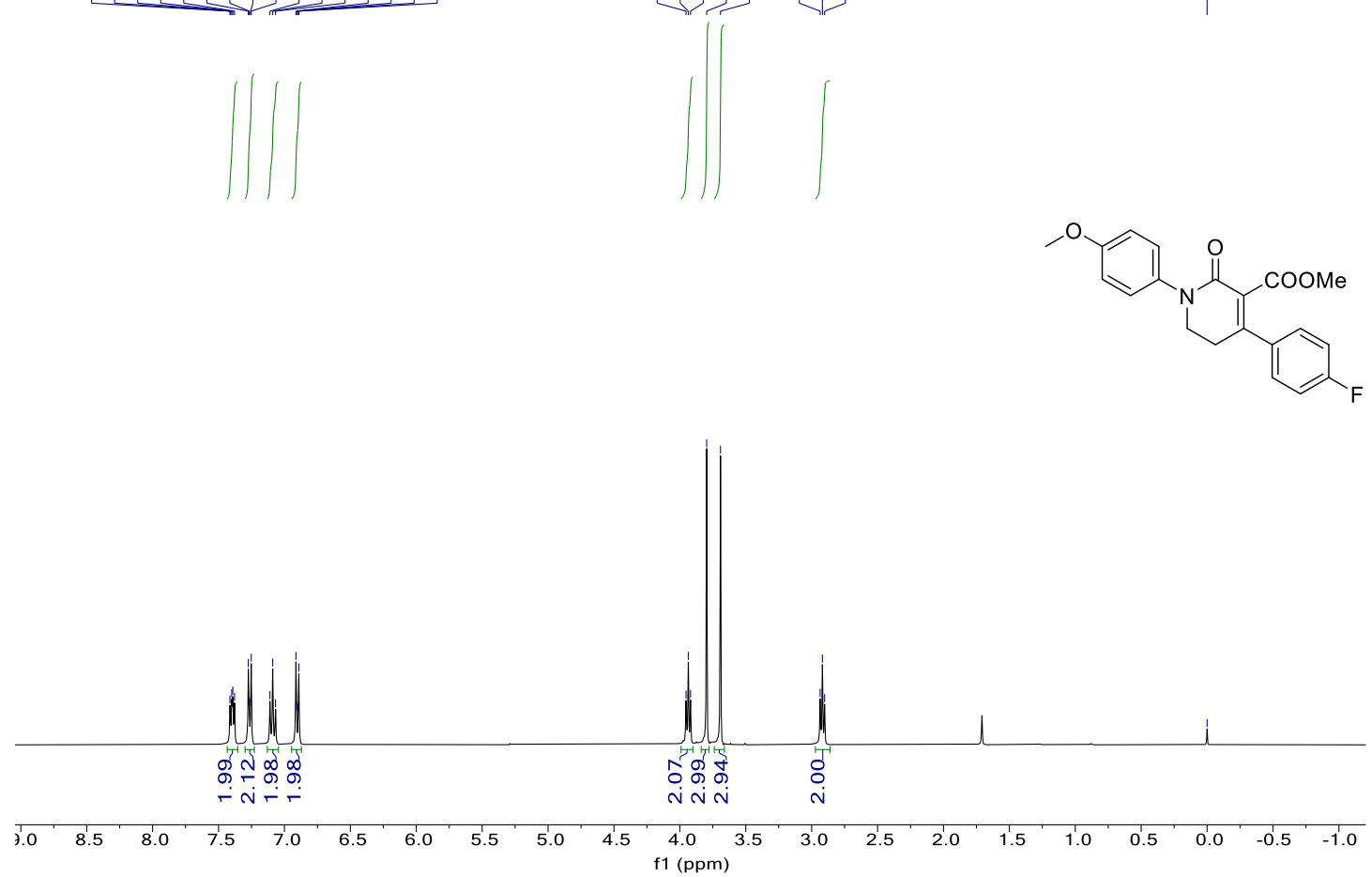

${ }^{19}$ F NMR (565 MHz, $\mathrm{CDCl}_{3}$ ) of compound 1d

$\stackrel{N}{N}$
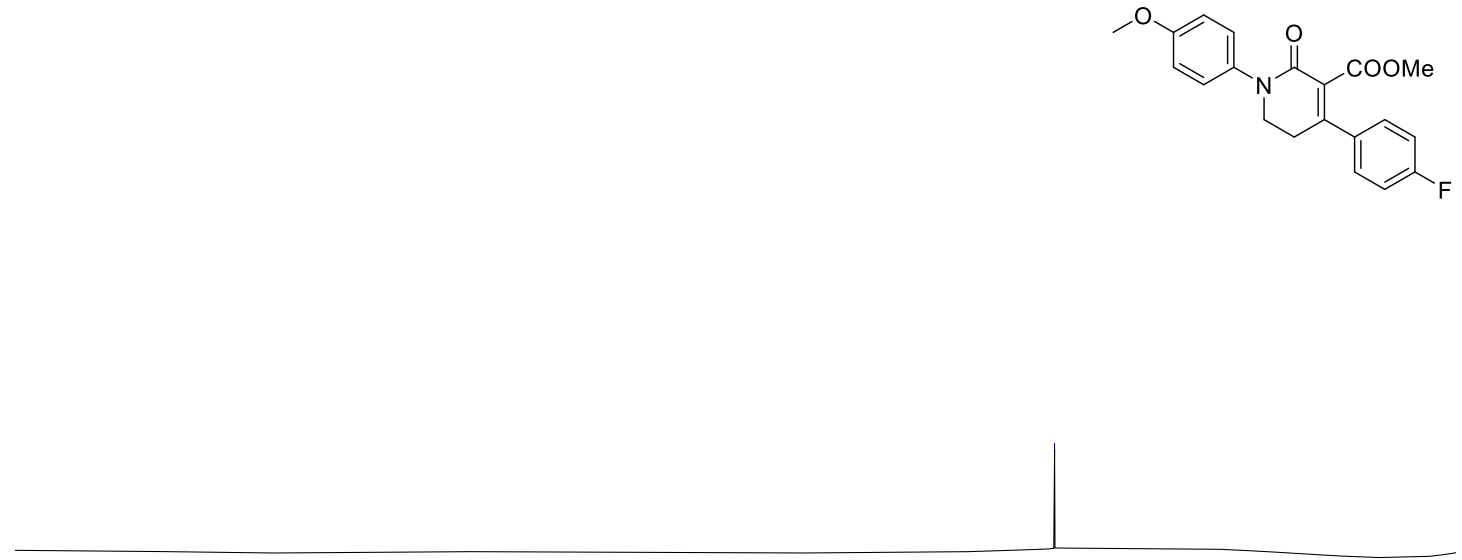
${ }^{13} \mathrm{C}\left\{{ }^{1} \mathrm{H}\right\}$ NMR (101 MHz, $\left.\mathrm{CDCl}_{3}\right)$ of compound 1d

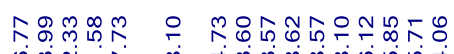

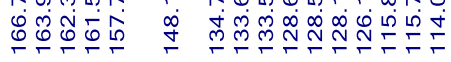

俩, i

ำด ำ

isio

ㅇํำ

फ่ำ

$\stackrel{\circ}{\circ}$

N 111

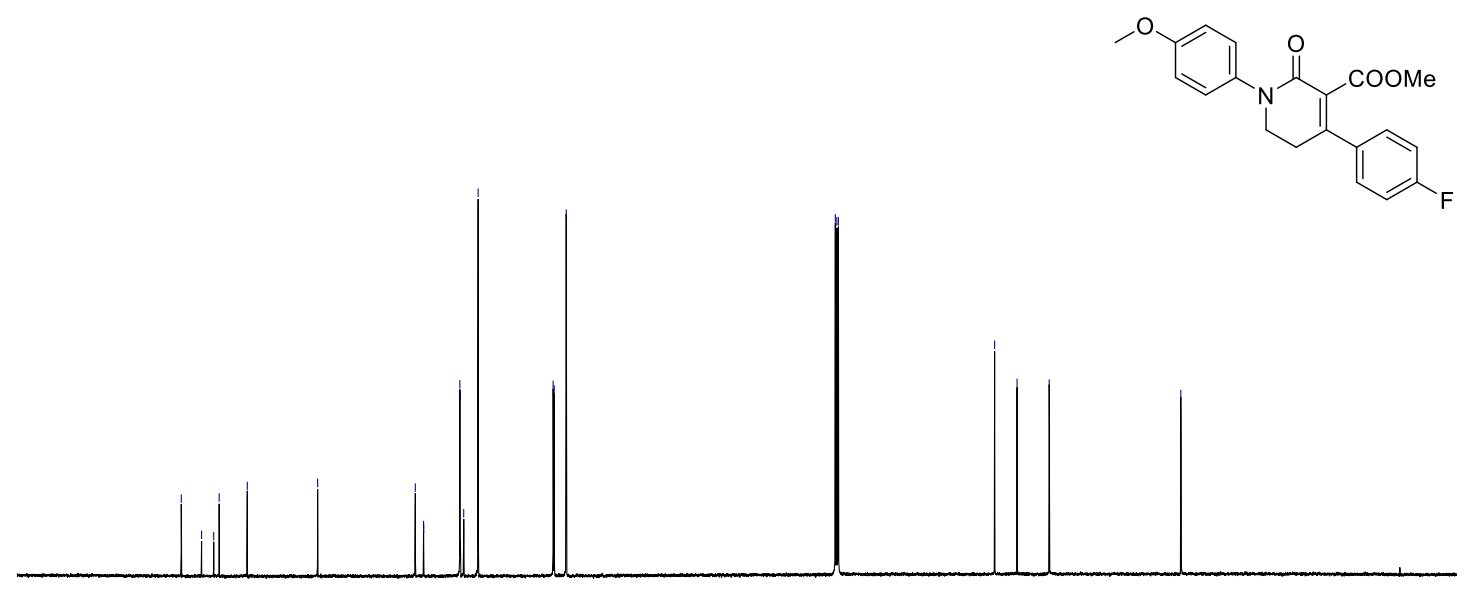

$180 \quad 170$

$1^{\prime} 60^{\prime} 1^{\prime} 50^{\prime} 140^{\prime} 130^{\prime} 120$

10090

$\frac{10}{70} \cdot \frac{1}{80} \cdot 50$

40

$0^{1} 20^{1}+10^{\prime}$ 
${ }^{1} \mathrm{H}$ NMR (400 $\mathrm{MHz}, \mathrm{CDCl}_{3}$ ) of compound $1 \mathrm{e}$

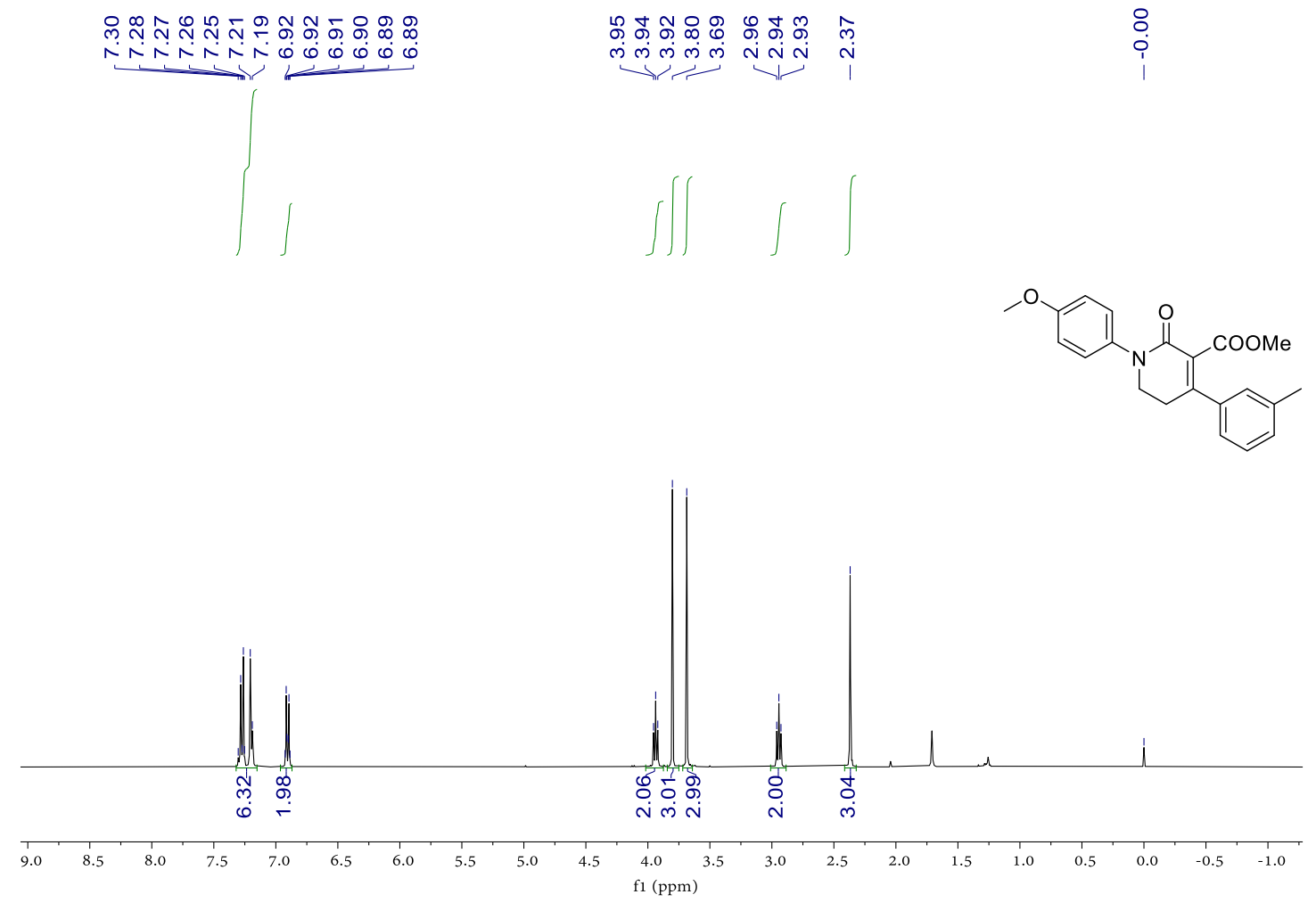

${ }^{13} \mathrm{C}\left\{{ }^{1} \mathrm{H}\right\}$ NMR (151 MHz, $\left.\mathrm{CDCl}_{3}\right)$ of compound $1 \mathrm{e}$

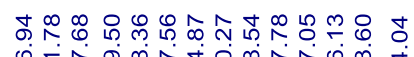

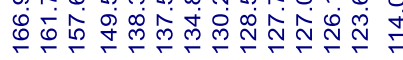

ำ

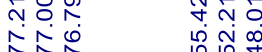

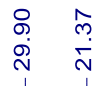

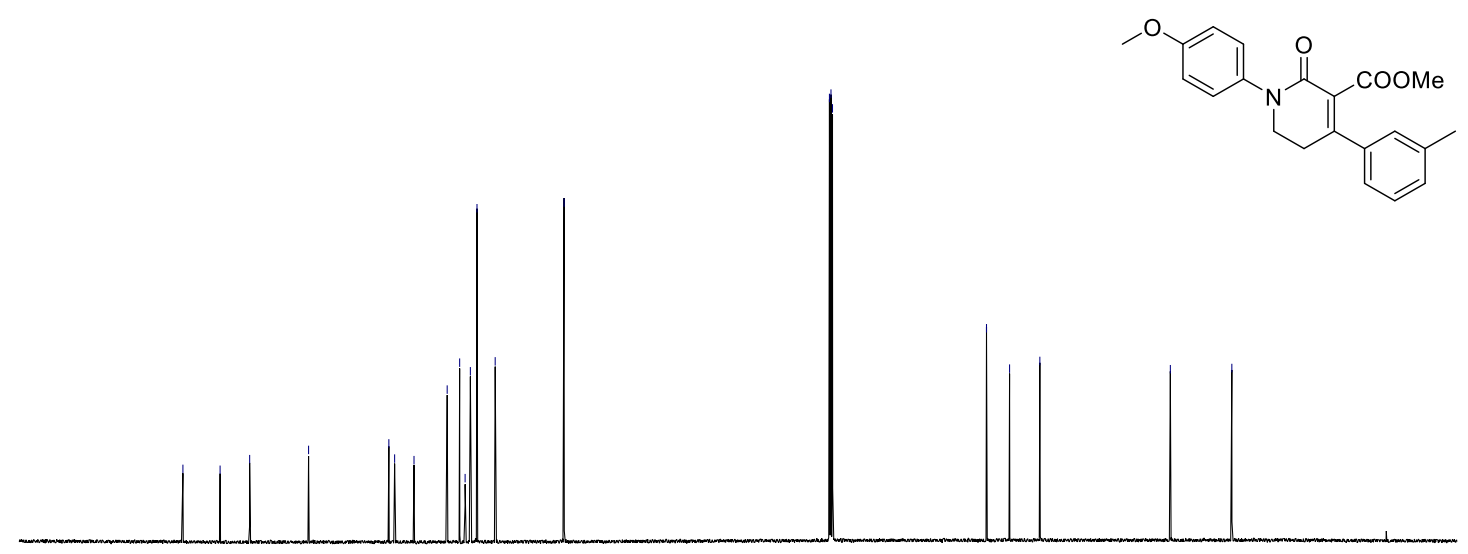

180

$170 \quad 160 \quad 150$

$140 \quad 130$

$120 \quad 110$

$100 \begin{gathered}90 \\ \mathrm{f}_{1}(\mathrm{ppm})\end{gathered}$

$60 \quad 50$

40

30 
${ }^{1} \mathrm{H}$ NMR (400 MHz, $\mathrm{CDCl}_{3}$ ) of compound $1 \mathrm{f}$

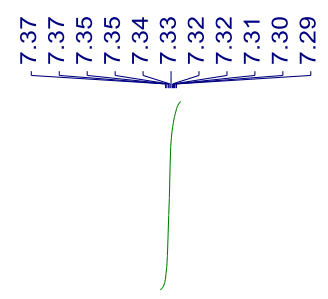

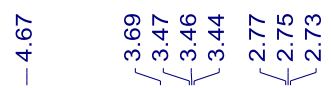

8
$i$
$i$
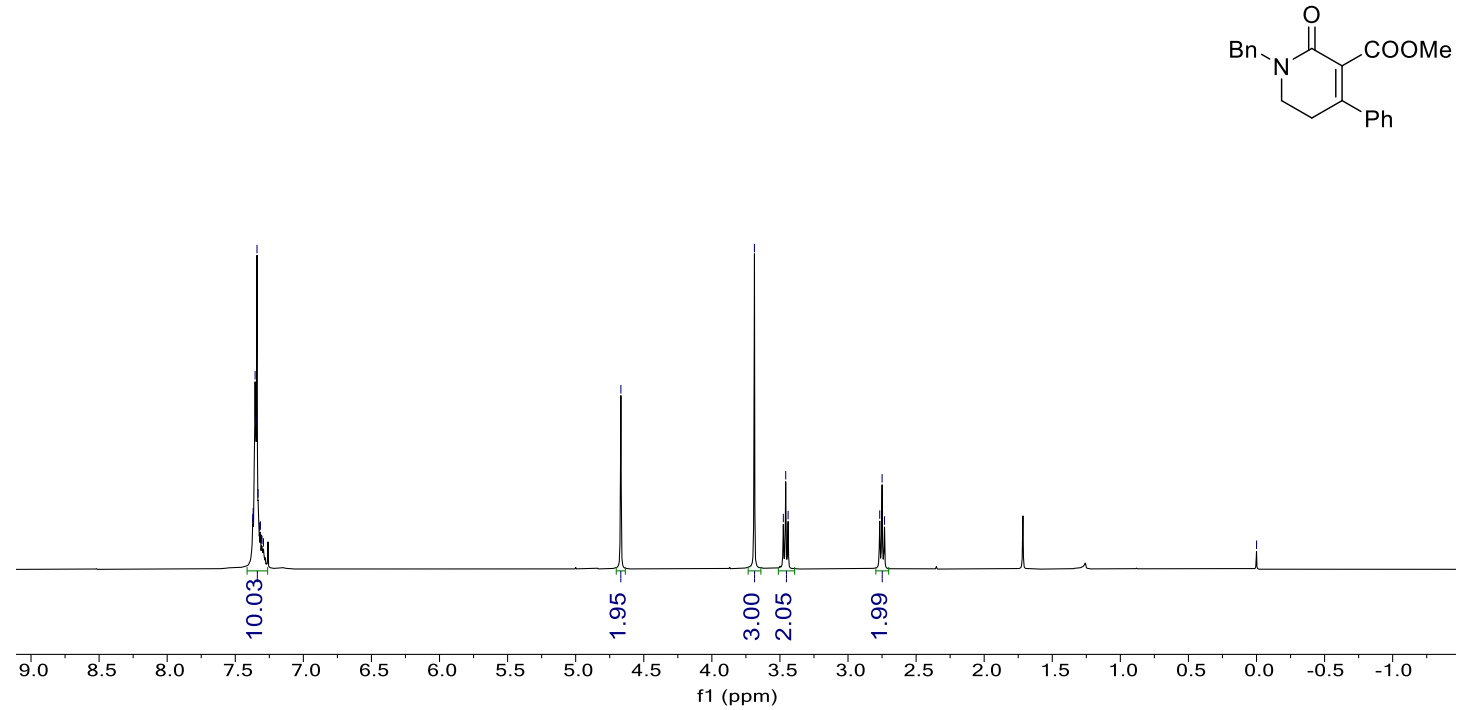

${ }^{13} \mathrm{C}\left\{{ }^{1} \mathrm{H}\right\}$ NMR (101 MHz, $\left.\mathrm{CDCl}_{3}\right)$ of compound 1f

\begin{tabular}{|c|c|c|}
\hline 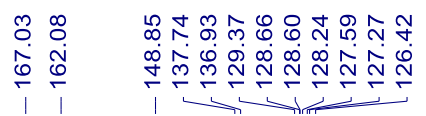 & 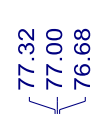 & 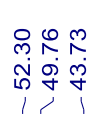 \\
\hline
\end{tabular}

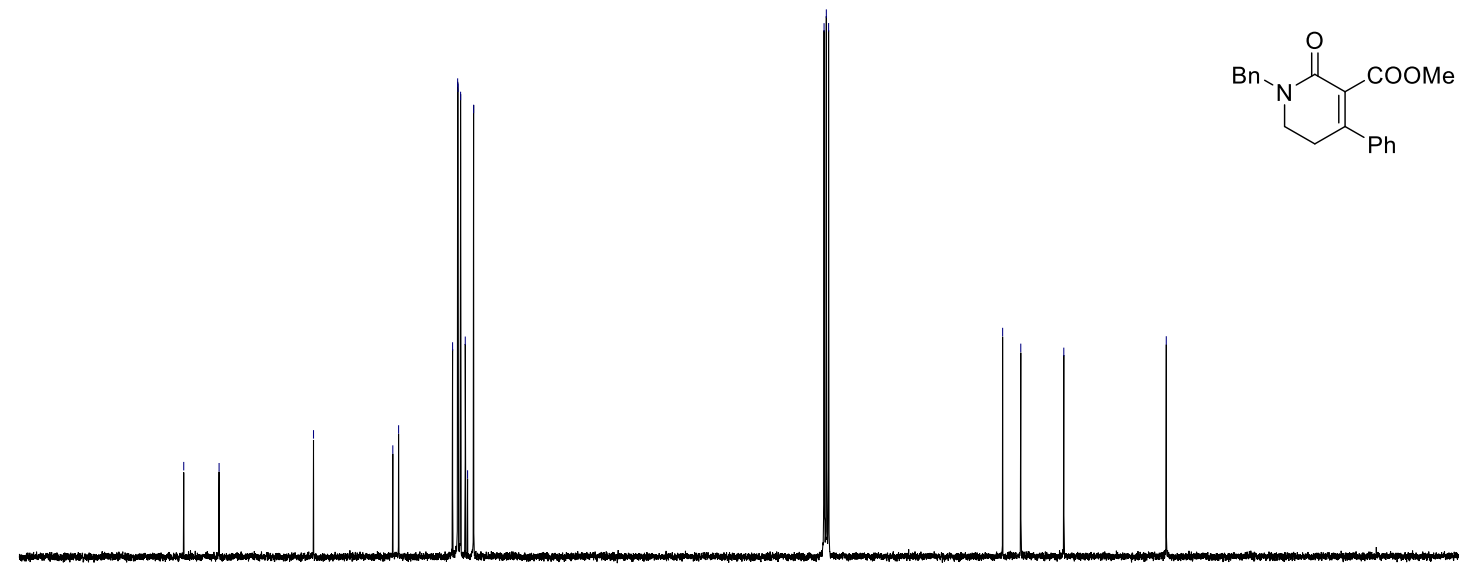

\begin{tabular}{lllllllllllllllllllll}
\hline 90 & 180 & 170 & 160 & 150 & 140 & 130 & 120 & 110 & 100 & $\begin{array}{c}1 \\
90 \\
\mathrm{f} 1(\mathrm{ppm})\end{array}$ & 80 & 70 & 60 & 50 & 40 & 30 & 20 & 10 & 0 & -1
\end{tabular} 


\section{${ }^{1} \mathrm{H}$ NMR (600 MHz, $\mathrm{CDCl}_{3}$ ) of compound $1 \mathrm{~g}$}
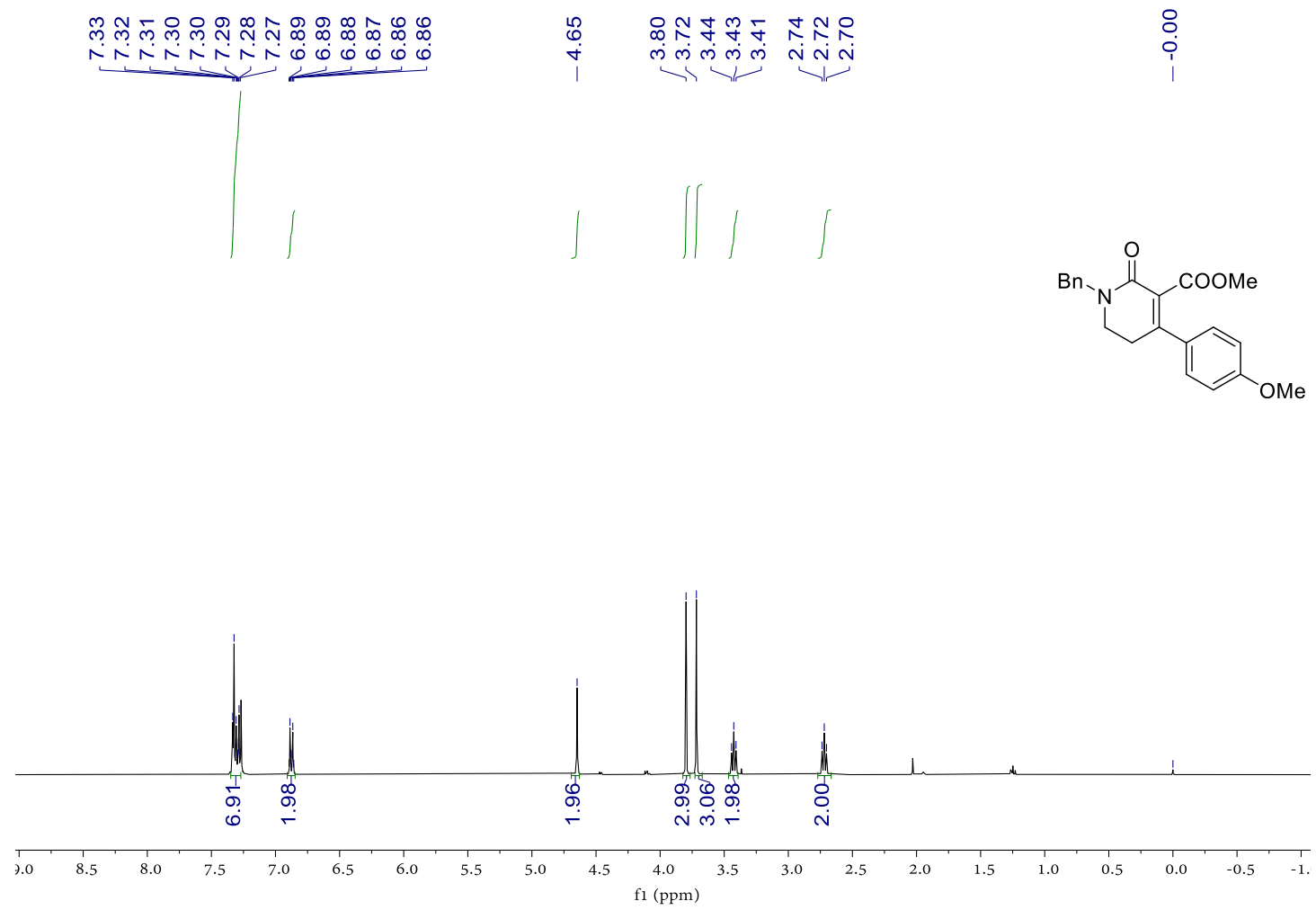

${ }^{13} \mathrm{C}\left\{{ }^{1} \mathrm{H}\right\}$ NMR (101 MHz, $\left.\mathrm{CDCl}_{3}\right)$ of compound $1 \mathrm{~g}$

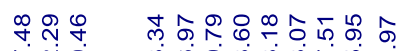

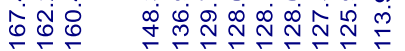

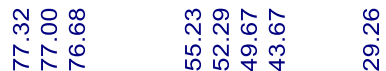

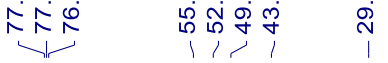
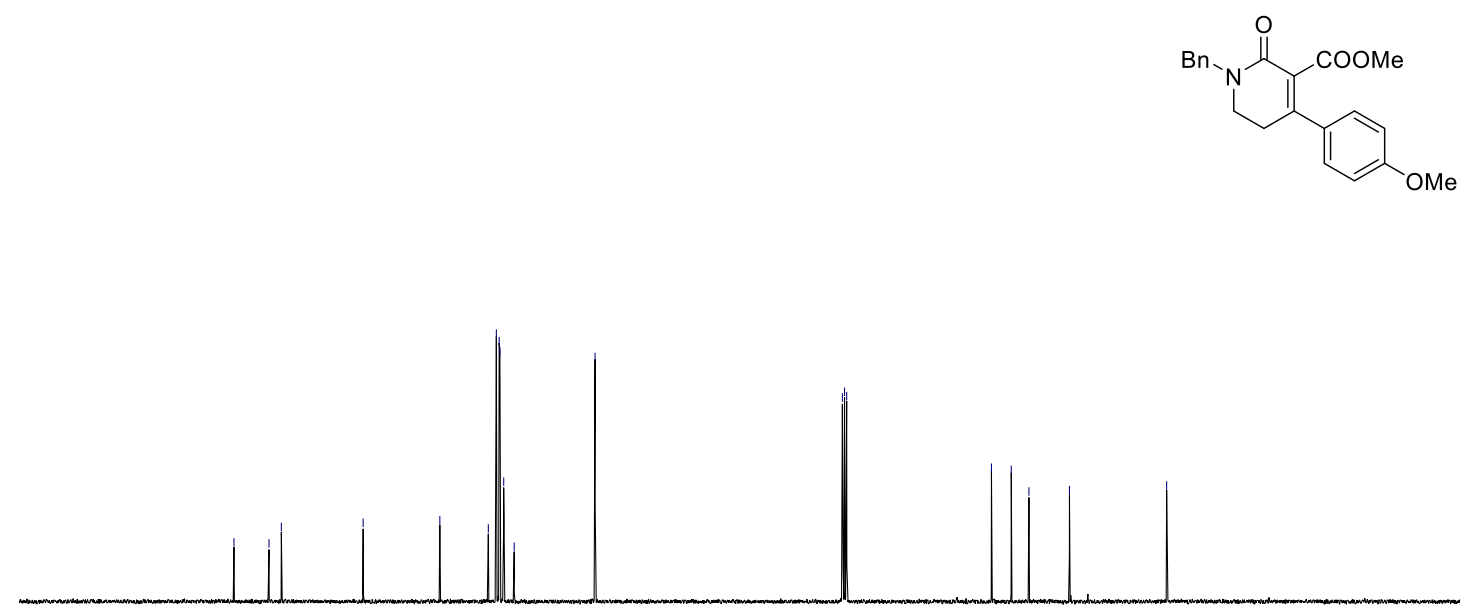

$\begin{array}{lllllllllll}190 & 180 & 170 & 160 & 150 & 140 & 130 & 120 & 110 & 100 & 90 \\ \text { f1 }(\mathrm{ppm})\end{array}$ 


\section{${ }^{1} \mathrm{H}$ NMR (400 MHz, $\mathrm{CDCl}_{3}$ ) of compound $1 \mathrm{~h}$}

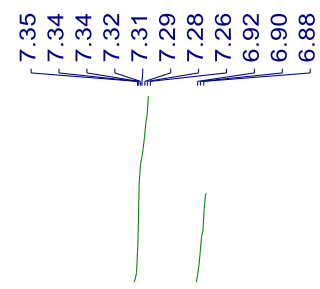

•)

웅
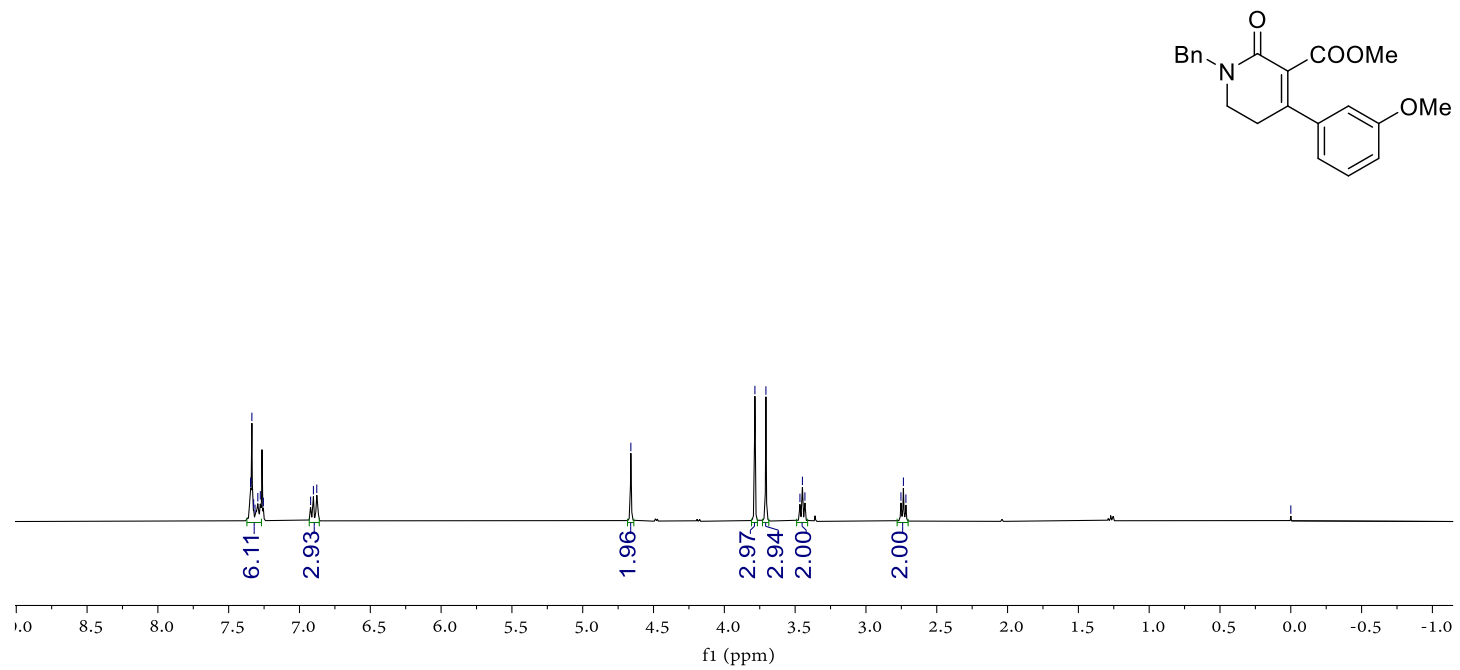

${ }^{13} \mathrm{C}\left\{{ }^{1} \mathrm{H}\right\}$ NMR (151 MHz, $\left.\mathrm{CDCl}_{3}\right)$ of compound $1 \mathrm{~h}$

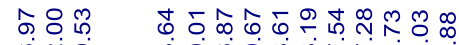

\& ن

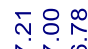

章定

ㅇำ용

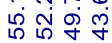

$\stackrel{\infty}{\substack{\infty}}$

$1,1<1$
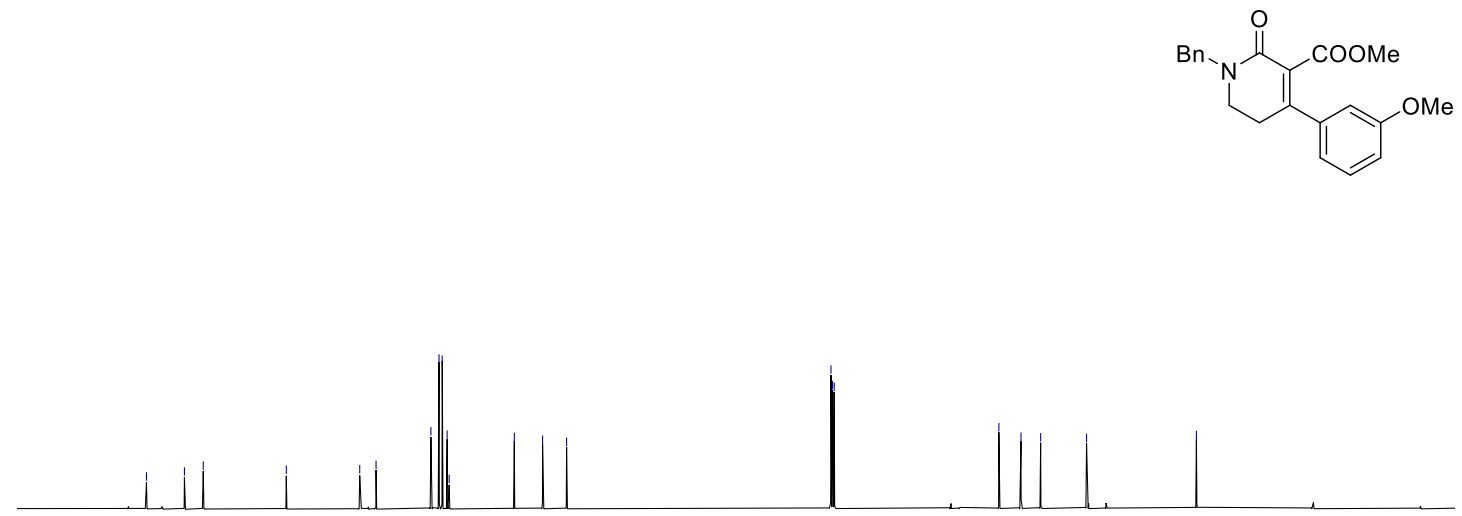
${ }^{1} \mathrm{H}$ NMR (400 MHz, $\mathrm{CDCl}_{3}$ ) of compound 1i

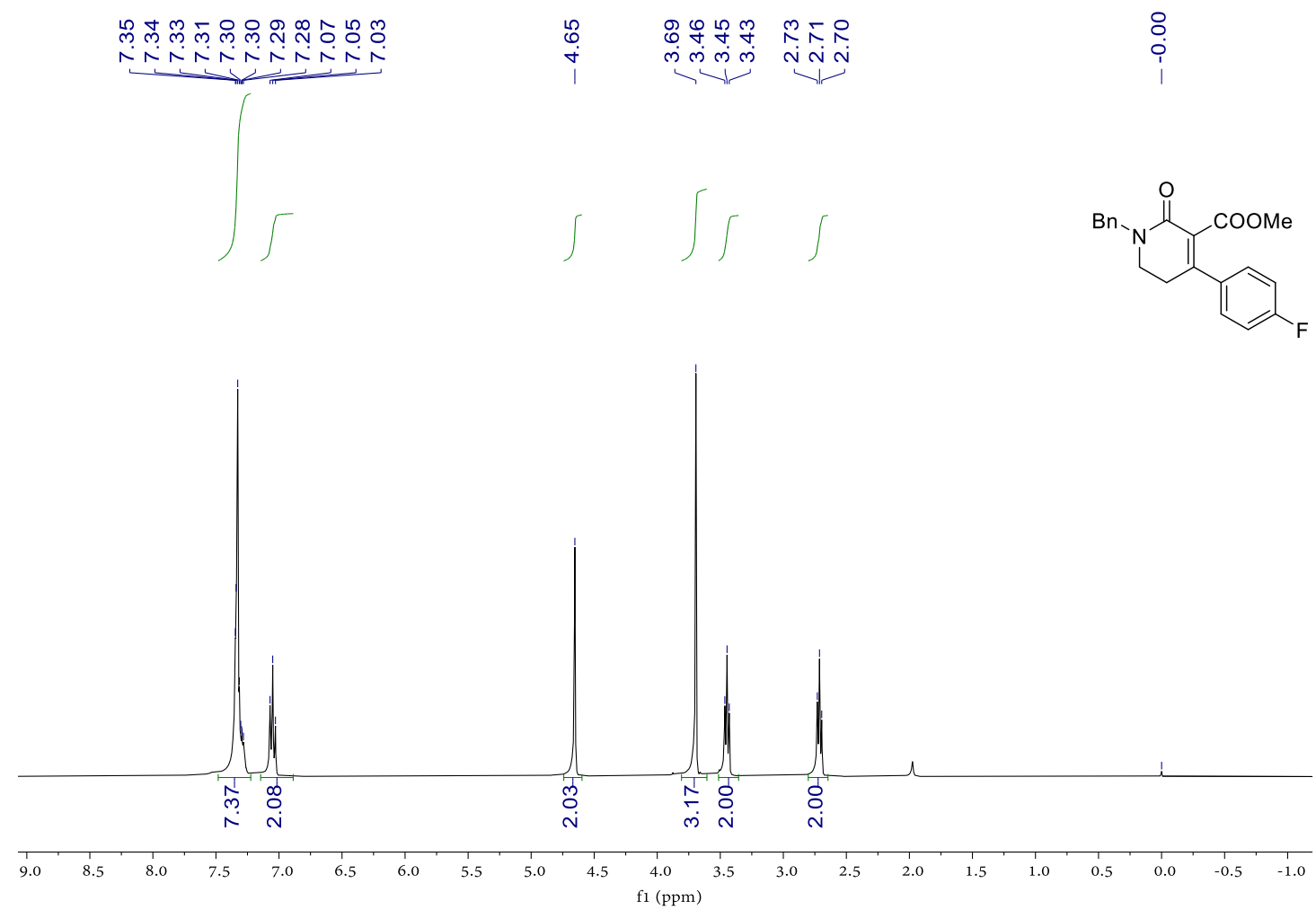

${ }^{19}$ F NMR (376 MHz, $\mathrm{CDCl}_{3}$ ) of compound $1 \mathrm{i}$
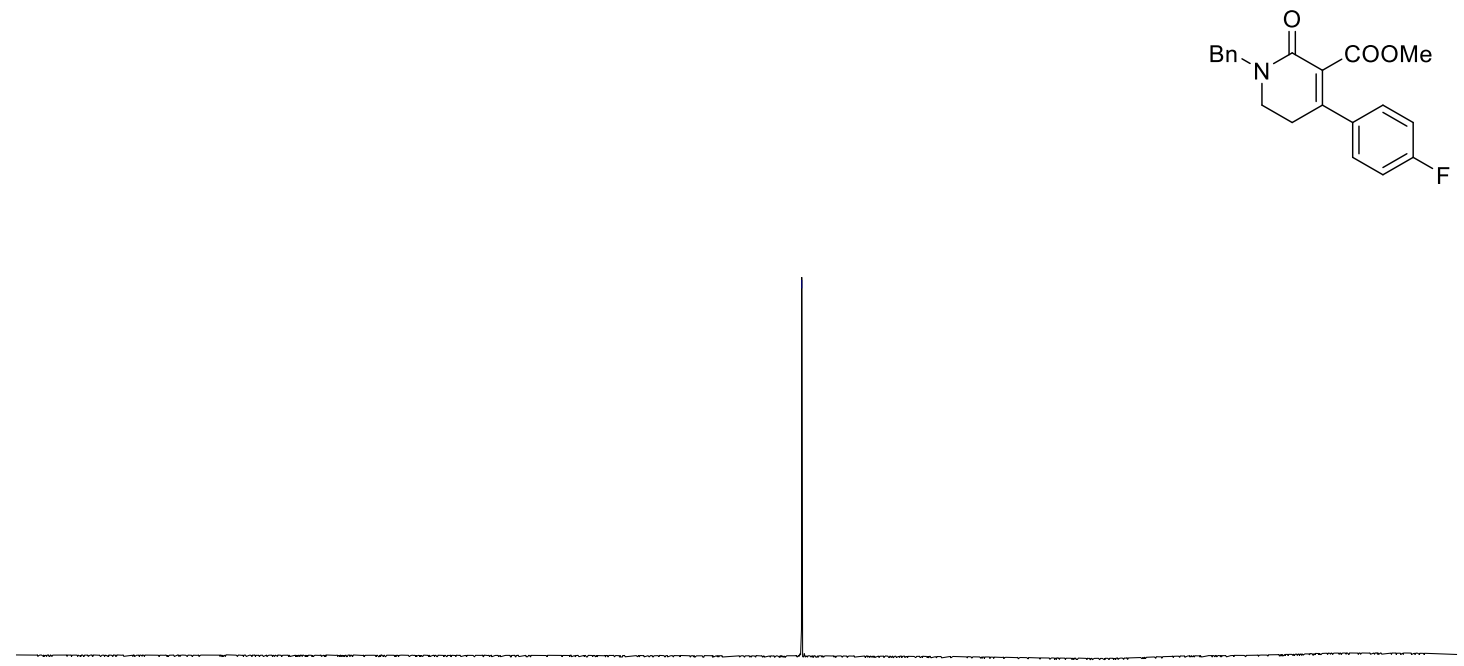

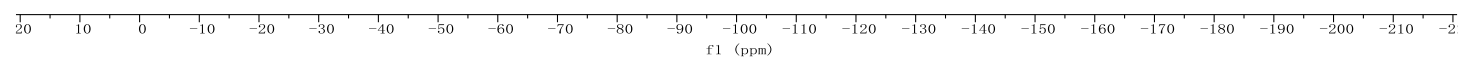


${ }^{13} \mathrm{C}\left\{{ }^{1} \mathrm{H}\right\}$ NMR (151 MHz, $\left.\mathrm{CDCl}_{3}\right)$ of compound 1i

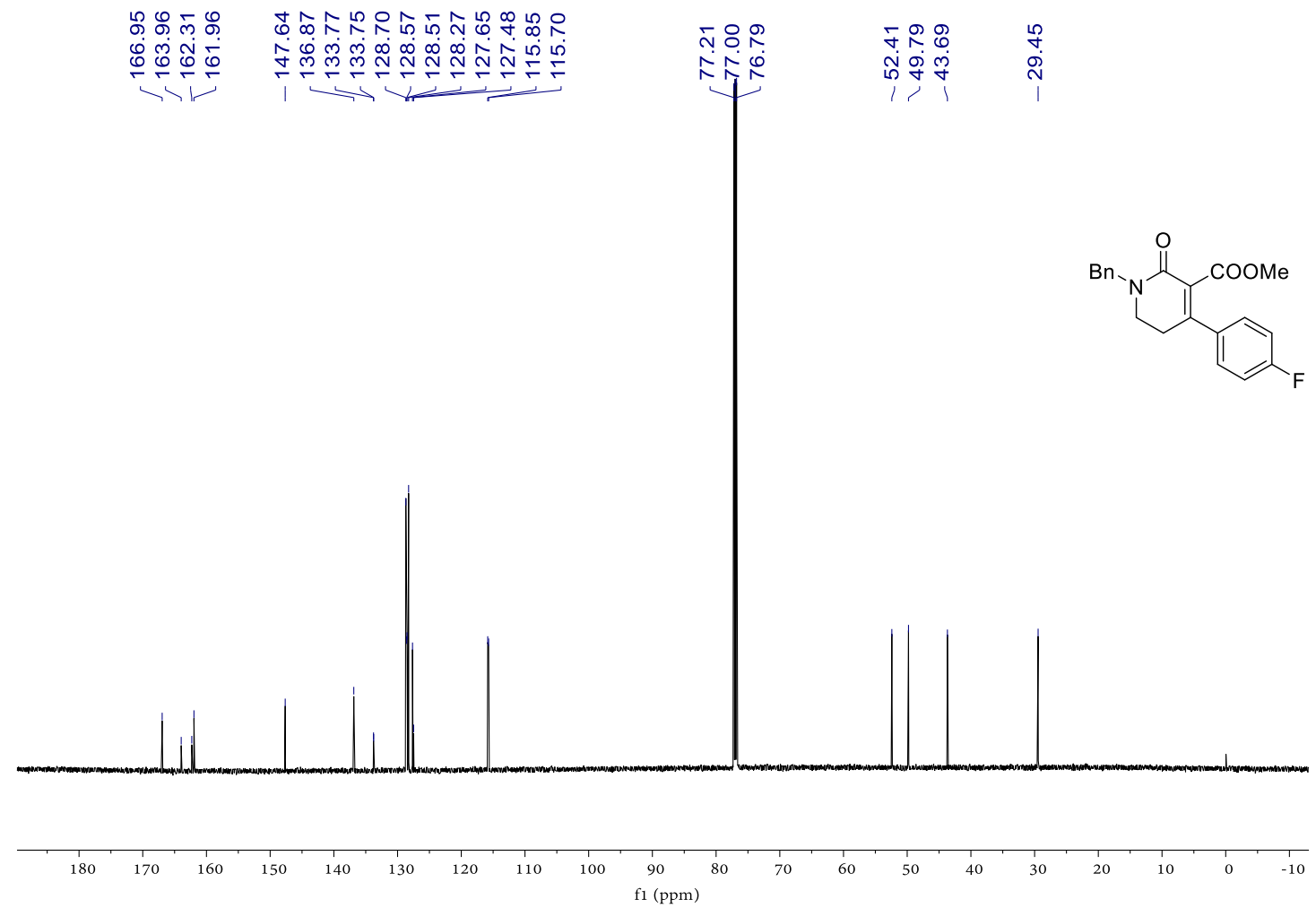




\section{${ }^{1} \mathrm{H}$ NMR (400 MHz, $\left.\mathrm{CDCl}_{3}\right)$ of compound $1 \mathrm{j}$}

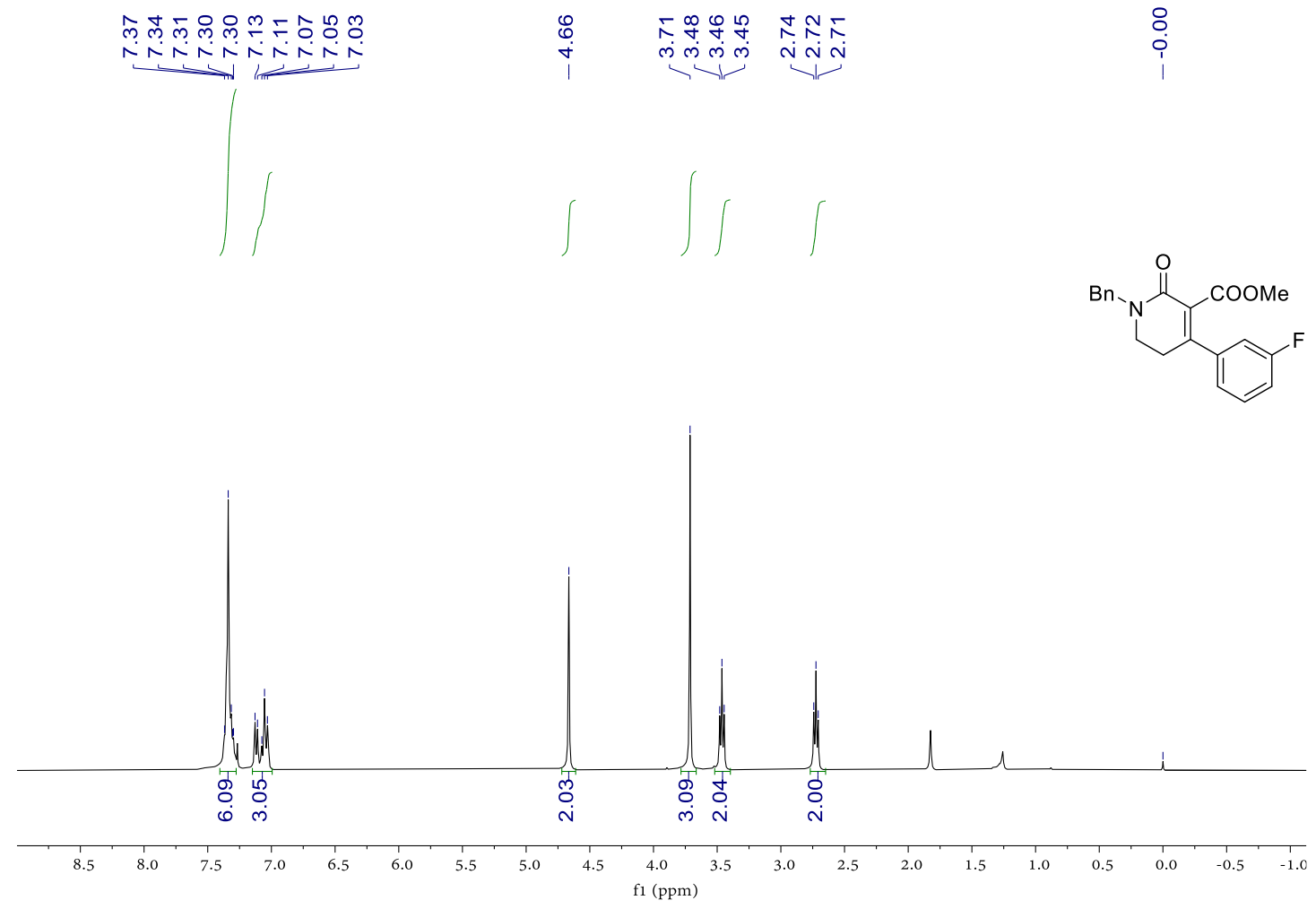

${ }^{19}$ F NMR (376 MHz, $\mathrm{CDCl}_{3}$ ) of compound $1 \mathrm{j}$
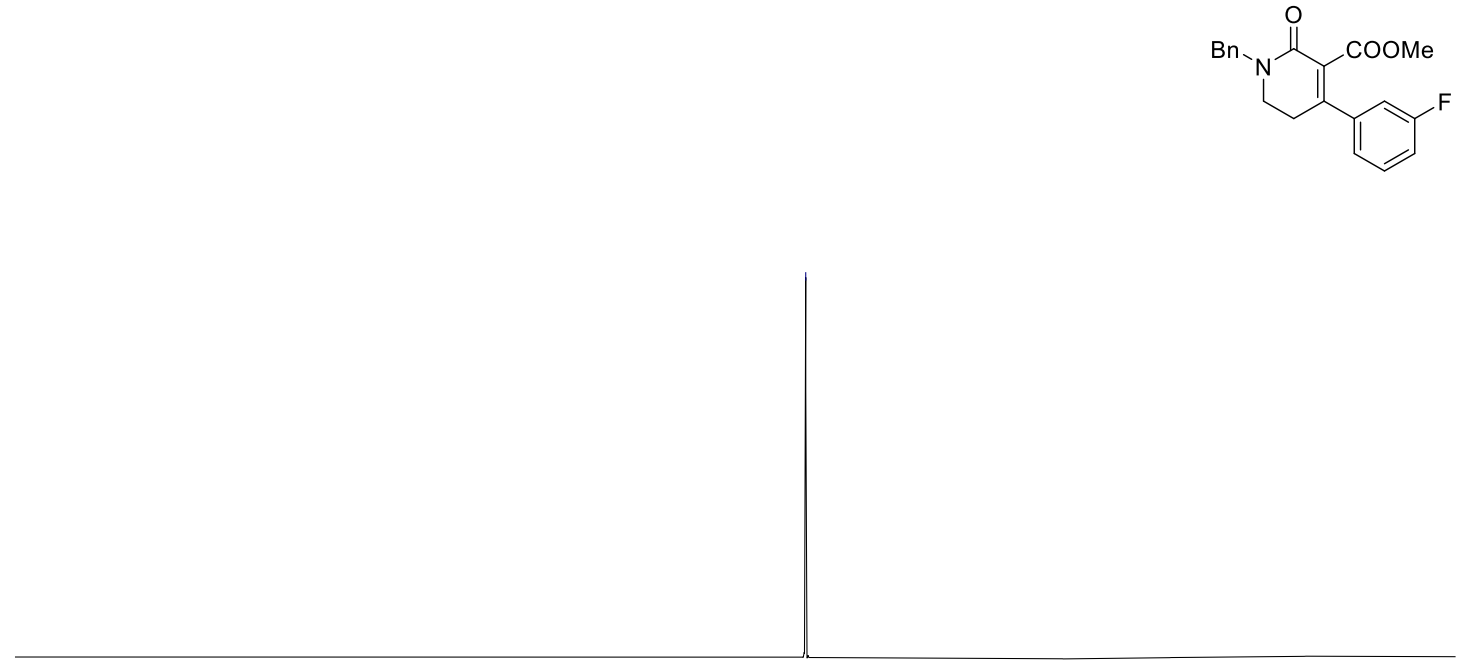

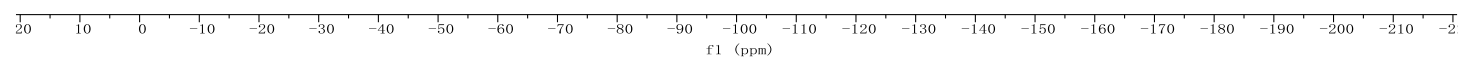




\section{${ }^{13} \mathrm{C}\left\{{ }^{1} \mathrm{H}\right\}$ NMR (101 MHz, $\left.\mathrm{CDCl}_{3}\right)$ of compound $1 \mathrm{j}$}

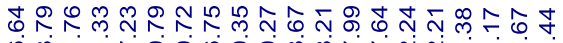

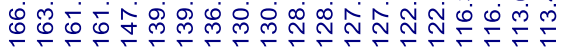

잉 8

䄈

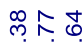

$\stackrel{\infty}{\stackrel{\infty}{\infty}}$

ᄂit

îd

$\stackrel{\infty}{\stackrel{\infty}{\infty}}$
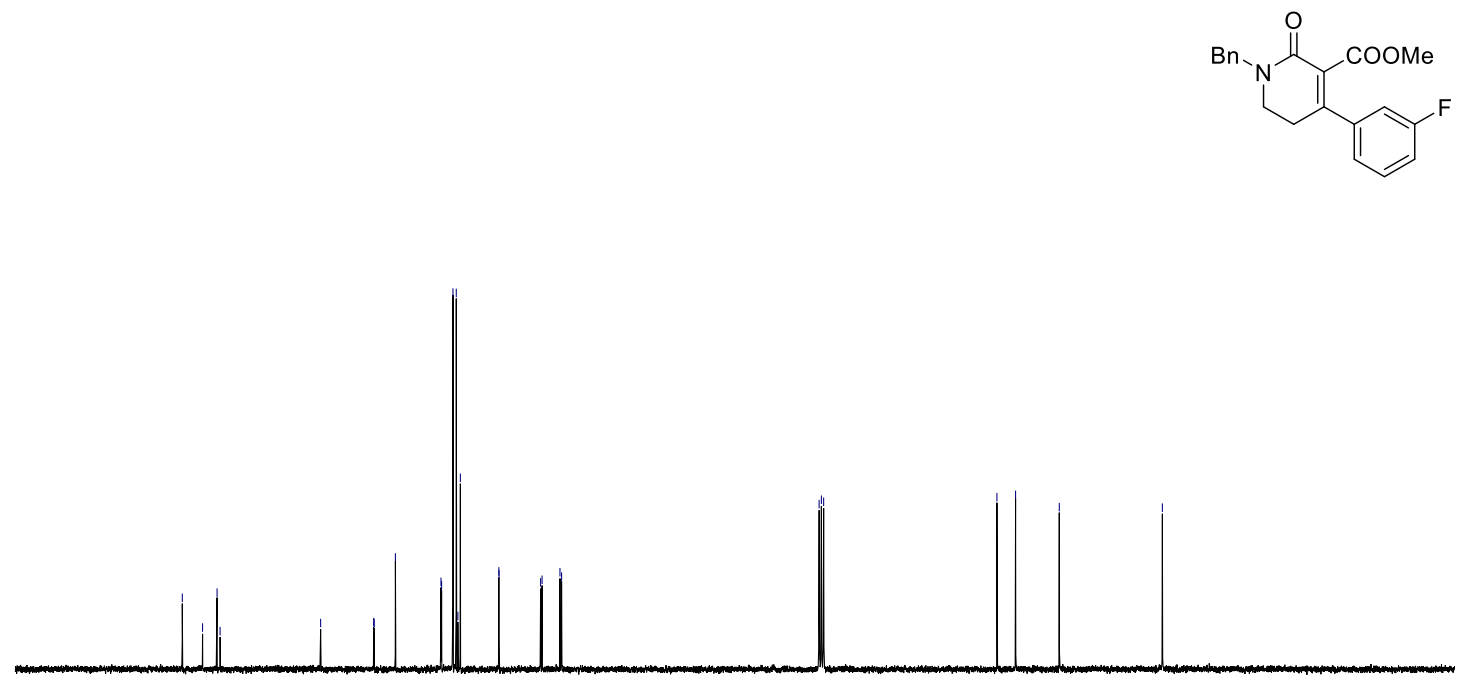

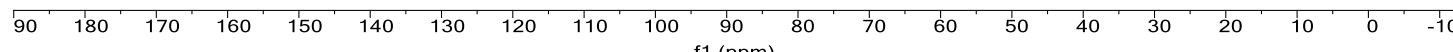


${ }^{1} \mathrm{H}$ NMR (400 MHz, $\mathrm{CDCl}_{3}$ ) of compound $1 \mathrm{k}$

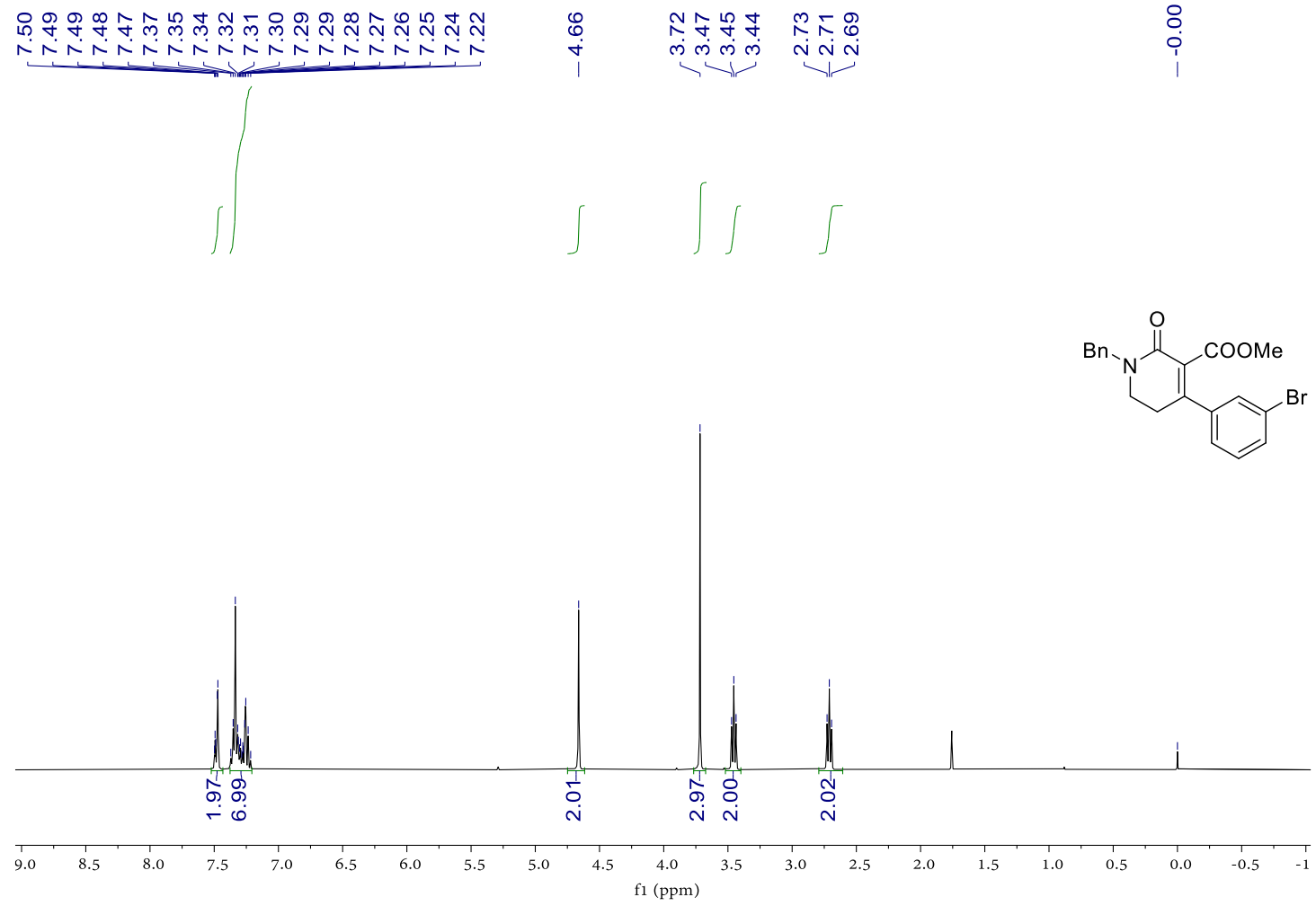

${ }^{13} \mathrm{C}\left\{{ }^{1} \mathrm{H}\right\}$ NMR (101 MHz, $\left.\mathrm{CDCl}_{3}\right)$ of compound $1 \mathrm{k}$

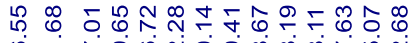

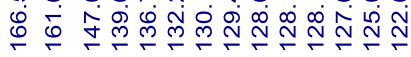

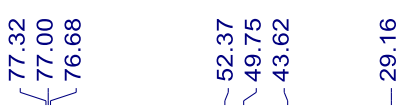

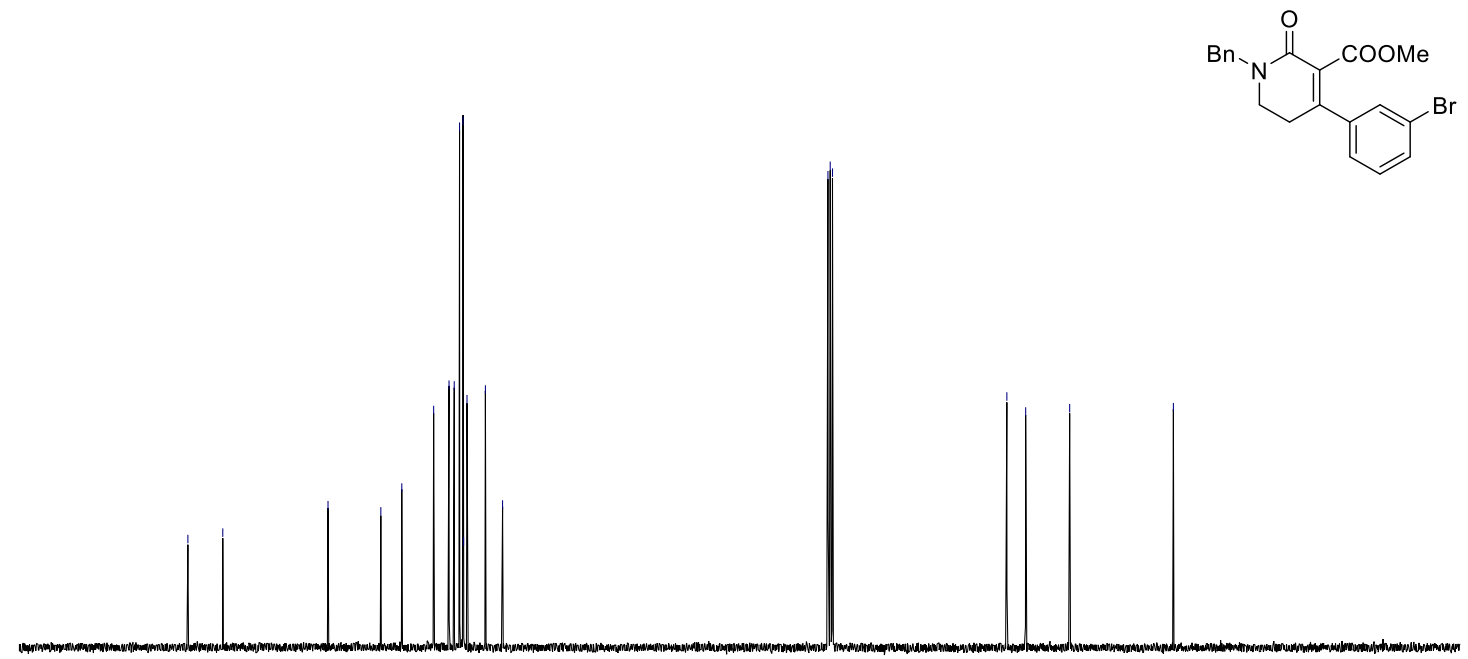


${ }^{1} \mathrm{H}$ NMR (400 MHz, $\mathrm{CDCl}_{3}$ ) of compound 11
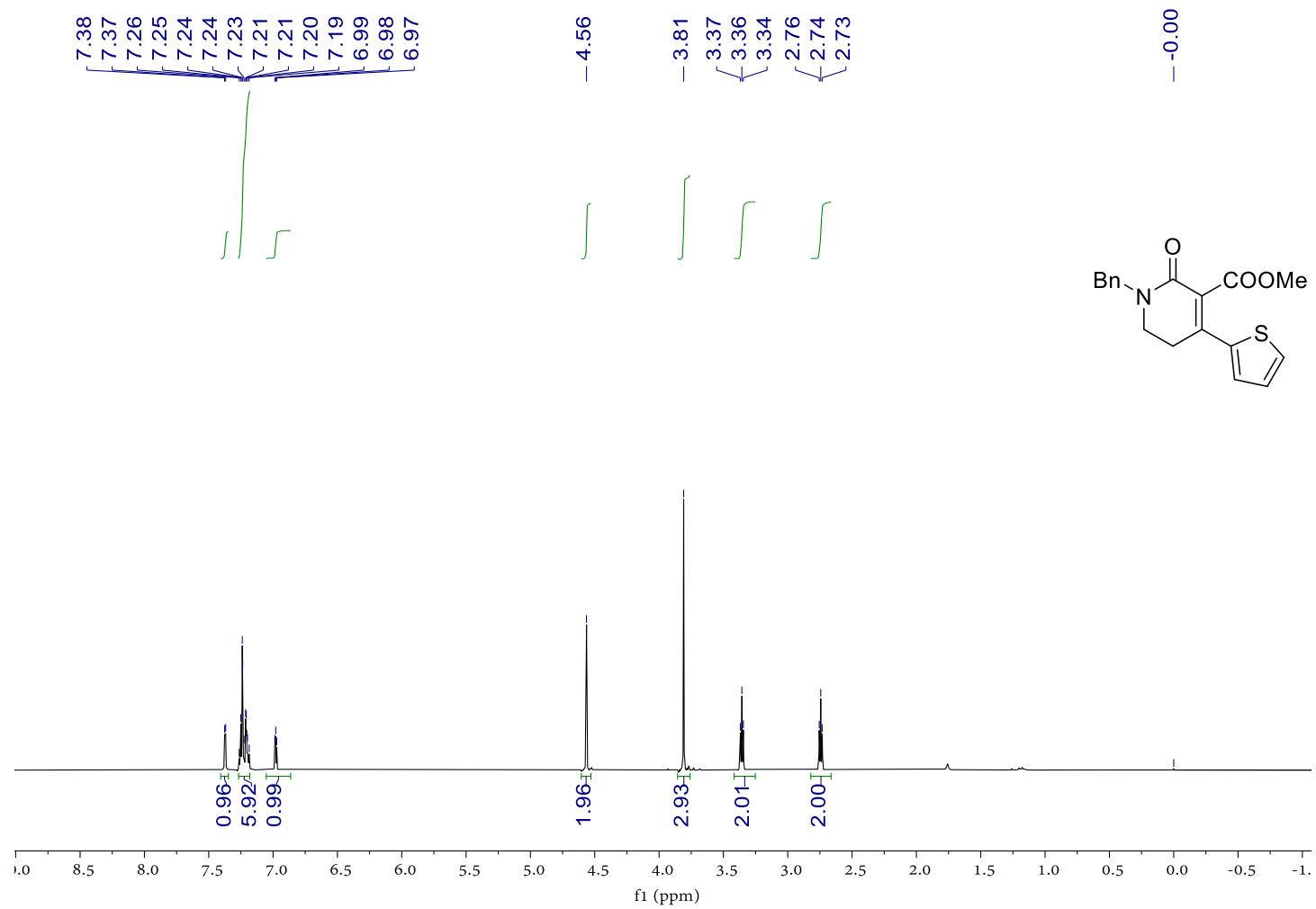

${ }^{13} \mathrm{C}\left\{{ }^{1} \mathrm{H}\right\}$ NMR (101 MHz, $\left.\mathrm{CDCl}_{3}\right)$ of compound 11

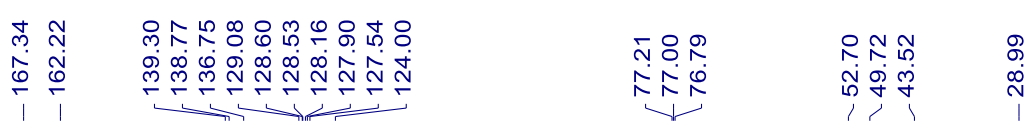

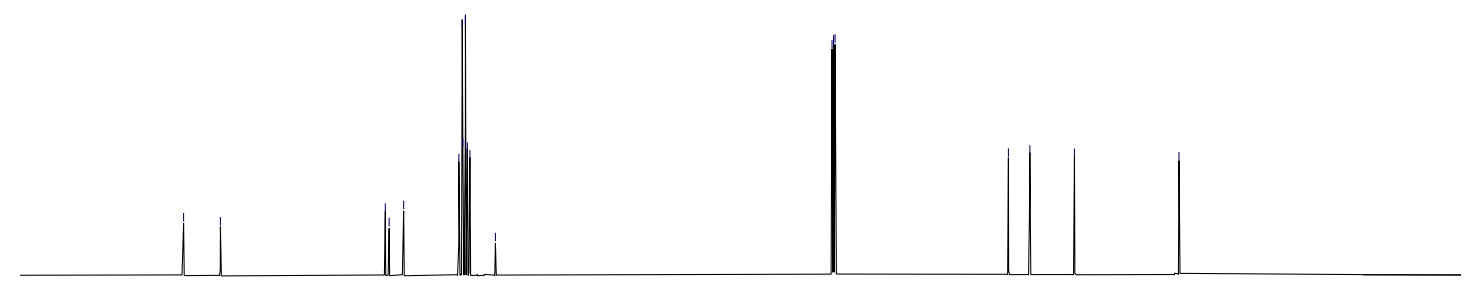


${ }^{1} \mathrm{H}$ NMR (600 MHz, $\left.\mathrm{CDCl}_{3}\right)$ of compound $1 \mathrm{~m}$

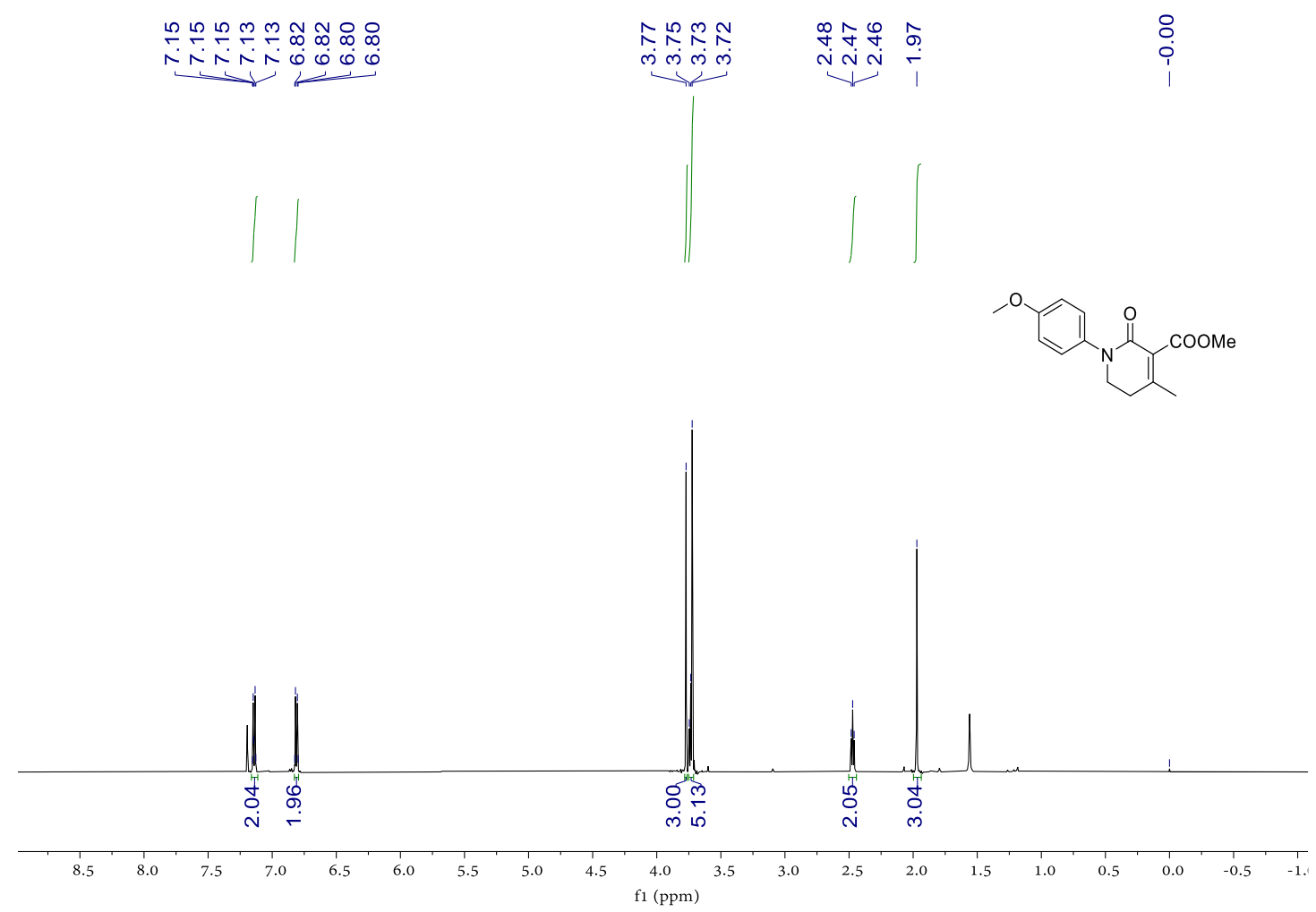

${ }^{13} \mathrm{C}\left\{{ }^{1} \mathrm{H}\right\}$ NMR (151 MHz, $\left.\mathrm{CDCl}_{3}\right)$ of compound $1 \mathrm{~m}$

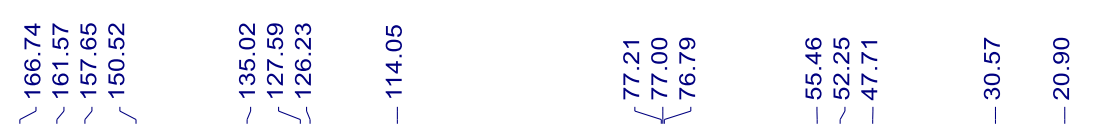

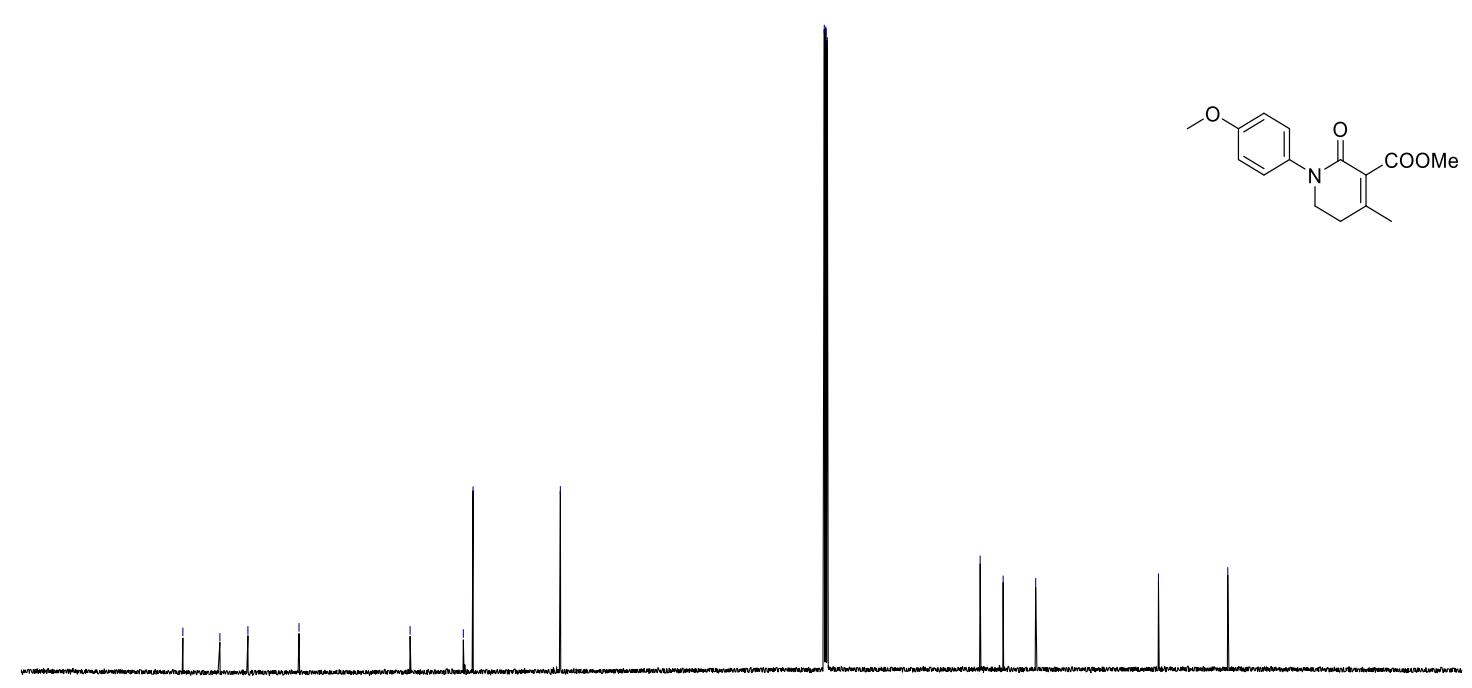

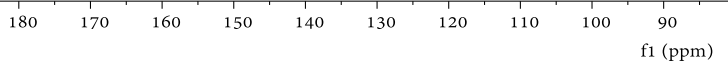




\section{${ }^{1} \mathrm{H}$ NMR (600 MHz, $\mathrm{CDCl}_{3}$ ) of compound 1n}

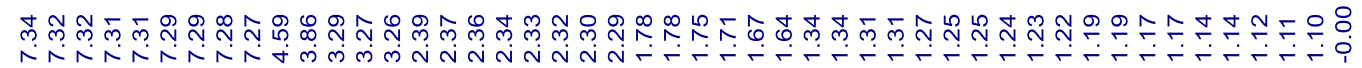
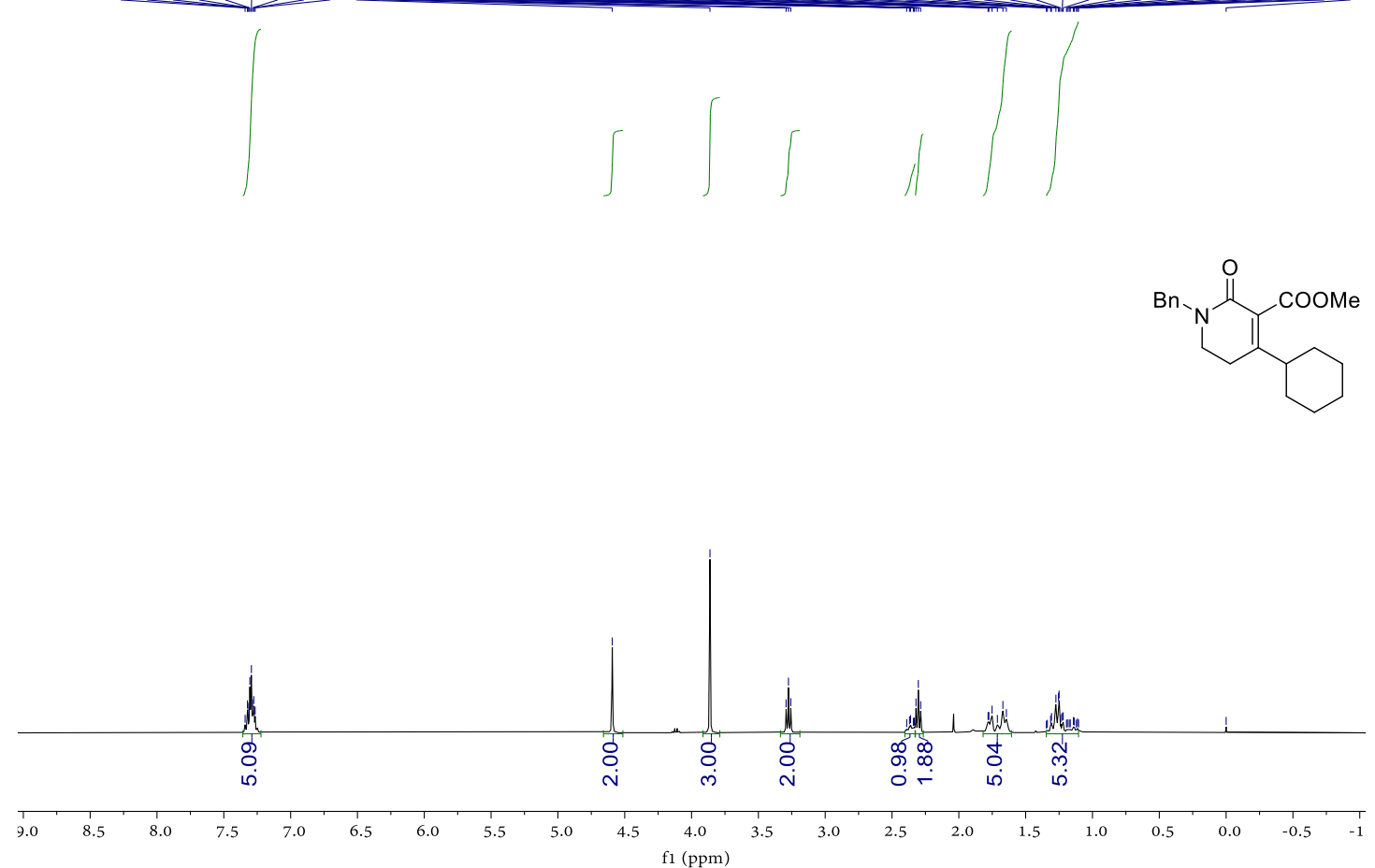

${ }^{13} \mathrm{C}\left\{{ }^{1} \mathrm{H}\right\}$ NMR (151 MHz, $\left.\mathrm{CDCl}_{3}\right)$ of compound 1n

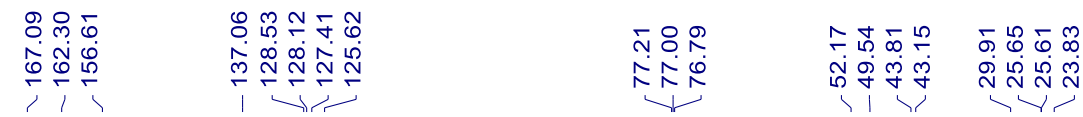

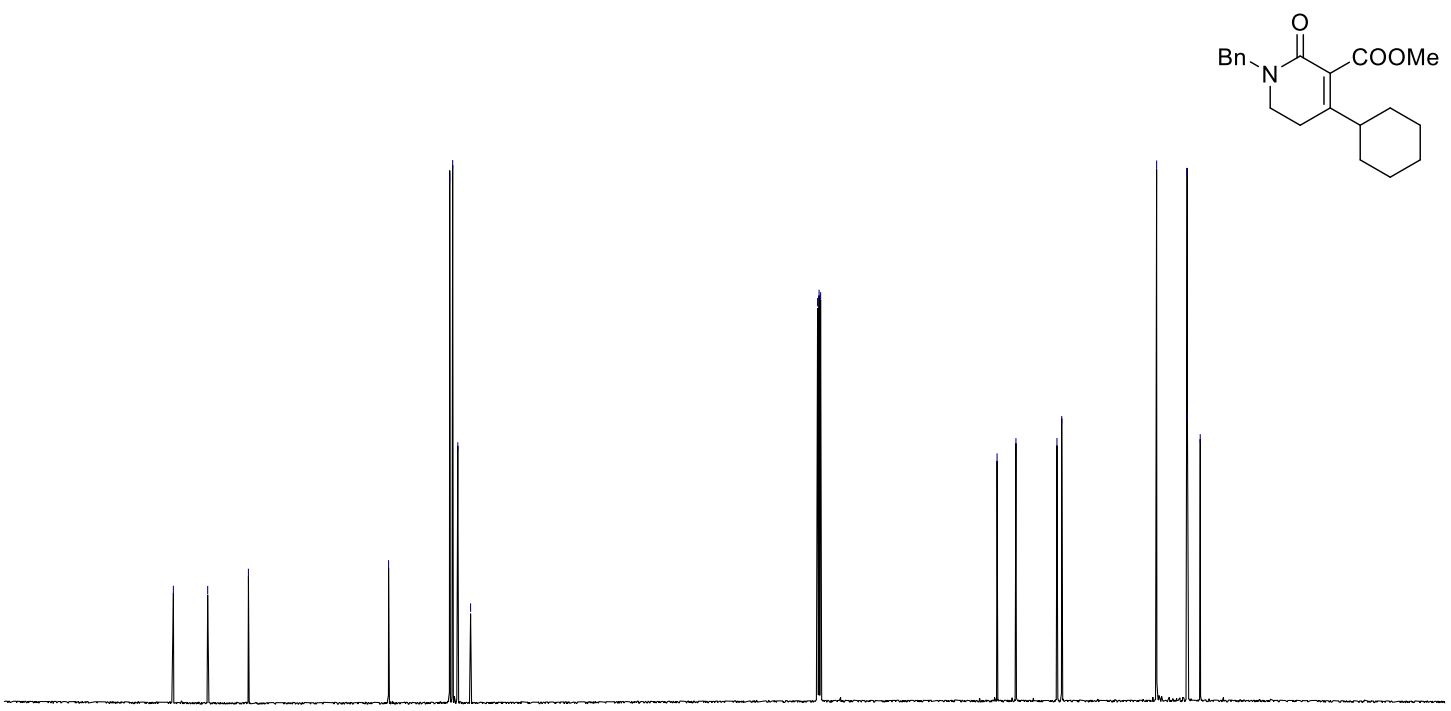

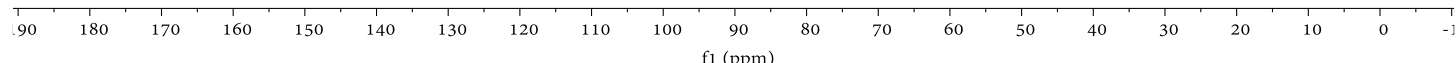


${ }^{1} \mathrm{H}$ NMR (600 MHz, $\left.\mathrm{CDCl}_{3}\right)$ of compound 10

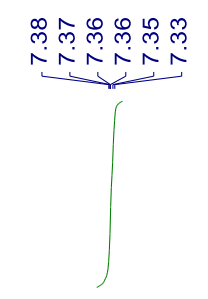

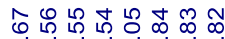

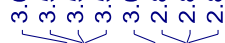

$\stackrel{8}{i}$
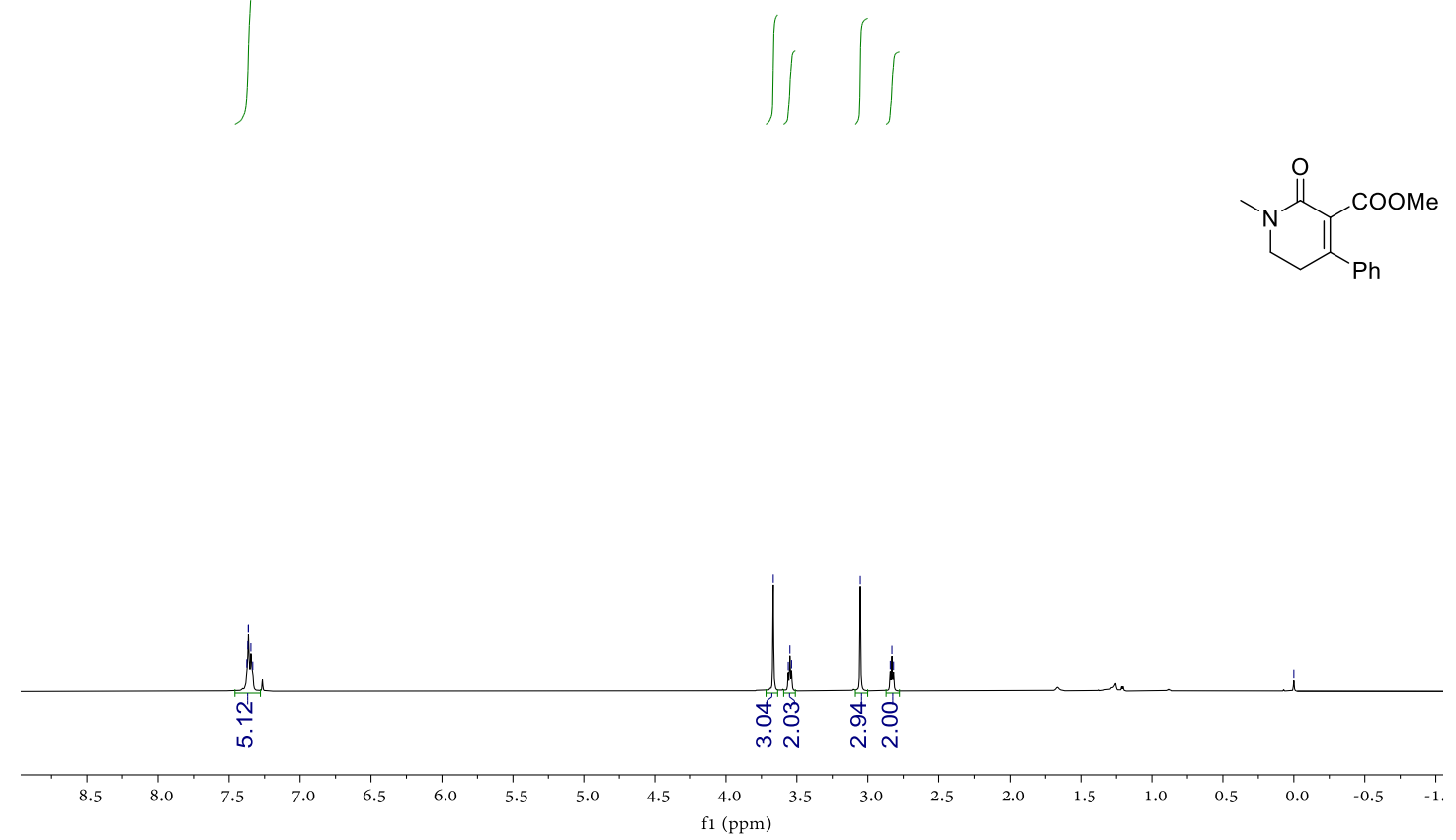

${ }^{13} \mathrm{C}\left\{{ }^{1} \mathrm{H}\right\}$ NMR (151 MHz, $\left.\mathrm{CDCl}_{3}\right)$ of compound 10

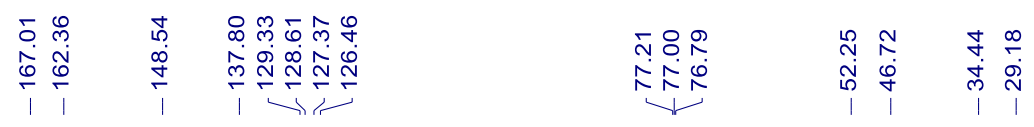

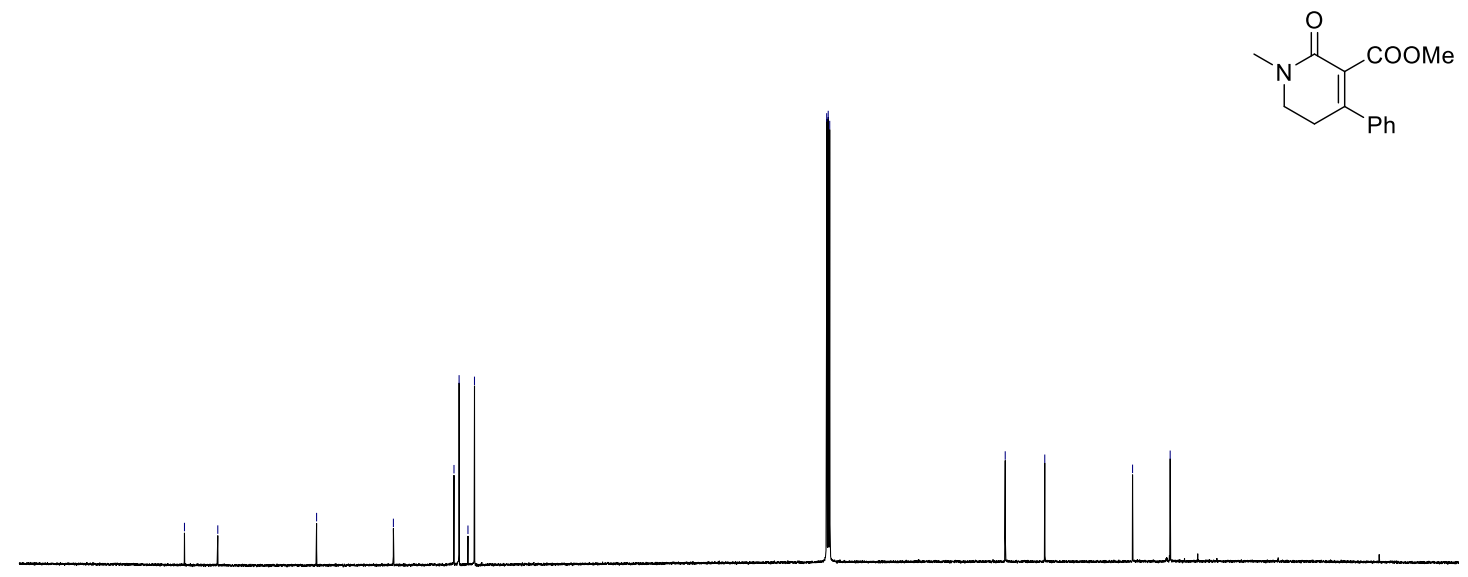

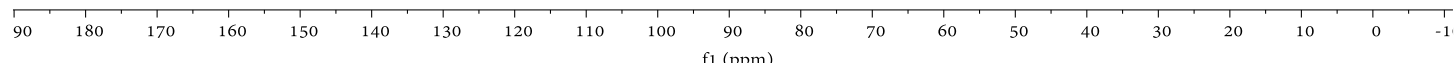


${ }^{1} \mathrm{H}$ NMR (400 MHz, $\mathrm{CDCl}_{3}$ ) of compound 2a

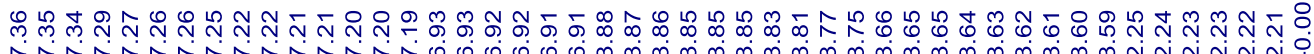

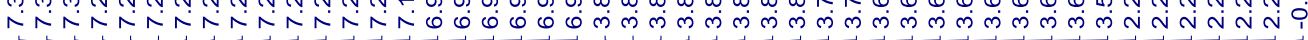
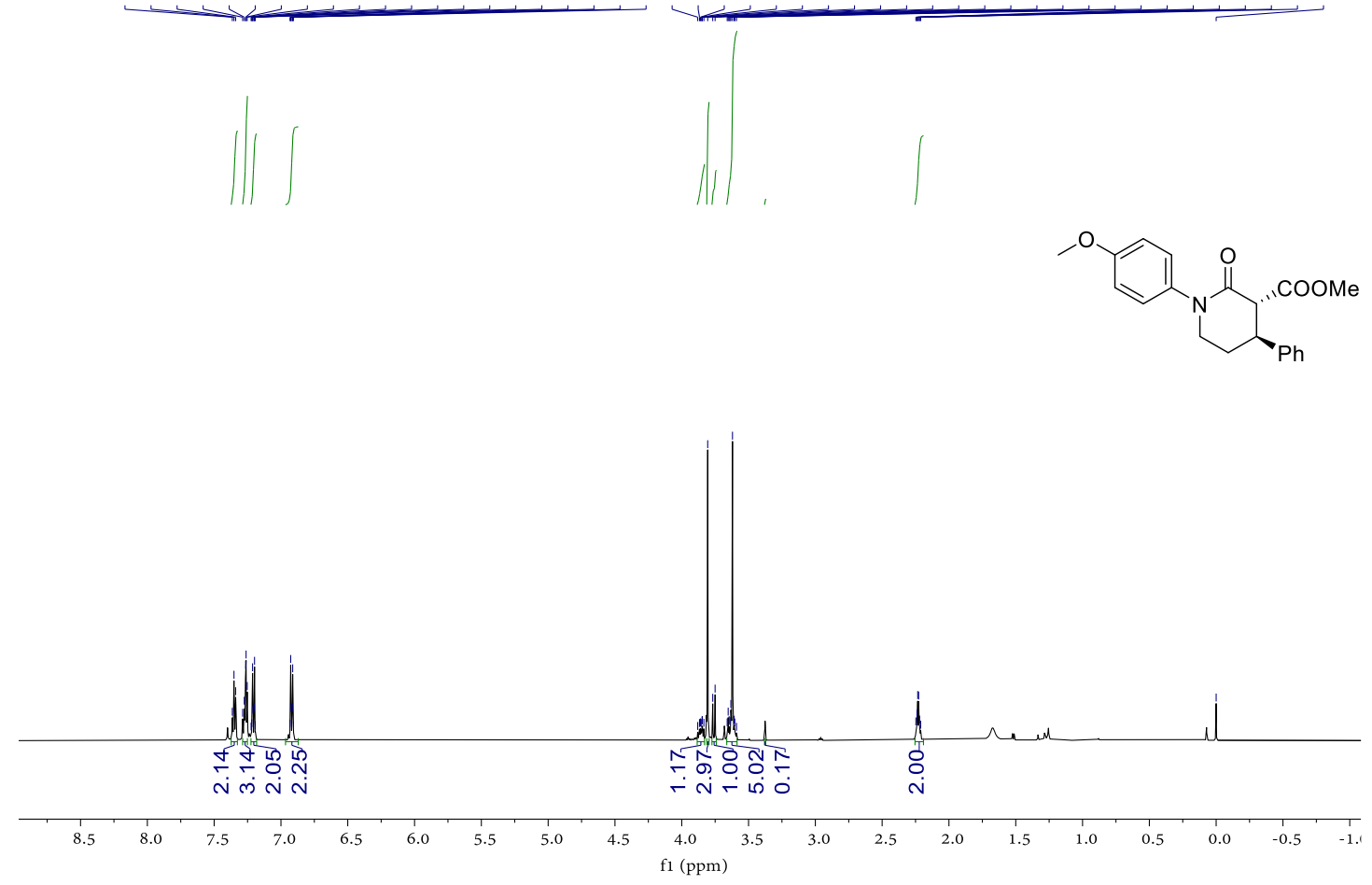

${ }^{13} \mathrm{C}\left\{{ }^{1} \mathrm{H}\right\}$ NMR (101 MHz, $\left.\mathrm{CDCl}_{3}\right)$ of compound 2a

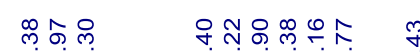

ij

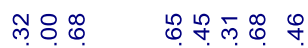

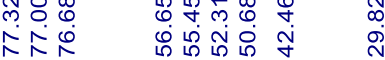

hli i l

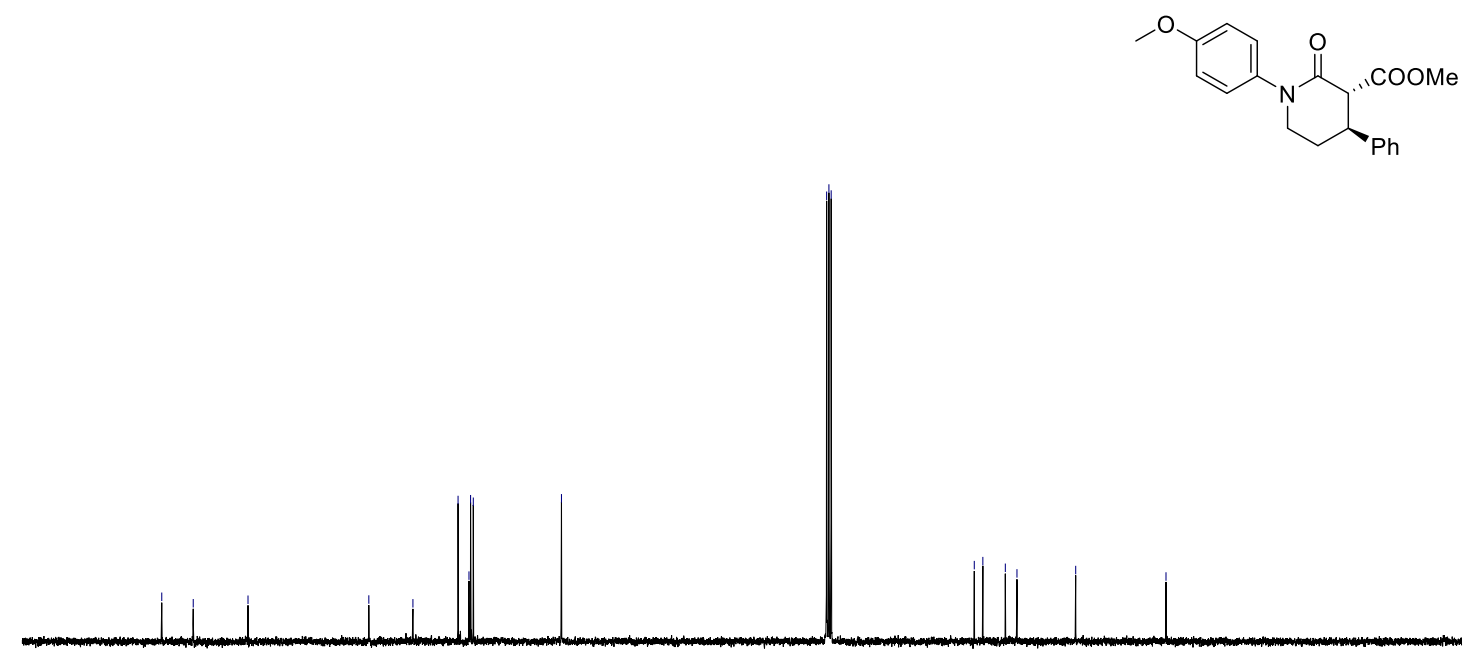

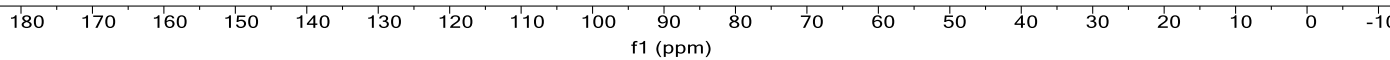




\section{${ }^{1} \mathrm{H}$ NMR (400 MHz, $\mathrm{CDCl}_{3}$ ) of compound 2b}

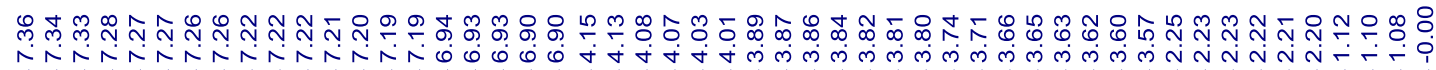
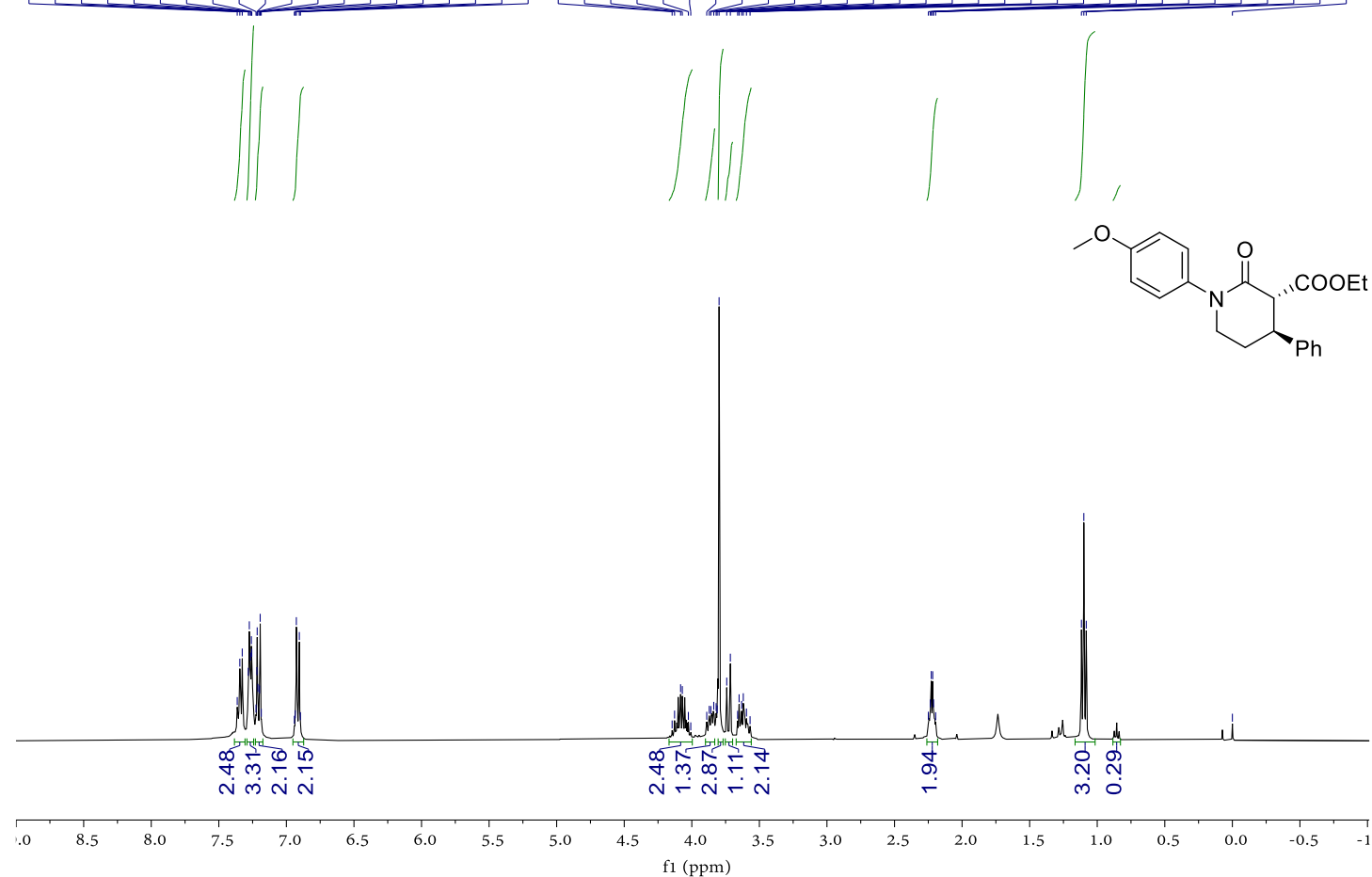

${ }^{13} \mathrm{C}\left\{{ }^{1} \mathrm{H}\right\}$ NMR (101 MHz, $\left.\mathrm{CDCl}_{3}\right)$ of compound $2 \mathrm{~b}$

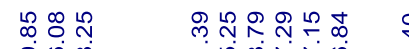

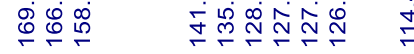

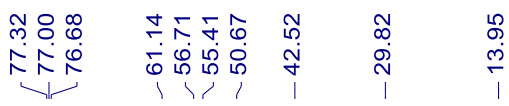
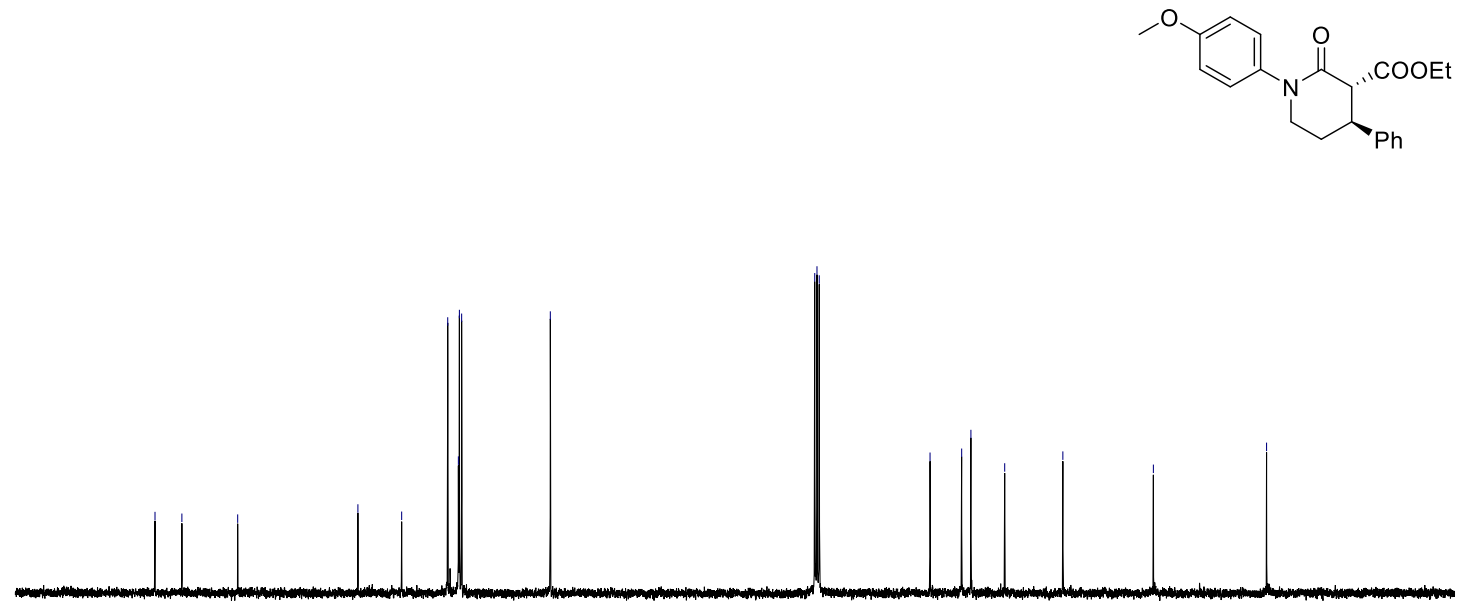

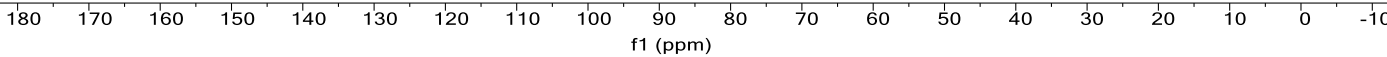




\section{${ }^{1} \mathrm{H}$ NMR (600 MHz, $\left.\mathrm{CDCl}_{3}\right)$ of compound $2 \mathrm{c}$}

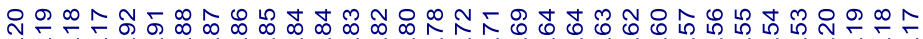
NNN

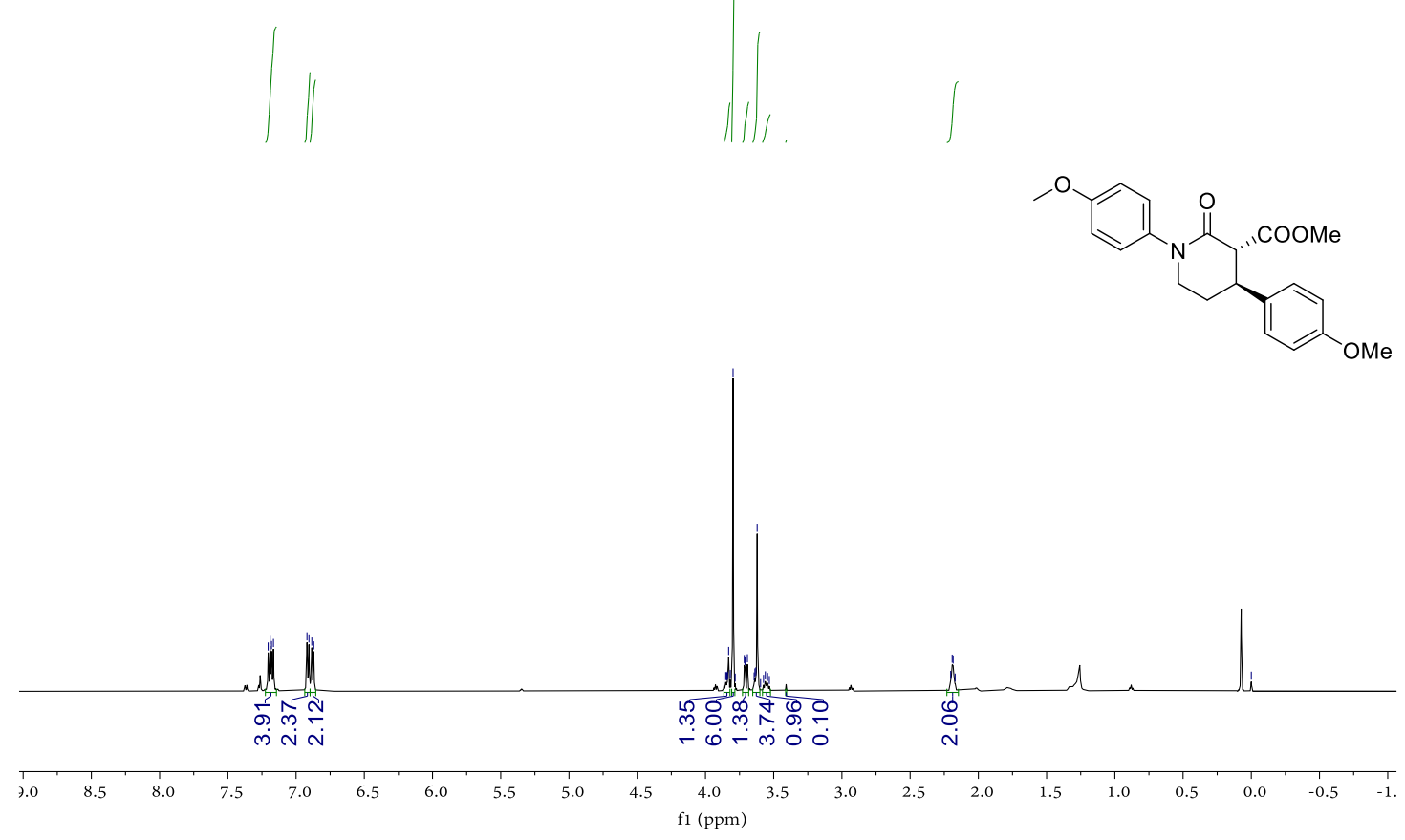

${ }^{13} \mathrm{C}\left\{{ }^{1} \mathrm{H}\right\}$ NMR (151 MHz, $\left.\mathrm{CDCl}_{3}\right)$ of compound 2c

赵

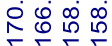

กู่

岗

$\stackrel{\cos }{\cos }$

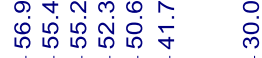

114

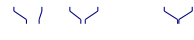

पit ।

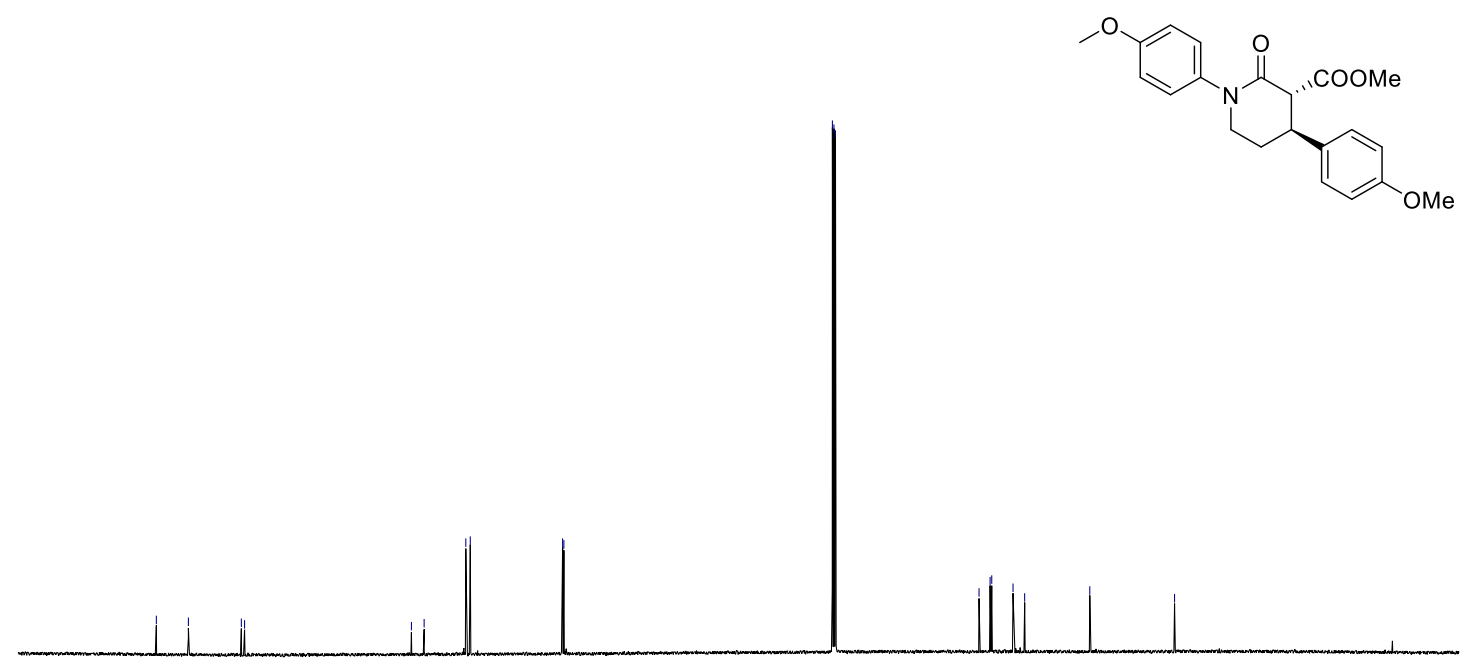

$\begin{array}{llll}180 & 170 & 160 & 150\end{array}$

13

$120 \quad 110 \quad 100$ 


\section{${ }^{1} \mathrm{H}$ NMR (400 MHz, $\mathrm{CDCl}_{3}$ ) of compound 2d}

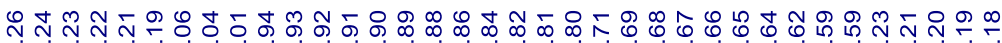

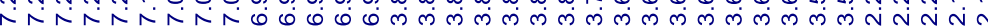

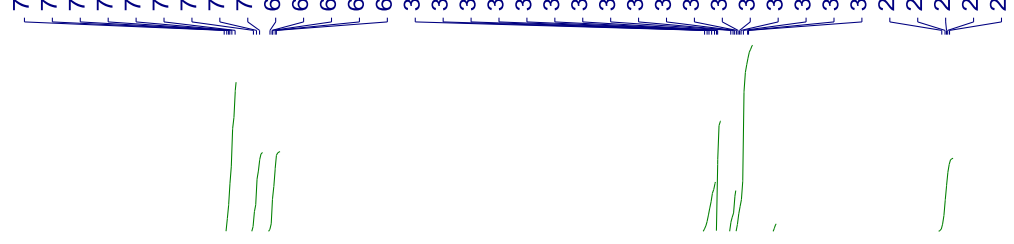

$\stackrel{\circ}{\circ}$
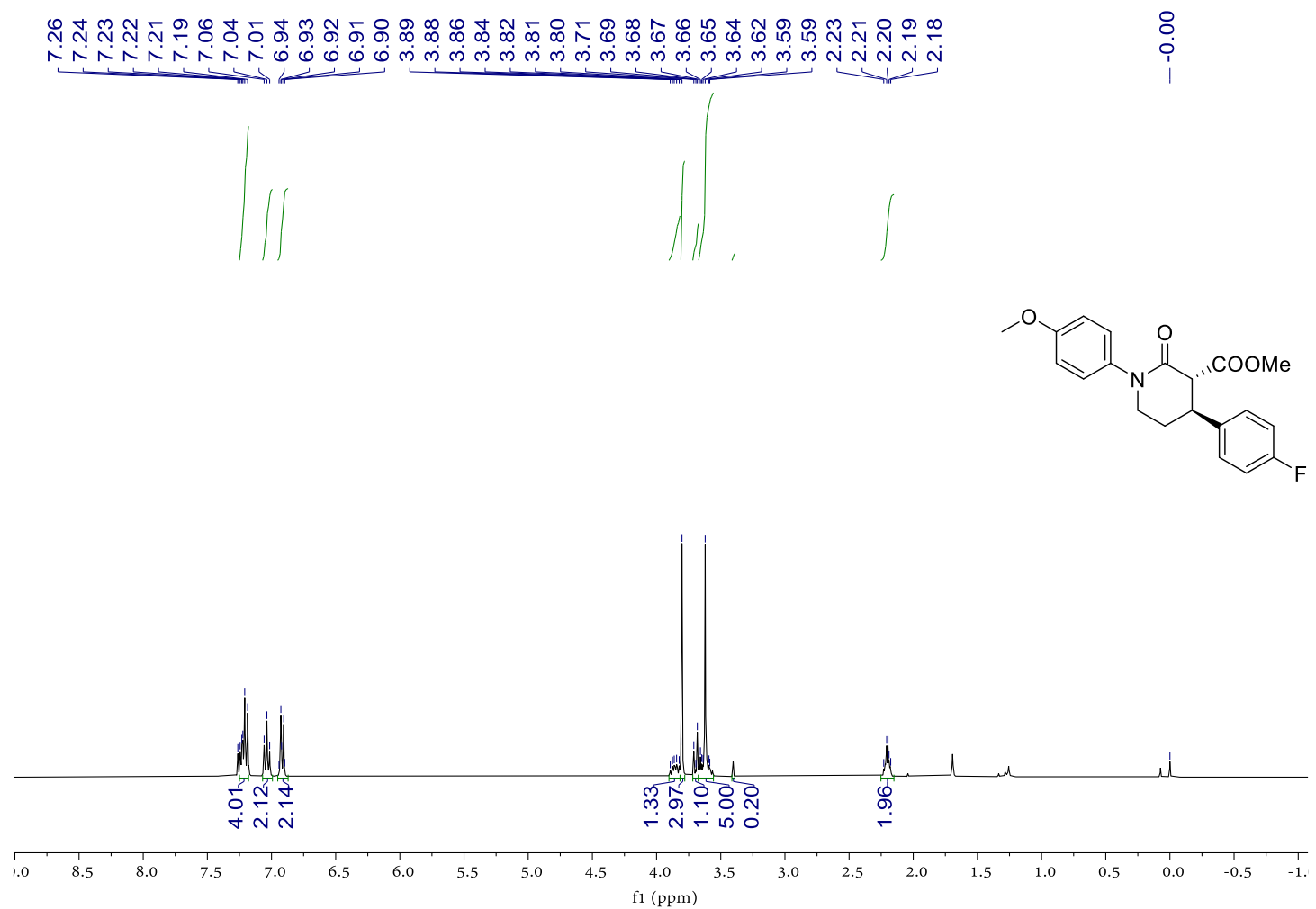

${ }^{19}$ F NMR (376 MHz, $\left.\mathrm{CDCl}_{3}\right)$ of compound 2d
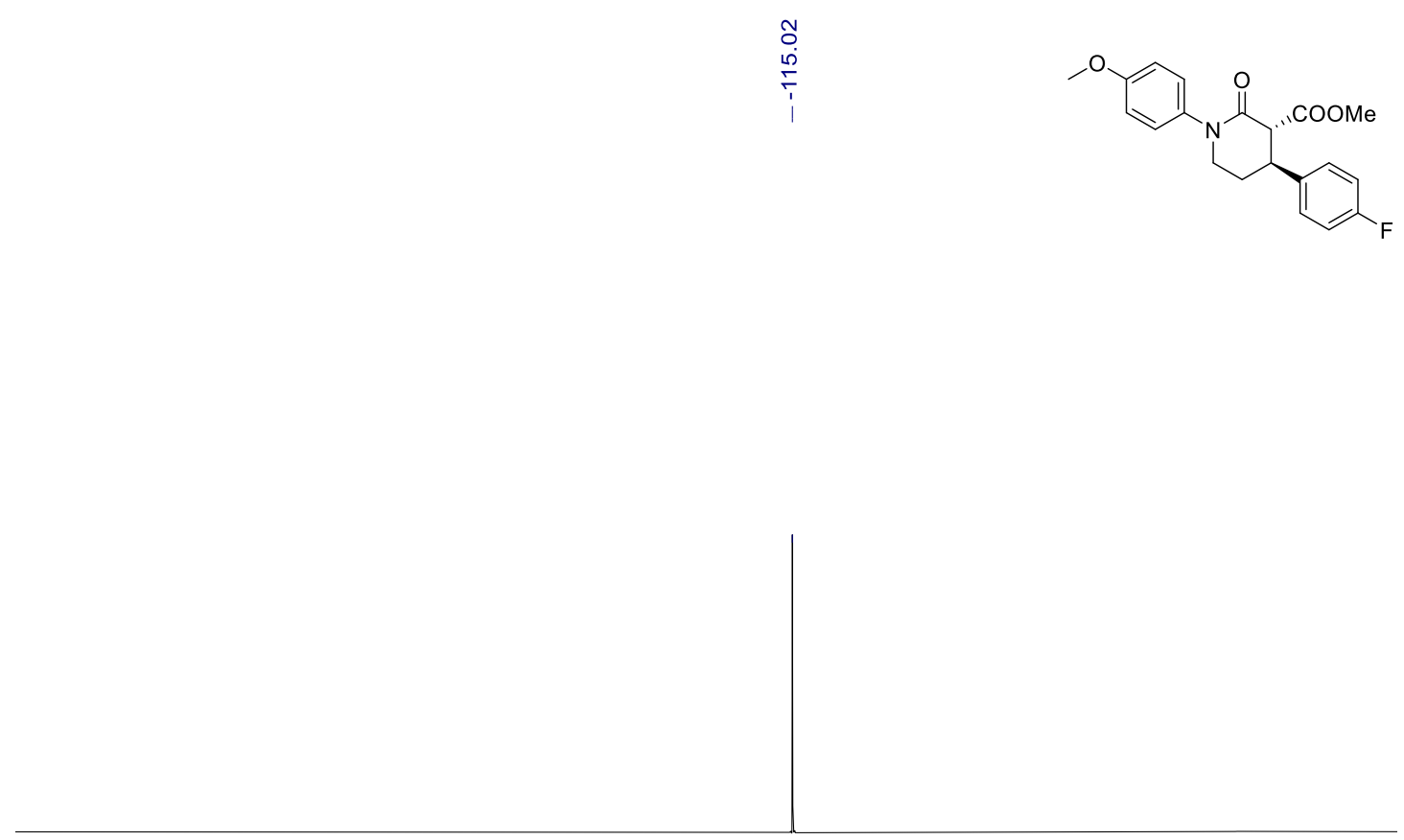

$\begin{array}{lllllllllllllllllllllllllllllllll}20 & 10 & 0 & -10 & -20 & -30 & -40 & -50 & -60 & -70 & -80 & -90 & -100 & -110 & -120 & -130 & -140 & -150 & -160 & -170 & -180 & -190 & -200 & -210 & -2\end{array}$ $\mathrm{f}_{1}(\mathrm{ppm})$ 


\section{${ }^{13} \mathrm{C}\left\{{ }^{1} \mathrm{H}\right\}$ NMR (151 MHz, $\left.\mathrm{CDCl}_{3}\right)$ of compound 2d}

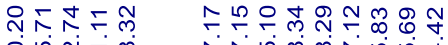

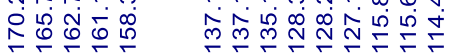

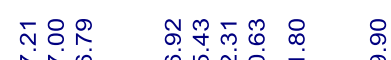

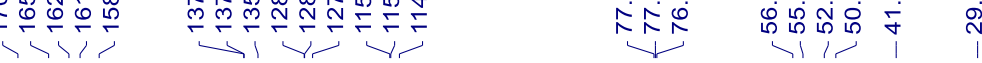

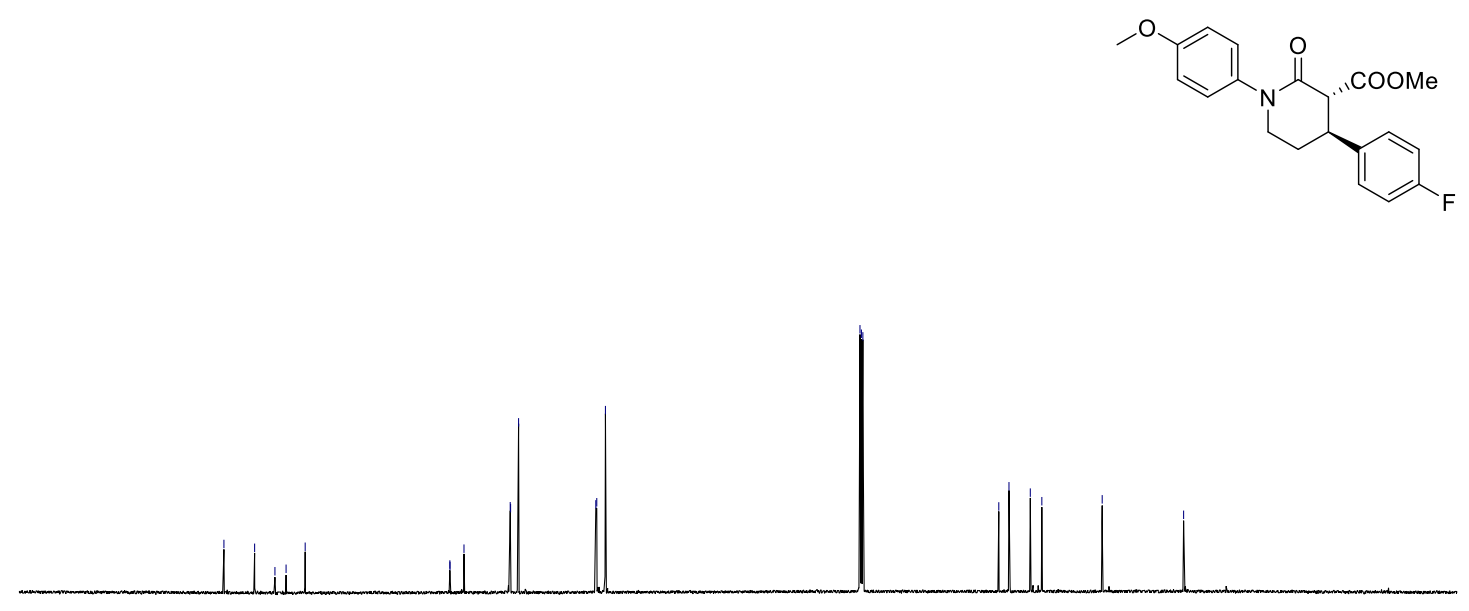

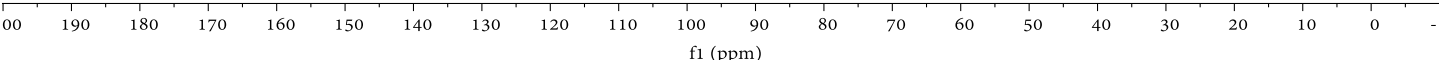




\section{${ }^{1} \mathrm{H}$ NMR (400 MHz, $\mathrm{CDCl}_{3}$ ) of compound $2 \mathrm{e}$}

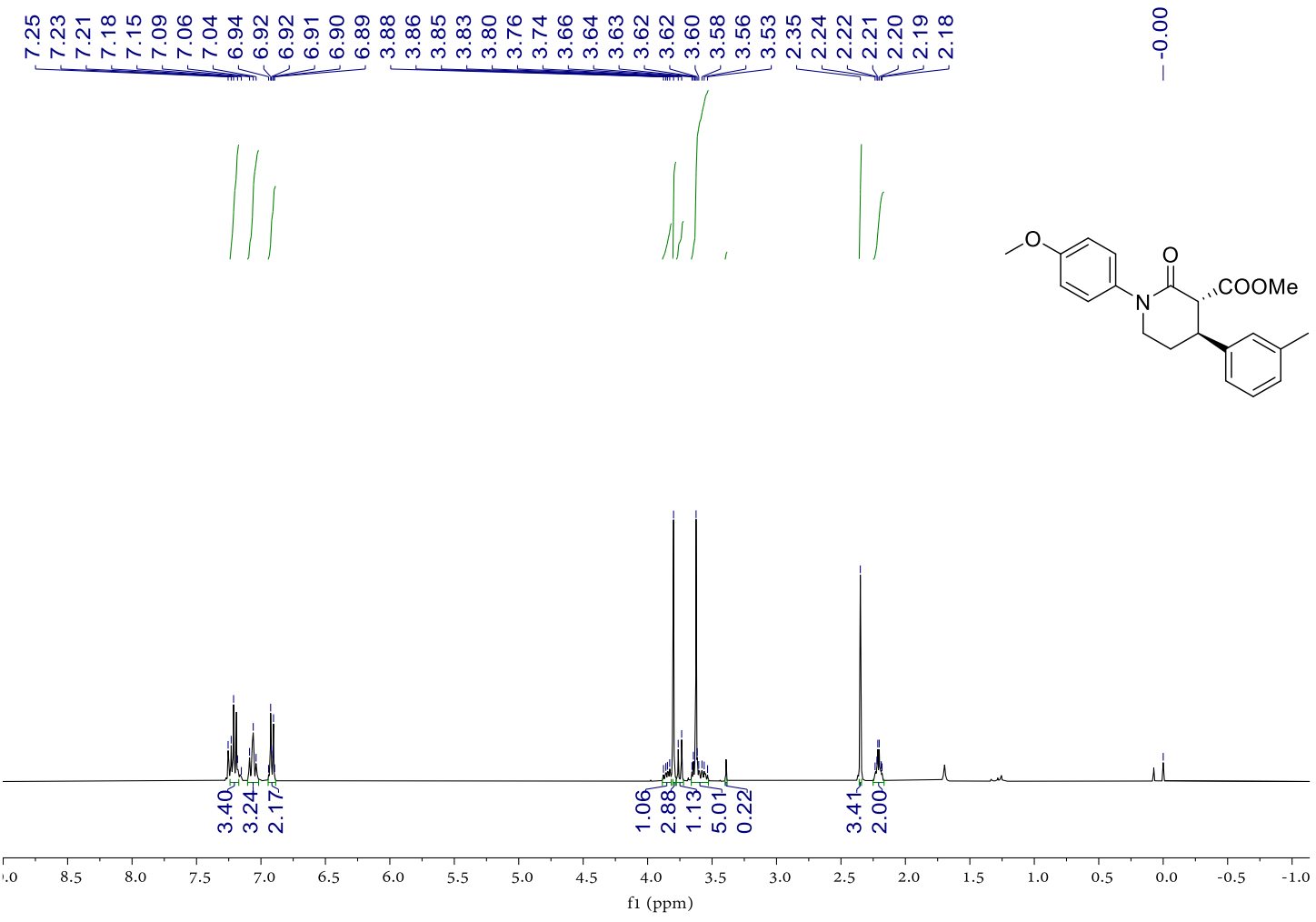

${ }^{13} \mathrm{C}\left\{{ }^{1} \mathrm{H}\right\}$ NMR (101 MHz, $\left.\mathrm{CDCl}_{3}\right)$ of compound 2e

\begin{tabular}{|c|c|c|}
\hline 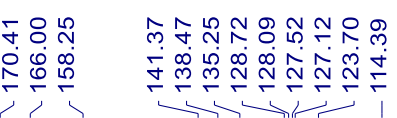 & 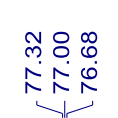 & 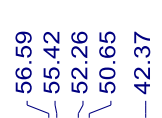 \\
\hline
\end{tabular}
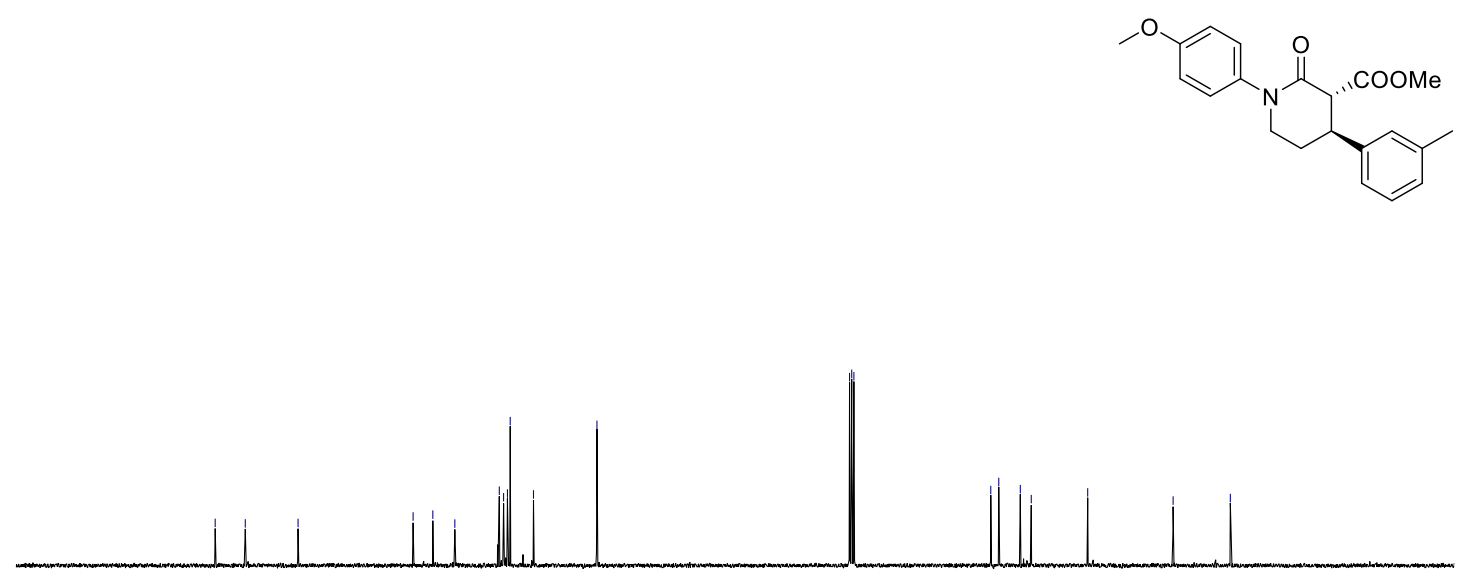
${ }^{1} \mathrm{H}$ NMR (600 MHz, $\left.\mathrm{CDCl}_{3}\right)$ of compound $2 \mathrm{f}$

ํํำ

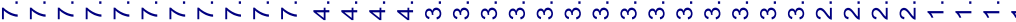

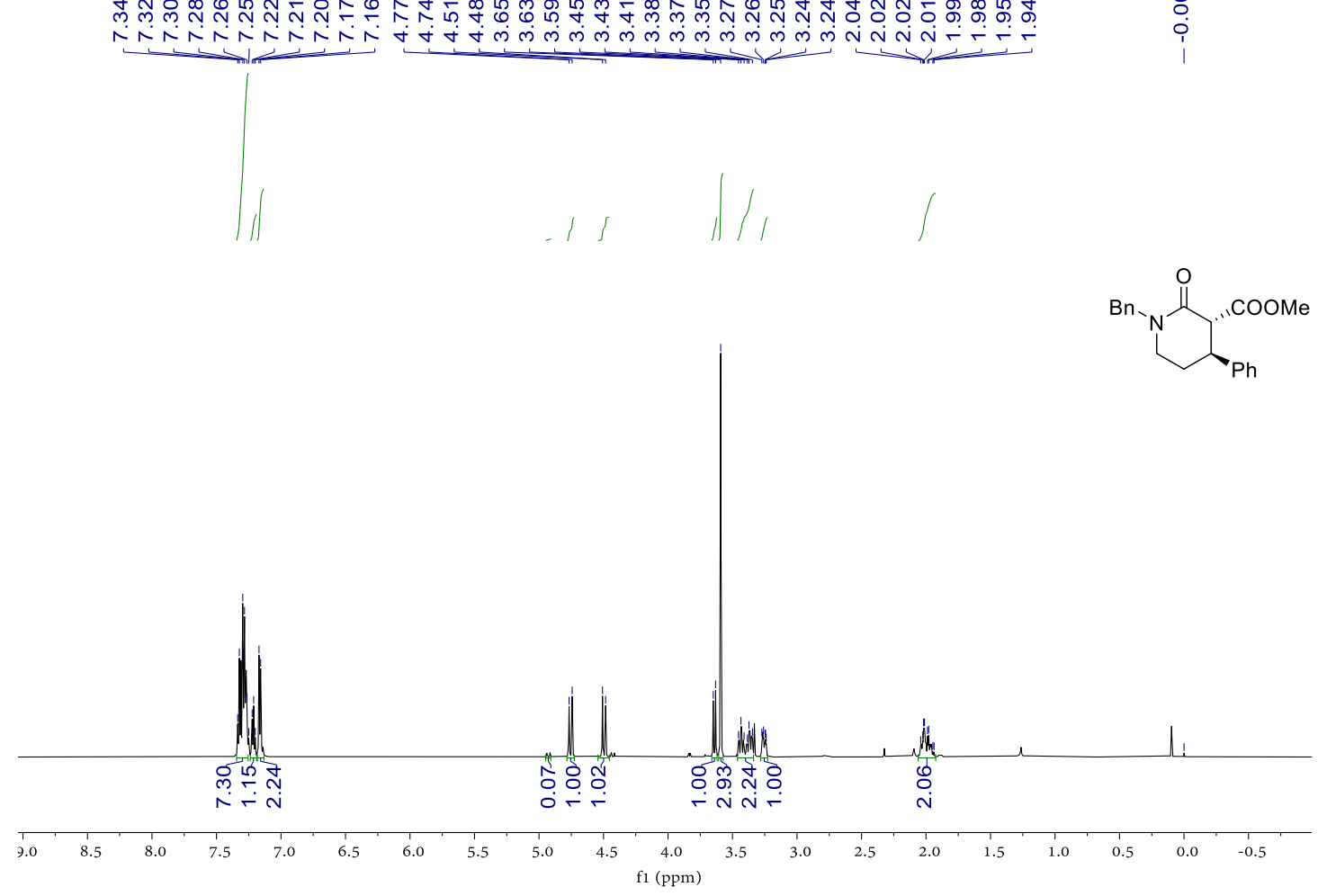

${ }^{13} \mathrm{C}\left\{{ }^{1} \mathrm{H}\right\}$ NMR (151 MHz, $\left.\mathrm{CDCl}_{3}\right)$ of compound $2 \mathrm{f}$

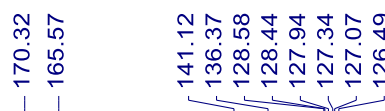

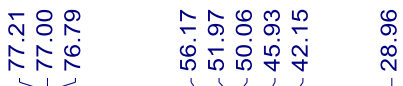
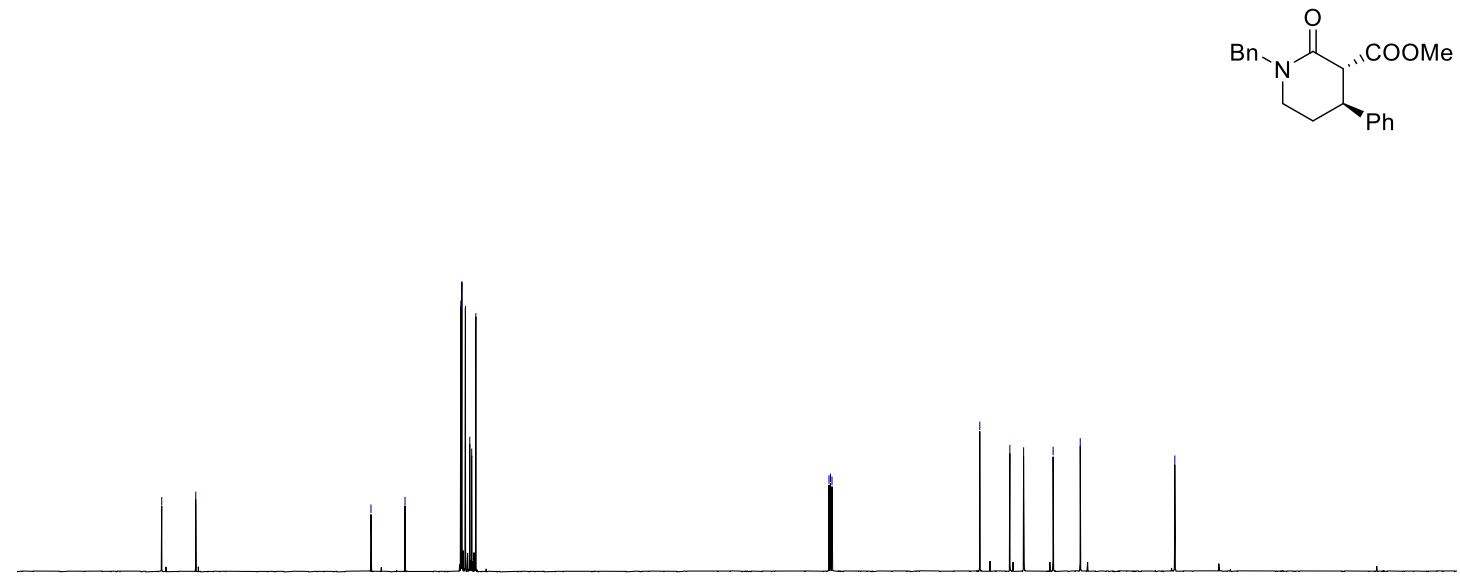

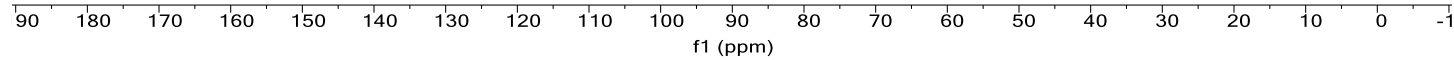


${ }^{1} \mathrm{H}$ NMR (400 MHz, $\left.\mathrm{CDCl}_{3}\right)$ of compound $2 \mathrm{~g}$

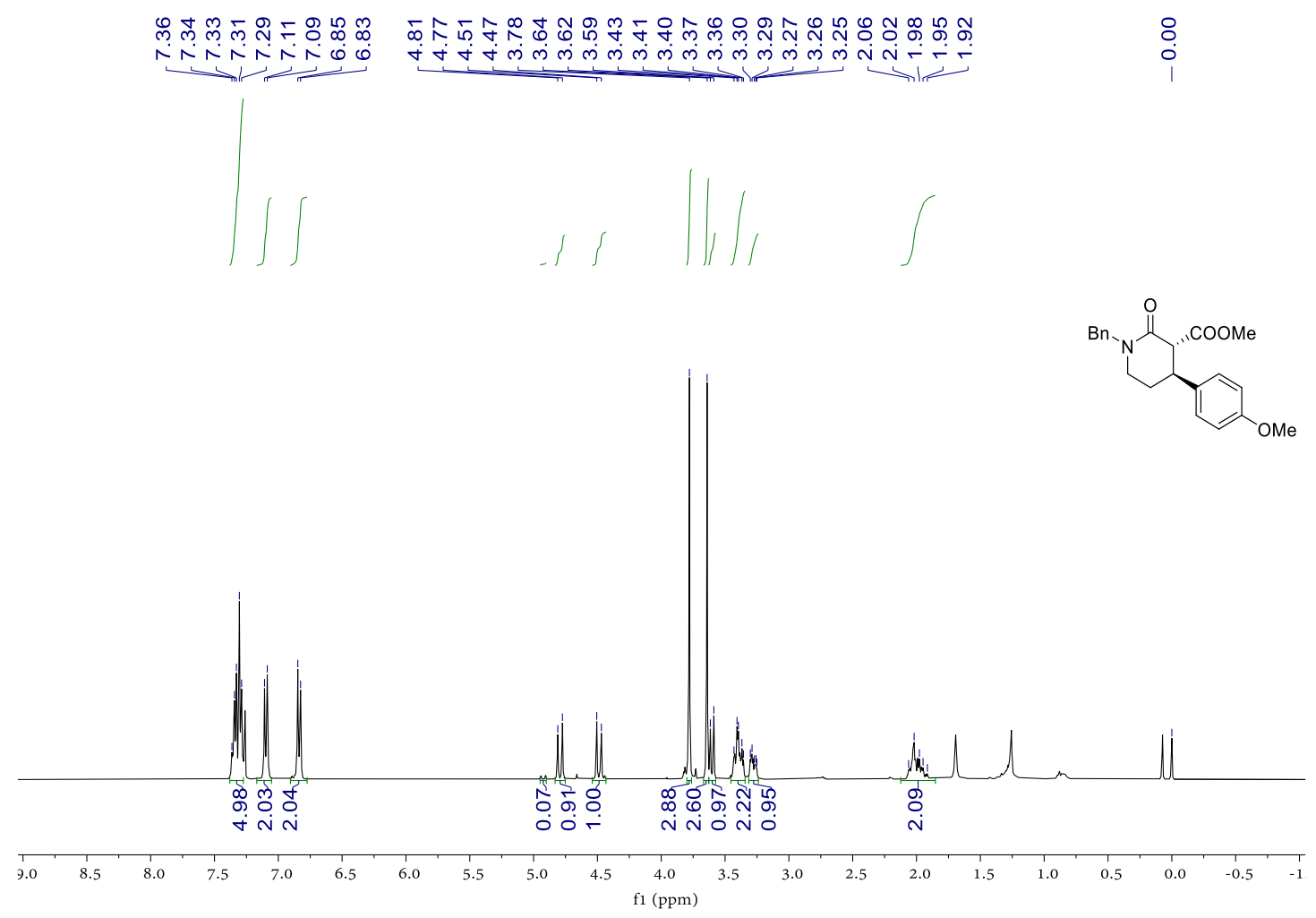

${ }^{13} \mathrm{C}\left\{{ }^{1} \mathrm{H}\right\}$ NMR (101 MHz, $\left.\mathrm{CDCl}_{3}\right)$ of compound $2 \mathrm{~g}$

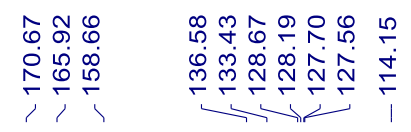

బ్లం

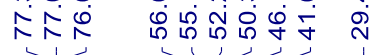
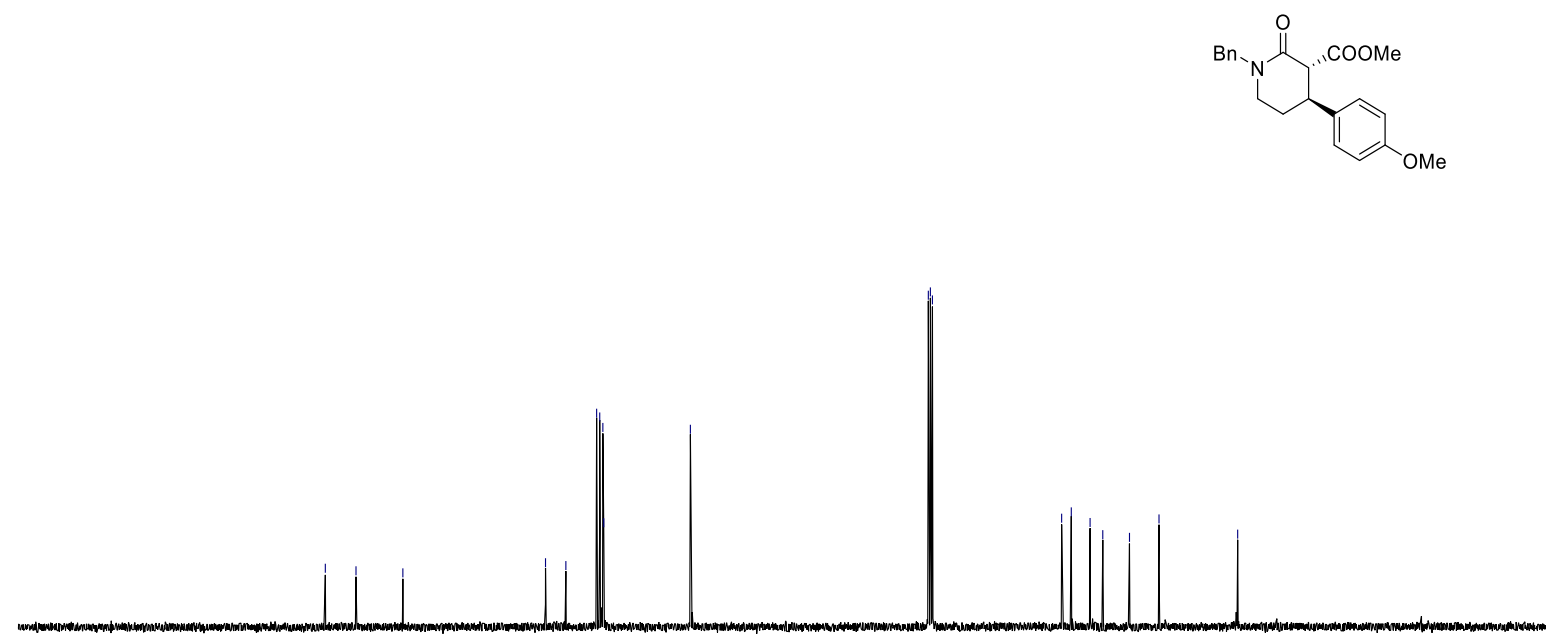

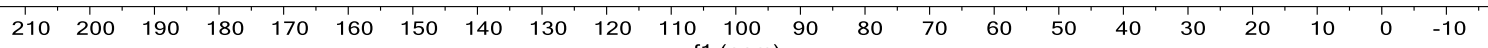


${ }^{1} \mathrm{H}$ NMR (400 MHz, $\mathrm{CDCl}_{3}$ ) of compound $2 \mathrm{~h}$

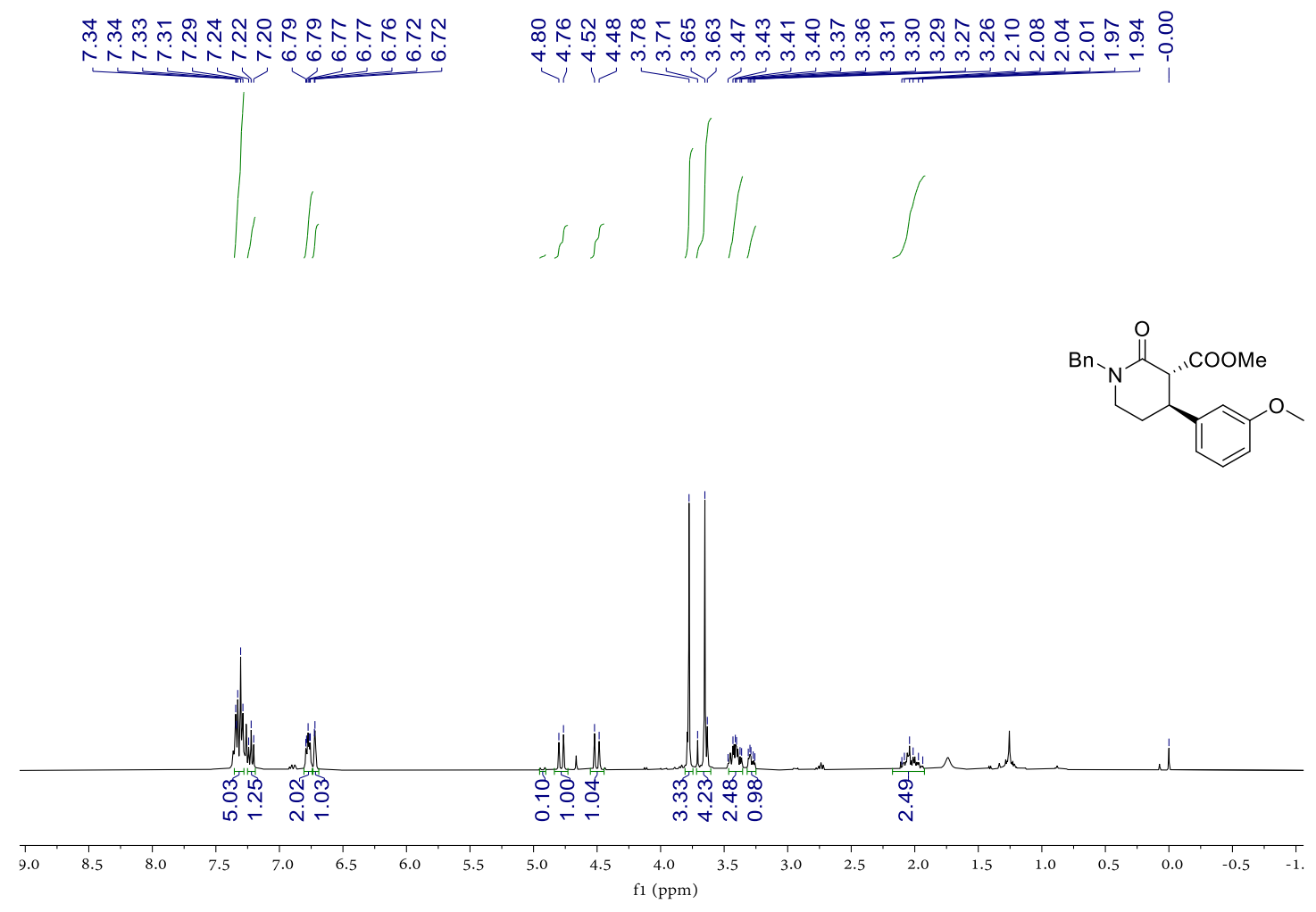

${ }^{13} \mathrm{C}\left\{{ }^{1} \mathrm{H}\right\}$ NMR (151 MHz, $\left.\mathrm{CDCl}_{3}\right)$ of compound $2 \mathrm{~h}$

โ็

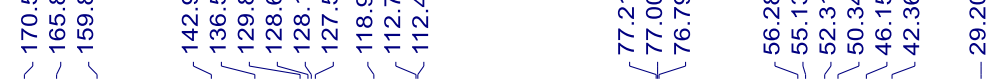

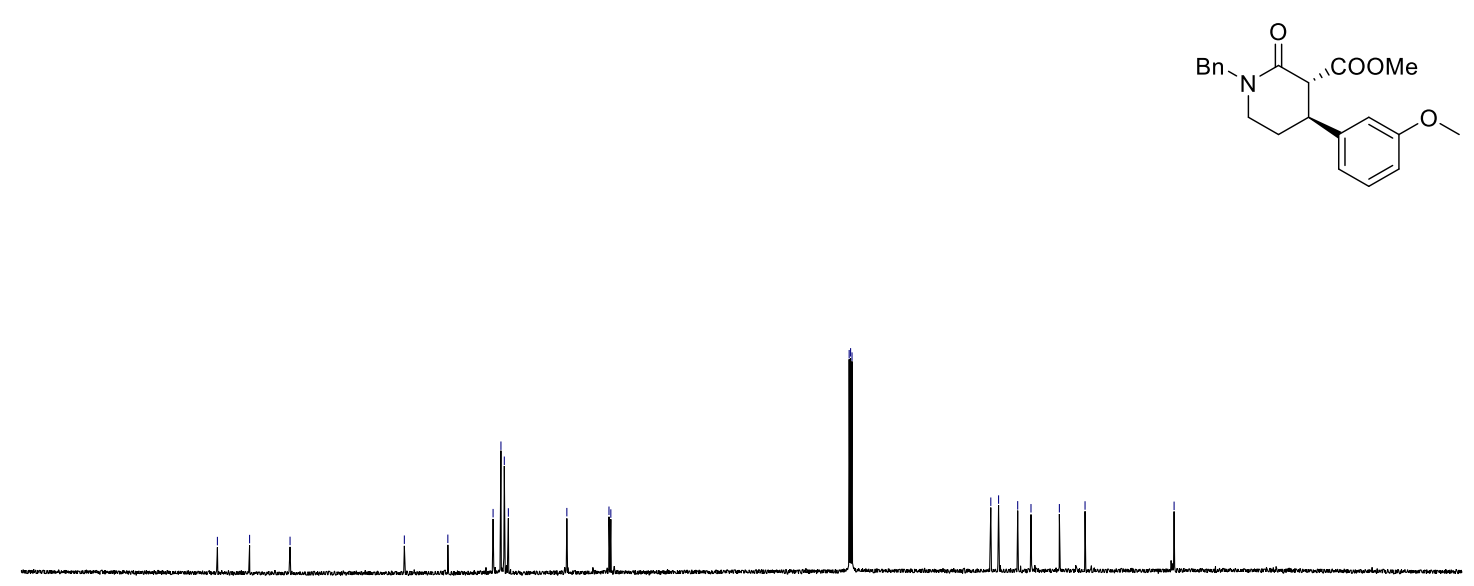

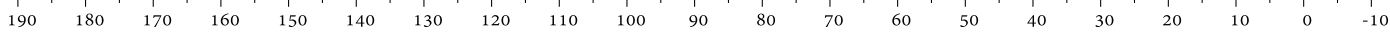


${ }^{1} \mathrm{H}$ NMR (400 MHz, $\left.\mathrm{CDCl}_{3}\right)$ of compound $2 \mathrm{i}$

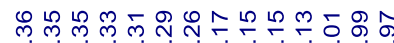

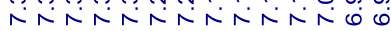

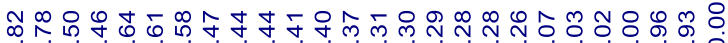
ن
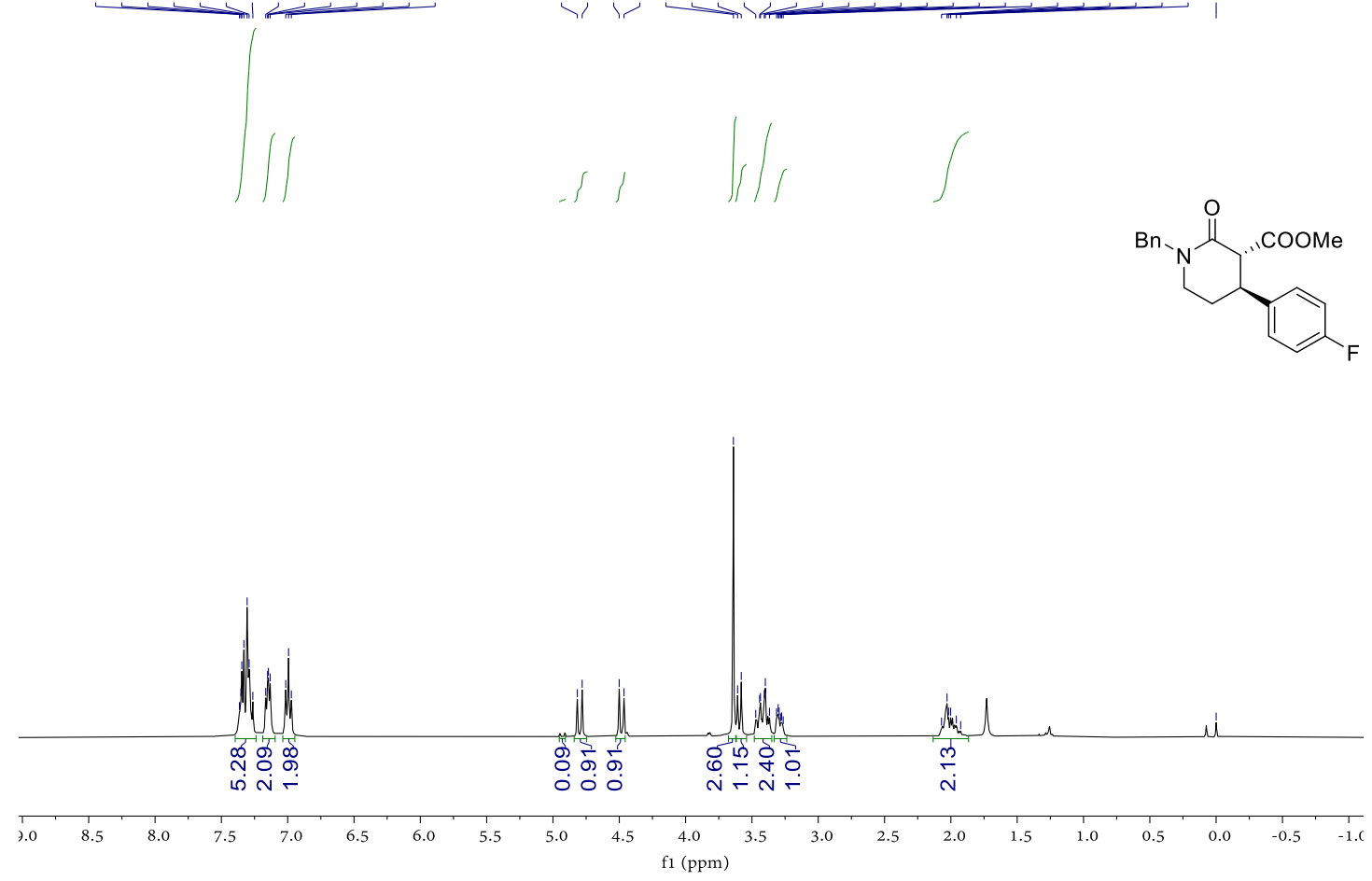

${ }^{19}$ F NMR (376 MHz, $\mathrm{CDCl}_{3}$ ) of compound $2 \mathrm{i}$
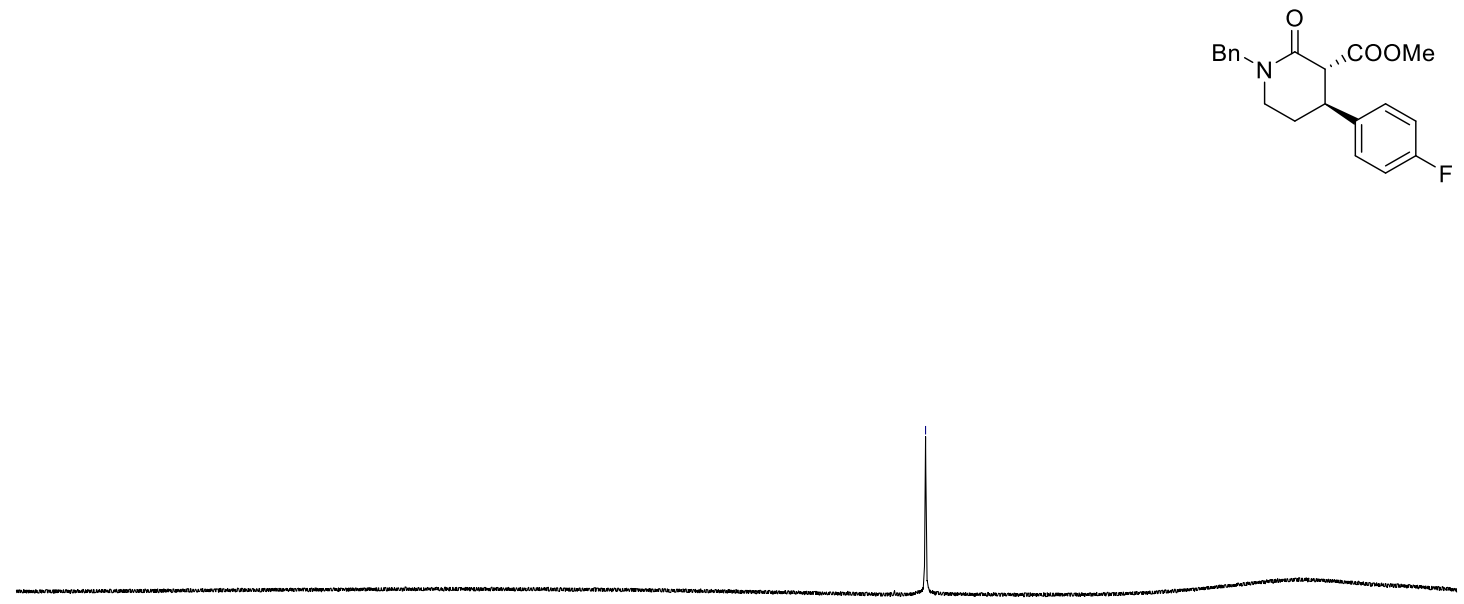

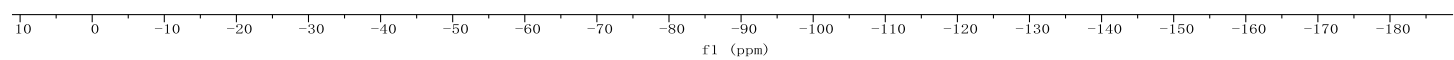


${ }^{13} \mathrm{C}\left\{{ }^{1} \mathrm{H}\right\}$ NMR (101 MHz, $\left.\mathrm{CDCl}_{3}\right)$ of compound $2 \mathrm{i}$

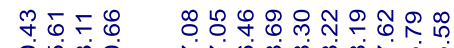

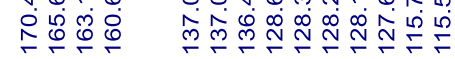

잉

令点

윰쑈용요

ڤึ่

$\bar{m}$

ก
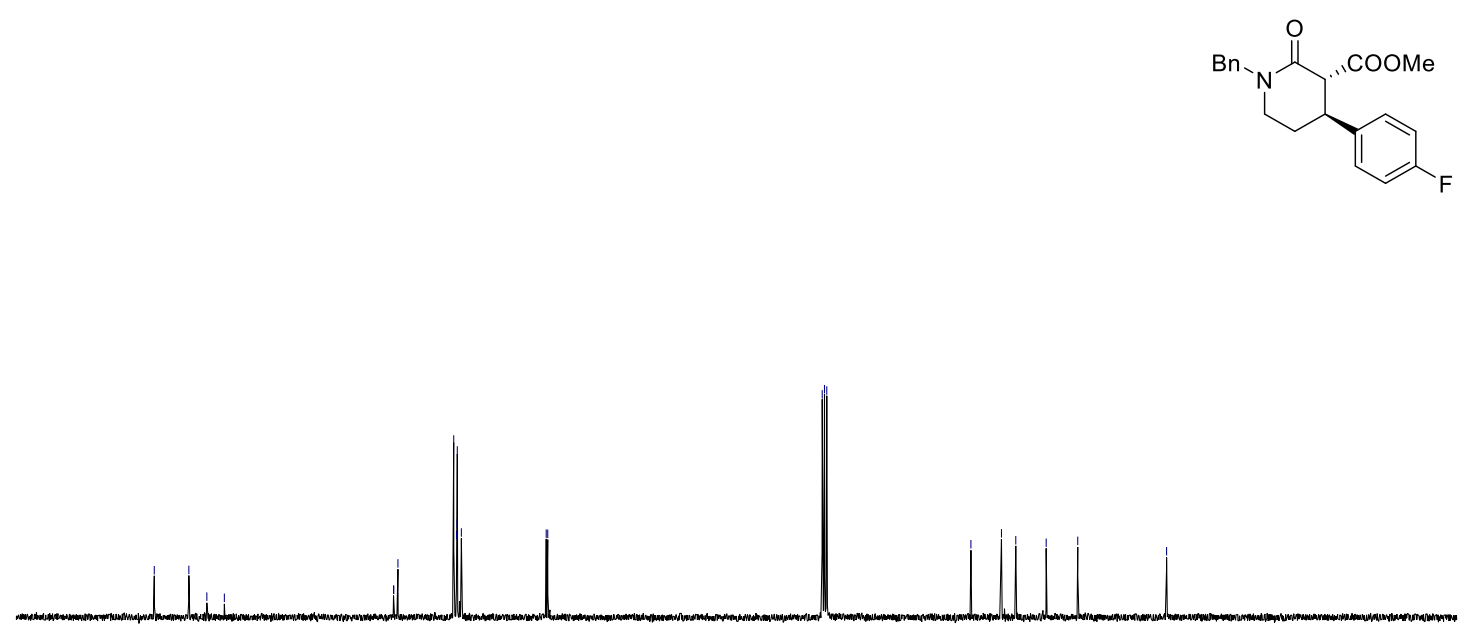

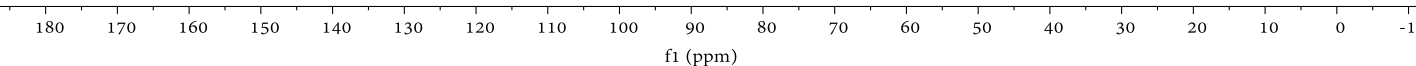




\section{${ }^{1} \mathrm{H}$ NMR (400 MHz, $\mathrm{CDCl}_{3}$ ) of compound $2 \mathrm{j}$}

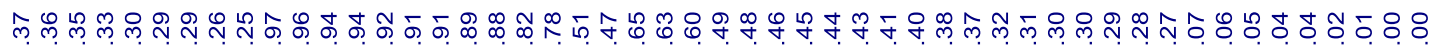

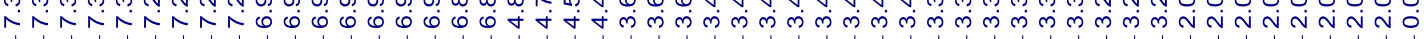

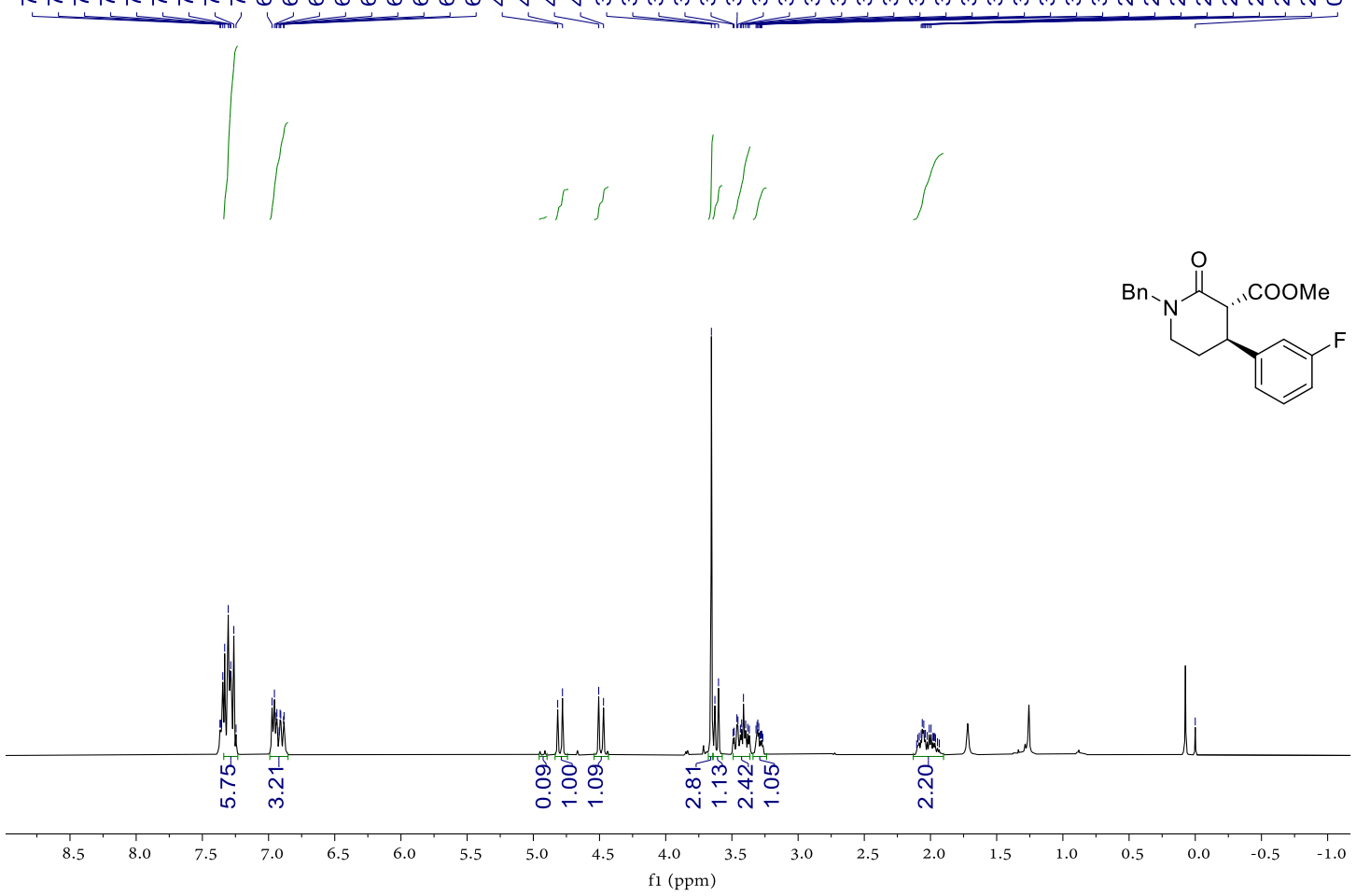

${ }^{19}$ F NMR (376 MHz, $\left.\mathrm{CDCl}_{3}\right)$ of compound $2 \mathrm{j}$
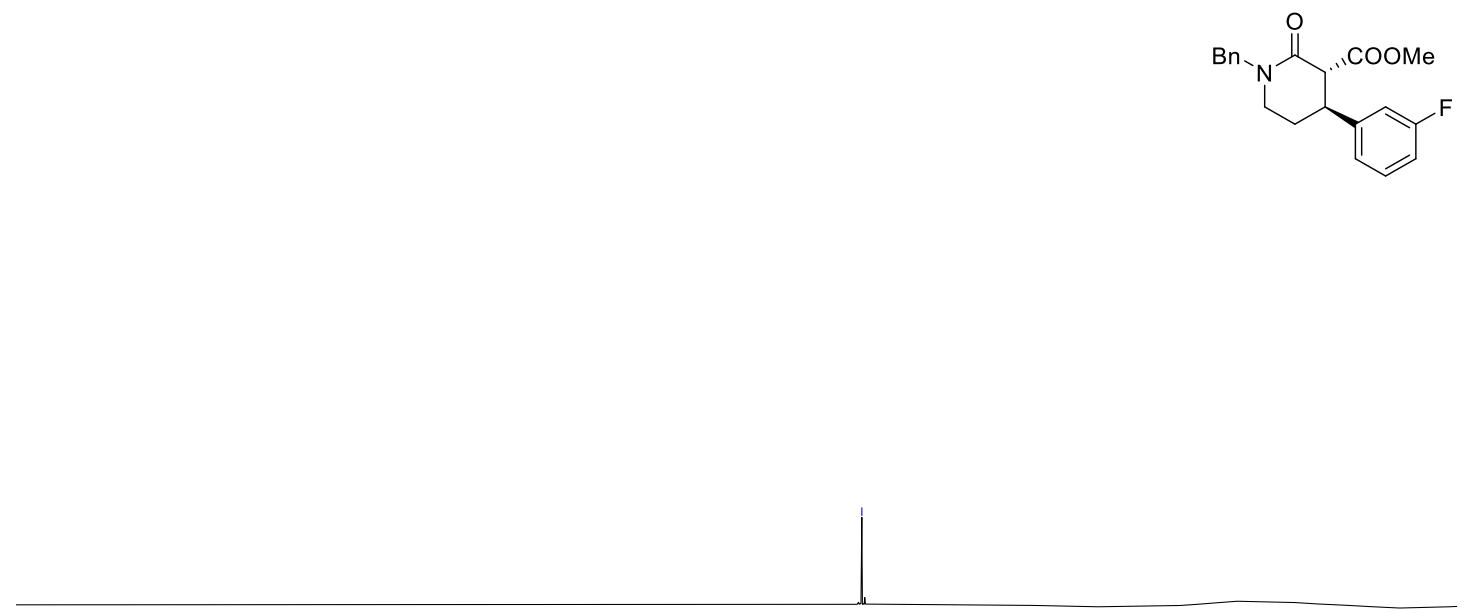
${ }^{13} \mathrm{C}\left\{{ }^{1} \mathrm{H}\right\}$ NMR (151 MHz, $\left.\mathrm{CDCl}_{3}\right)$ of compound $2 \mathrm{j}$

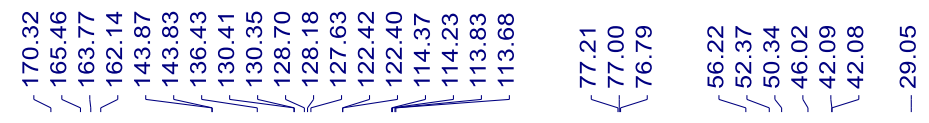
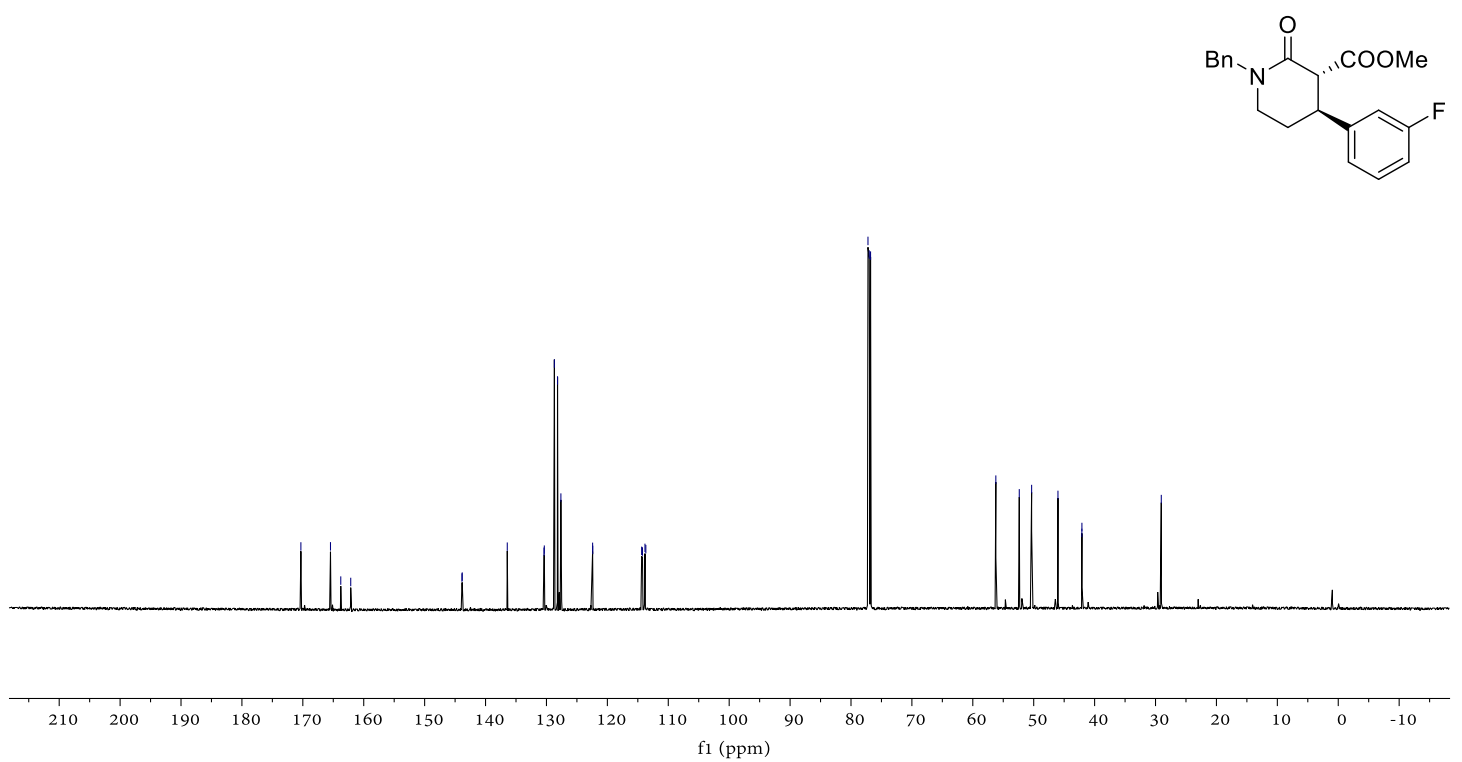

S58 
${ }^{1} \mathrm{H}$ NMR (400 MHz, $\mathrm{CDCl}_{3}$ ) of compound $2 \mathrm{k}$

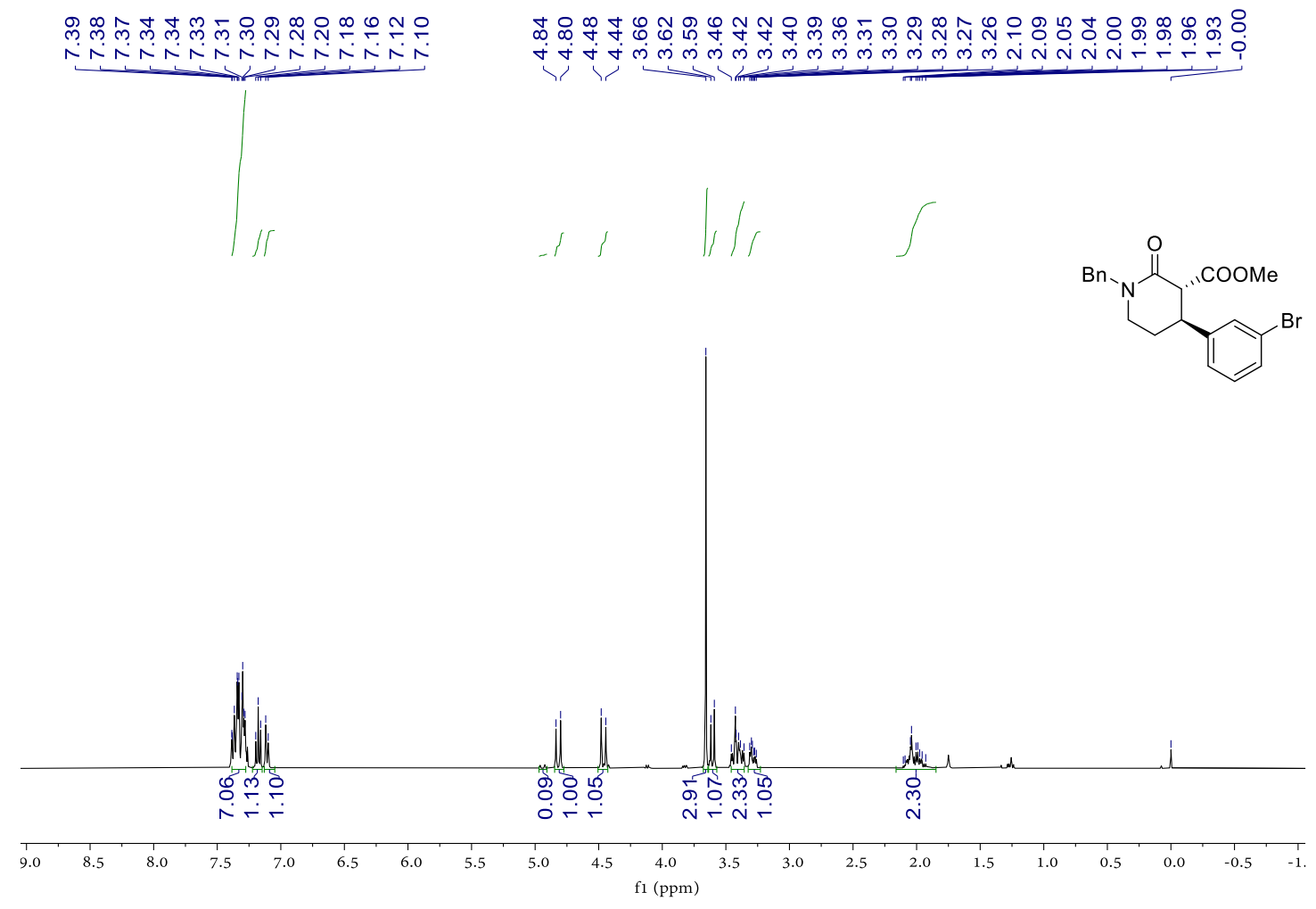

${ }^{13} \mathrm{C}\left\{{ }^{1} \mathrm{H}\right\}$ NMR (101 MHz, $\left.\mathrm{CDCl}_{3}\right)$ of compound $2 \mathrm{k}$

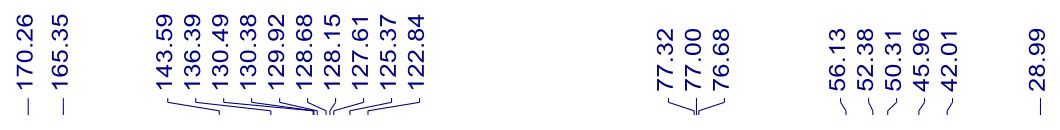

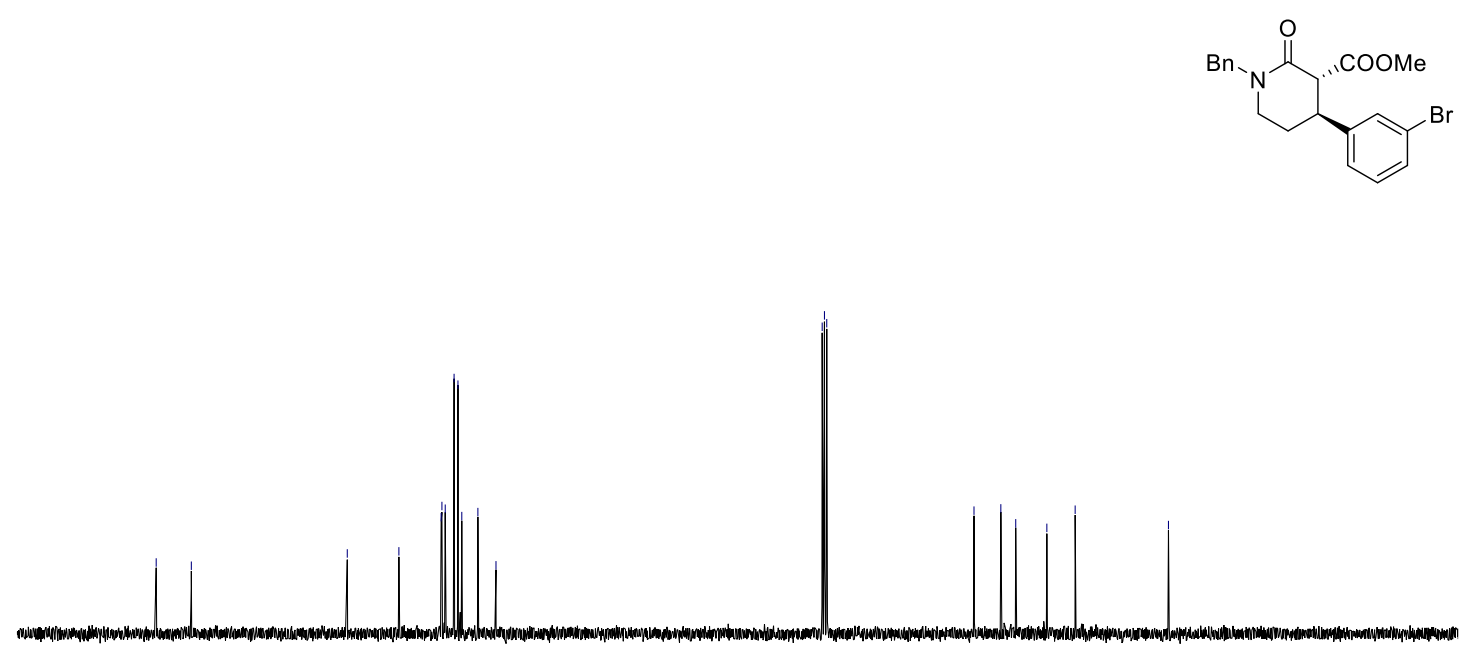

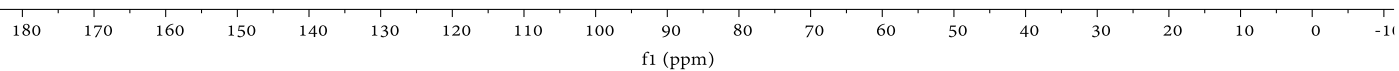


${ }^{1} \mathrm{H}$ NMR (400 MHz, $\left.\mathrm{CDCl}_{3}\right)$ of compound $2 \mathrm{I}$

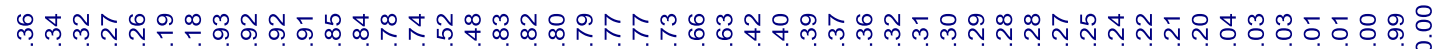

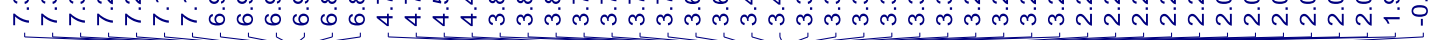

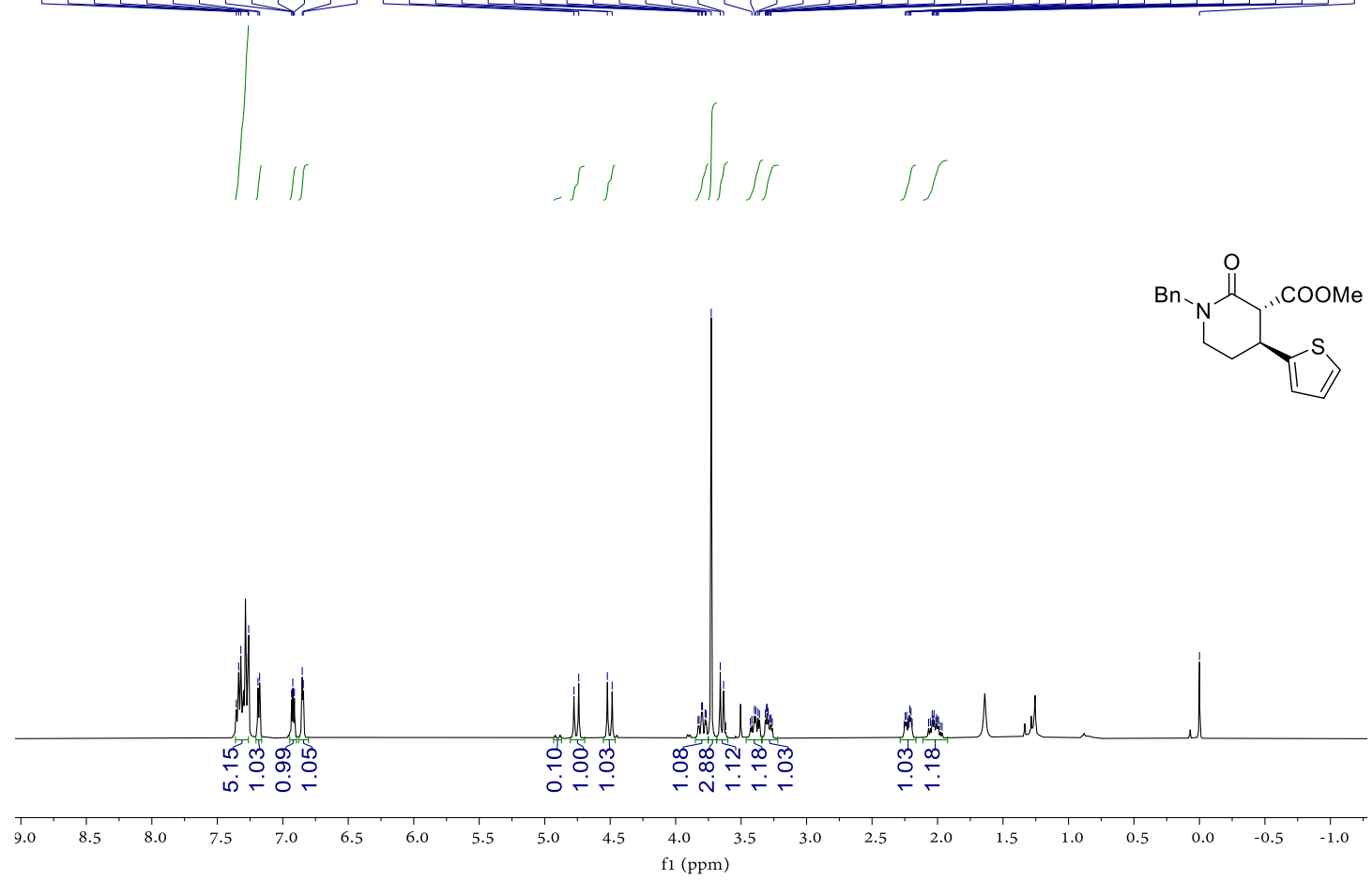

${ }^{13} \mathrm{C}\left\{{ }^{1} \mathrm{H}\right\}$ NMR (151 MHz, $\left.\mathrm{CDCl}_{3}\right)$ of compound 21

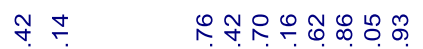

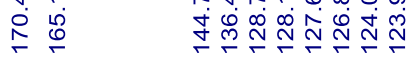

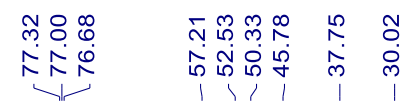

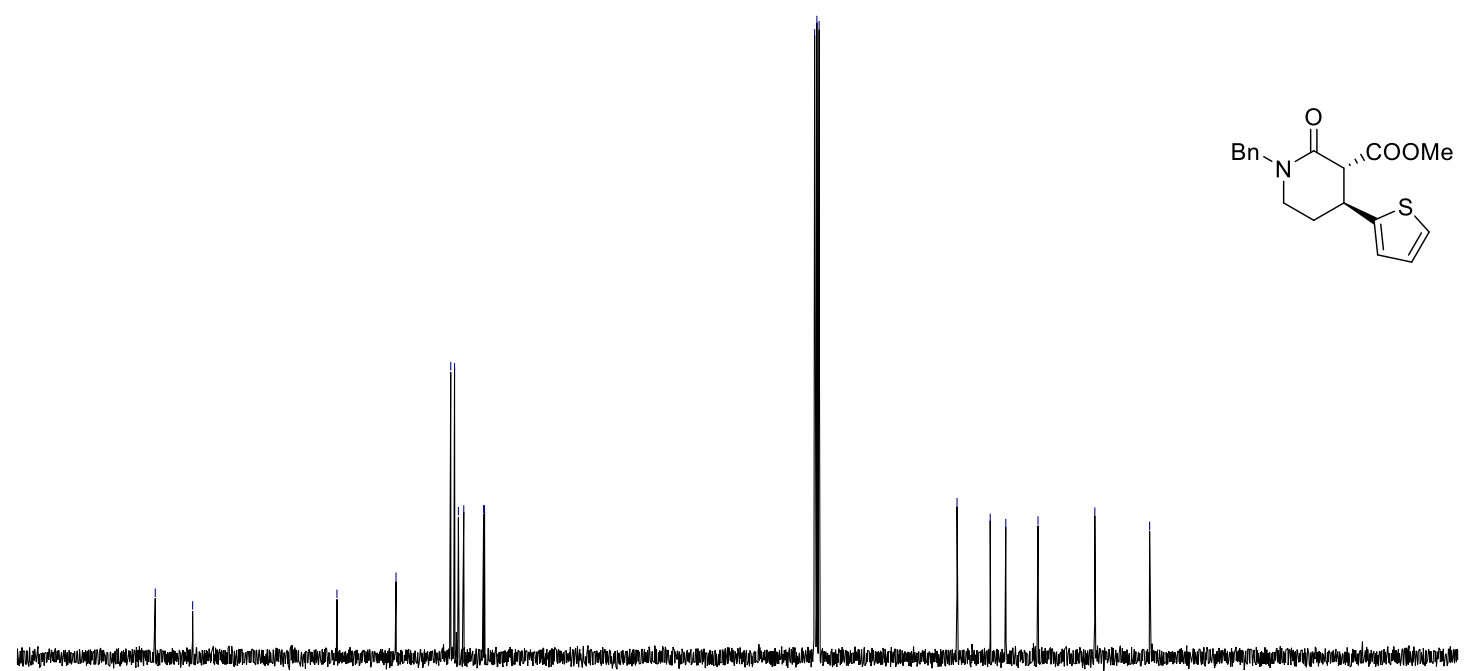

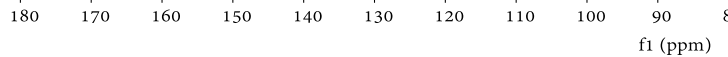




\section{${ }^{1} \mathrm{H}$ NMR (400 MHz, $\mathrm{CDCl}_{3}$ ) of compound $2 \mathrm{~m}$}

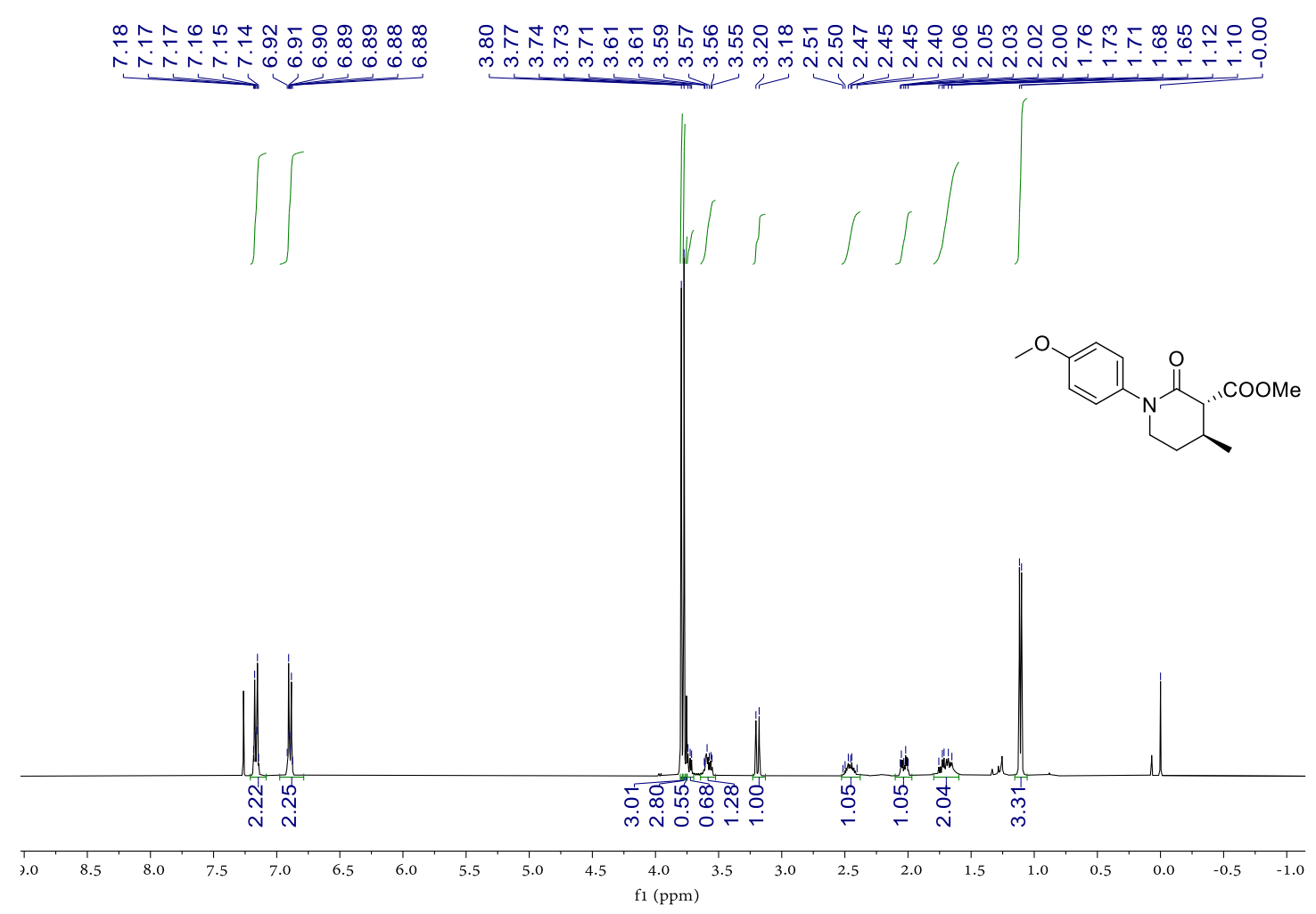

${ }^{13} \mathrm{C}\left\{{ }^{1} \mathrm{H}\right\}$ NMR (101 MHz, $\left.\mathrm{CDCl}_{3}\right)$ of compound $2 \mathrm{~m}$

\begin{tabular}{|c|c|c|c|c|c|c|c|}
\hline 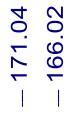 & $\begin{array}{l}\stackrel{\bigcirc}{N} \\
\infty \\
\stackrel{\infty}{\infty} \\
i\end{array}$ & $\begin{array}{l}\stackrel{\rho}{m} \\
\stackrel{\rho}{\rho} \\
\stackrel{m}{1}\end{array}$ & 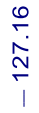 & $\begin{array}{l}\hat{n} \\
\stackrel{+}{+}\end{array}$ & 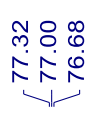 & 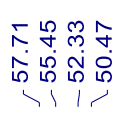 & $\begin{array}{l}+0 \\
+0 \\
\dot{m} 0 \\
1\end{array}$ \\
\hline
\end{tabular}

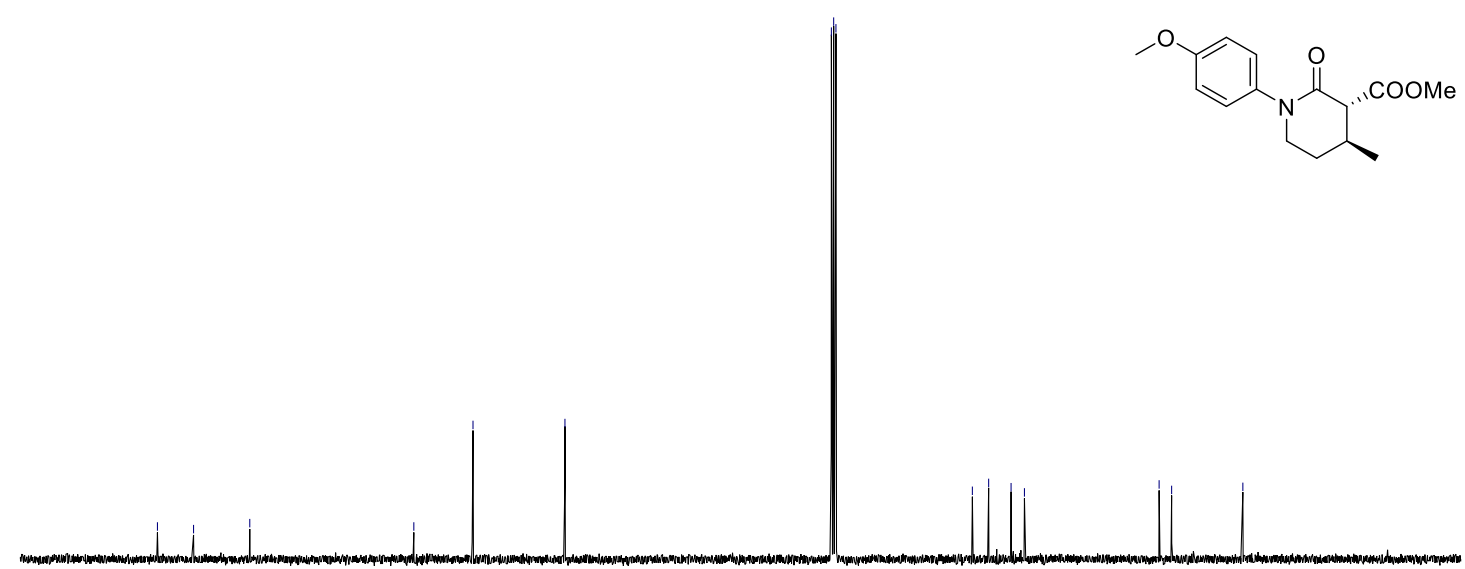




\section{${ }^{1} \mathrm{H}$ NMR (600 MHz, $\mathrm{CDCl}_{3}$ ) of compound 2n}

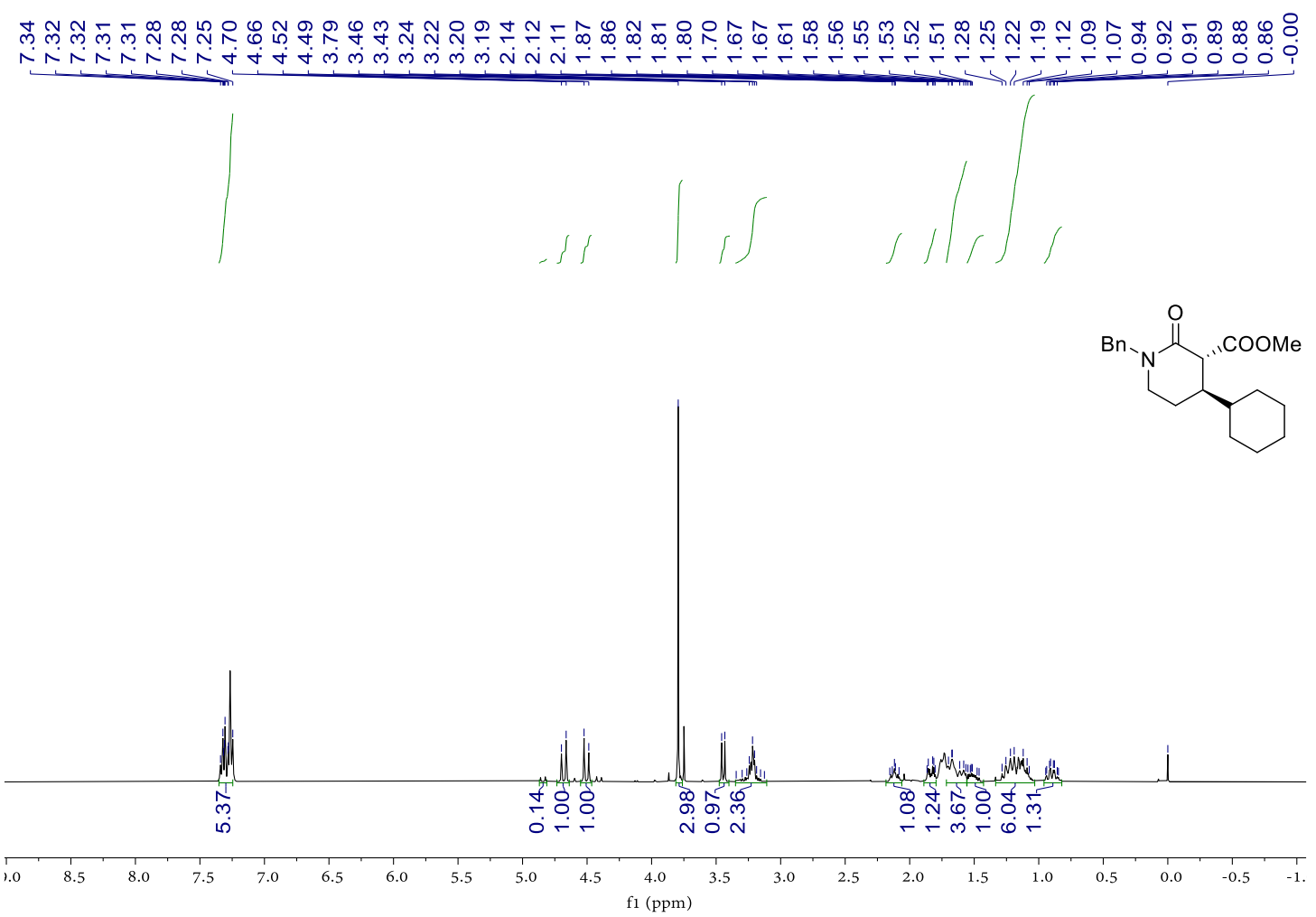

${ }^{13} \mathrm{C}\left\{{ }^{1} \mathrm{H}\right\}$ NMR (151 MHz, $\left.\mathrm{CDCl}_{3}\right)$ of compound 2n

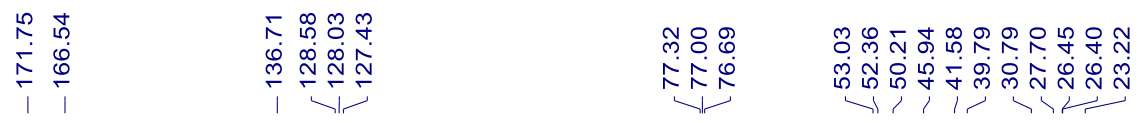
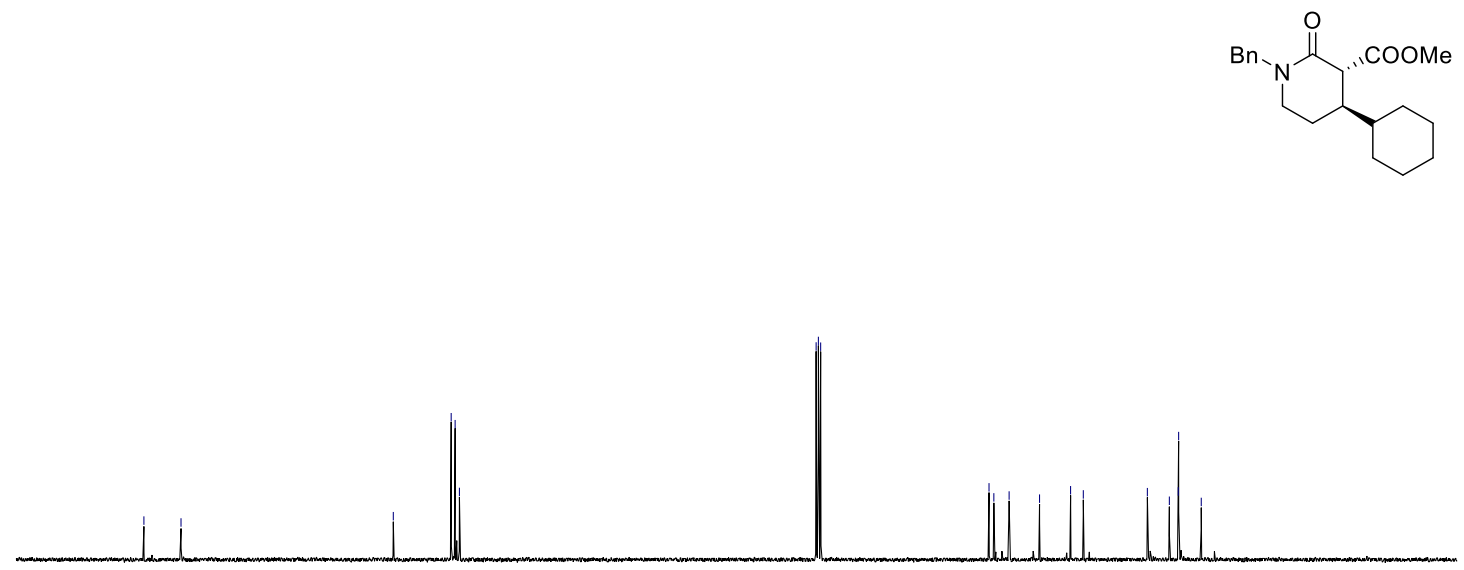
${ }^{1} \mathrm{H}$ NMR (600 MHz, $\mathrm{CDCl}_{3}$ ) of compound 20

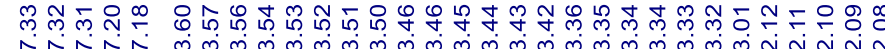

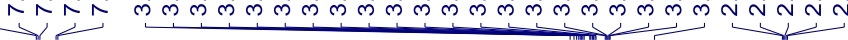
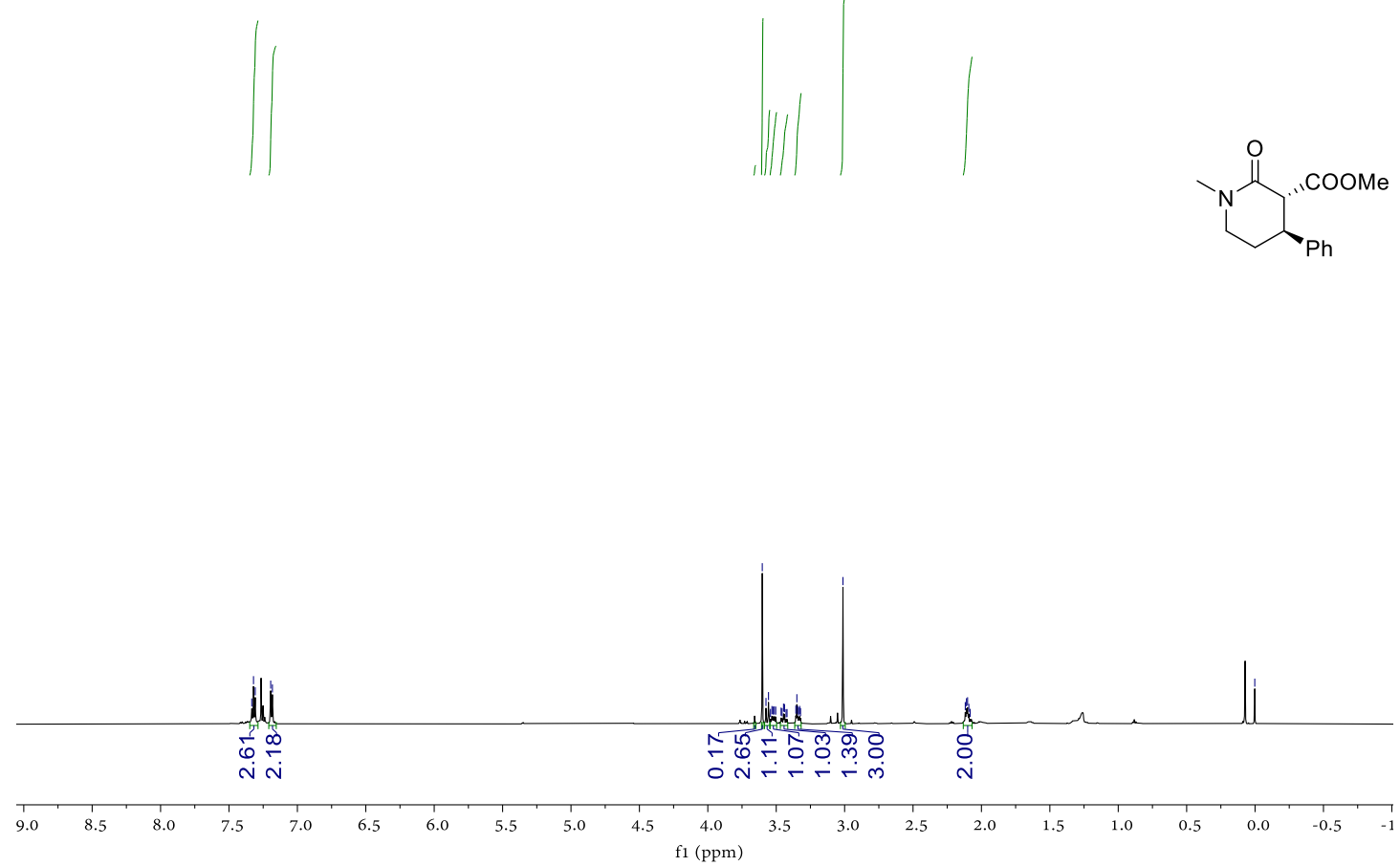

${ }^{13} \mathrm{C}\left\{{ }^{1} \mathrm{H}\right\}$ NMR (151 MHz, $\left.\mathrm{CDCl}_{3}\right)$ of compound 20

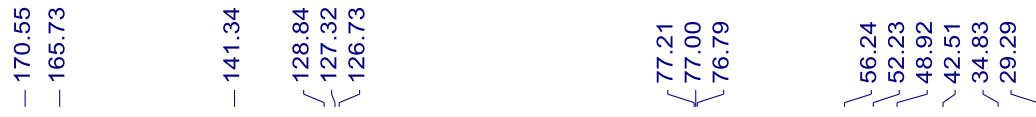

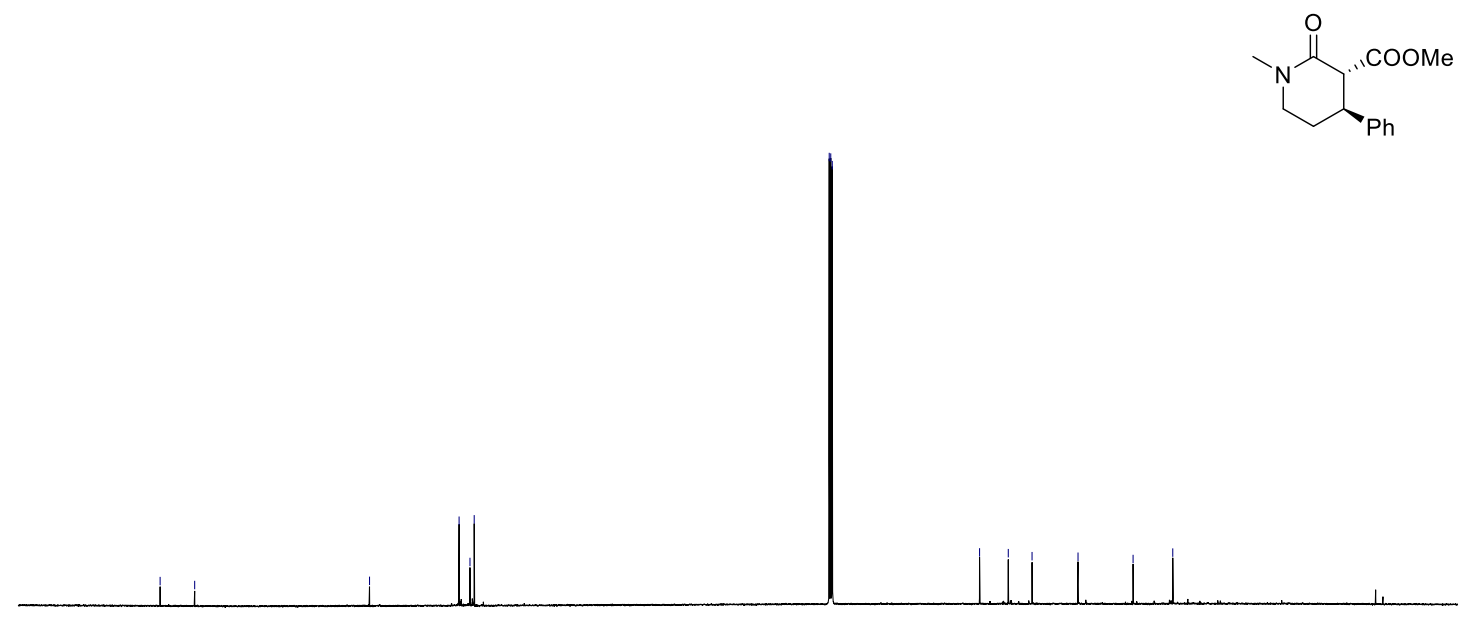

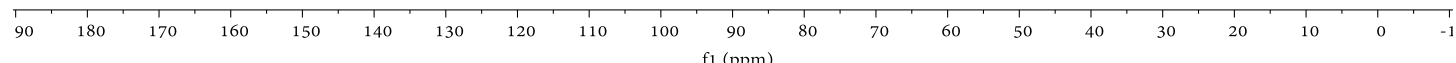




\section{${ }^{1} \mathrm{H}$ NMR (600 MHz, $\mathrm{CDCl}_{3}$ ) of compound 3a}

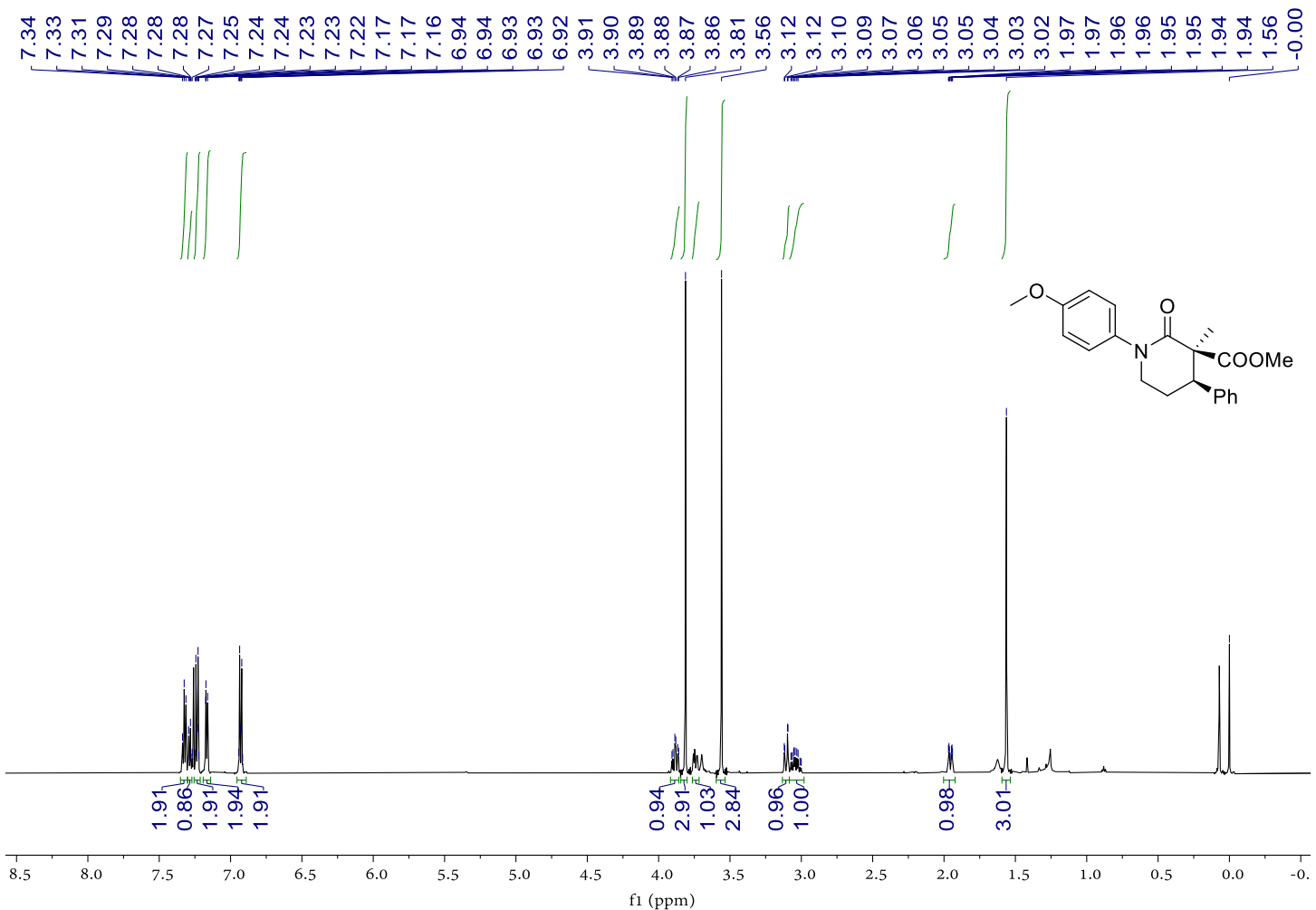

${ }^{13} \mathrm{C}\left\{{ }^{1} \mathrm{H}\right\}$ NMR (151 MHz, $\left.\mathrm{CDCl}_{3}\right)$ of compound 3a

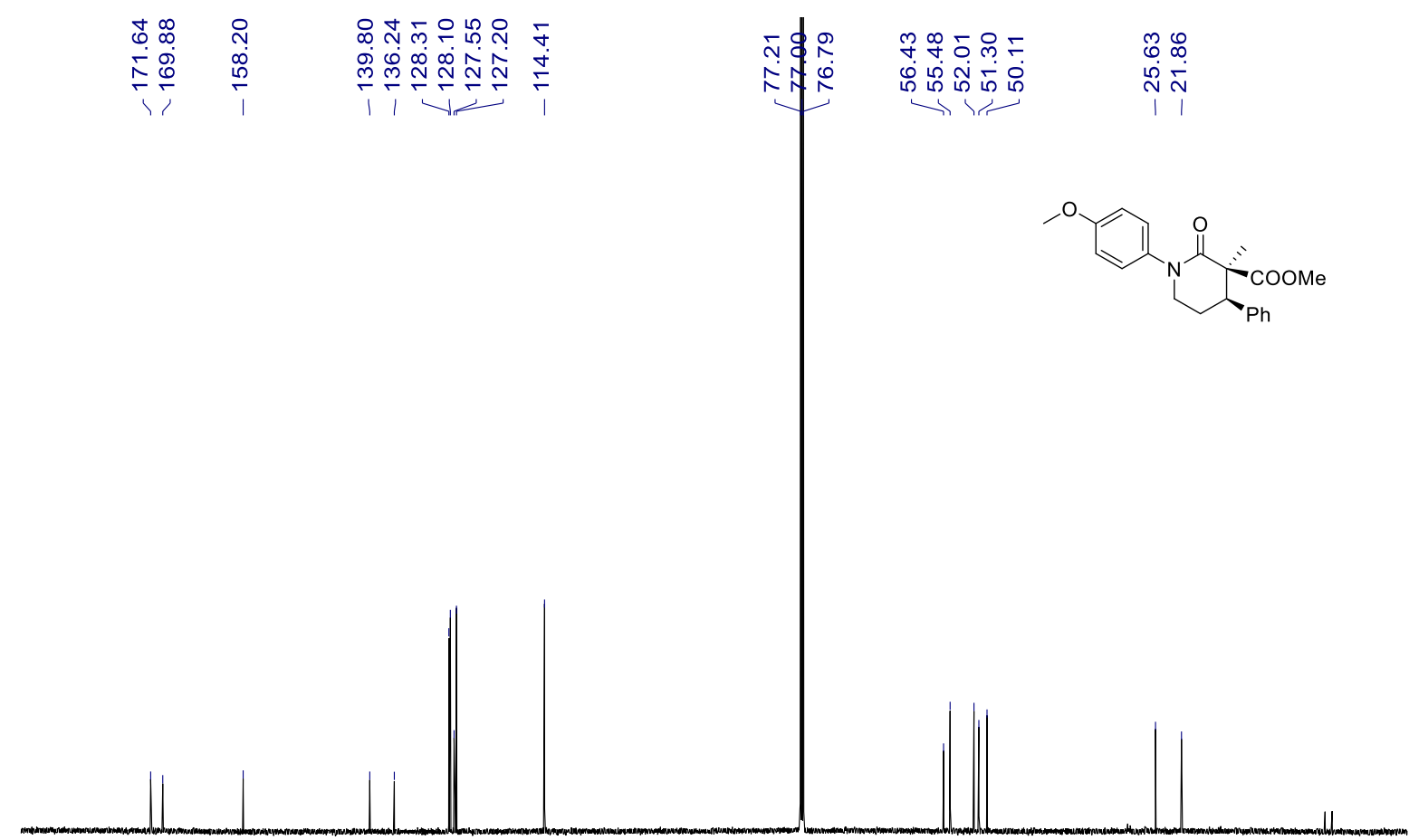

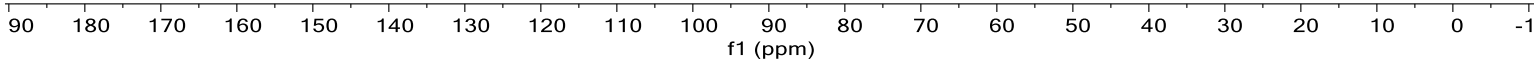




\section{${ }^{1} \mathrm{H}$ NMR (600 MHz, $\mathrm{CDCl}_{3}$ ) of compound 3b}

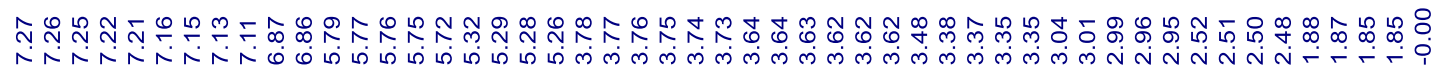
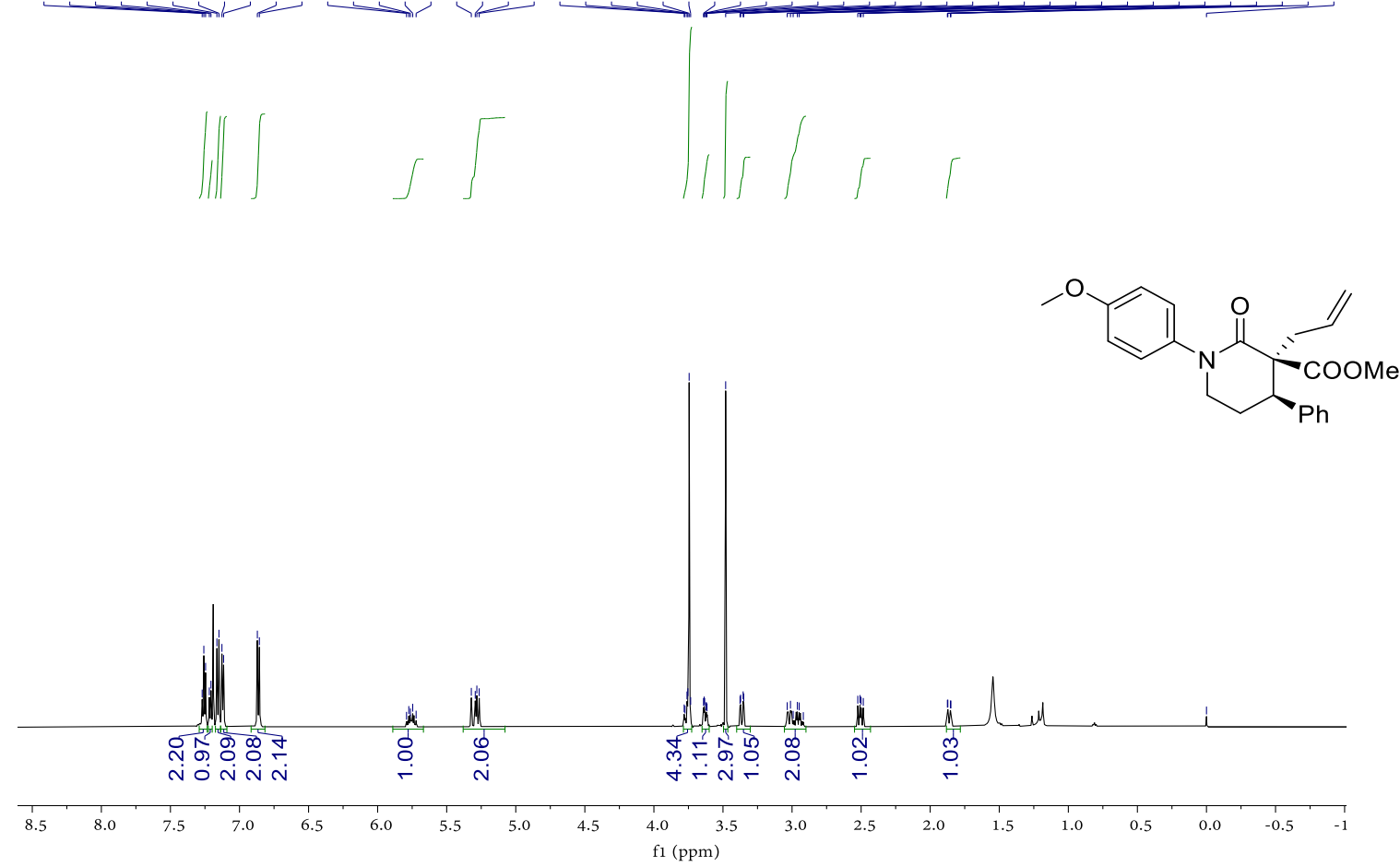

${ }^{13} \mathrm{C}\left\{{ }^{1} \mathrm{H}\right\}$ NMR (101 MHz, $\left.\mathrm{CDCl}_{3}\right)$ of compound 3b

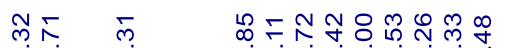

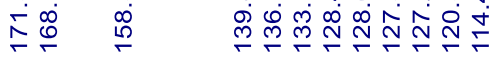
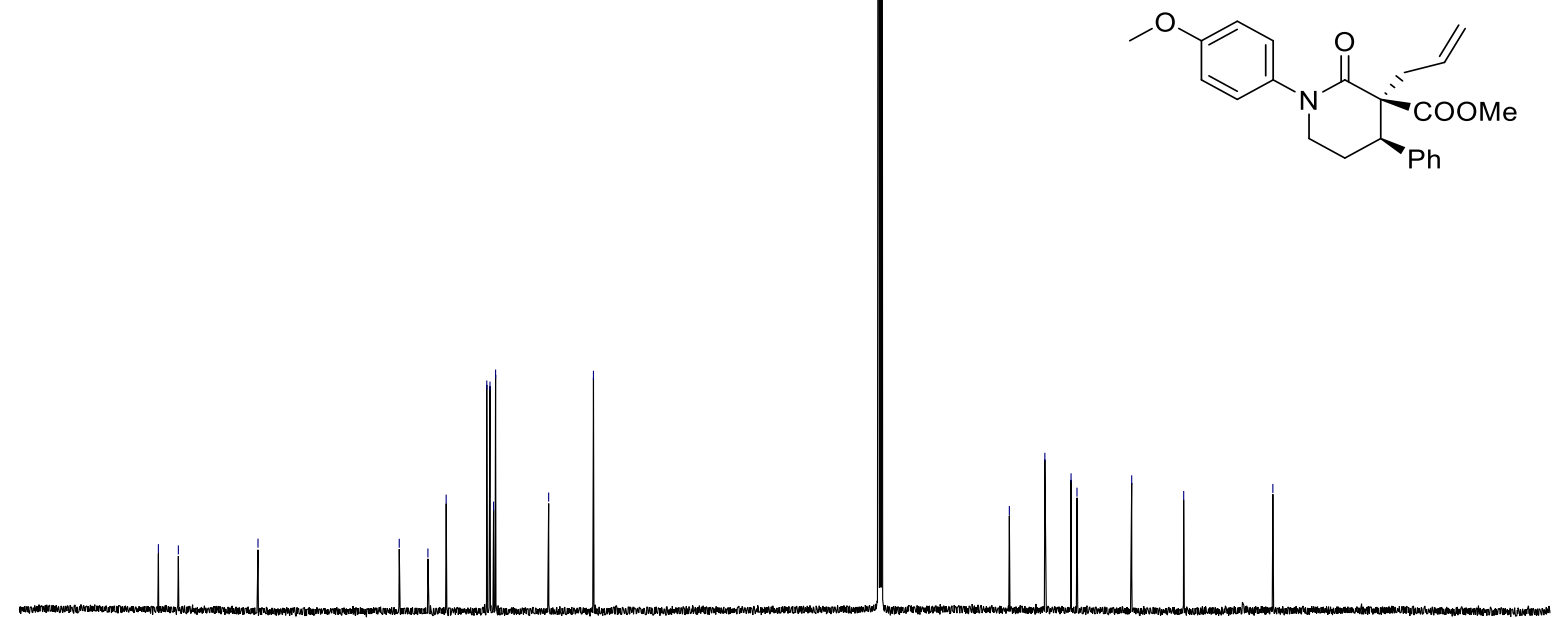

$\begin{array}{llllllllll}180 & 170 & 160 & 150 & 140 & 130 & 120 & 110 & 100 & 90 \\ \mathrm{f} 1(\mathrm{ppm})\end{array}$ 


\section{${ }^{1} \mathrm{H}$ NMR (600 MHz, $\mathrm{CDCl}_{3}$ ) of compound 3c}

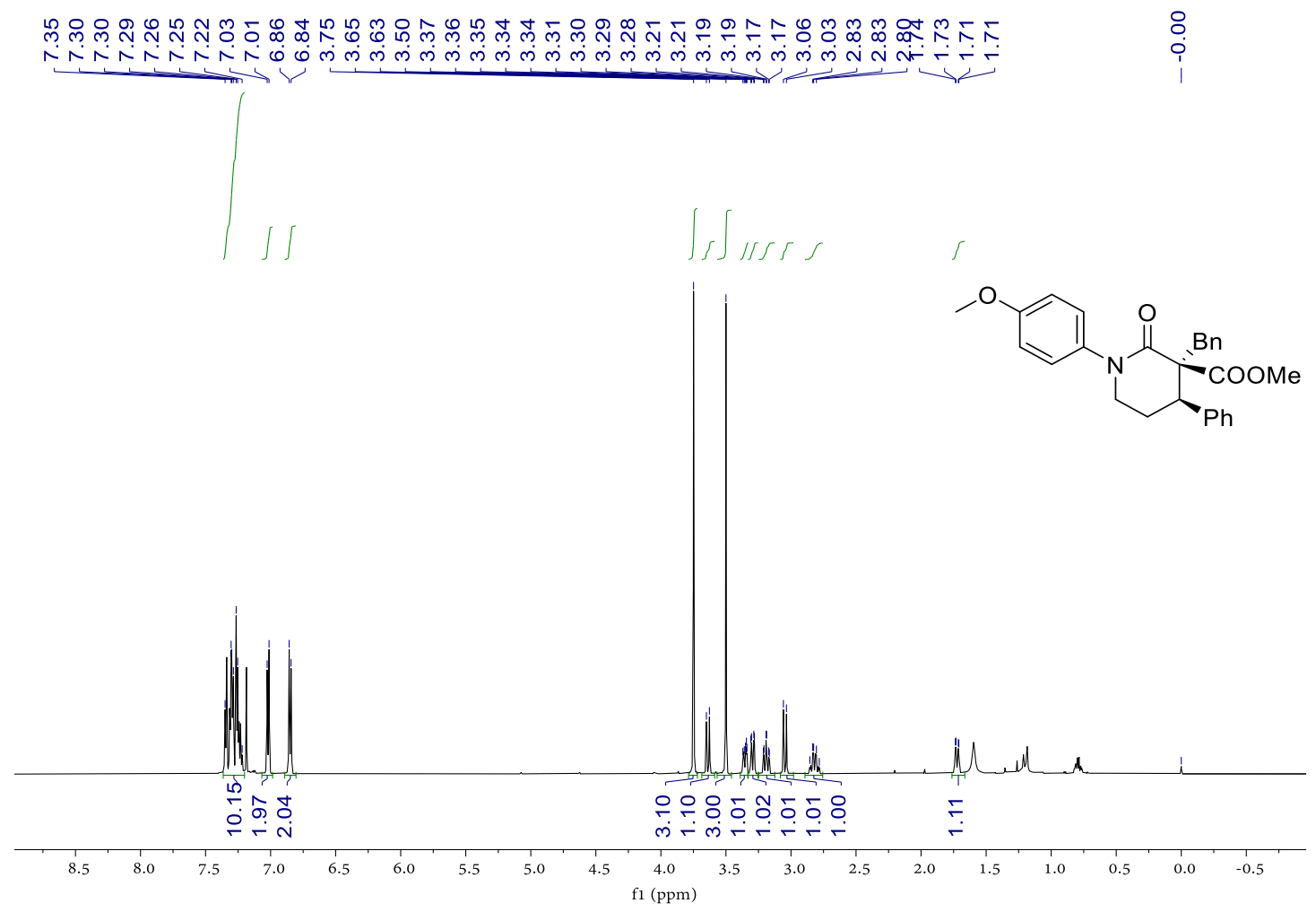

${ }^{13} \mathrm{C}\left\{{ }^{1} \mathrm{H}\right\}$ NMR (101 MHz, $\left.\mathrm{CDCl}_{3}\right)$ of compound 3c

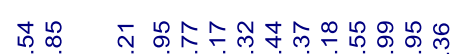

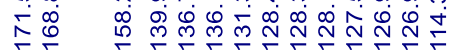

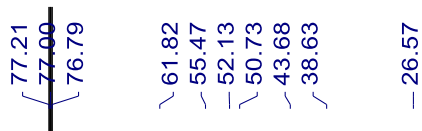
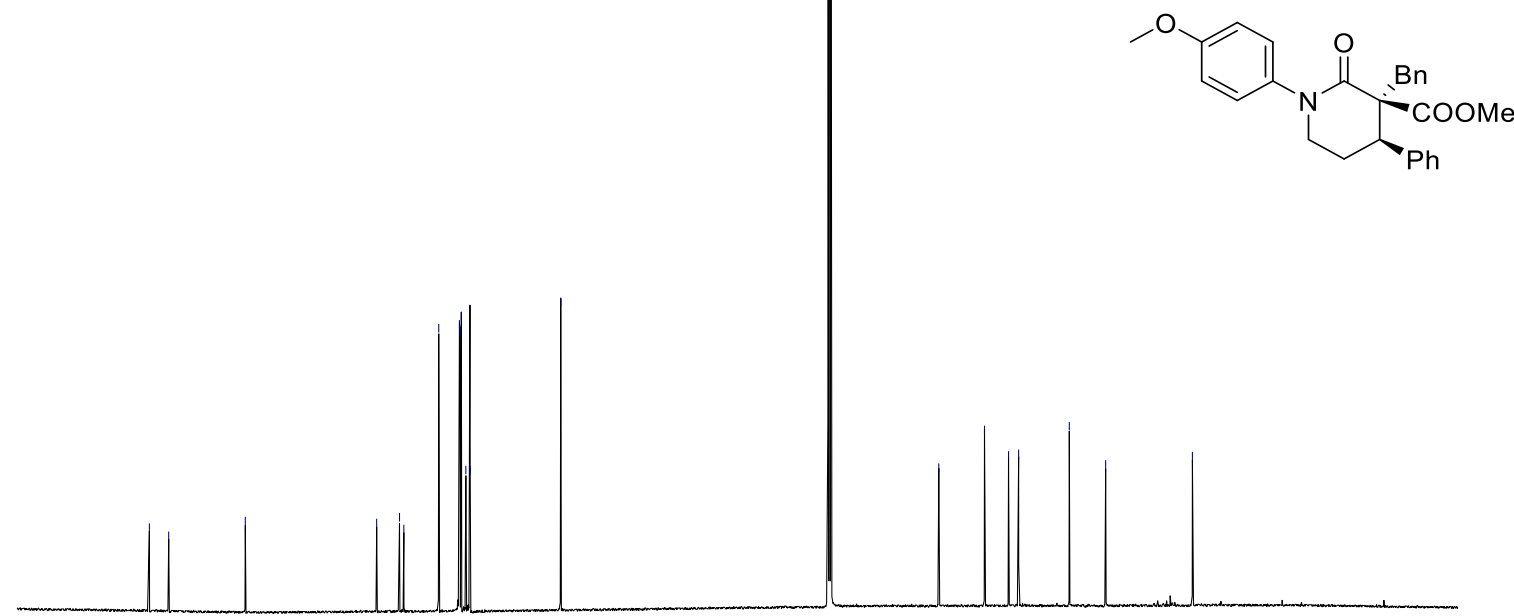

$\begin{array}{llllllllll}180 & 170 & 160 & 150 & 140 & 130 & 120 & 110 & 100 & 90 \\ & & & & & & & & & \text { f1 (ppm) }\end{array}$ 


\section{Crystal structure of 2c (CCDC 2112114)}

To determine the absolute configuration of 2c: firstly, 2 c (91\% ee, 16/1 dr) was upgraded to $99 \%$ ee and $>20 / 1 \mathrm{dr}$ by recrystallization with $n$-hexane/dichloromethane; $2 \mathbf{c}$ was then completely dissolved in minimum isopropyl alcohol in a $2 \mathrm{~mL}$ tube, followed by addition of 3 drops of $n$ hexane slowly. The solvent was slowly evaporated and the single crystal was obtained after two days. (Ellipsoid contour probability levels: 50\%)

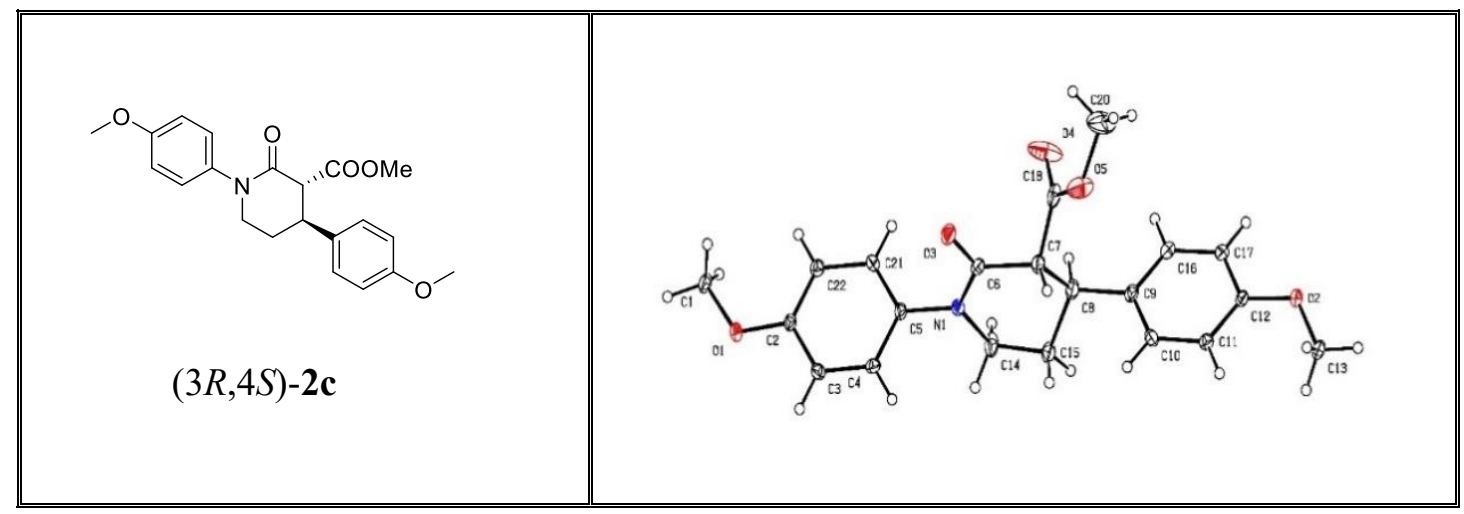

Identification code

Empirical formula

Formula weight

Temperature/K

Crystal system

Space group

$\mathrm{a} / \AA$

$\mathrm{b} / \AA$

$\mathrm{c} / \AA \AA$

$\alpha /{ }^{\circ}$

$\beta /{ }^{\circ}$

$\gamma /{ }^{\circ}$

Volume $/ \AA^{3}$

Z

$\rho c a l c g / \mathrm{cm}^{3}$

$\mu / \mathrm{mm}^{-1}$

\section{CCDC 2112114}

$\mathrm{C}_{21} \mathrm{H}_{23} \mathrm{NO}_{5}$

369.40

100.0

triclinic

P1

7.2654(4)

11.7175(7)

12.5932(7)

109.218(2)

105.540(2)

101.236(2)

926.93(9)

2

1.324

0.776 


\begin{tabular}{|c|c|}
\hline $\mathrm{F}(000)$ & 392.0 \\
\hline Crystal size $/ \mathrm{mm}^{3}$ & $0.39 \times 0.31 \times 0.25$ \\
\hline Radiation & $\mathrm{CuK} \alpha(\lambda=1.54178)$ \\
\hline $2 \Theta$ range for data collection $/{ }^{\circ}$ & 7.964 to 136.734 \\
\hline Index ranges & $-8 \leq \mathrm{h} \leq 8,-14 \leq \mathrm{k} \leq 14,-15 \leq 1 \leq 15$ \\
\hline Reflections collected & 24925 \\
\hline Independent reflections & $6599[$ Rint $=0.0322$, Rsigma $=0.0303]$ \\
\hline Data/restraints/parameters & $6599 / 3 / 493$ \\
\hline Goodness-of-fit on $\mathrm{F}^{2}$ & 1.032 \\
\hline Final $R$ indexes $[\mathrm{I}>=2 \sigma(\mathrm{I})]$ & $\mathrm{R} 1=0.0473, \mathrm{wR} 2=0.1254$ \\
\hline Final $\mathrm{R}$ indexes [all data] & $\mathrm{R} 1=0.0475, \mathrm{wR} 2=0.1256$ \\
\hline Largest diff. peak/hole / e $\AA^{-3}$ & $0.78 /-0.37$ \\
\hline Flack parameter & $-0.01(6)$ \\
\hline Correction method $=\#$ Reported & Limits: Tmin $=0.661 \quad$ Tmax $=0.753$ \\
\hline \multicolumn{2}{|l|}{ AbsCorr $=$ MULTI-SCAN } \\
\hline Data completeness $=1.94 / 0.97$ & $\operatorname{Theta}(\max )=68.367$ \\
\hline $\mathrm{R}($ reflections $)=0.0473(6547)$ & $\mathrm{wR} 2($ reflections $)=0.1256(6599)$ \\
\hline $\mathrm{S}=1.032$ & Npar $=493$ \\
\hline
\end{tabular}




\section{HPLC spectra}

Methyl (3R,4S)-1-(4-methoxyphenyl)-2-oxo-4-phenylpiperidine-3-carboxylate

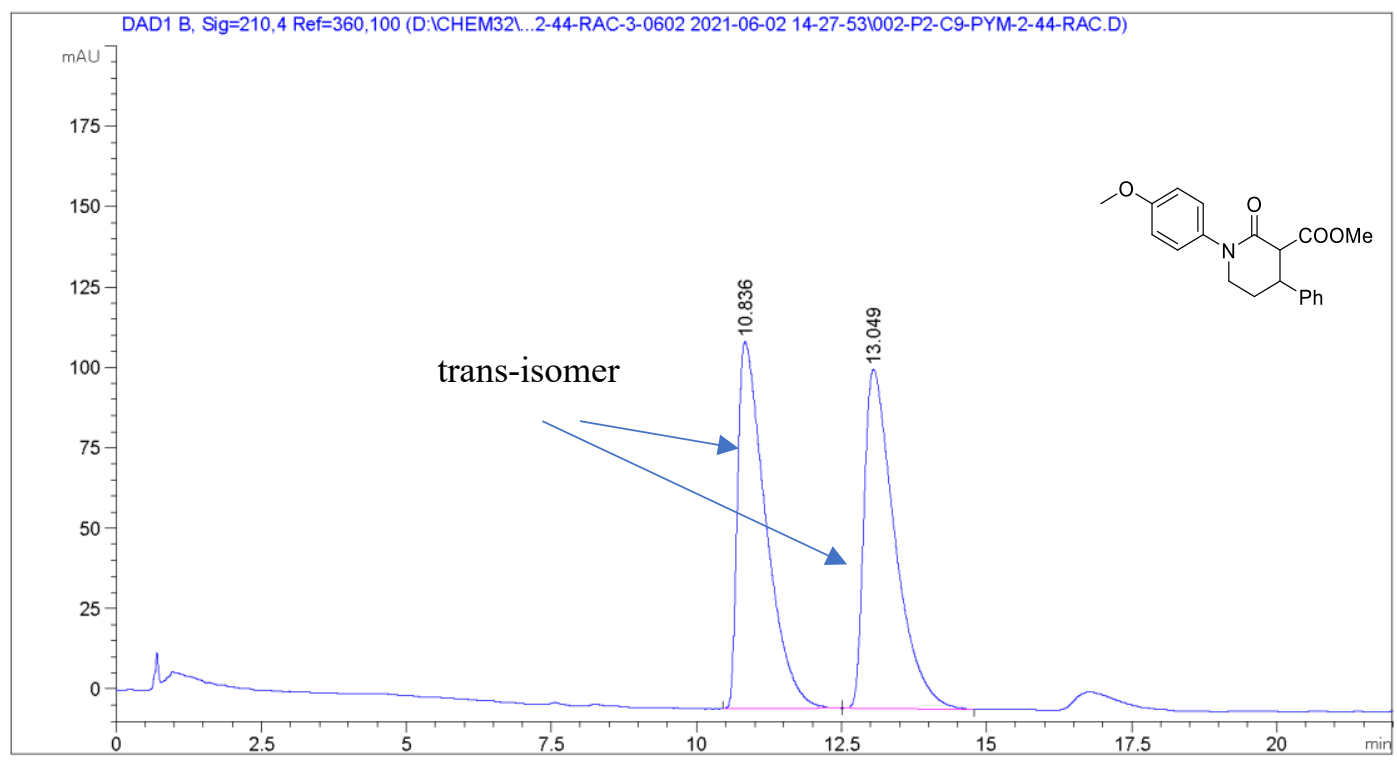

Area Percent Report

\begin{tabular}{|c|c|c|c|c|}
\hline Sorted By & : & Signal & & \\
\hline Multiplier & : & 1.0000 & & \\
\hline Dilution & : & 1.0000 & & \\
\hline \multicolumn{5}{|c|}{ Use Multiplier \& Dilution Factor with ISTDs } \\
\hline \multicolumn{5}{|c|}{ Signal 1: DAD1 B, Sig=210,4 $\operatorname{Re} f=360,100$} \\
\hline $\begin{array}{l}\text { Peak RetTime Type } \\
\# \quad[\text { min] }\end{array}$ & $\begin{array}{l}\text { Width } \\
\text { [min] }\end{array}$ & $\begin{array}{c}\text { Area } \\
{[\mathrm{mAU} * \mathrm{~s}]}\end{array}$ & $\begin{array}{l}\text { Height } \\
{[\mathrm{mAU}]}\end{array}$ & $\begin{array}{c}\text { Area } \\
\%\end{array}$ \\
\hline$-\ldots|---1----|$ & 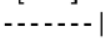 & $|-\ldots-1-2|$ & | & 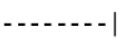 \\
\hline 110.836 BB & 0.4879 & 3849.85645 & 114.02904 & 49.7263 \\
\hline $213.049 \mathrm{BB}$ & 0.5551 & 3892.23120 & 105.33997 & 50.2737 \\
\hline Totals : & & 7742.08765 & 219.36901 & \\
\hline
\end{tabular}




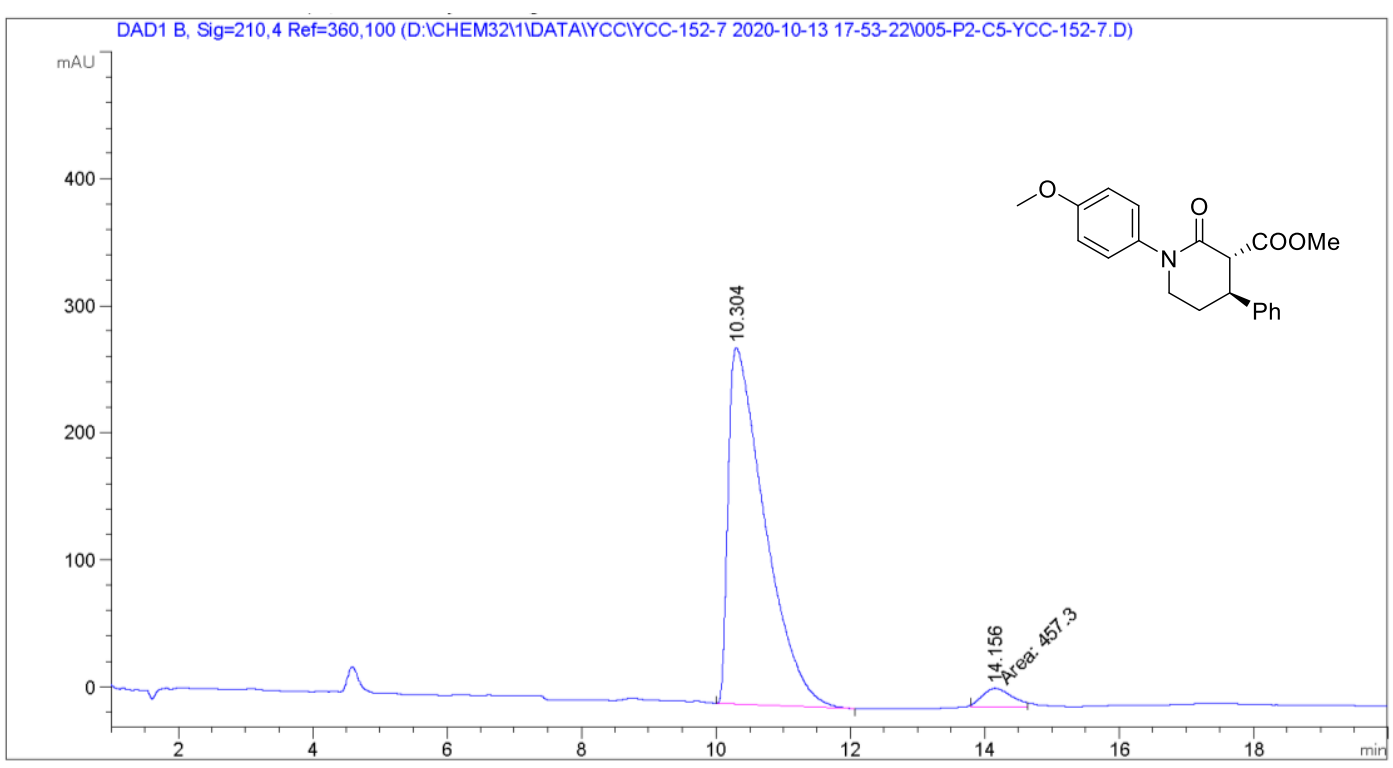

Area Percent Report

$\begin{array}{lll}\text { Sorted By } & : & \text { Signal } \\ \text { Multiplier } & : & 1.0000 \\ \text { Dilution } & : & 1.0000\end{array}$

Use Multiplier \& Dilution Factor with ISTDs

Signal 1: DAD1 B, Sig $=210,4$ Ref $=360,100$

\begin{tabular}{|c|c|c|c|c|c|c|}
\hline $\begin{array}{c}\text { Peak } \\
\#\end{array}$ & $\begin{array}{c}\text { RetTime } \\
\text { [min] }\end{array}$ & Type & $\begin{array}{l}\text { Width } \\
\text { [min] }\end{array}$ & $\begin{array}{c}\text { Area } \\
{\left[\mathrm{mAU}^{*} \mathrm{~s}\right]}\end{array}$ & $\begin{array}{l}\text { Height } \\
{[\mathrm{mAU}]}\end{array}$ & $\begin{array}{c}\text { Area } \\
\%\end{array}$ \\
\hline$\ldots$ & |- & & - & | & - & 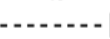 \\
\hline 1 & 10.304 & BB & 0.5656 & $1.05611 \mathrm{e} 4$ & 280.29236 & 95.8497 \\
\hline 2 & 14.156 & MM & 0.5170 & 457.30017 & 14.74087 & 4.1503 \\
\hline Tota & : & & & $1.10184 \mathrm{e} 4$ & 295.03323 & \\
\hline
\end{tabular}


Ethyl (3R,4S)-1-(4-methoxyphenyl)-2-oxo-4-phenylpiperidine-3-carboxylate 2b

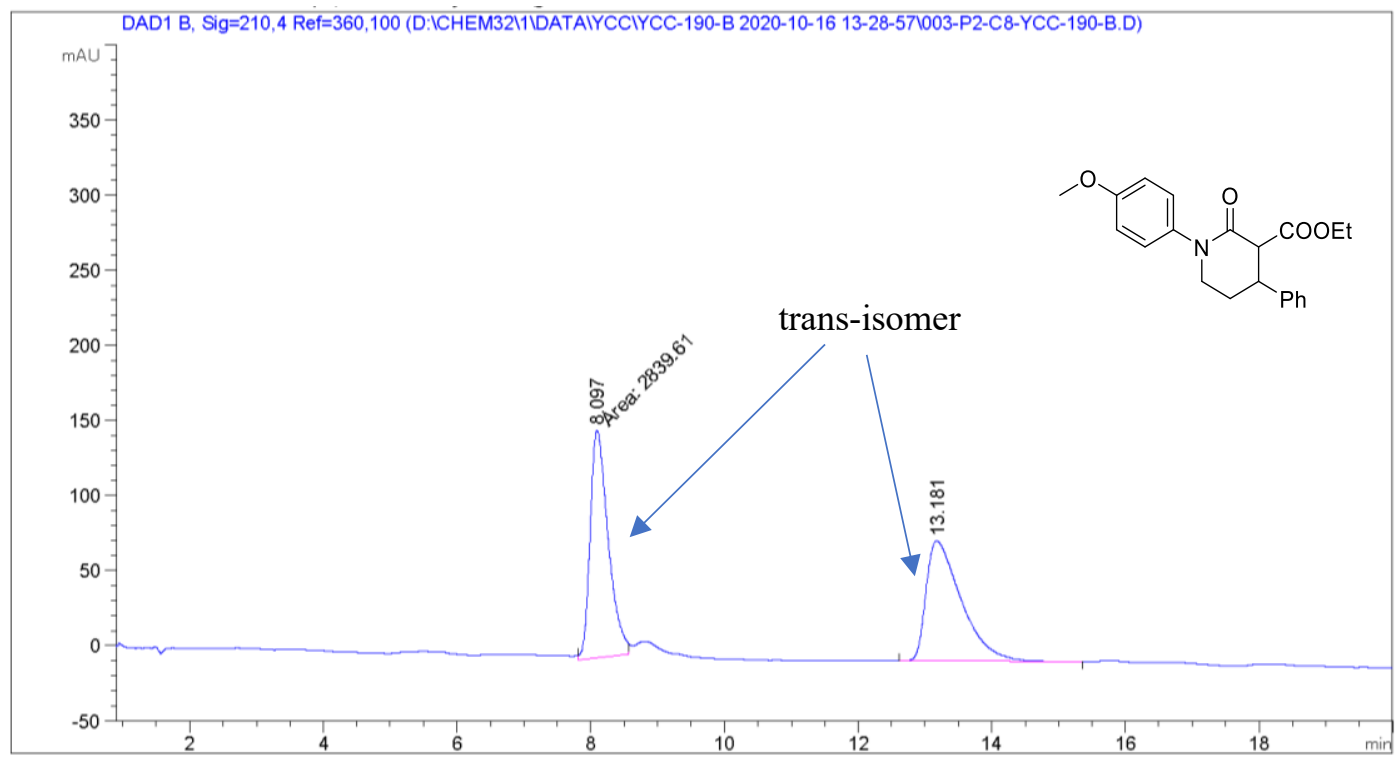

Area Percent Report

\begin{tabular}{|c|c|c|c|c|}
\hline Sorted By & $:$ & Signal & & \\
\hline Multiplier & : & 1.0000 & & \\
\hline Dilution & : & 1.0000 & & \\
\hline \multicolumn{5}{|c|}{ Use Multiplier \& Dilution Factor with ISTDs } \\
\hline \multicolumn{5}{|c|}{ Signal 1: DAD1 B, Sig=210,4 Ref=360,100 } \\
\hline $\begin{array}{l}\text { Peak RetTime Type } \\
\# \quad[\text { min] }\end{array}$ & $\begin{array}{l}\text { Width } \\
\text { [min] }\end{array}$ & $\begin{array}{c}\text { Area } \\
{[\mathrm{mAU} * \mathrm{~s}]}\end{array}$ & $\begin{array}{l}\text { Height } \\
{[\mathrm{mAU}]}\end{array}$ & $\begin{array}{c}\text { Area } \\
\%\end{array}$ \\
\hline $\mid$ & 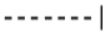 & $|-\infty-\infty|$ & | & | \\
\hline $1 \quad 8.097$ MM & 0.3126 & 2839.61060 & 151.37575 & 49.3120 \\
\hline 213.181 BB & 0.5541 & 2918.85132 & 79.91167 & 50.6880 \\
\hline Totals : & & 5758.46191 & 231.28742 & \\
\hline
\end{tabular}

1290-DAD 7/9/2021 2:41:45 PM SYSTEM 


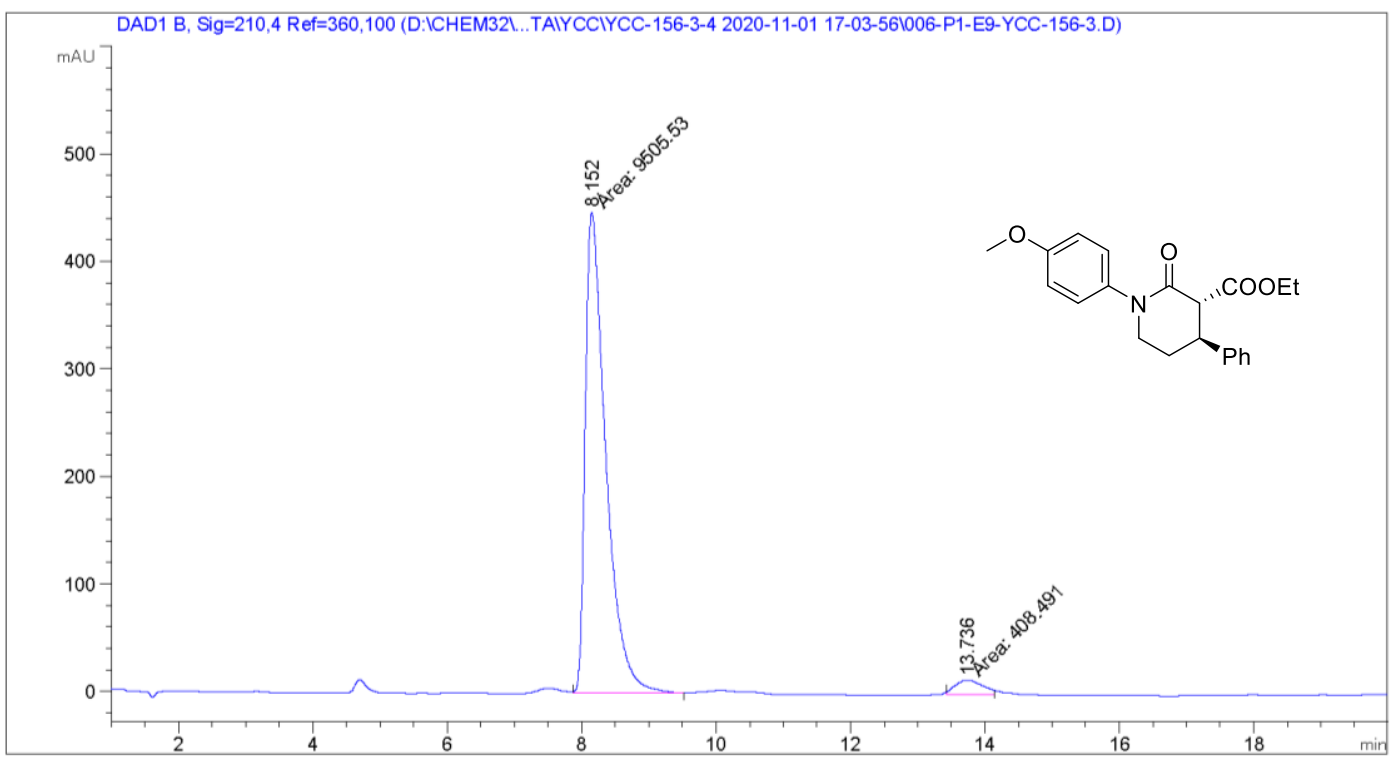

Area Percent Report

Sorted By : : Signal

Multiplier : $\quad 1.0000$

Dilution : 1.0000

Use Multiplier \& Dilution Factor with ISTDs

Signal 1: DAD1 B, Sig $=210,4$ Ref $=360,100$

\begin{tabular}{cccccc}
$\begin{array}{c}\text { Peak RetTime Type } \\
\text { Width } \\
\text { [min] }\end{array}$ & $\begin{array}{c}\text { Area } \\
{[\text { min] }}\end{array}$ & $\begin{array}{c}\text { Height } \\
{[\text { mAU*s] }}\end{array}$ & $\begin{array}{c}\text { Area } \\
{[\mathrm{mAU}]}\end{array}$ & $\%$ \\
\hline 1 & $8.152 \mathrm{FM}$ & 0.3542 & 9505.52930 & 447.28174 & 95.8797 \\
2 & $13.736 \mathrm{MM}$ & 0.4872 & 408.49121 & 13.97373 & 4.1203 \\
& & & & \\
Totals : & & 9914.02051 & 461.25547
\end{tabular}


Methyl (3R,4S)-1,4-bis(4-methoxyphenyl)-2-oxopiperidine-3-carboxylate 2c

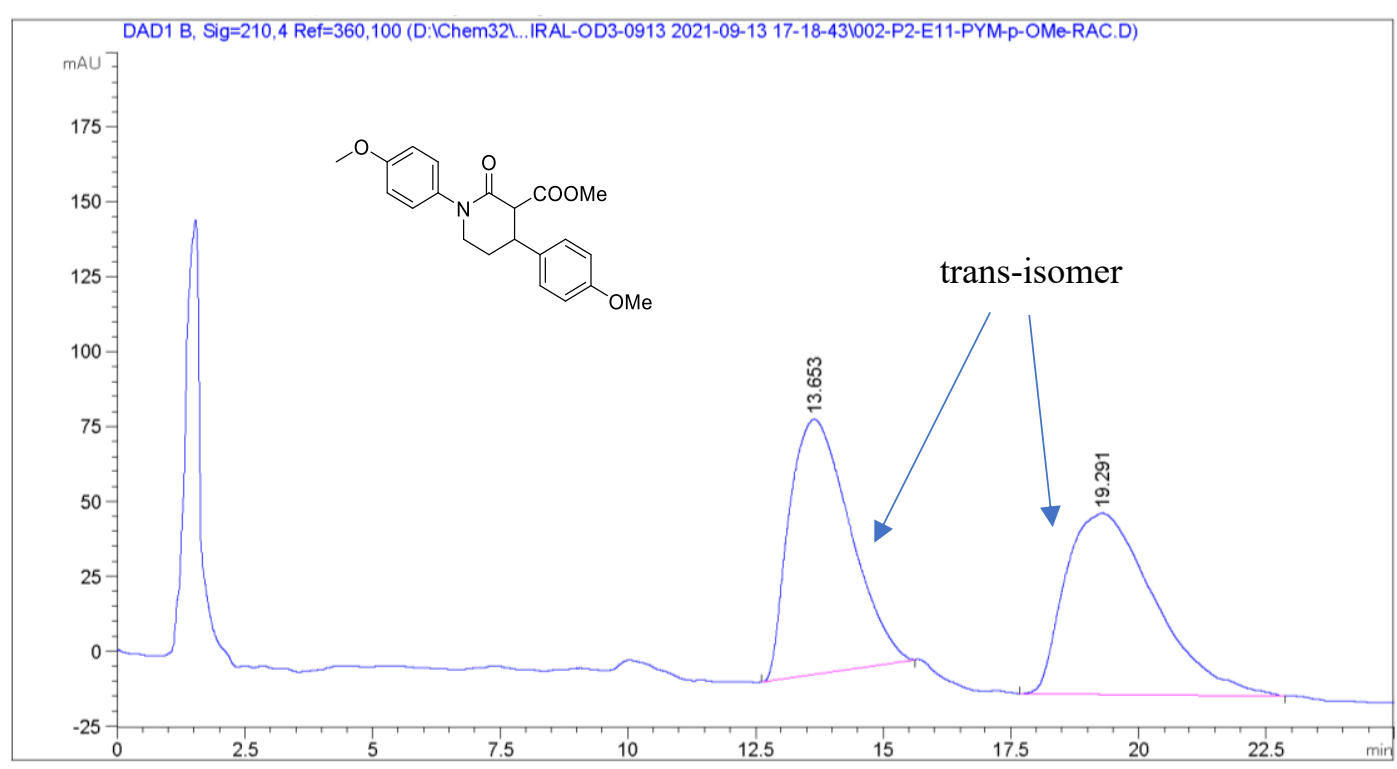

Area Percent Report

$\begin{array}{lll}\text { Sorted By } & : & \text { Signal } \\ \text { Multiplier } & : & 1.0000 \\ \text { Dilution } & : & 1.0000\end{array}$

Use Multiplier \& Dilution Factor with ISTDs

Signal 1: DAD1 B, Sig=210,4 Ref $=360,100$

\begin{tabular}{|c|c|c|c|c|c|}
\hline $\begin{array}{c}\text { Peak } \\
\#\end{array}$ & $\begin{array}{l}\text { RetTime Type } \\
\text { [min] }\end{array}$ & $\begin{array}{l}\text { Width } \\
\text { [min] }\end{array}$ & $\begin{array}{c}\text { Area } \\
{[\mathrm{mAU} * \mathrm{~s}]}\end{array}$ & $\begin{array}{l}\text { Height } \\
\text { [mAU] }\end{array}$ & $\begin{array}{c}\text { Area } \\
\%\end{array}$ \\
\hline & & & - & & \\
\hline 1 & $13.653 \mathrm{BB}$ & 1.2307 & 7060.62793 & 85.21503 & 48.9850 \\
\hline 2 & 19.291 BB & 1.5135 & 7353.22119 & 60.43614 & 51.0150 \\
\hline
\end{tabular}




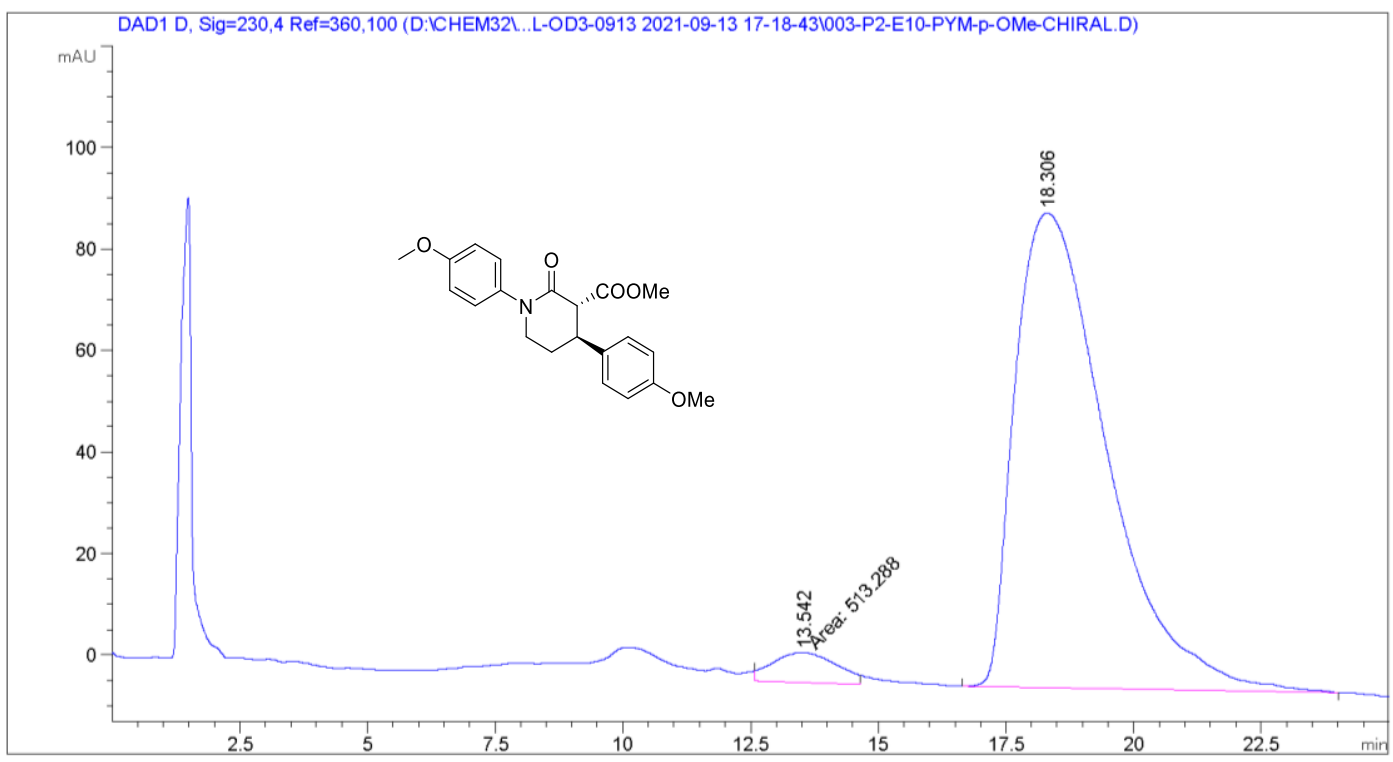

Area Percent Report

Sorted By : : Signal

Multiplier : $\quad 1.0000$

Dilution : 1.0000

Use Multiplier \& Dilution Factor with ISTDs

Signal 1: DAD1 D, Sig $=230,4$ Ref $=360,100$

\begin{tabular}{|c|c|c|c|c|c|}
\hline $\begin{array}{c}\text { Peak } \\
\#\end{array}$ & $\begin{array}{l}\text { RetTime Type } \\
{[\mathrm{min}]}\end{array}$ & $\begin{array}{l}\text { Width } \\
\text { [min] }\end{array}$ & $\begin{array}{c}\text { Area } \\
{\left[\mathrm{mAU}^{*} \mathrm{~s}\right]}\end{array}$ & $\begin{array}{l}\text { Height } \\
\text { [mAU] }\end{array}$ & $\begin{array}{c}\text { Area } \\
\%\end{array}$ \\
\hline$\cdots$ & - & 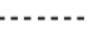 & |- & | & | \\
\hline 1 & $13.542 \mathrm{MM}$ & 1.4623 & 513.28760 & 5.85039 & 4.2183 \\
\hline 2 & 18. 306 BB & 1.9288 & $1.16549 \mathrm{e} 4$ & 93.46690 & 95.7817 \\
\hline Tota & : & & $1.21682 \mathrm{e} 4$ & 99.31729 & \\
\hline
\end{tabular}




\section{$2 \mathrm{c}$ after crystallization}

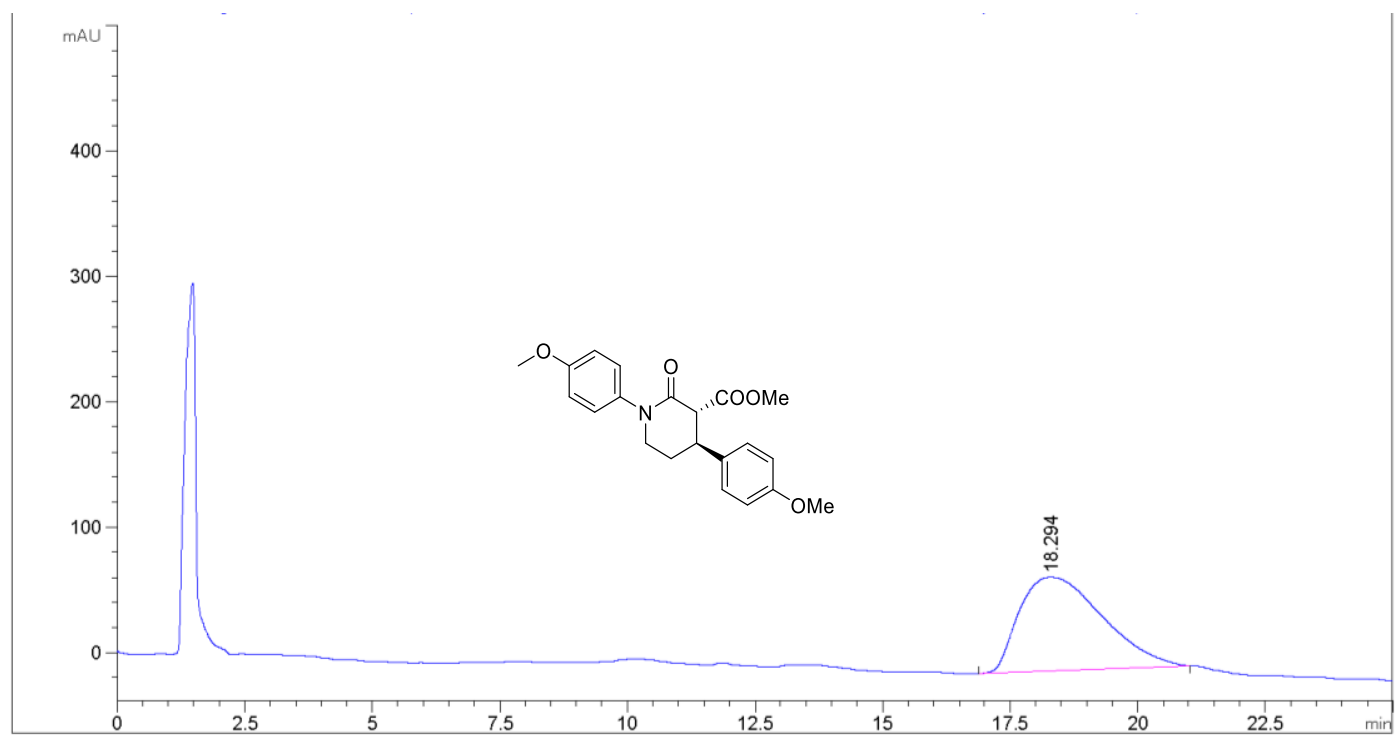

Area Percent Report

$\begin{array}{lll}\text { Sorted By } & : & \text { Signal } \\ \text { Multiplier } & : & 1.0000 \\ \text { Dilution } & : & 1.0000\end{array}$

Use Multiplier \& Dilution Factor with ISTDs

Signal 1: DAD1 B, Sig=210,4 $\operatorname{Re} f=360,100$

\begin{tabular}{|c|c|c|c|c|}
\hline $\begin{array}{l}\text { Peak RetTime Type } \\
\# \quad \text { [min] }\end{array}$ & $\begin{array}{l}\text { Width } \\
{[\mathrm{min}]}\end{array}$ & $\begin{array}{c}\text { Area } \\
{\left[\mathrm{mAU}^{*} \mathrm{~s}\right]}\end{array}$ & $\begin{array}{l}\text { Height } \\
\text { [mAU] }\end{array}$ & $\begin{array}{c}\text { Area } \\
\%\end{array}$ \\
\hline 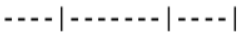 & $-\ldots$ & | & |- & - \\
\hline $118.294 \mathrm{BB}$ & 1.5756 & 8479.85938 & 74.85693 & 100.0000 \\
\hline Totals : & & 8479.85938 & 74.85693 & \\
\hline
\end{tabular}


Methyl (3R,4S)-4-(4-fluorophenyl)-1-(4-methoxyphenyl)-2-oxopiperidine-3-carboxylate

2d

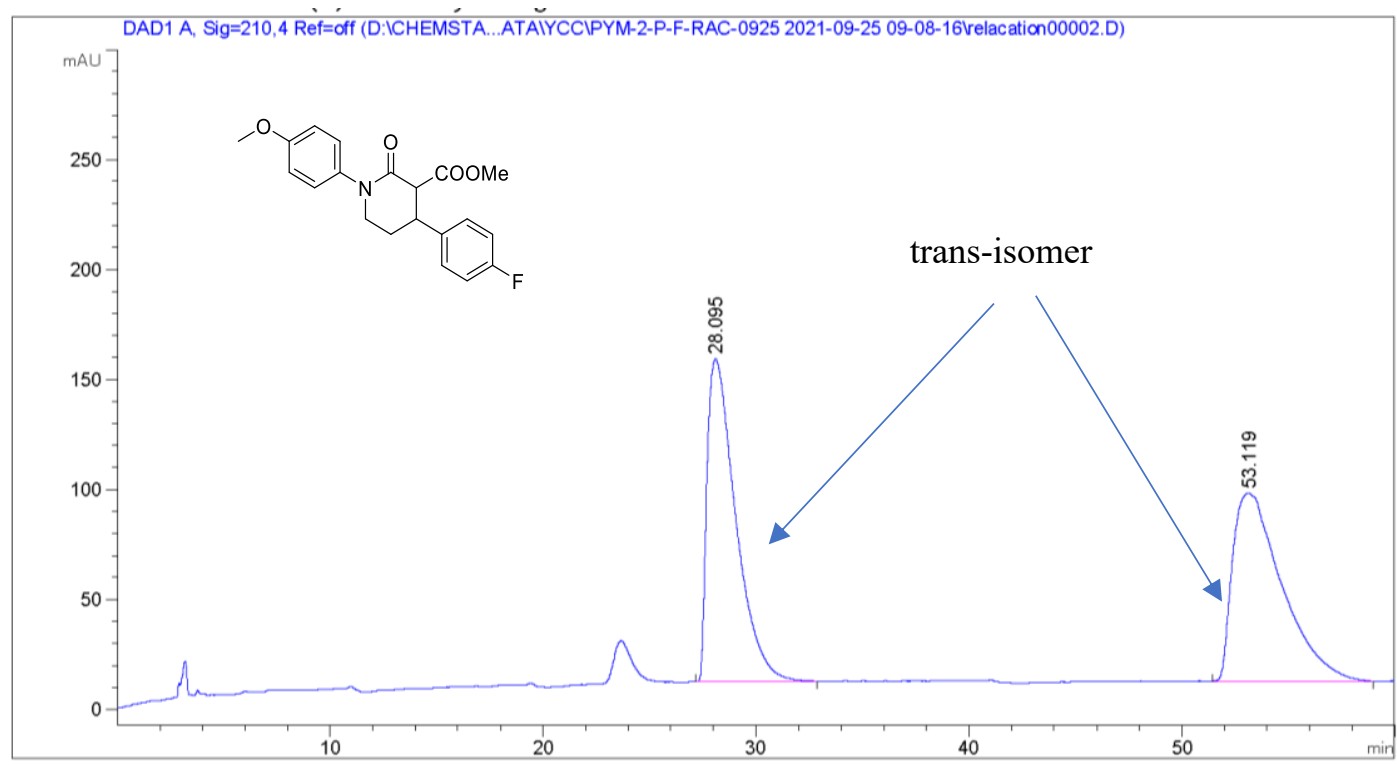

Area Percent Report

$\begin{array}{lcc}\text { Sorted By } & : & \text { Signal } \\ \text { Multiplier } & : & 1.0000 \\ \text { Dilution } & : & 1.0000 \\ \text { Use Multiplier } & \text { \& Dilution } & \text { Factor with }\end{array}$

Signal 1: DAD1 A, Sig=210,4 Ref=off

\begin{tabular}{cccccc}
$\begin{array}{c}\text { Peak RetTime Type Width } \\
\text { \# [min] }\end{array}$ [min] & $\begin{array}{c}\text { Area } \\
{[\text { mAU*s] }}\end{array}$ & $\begin{array}{c}\text { Height } \\
{[\mathrm{mAU}]}\end{array}$ & $\begin{array}{c}\text { Area } \\
\%\end{array}$ \\
\hdashline 1 & 28.095 BB & 1.3761 & $1.37128 \mathrm{e} 4$ & 146.75618 & 49.9176 \\
2 & 53.119 BB & 1.9115 & $1.37580 \mathrm{e} 4$ & 85.40144 & 50.0824
\end{tabular}




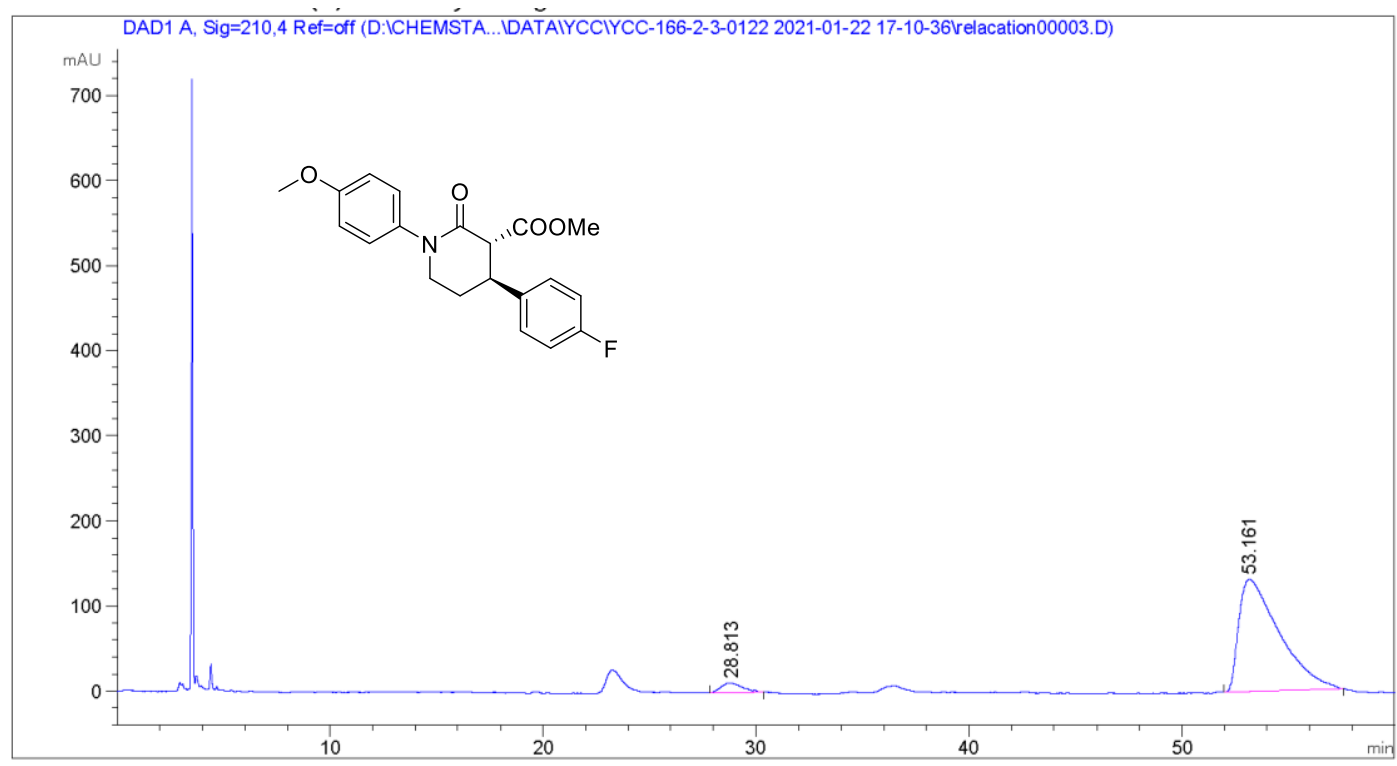

Area Percent Report

\begin{tabular}{|c|c|c|c|c|}
\hline Sorted By & $:$ & Signal & & \\
\hline Multiplier & $:$ & 1.0000 & & \\
\hline Dilution & : & 1.0000 & & \\
\hline \multicolumn{5}{|c|}{ Use Multiplier \& Dilution Factor with ISTDs } \\
\hline \multicolumn{5}{|c|}{ Signal 1: DAD1 A, Sig $=210,4$ Ref $=0 f f$} \\
\hline $\begin{array}{l}\text { Peak RetTime Type } \\
\# \quad[\text { min] }\end{array}$ & $\begin{array}{l}\text { Width } \\
\text { [min] }\end{array}$ & $\begin{array}{c}\text { Area } \\
{[\mathrm{mAU} * \mathrm{~s}]}\end{array}$ & $\begin{array}{l}\text { Height } \\
{[\mathrm{mAU}]}\end{array}$ & $\begin{array}{c}\text { Area } \\
\%\end{array}$ \\
\hline$---|----|--\mid$ & $-\cdots$ & $|-\ldots-n|$ & | & \\
\hline 128.813 BV R & 0.8310 & 804.86084 & 11.18083 & 4.4120 \\
\hline $253.161 \mathrm{BB}$ & 1.5590 & $1.74379 \mathrm{e} 4$ & 132.01932 & 95.5880 \\
\hline
\end{tabular}


Methyl (3R,4S)-1-(4-methoxyphenyl)-2-oxo-4-(m-tolyl)piperidine-3-carboxylate 2e

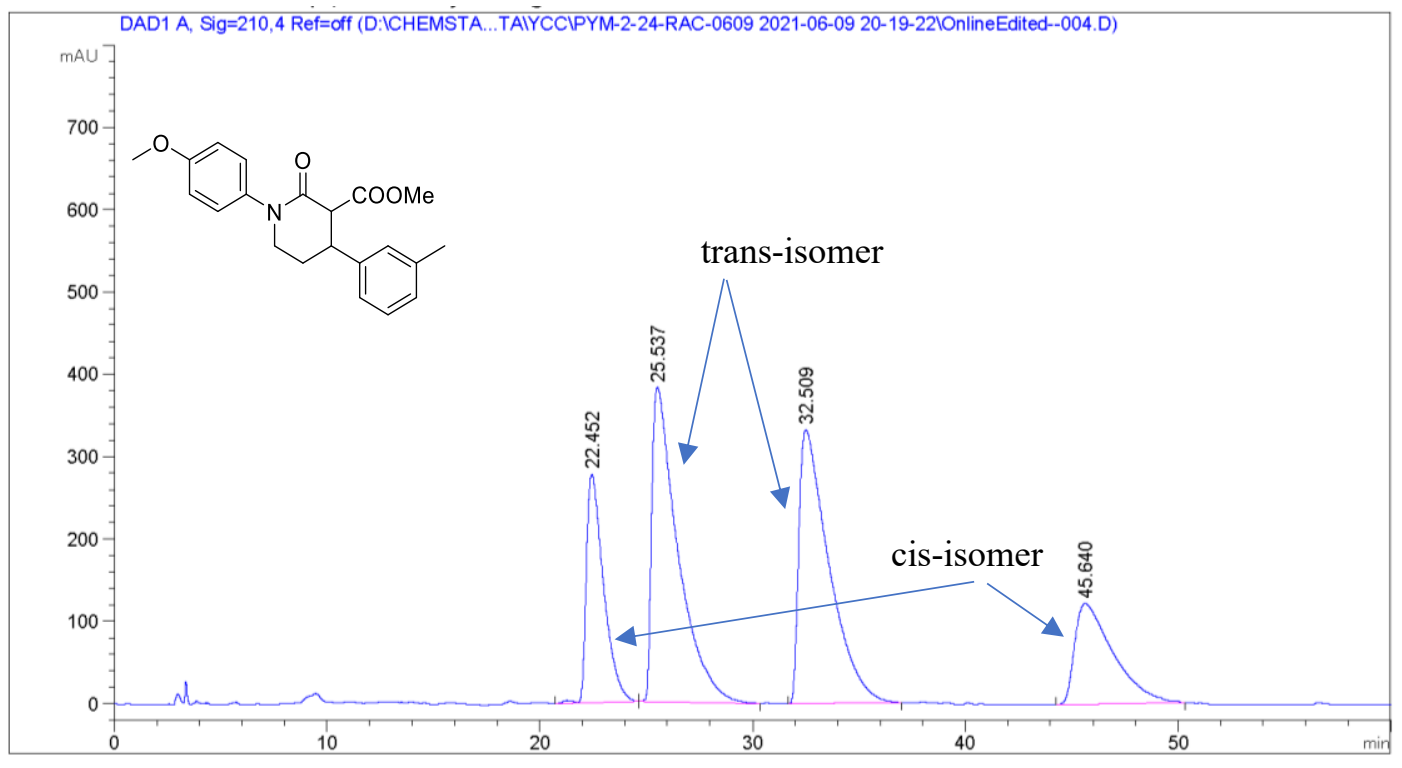

Area Percent Report

$\begin{array}{lll}================================== \\ \text { Sorted By } & : & \text { Signal } \\ \text { Multiplier } & : & 1.0000 \\ \text { Dilution } & : & 1.0000\end{array}$

Use Multiplier \& Dilution Factor with ISTDs

Signal 1: DAD1 A, Sig=210,4 Ref=off

\begin{tabular}{|c|c|c|c|c|c|c|}
\hline $\begin{array}{c}\text { Peak } \\
\#\end{array}$ & $\begin{array}{c}\text { RetTime } \\
\text { [min] }\end{array}$ & Type & $\begin{array}{l}\text { Width } \\
\text { [min] }\end{array}$ & $\begin{array}{c}\text { Area } \\
{\left[\mathrm{mAU}^{*} \mathrm{~s}\right]}\end{array}$ & $\begin{array}{l}\text { Height } \\
{[\mathrm{mAU}]}\end{array}$ & $\begin{array}{c}\text { Area } \\
\%\end{array}$ \\
\hline--1 & - - - & $|-\ldots|$ & 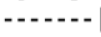 & | & - & | \\
\hline 1 & 22.452 & VB $R$ & 0.8455 & $1.56008 \mathrm{e} 4$ & 277.23108 & 16.2649 \\
\hline 2 & 25.537 & BB & 1.1911 & $3.23873 \mathrm{e} 4$ & 381.23392 & 33.7659 \\
\hline 3 & 32.509 & BB & 1.3549 & $3.25998 \mathrm{e} 4$ & 332.19995 & 33.9874 \\
\hline $\begin{array}{c}\text { Peak } \\
\#\end{array}$ & $\begin{array}{c}\text { RetTime } \\
\text { [min] }\end{array}$ & Type & $\begin{array}{l}\text { Width } \\
\text { [min] }\end{array}$ & $\begin{array}{c}\text { Area } \\
{[\mathrm{mAU} * \mathrm{~s}]}\end{array}$ & $\begin{array}{l}\text { Height } \\
{[\mathrm{mAU}]}\end{array}$ & $\begin{array}{c}\text { Area } \\
\%\end{array}$ \\
\hline---1 & |- - - - & | - - - & $-----\mid$ & | - - - - & -------- & $------\mid$ \\
\hline 4 & 45.640 & BB & 1.7310 & $1.53292 \mathrm{e} 4$ & 122.24168 & 15.9817 \\
\hline Total & Is : & & & $9.59171 \mathrm{e} 4$ & 1112.90662 & \\
\hline
\end{tabular}

*** End of Report *** 


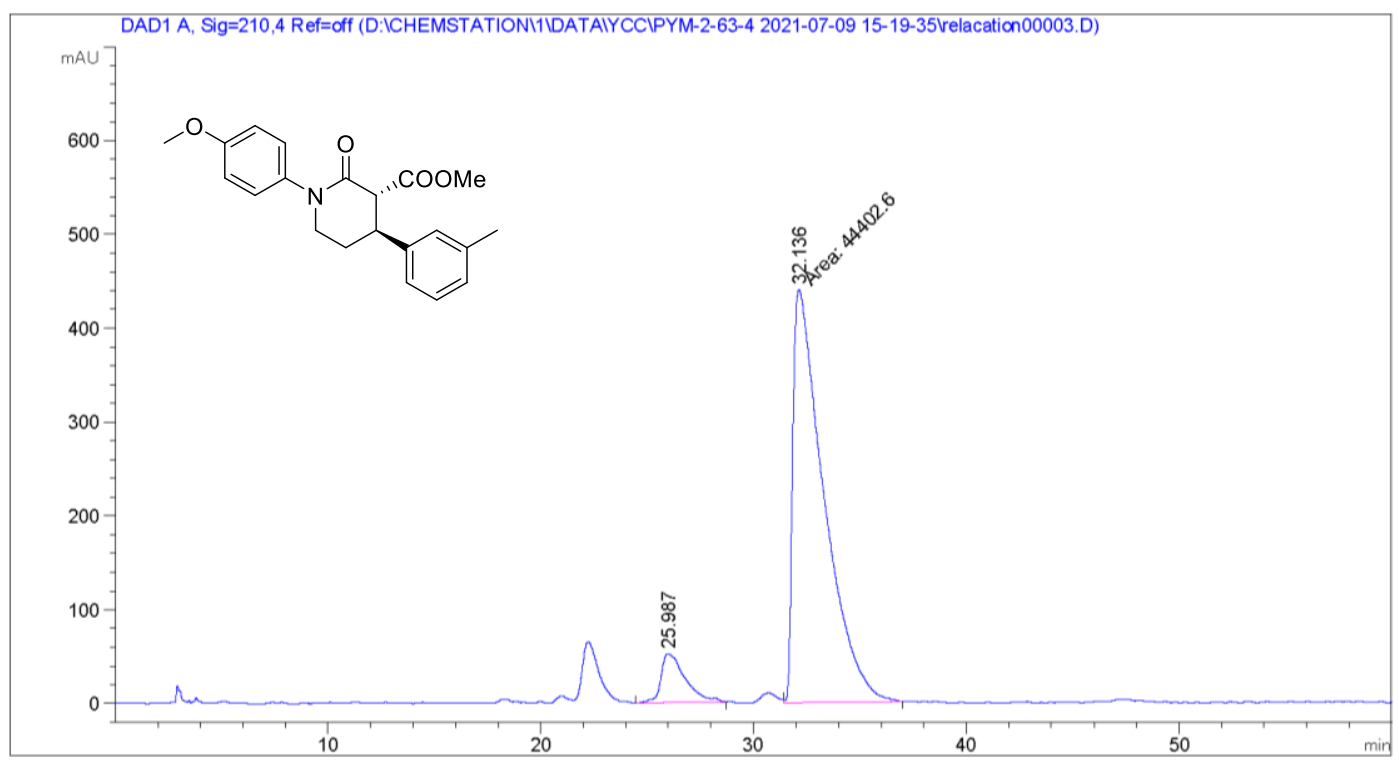

Area Percent Report

\begin{tabular}{|c|c|c|c|c|}
\hline Sorted By & : & Signal & & \\
\hline Multiplier & : & 1.0000 & & \\
\hline Dilution & : & 1.0000 & & \\
\hline \multicolumn{5}{|c|}{ Use Multiplier \& Dilution Factor with ISTDs } \\
\hline \multicolumn{5}{|c|}{ Signal 1: DAD1 A, Sig $=210,4$ Ref $=0 f f$} \\
\hline $\begin{array}{l}\text { Peak RetTime Type } \\
\# \quad[\text { min] }\end{array}$ & $\begin{array}{l}\text { Width } \\
\text { [min] }\end{array}$ & $\begin{array}{c}\text { Area } \\
{\left[\mathrm{mAU}^{*} \mathrm{~s}\right]}\end{array}$ & $\begin{array}{l}\text { Height } \\
\text { [mAU] }\end{array}$ & $\begin{array}{c}\text { Area } \\
\%\end{array}$ \\
\hline$---|-\cdots--|-n \mid$ & $-\cdots-1$ & $|--\cdots+-1|$ & $-\cdots-1$ & $-\cdots-1$ \\
\hline 125.987 BV R & 1.0394 & 4112.89893 & 52.13862 & 8.4775 \\
\hline $232.136 \mathrm{FM}$ & 1.6823 & $4.44026 \mathrm{e} 4$ & 439.90399 & 91.5225 \\
\hline
\end{tabular}




\section{Methyl (3R,4S)-1-benzyl-2-oxo-4-phenylpiperidine-3-carboxylate $2 \mathrm{f}$}

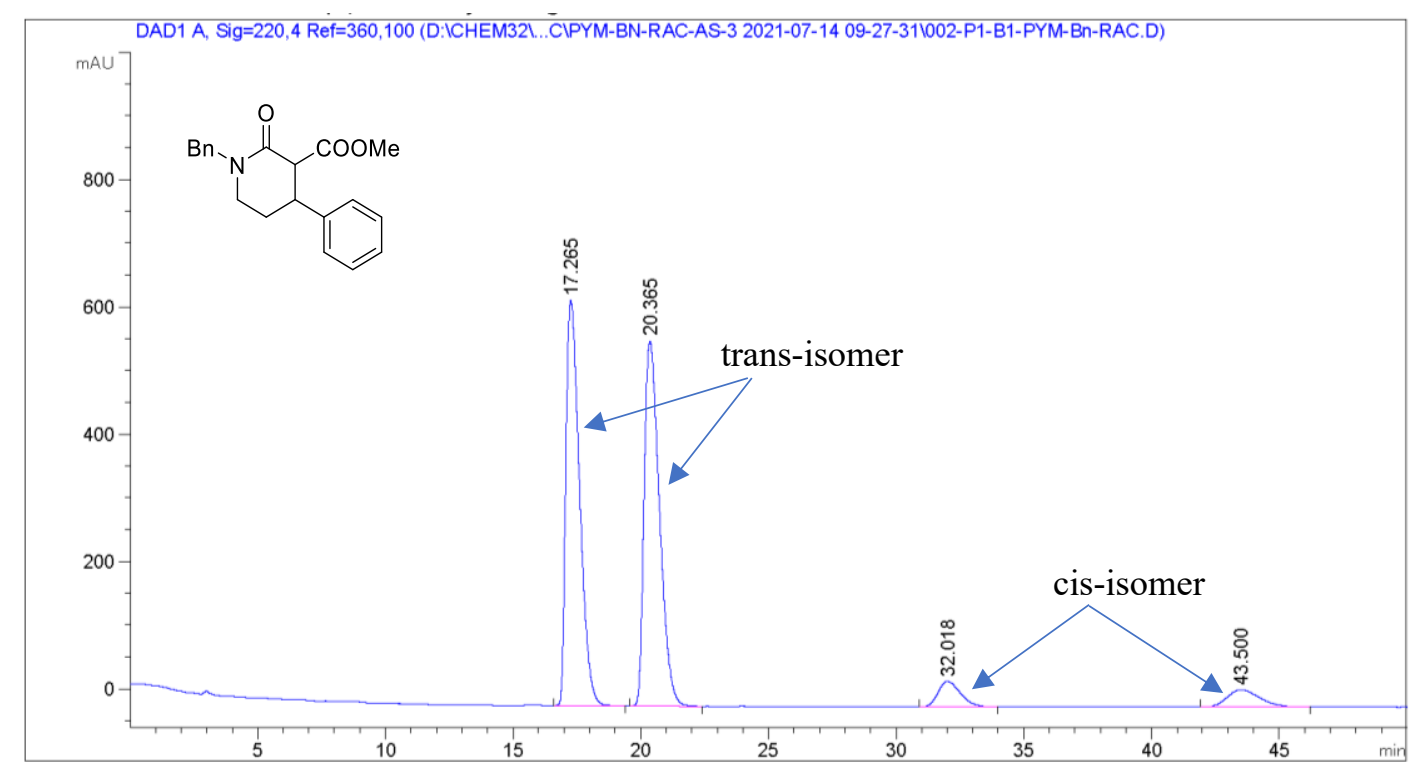

Area Percent Report

$\begin{array}{lll}\text { Sorted By } & : & \text { Signal } \\ \text { Multiplier } & : & 1.0000 \\ \text { Dilution } & : & 1.0000\end{array}$

Use Multiplier \& Dilution Factor with ISTDs

Signal 1: DAD1 A, Sig=220,4 Ref=360,100

\begin{tabular}{|c|c|c|c|c|c|c|}
\hline $\begin{array}{c}\text { Peak } \\
\#\end{array}$ & $\begin{array}{c}\text { RetTime } \\
\text { [min] }\end{array}$ & Type & $\begin{array}{l}\text { Width } \\
\text { [min] }\end{array}$ & $\begin{array}{c}\text { Area } \\
{\left[\mathrm{mAU}^{*} \mathrm{~s}\right]}\end{array}$ & $\begin{array}{l}\text { Height } \\
{[\mathrm{mAU}]}\end{array}$ & $\begin{array}{c}\text { Area } \\
\%\end{array}$ \\
\hline 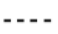 & & & & 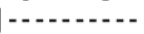 & & $-\ldots$ \\
\hline 1 & 17.265 & BB & 0.5831 & $2.37425 \mathrm{e} 4$ & 636.63153 & 45.2456 \\
\hline 2 & 20.365 & BB & 0.6476 & $2.38701 \mathrm{e} 4$ & 573.49780 & 45.4887 \\
\hline 3 & 32.018 & BB & 0.8744 & 2437.18799 & 40.25427 & 4.6445 \\
\hline
\end{tabular}

1260-DAD 9/3/2021 15:19:50 SYSTEM

\begin{tabular}{|c|c|c|c|c|c|}
\hline $\begin{array}{c}\text { Peak } \\
\#\end{array}$ & $\begin{array}{l}\text { RetTime Type } \\
\text { [min] }\end{array}$ & $\begin{array}{l}\text { Width } \\
\text { [min] }\end{array}$ & $\begin{array}{c}\text { Area } \\
{\left[\mathrm{mAU}^{*} \mathrm{~s}\right]}\end{array}$ & $\begin{array}{l}\text { Height } \\
\text { [mAU] }\end{array}$ & $\begin{array}{c}\text { Area } \\
\%\end{array}$ \\
\hline 4 & $43.500 \mathrm{BB}$ & 1.0770 & 2424.93994 & 27.12485 & 4.6212 \\
\hline
\end{tabular}

Totals : $\quad 5.24747$ e4 1277.50846

$* * *$ End of Report *** 


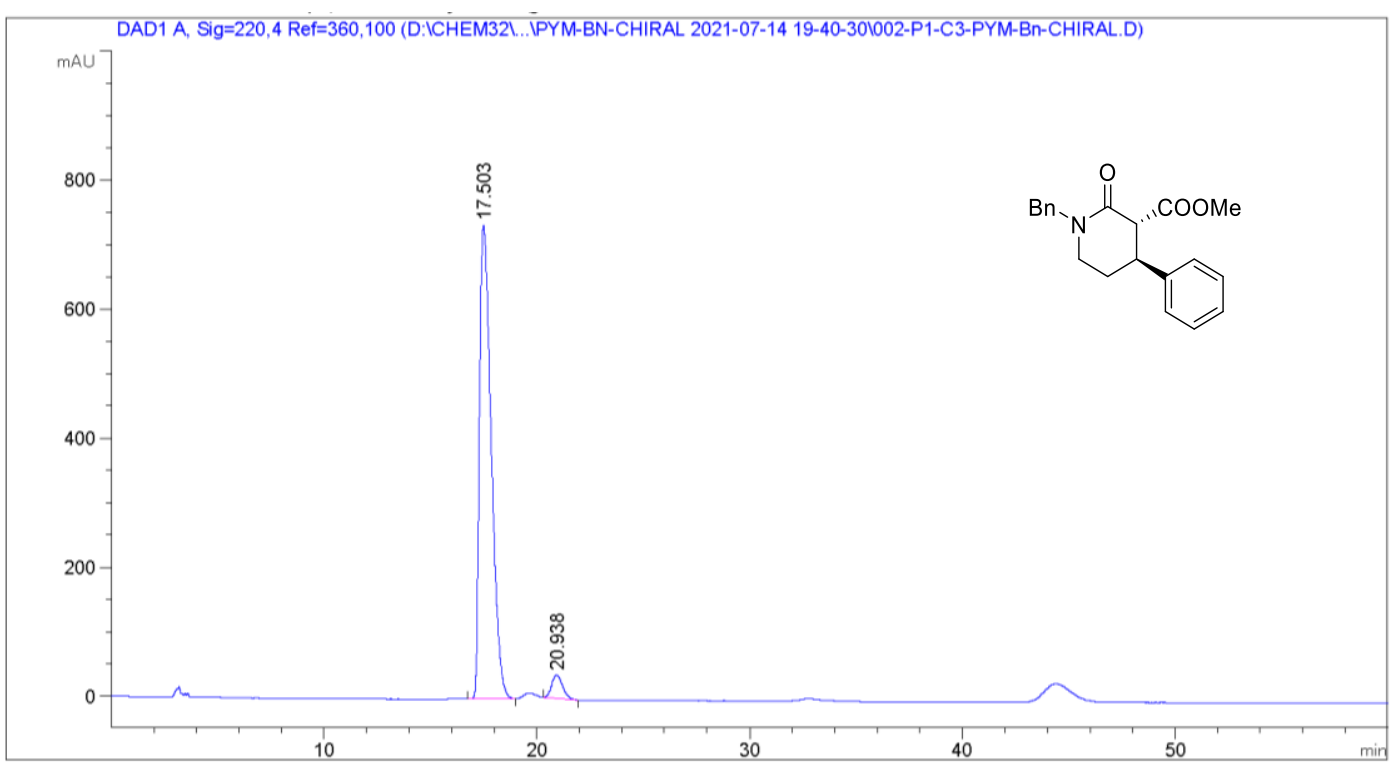

Area Percent Report

$\begin{array}{lll}\text { Sorted By } & : & \text { Signal } \\ \text { Multiplier } & : & 1.0000 \\ \text { Dilution } & : & 1.0000\end{array}$

Use Multiplier \& Dilution Factor with ISTDs

Signal 1: DAD1 A, Sig=220,4 Ref=360,100

\begin{tabular}{|c|c|c|c|c|c|}
\hline $\begin{array}{c}\text { Peak } \\
\#\end{array}$ & $\begin{array}{l}\text { RetTime Type } \\
\text { [min] }\end{array}$ & $\begin{array}{l}\text { Width } \\
\text { [min] }\end{array}$ & $\begin{array}{c}\text { Area } \\
{\left[\mathrm{mAU}^{*} \mathrm{~s}\right]}\end{array}$ & $\begin{array}{l}\text { Height } \\
\text { [mAU] }\end{array}$ & $\begin{array}{c}\text { Area } \\
\%\end{array}$ \\
\hline & & & | & 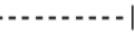 & $\mid$ \\
\hline 1 & $17.503 \mathrm{BB}$ & 0.5950 & $2.81376 \mathrm{e} 4$ & 734.46893 & 95.6445 \\
\hline 2 & $20.938 \mathrm{BB}$ & 0.5401 & 1281.33997 & 36.27766 & 4.3555 \\
\hline
\end{tabular}

$\begin{array}{lll}\text { Totals : } & 2.94189 \mathrm{e} 4 & 770.74659\end{array}$ 
Methyl (3R,4S)-1-benzyl-4-(4-methoxyphenyl)-2-oxopiperidine-3-carboxylate 2g

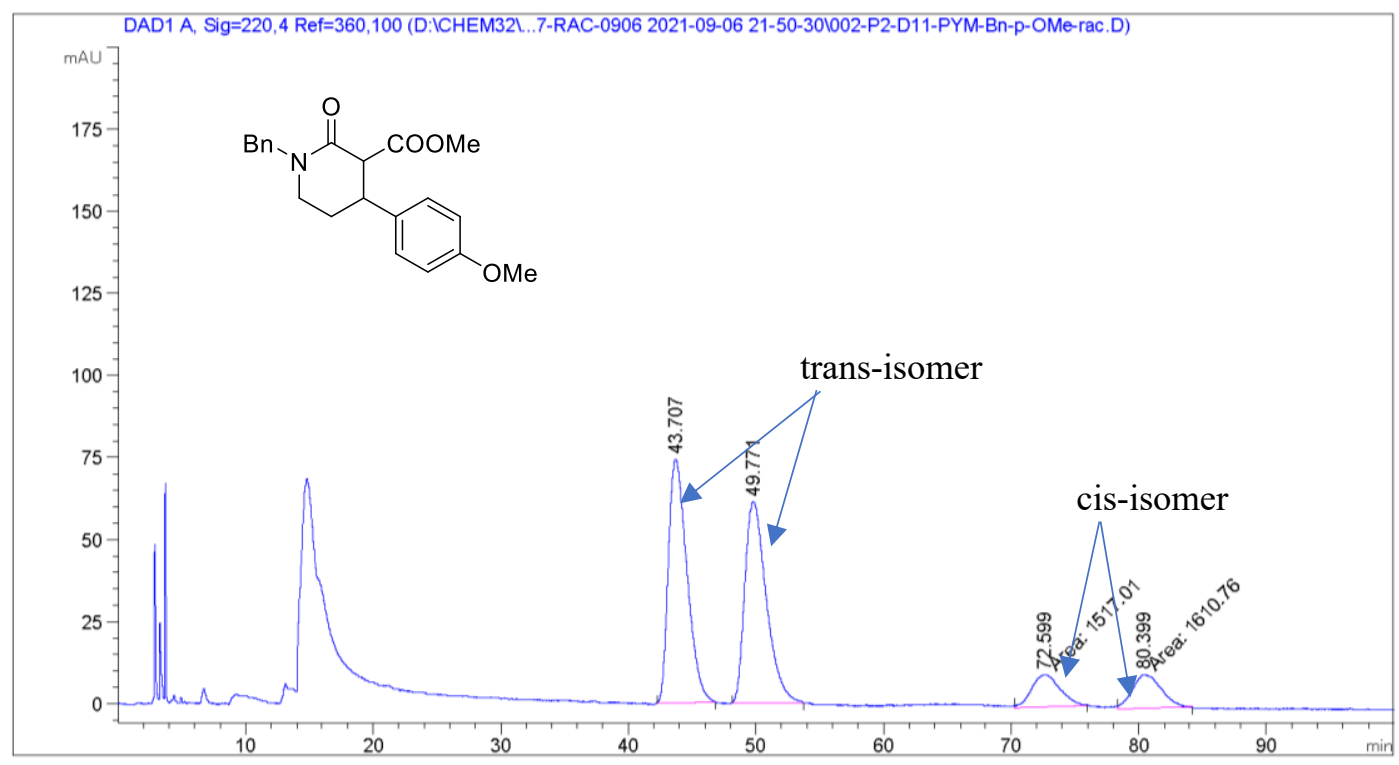

Area Percent Report

\begin{tabular}{|c|c|c|c|c|}
\hline Sorted By & : & Signal & & \\
\hline Multiplier & : & 1.0000 & & \\
\hline Dilution & : & 1.0000 & & \\
\hline \multicolumn{5}{|c|}{ Use Multiplier \& Dilution Factor with ISTDs } \\
\hline \multicolumn{5}{|c|}{ Signal 1: DAD1 A, Sig $=220,4 \operatorname{Re} f=360,100$} \\
\hline $\begin{array}{l}\text { Peak RetTime Type } \\
\# \quad[\mathrm{~min}]\end{array}$ & $\begin{array}{l}\text { Width } \\
\text { [min] }\end{array}$ & $\begin{array}{c}\text { Area } \\
{[\mathrm{mAU} * \mathrm{~s}]}\end{array}$ & $\begin{array}{l}\text { Height } \\
{[\mathrm{mAU}]}\end{array}$ & $\begin{array}{c}\text { Area } \\
\%\end{array}$ \\
\hline 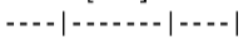 & $-\ldots-1$ & $|-2-2-1|$ & | & 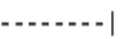 \\
\hline $143.707 \mathrm{BB}$ & 1.1905 & 7466.68359 & 74.16572 & 41.9920 \\
\hline $249.771 \mathrm{BB}$ & 1.3785 & 7186.76807 & 61.35910 & 40.4177 \\
\hline 372.599 MM & 2.5623 & 1517.01135 & 9.86734 & 8.5315 \\
\hline 480.399 MM & 2.6148 & 1610.75989 & 10.26704 & 9.0588 \\
\hline
\end{tabular}

1260-DAD 9/22/2021 18:59:01 SYSTEM 


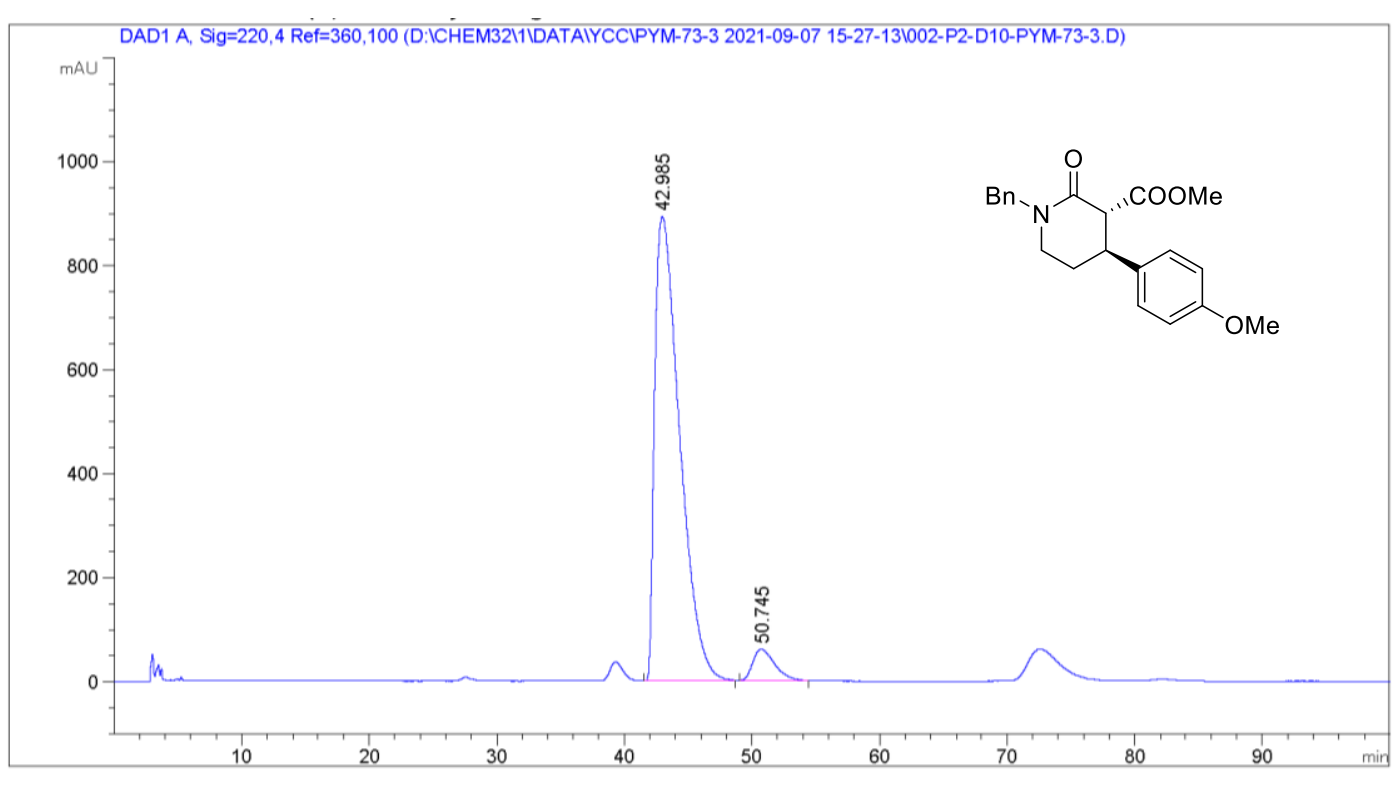

Area Percent Report

$\begin{array}{lll}\text { Sorted By } & : & \text { Signal } \\ \text { Multiplier } & : & 1.0000 \\ \text { Dilution } & : & 1.0000\end{array}$

Use Multiplier \& Dilution Factor with ISTDs

Signal 1: DAD1 A, Sig=220,4 Ref $=360,100$

\begin{tabular}{cccccc}
$\begin{array}{c}\text { Peak RetTime Type } \\
\text { Width }\end{array}\left[\begin{array}{c}\text { Area } \\
{[\text { min] }}\end{array}\right.$ & $\begin{array}{c}\text { Height } \\
{[\text { mAU*s] }}\end{array}$ & $\begin{array}{c}\text { Area } \\
\text { [mAU] }\end{array}$ & $\%$ \\
\hline 1 & 42.985 BB & 1.5949 & $1.20796 e 5$ & 893.72076 & 94.4020 \\
2 & 50.745 BB & 1.4039 & 7163.20410 & 60.12582 & 5.5980 \\
& & & & \\
Totals : & & $1.27959 e 5$ & 953.84659
\end{tabular}




\section{Methyl (3R,4S)-1-benzyl-4-(3-methoxyphenyl)-2-oxopiperidine-3-carboxylate $2 \mathrm{~h}$}

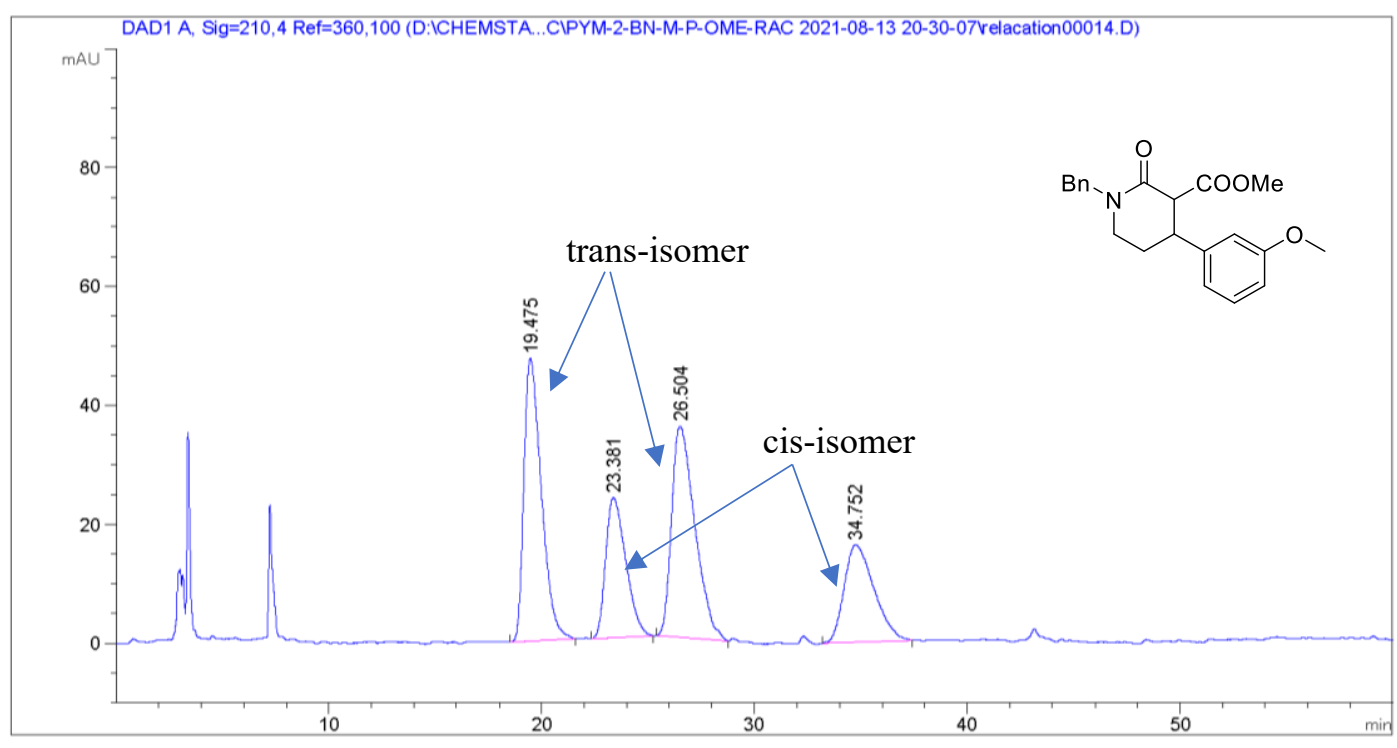

Area Percent Report

$\begin{array}{lcc}\text { Sorted By } & : & \text { Signal } \\ \text { Multiplier } & : & 1.0000 \\ \text { Dilution } & : & 1.0000 \\ \text { Do not use Multiplier } & \& & \text { Dilution Factor with ISTDs }\end{array}$

Signal 1: DAD1 A, Sig=210,4 Ref $=360,100$

\begin{tabular}{cccccc}
$\begin{array}{c}\text { Peak RetTime Type } \\
\text { \# }\end{array}$ [min] & $\begin{array}{c}\text { Width } \\
{[\text { min] }}\end{array}$ & $\begin{array}{c}\text { Area } \\
{[\text { mAU*s] }}\end{array}$ & $\begin{array}{c}\text { Height } \\
{[\mathrm{mAU}]}\end{array}$ & $\begin{array}{c}\text { Area } \\
\%\end{array}$ \\
\hline 1 & 19.475 BB & 0.8332 & 2700.64233 & 47.51093 & 31.6787 \\
2 & 23.381 BB & 0.9182 & 1548.46167 & 23.57762 & 18.1635 \\
3 & 26.504 BB & 1.0249 & 2678.89258 & 35.47856 & 31.4235
\end{tabular}

\begin{tabular}{|c|c|c|c|c|c|}
\hline $\begin{array}{c}\text { Peak } \\
\#\end{array}$ & $\begin{array}{l}\text { RetTime Type } \\
\text { [min] }\end{array}$ & $\begin{array}{l}\text { Width } \\
\text { [min] }\end{array}$ & $\begin{array}{c}\text { Area } \\
{\left[\mathrm{mAU}^{*} \mathrm{~s}\right]}\end{array}$ & $\begin{array}{l}\text { Height } \\
{[\mathrm{mAU}]}\end{array}$ & $\begin{array}{c}\text { Area } \\
\%\end{array}$ \\
\hline 4 & 34.752 BB & 1.1714 & 1597.11633 & 16.38155 & 18.7343 \\
\hline
\end{tabular}

Totals : $\quad 8525.11292 \quad 122.94865$ 


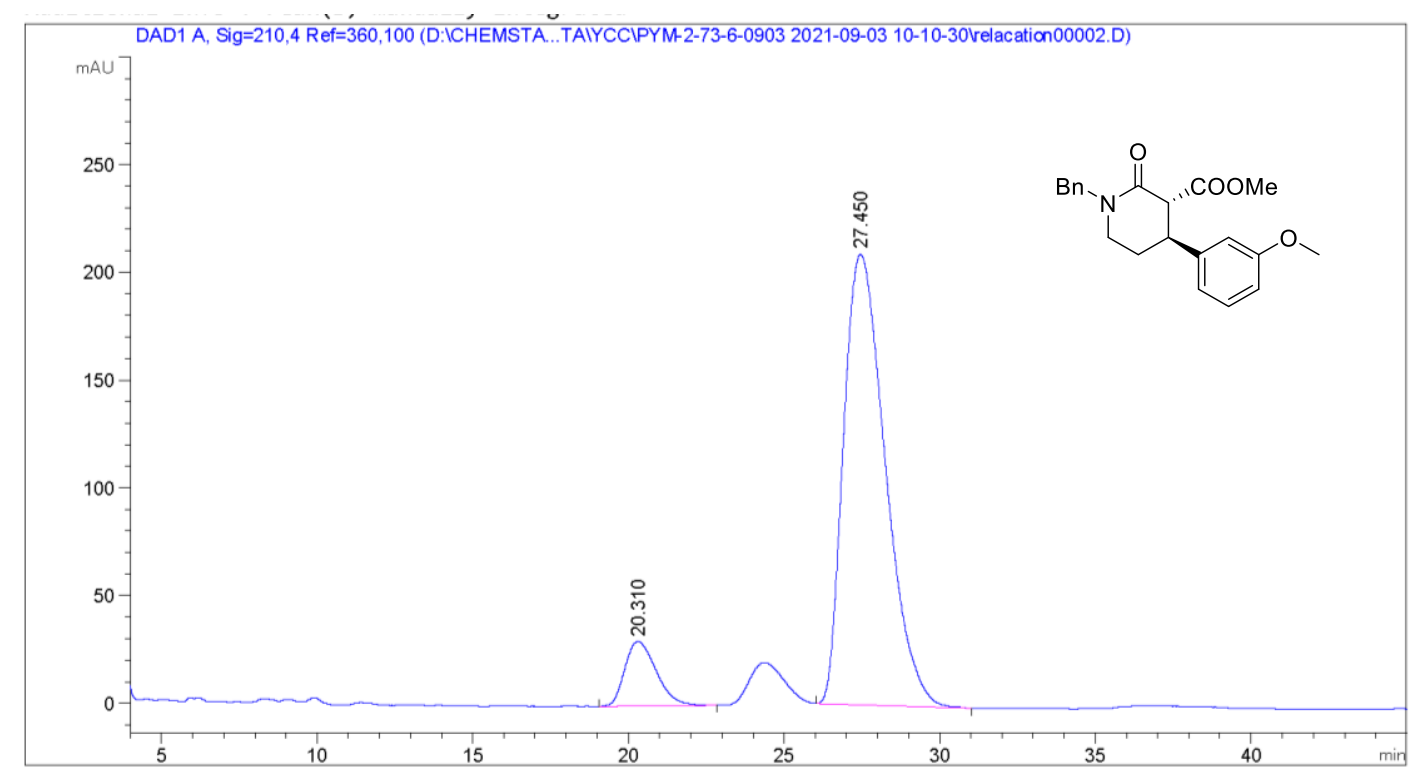

Area Percent Report

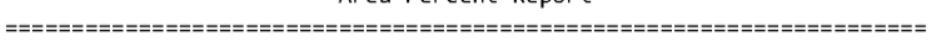

$\begin{array}{lll}\text { Sorted By } & : & \text { Signal } \\ \text { Multiplier } & : & 1.0000 \\ \text { Dilution } & : & 1.0000\end{array}$

Use Multiplier \& Dilution Factor with ISTDs

Signal 1: DAD1 A, Sig=210,4 Ref=360,100

\begin{tabular}{|c|c|c|c|c|c|c|}
\hline $\begin{array}{c}\text { Peak } \\
\#\end{array}$ & $\begin{array}{c}\text { RetTime } \\
\text { [min] }\end{array}$ & Type & $\begin{array}{l}\text { Width } \\
\text { [min] }\end{array}$ & $\begin{array}{c}\text { Area } \\
{\left[\mathrm{mAU}^{*} \mathrm{~s}\right]}\end{array}$ & $\begin{array}{l}\text { Height } \\
\text { [mAU] }\end{array}$ & $\begin{array}{c}\text { Area } \\
\%\end{array}$ \\
\hline- & & & & 5 & 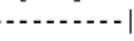 & $-\ldots$ \\
\hline 1 & 20.310 & BB & 0.9648 & 2105.09058 & 29.99569 & 9.7632 \\
\hline 2 & 27.450 & BB & 1.4040 & $1.94564 \mathrm{e} 4$ & 209.01627 & 90.2368 \\
\hline
\end{tabular}




\section{Methyl (3R,4S)-1-benzyl-4-(4-fluorophenyl)-2-oxopiperidine-3-carboxylate 2i}

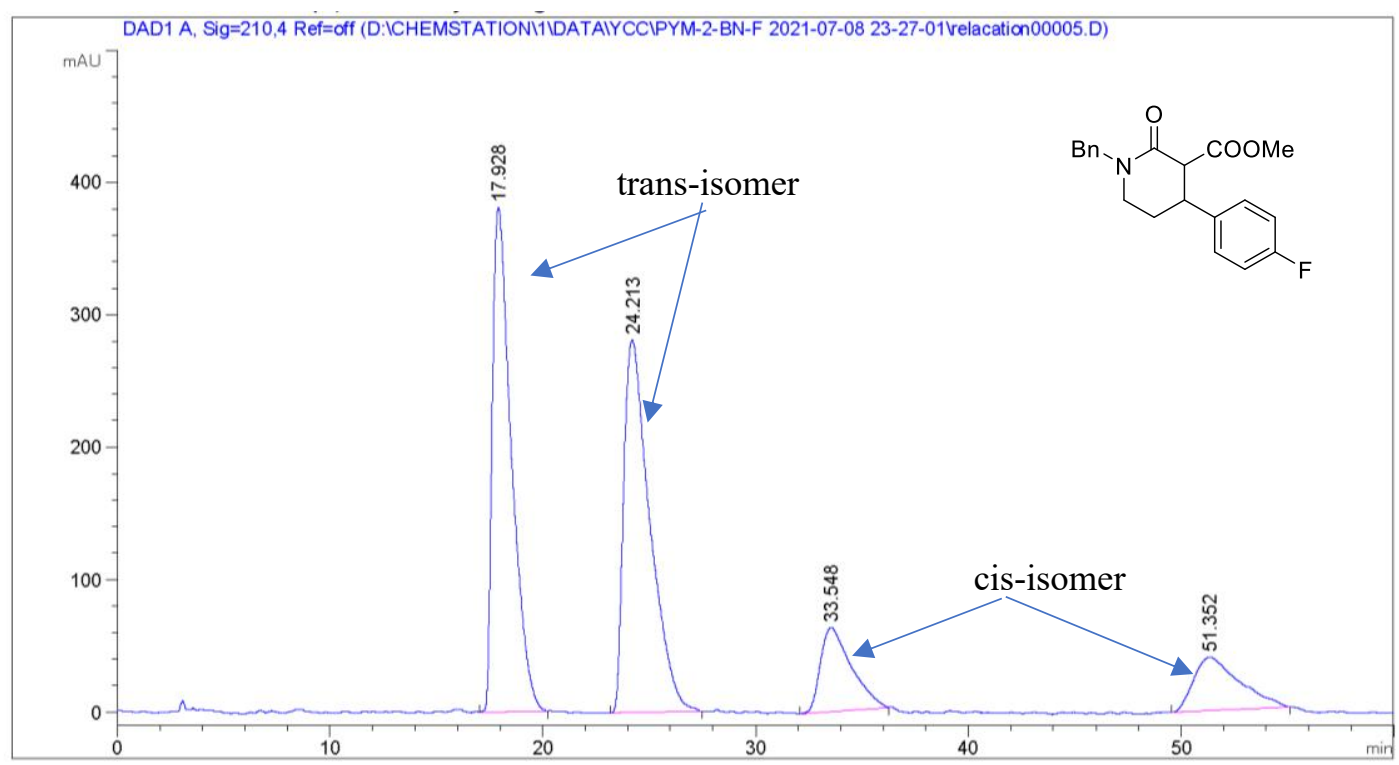

\section{Area Percent Report}

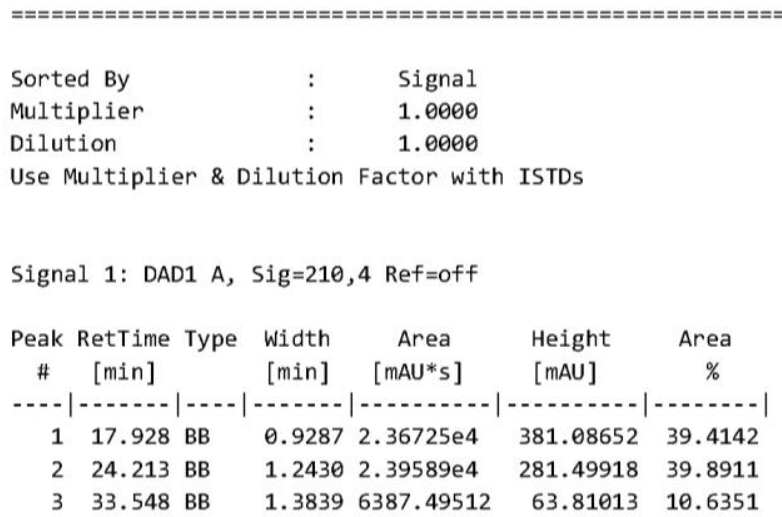

Data File D: \CHEMSTATION \1\DATA \YCC\PYM-2-BN-F 2021-07-08 23-27-01\relacatione0005.D Sample Name: pym-2-Bn-F-rac

\begin{tabular}{|c|c|c|c|c|c|}
\hline $\begin{array}{c}\text { Peak } \\
\#\end{array}$ & $\begin{array}{l}\text { RetTime Type } \\
\text { [min] }\end{array}$ & $\begin{array}{l}\text { Width } \\
\text { [min] }\end{array}$ & $\begin{array}{c}\text { Area } \\
{[\mathrm{mAU} * \mathrm{~s}]}\end{array}$ & $\begin{array}{l}\text { Height } \\
{[\mathrm{mAU}]}\end{array}$ & $\begin{array}{c}\text { Area } \\
\%\end{array}$ \\
\hline
\end{tabular}

Totals : $\quad 6.00608 \mathrm{e} 4 \quad 766.79721$ 


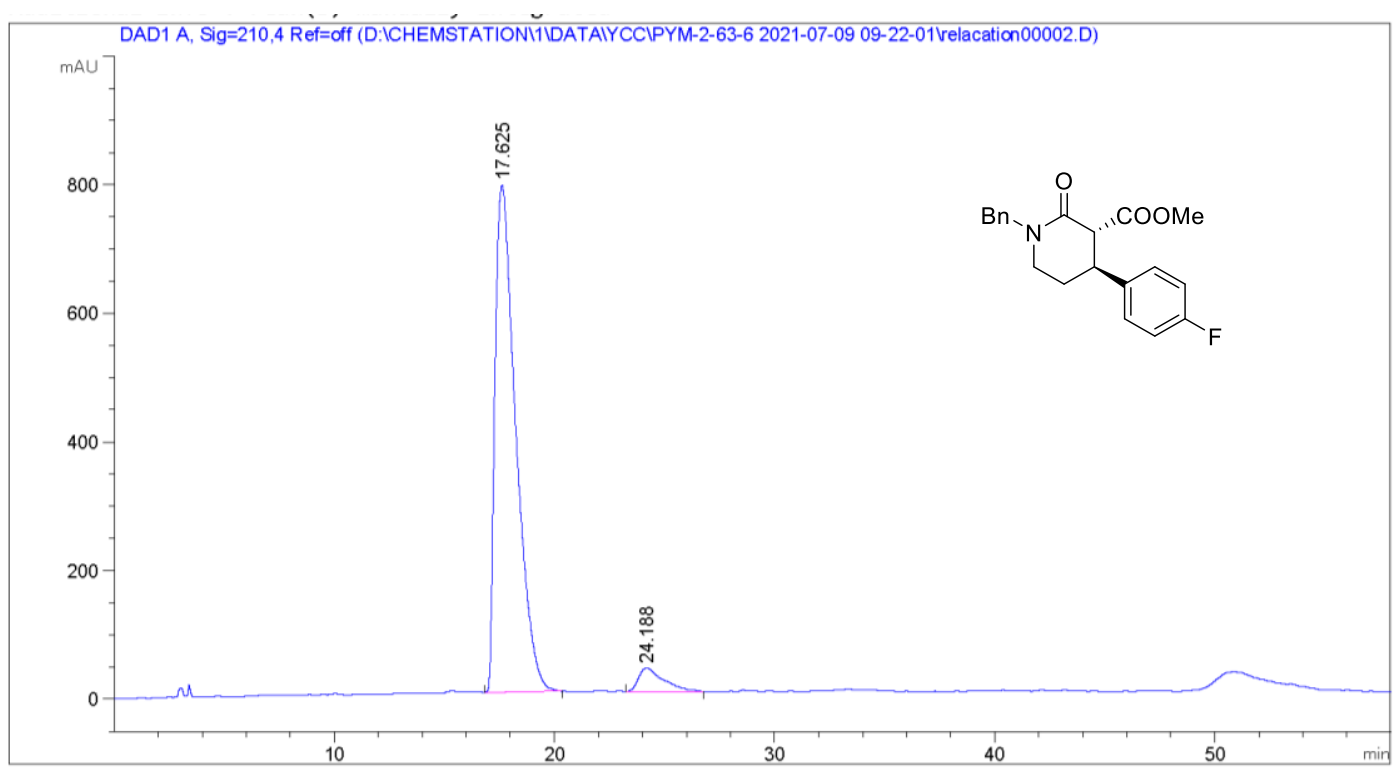

Area Percent Report

\begin{tabular}{|c|c|c|c|c|}
\hline Sorted By & : & Signal & & \\
\hline Multiplier & : & 1.0000 & & \\
\hline Dilution & : & 1.0000 & & \\
\hline \multicolumn{5}{|c|}{ Use Multiplier \& Dilution Factor with ISTDs } \\
\hline \multicolumn{5}{|c|}{ Signal 1: DAD1 A, Sig=210,4 Ref=off } \\
\hline $\begin{array}{l}\text { Peak RetTime Type } \\
\# \text { [min] }\end{array}$ & $\begin{array}{l}\text { Width } \\
\text { [min] }\end{array}$ & $\begin{array}{c}\text { Area } \\
{[\mathrm{mAU} * \mathrm{~s}]}\end{array}$ & $\begin{array}{l}\text { Height } \\
\text { [mAU] }\end{array}$ & $\begin{array}{c}\text { Area } \\
\%\end{array}$ \\
\hline 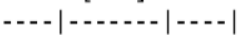 & $|--\ldots|$ & $|--2-1|$ & 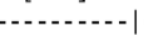 & 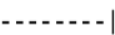 \\
\hline 117.625 BB & 0.9835 & $5.14618 \mathrm{e} 4$ & 788.47815 & 94.8622 \\
\hline $224.188 \mathrm{BB}$ & 1.0714 & 2787.22095 & 36.31534 & 5.1378 \\
\hline
\end{tabular}

LC 9/3/2021 2:58:33 PM SYSTEM 
ent-2i

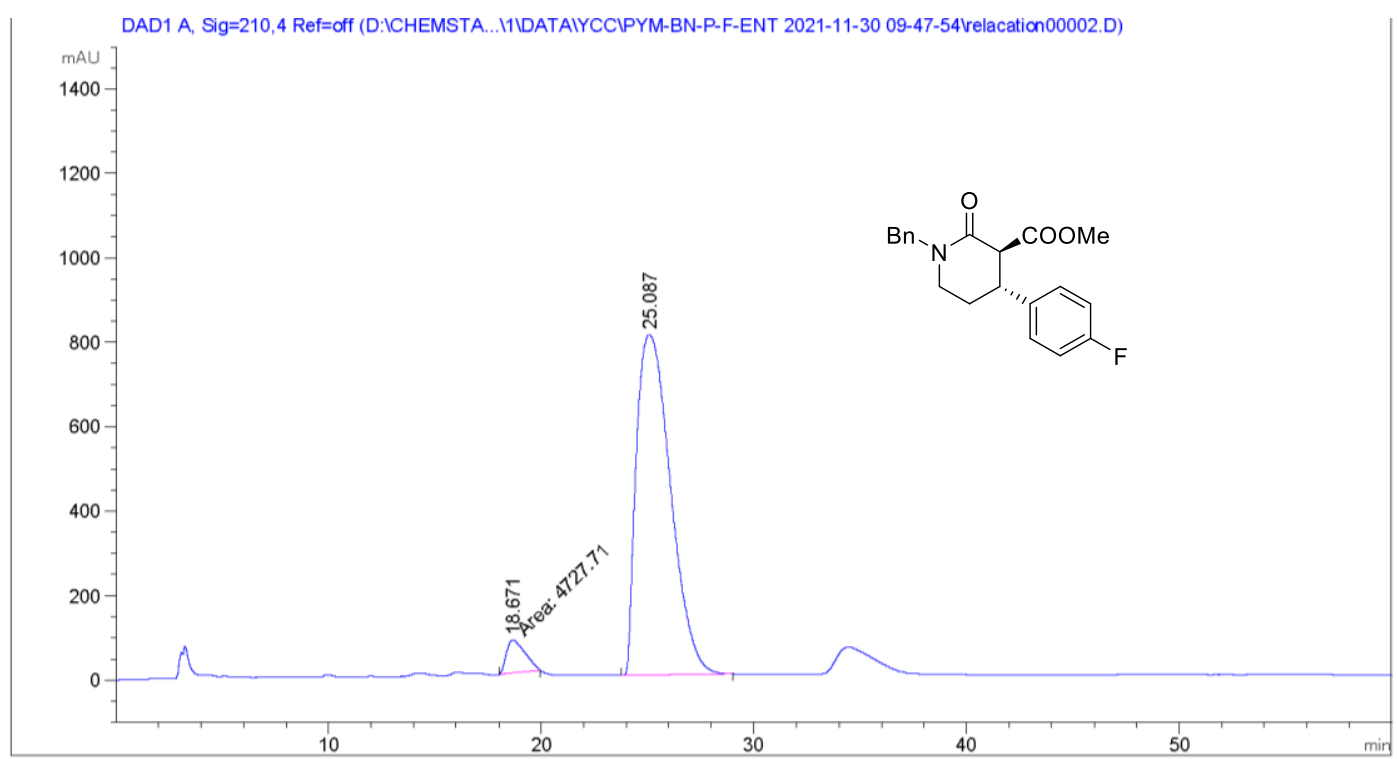

Area Percent Report

Sorted By : : Signal

Multiplier : $\quad 1.0000$

Dilution : 1.0000

Use Multiplier \& Dilution Factor with ISTDs

Signal 1: DAD1 A, Sig=210, 4 Ref=off

\begin{tabular}{|c|c|c|c|c|c|c|}
\hline $\begin{array}{c}\text { Peak } \\
\#\end{array}$ & $\begin{array}{c}\text { RetTime } \\
\text { [min] }\end{array}$ & Type & $\begin{array}{l}\text { Width } \\
\text { [min] }\end{array}$ & $\begin{array}{c}\text { Area } \\
{\left[\mathrm{mAU}^{*} \mathrm{~s}\right]}\end{array}$ & $\begin{array}{l}\text { Height } \\
\text { [mAU] }\end{array}$ & $\begin{array}{c}\text { Area } \\
\%\end{array}$ \\
\hline$=$ & & & & - & - & |- - - - - - - \\
\hline 1 & 18.671 & MM & 1.0114 & 4727.71289 & 77.90345 & 5.0057 \\
\hline 2 & 25.087 & BB & 1.3784 & $8.97189 \mathrm{e} 4$ & 806.17883 & 94.9943 \\
\hline
\end{tabular}

LC 11/30/2021 3:16:08 PM SYSTEM

Page 1 of 2 


\section{Methyl (3R,4S)-1-benzyl-4-(3-fluorophenyl)-2-oxopiperidine-3-carboxylate $2 \mathrm{j}$}

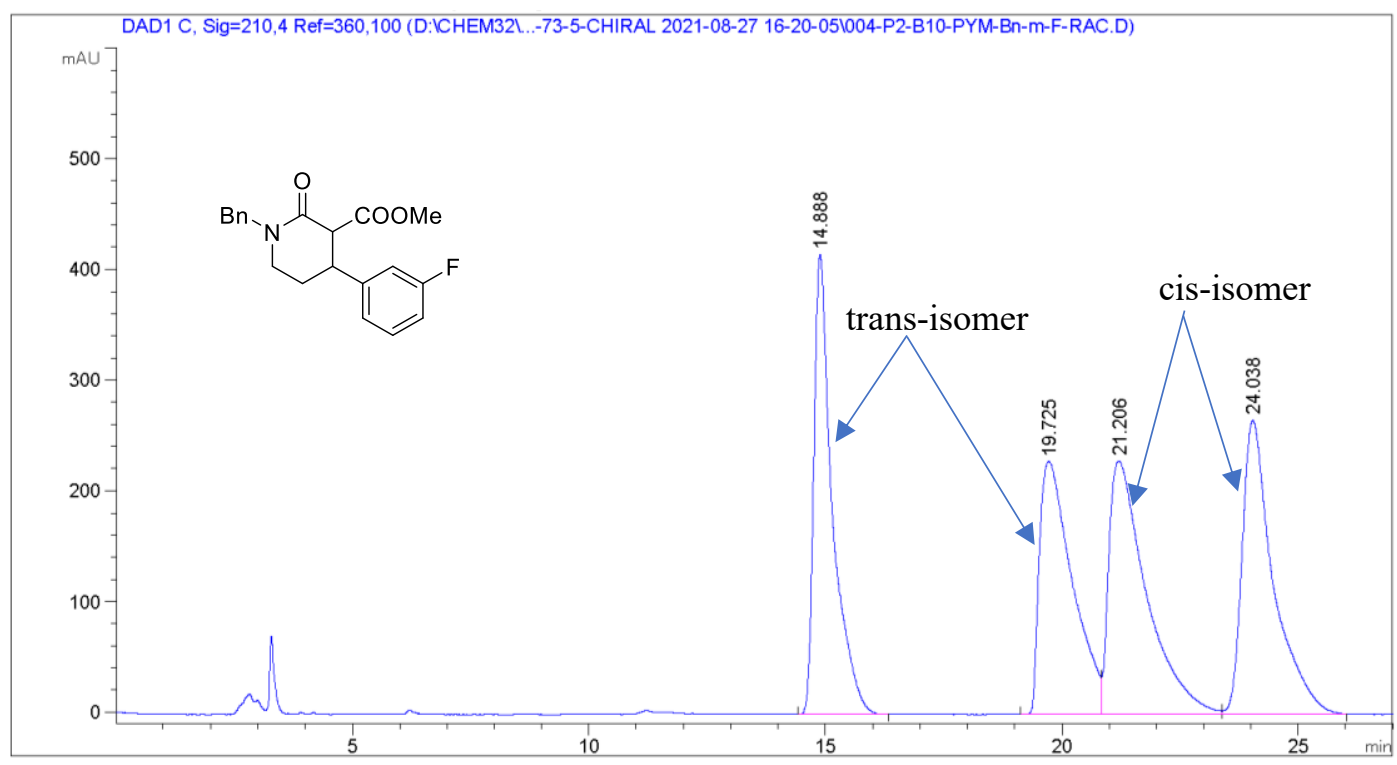

Area Percent Report

\begin{tabular}{|c|c|c|c|c|}
\hline Sorted By & : & Signal & & \\
\hline Multiplier & : & 1.0000 & & \\
\hline Dilution & : & 1.0000 & & \\
\hline \multicolumn{5}{|c|}{ Use Multiplier \& Dilution Factor with ISTDs } \\
\hline \multicolumn{5}{|c|}{ Signal 1: DAD1 C, Sig=210,4 $\operatorname{Re} f=360,100$} \\
\hline $\begin{array}{l}\text { Peak RetTime Type } \\
\# \quad[\mathrm{~min}]\end{array}$ & $\begin{array}{l}\text { Width } \\
\text { [min] }\end{array}$ & $\begin{array}{c}\text { Area } \\
{\left[\mathrm{mAU}^{*} \mathrm{~s}\right]}\end{array}$ & $\begin{array}{l}\text { Height } \\
\text { [mAU] }\end{array}$ & $\begin{array}{c}\text { Area } \\
\%\end{array}$ \\
\hline 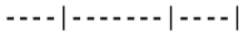 & 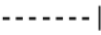 & 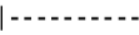 & 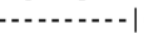 & 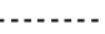 \\
\hline 114.888 BB & 0.3899 & $1.13210 \mathrm{e} 4$ & 415.48145 & 24.0178 \\
\hline $219.725 \mathrm{BV}$ & 0.6627 & $1.10221 \mathrm{e} 4$ & 228.50728 & 23.3836 \\
\hline $21.206 \mathrm{WV}$ & 0.7789 & $1.26257 \mathrm{e} 4$ & 229.11896 & 26.7857 \\
\hline $424.038 \mathrm{VB}$ & 0.6545 & $1.21672 \mathrm{e} 4$ & 265.56195 & 25.8129 \\
\hline
\end{tabular}




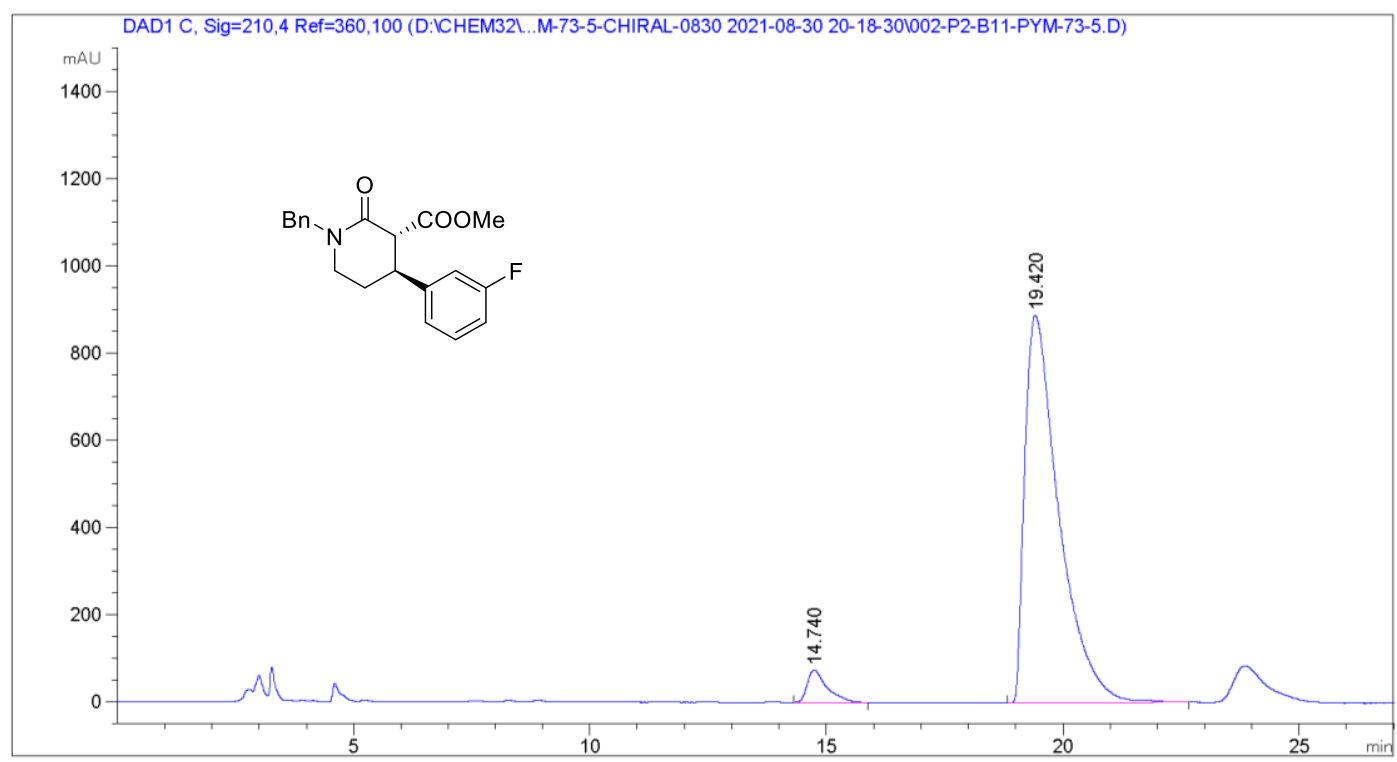

Area Percent Report

$\begin{array}{lll}\text { Sorted By } & : & \text { Signal } \\ \text { Multiplier } & : & 1.0000 \\ \text { Dilution } & : & 1.0000\end{array}$

Use Multiplier \& Dilution Factor with ISTDs

Signal 1: DAD1 C, Sig $=210,4$ Ref $=360,100$

\begin{tabular}{|c|c|c|c|c|c|c|}
\hline $\begin{array}{c}\text { Peak } \\
\#\end{array}$ & $\begin{array}{c}\text { RetTime } \\
\text { [min] }\end{array}$ & Type & $\begin{array}{l}\text { Width } \\
\text { [min] }\end{array}$ & $\begin{array}{c}\text { Area } \\
{[\mathrm{mAU} * \mathrm{~s}]}\end{array}$ & $\begin{array}{l}\text { Height } \\
{[\mathrm{mAU}]}\end{array}$ & $\begin{array}{c}\text { Area } \\
\%\end{array}$ \\
\hline$\ldots$ & |.... & $|---|$ & 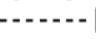 & |- & | & | \\
\hline 1 & 14.740 & BB & 0.4021 & 2172.56348 & 74.96795 & 4.6459 \\
\hline 2 & 19.420 & BV $R$ & 0.7315 & $4.45905 \mathrm{e} 4$ & 888.57080 & 95.3541 \\
\hline Tota & 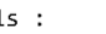 & & & $4.67631 \mathrm{e} 4$ & 963.53875 & \\
\hline
\end{tabular}




\section{Methyl (3R,4S)-1-benzyl-4-(3-bromophenyl)-2-oxopiperidine-3-carboxylate 2k}

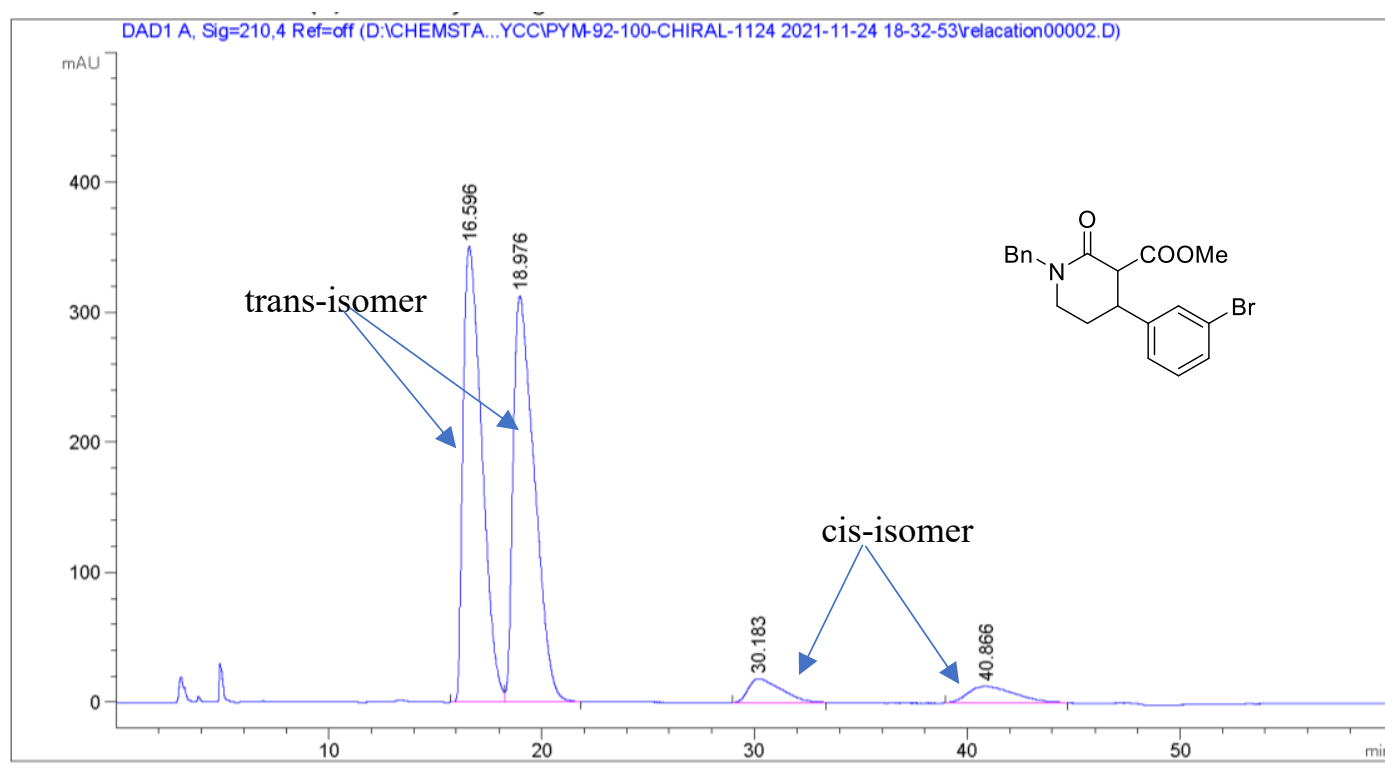

Area Percent Report

$\begin{array}{lll}\text { Sorted By } & : & \text { Signal } \\ \text { Multiplier } & : & 1.0000 \\ \text { Dilution } & : & 1.0000\end{array}$

Use Multiplier \& Dilution Factor with ISTDs

Signal 1: DAD1 A, Sig=210,4 Ref=off

\begin{tabular}{|c|c|c|c|c|c|c|}
\hline $\begin{array}{c}\text { Peak } \\
\#\end{array}$ & $\begin{array}{c}\text { RetTime } \\
\text { [min] }\end{array}$ & Type & $\begin{array}{l}\text { Width } \\
\text { [min] }\end{array}$ & $\begin{array}{c}\text { Area } \\
{\left[\mathrm{mAU}^{*} \mathrm{~s}\right]}\end{array}$ & $\begin{array}{l}\text { Height } \\
\text { [mAU] }\end{array}$ & $\begin{array}{c}\text { Area } \\
\%\end{array}$ \\
\hline- & & & & - & . & - \\
\hline 1 & 16.596 & BV & 0.9226 & $2.15181 \mathrm{e} 4$ & 350.33344 & 45.7736 \\
\hline 2 & 18.976 & VB & 0.9841 & $2.16263 \mathrm{e} 4$ & 312.22311 & 46.0037 \\
\hline 3 & 30.183 & BB & 1.2500 & 1954.42322 & 18.47805 & 4.1575 \\
\hline
\end{tabular}

LC $11 / 24 / 20218: 54: 03$ PM SYSTEM

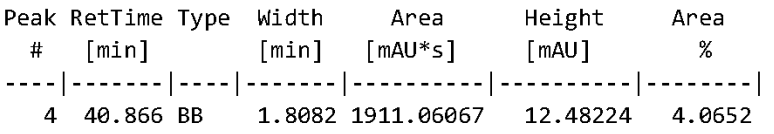

Totals : $\quad 4.70099 \mathrm{e} 4 \quad 693.51684$ 


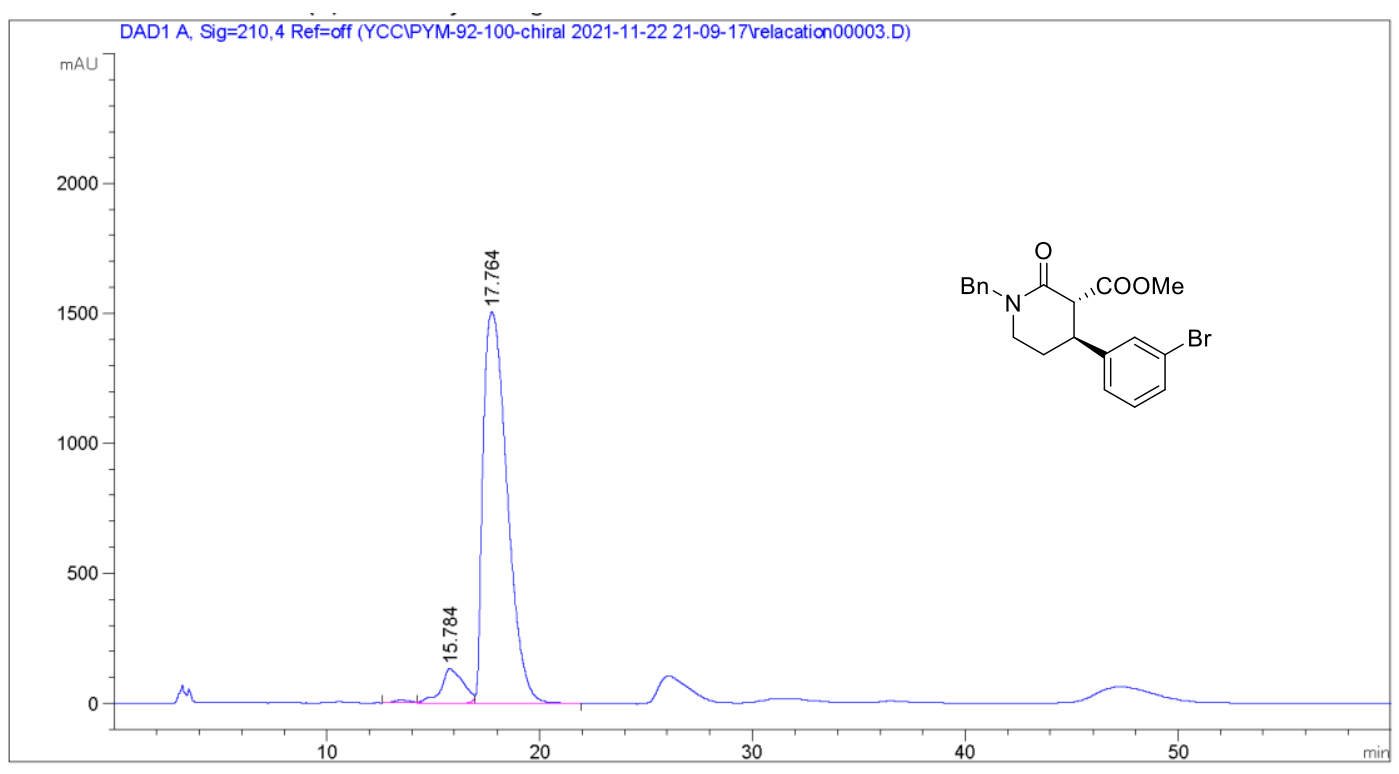

Area Percent Report

$\begin{array}{lll}\text { Sorted By } & : & \text { Signal } \\ \text { Multiplier } & : & 1.0000 \\ \text { Dilution } & : & 1.0000\end{array}$

Use Multiplier \& Dilution Factor with ISTDs

Signal 1: DAD1 A, Sig=210, 4 Ref=off

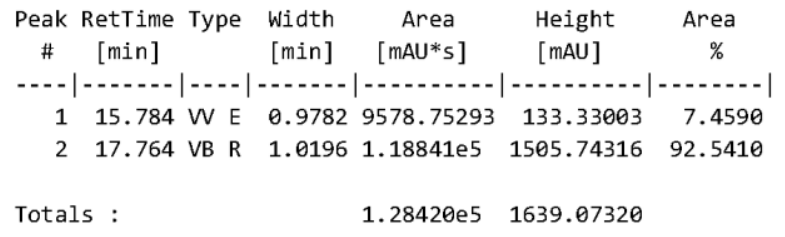


Methyl (3R,4S)-1-benzyl-2-oxo-4-(thiophen-2-yl)piperidine-3-carboxylate 21

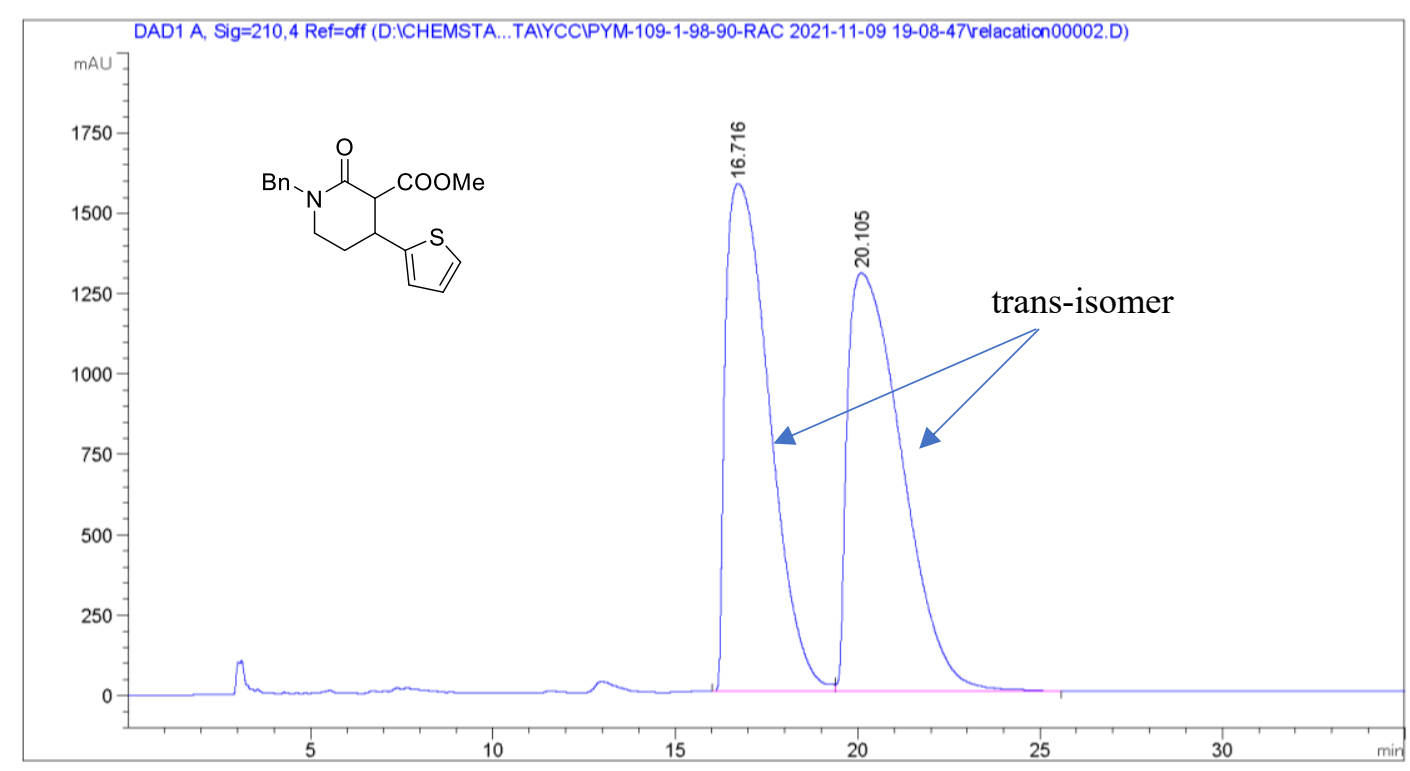

Area Percent Report

$\begin{array}{lll}\text { Sorted By } & : & \text { Signal } \\ \text { Multiplier } & : & 1.0000 \\ \text { Dilution } & : & 1.0000\end{array}$

Use Multiplier \& Dilution Factor with ISTDs

Signal 1: DAD1 A, Sig=210,4 Ref=off

\begin{tabular}{|c|c|c|c|c|c|}
\hline $\begin{array}{c}\text { Peak } \\
\#\end{array}$ & $\begin{array}{l}\text { RetTime Type } \\
\text { [min] }\end{array}$ & $\begin{array}{l}\text { Width } \\
\text { [min] }\end{array}$ & $\begin{array}{c}\text { Area } \\
{\left[\mathrm{mAU}^{*} \mathrm{~s}\right]}\end{array}$ & $\begin{array}{l}\text { Height } \\
{[\mathrm{mAU}]}\end{array}$ & $\begin{array}{c}\text { Area } \\
\%\end{array}$ \\
\hline & & & & & \\
\hline 1 & $16.716 \mathrm{BV}$ & 1.1036 & $1.31757 \mathrm{e} 5$ & 1578.76575 & 49.4577 \\
\hline 2 & $20.105 \mathrm{VB}$ & 1.3611 & $1.34646 \mathrm{e} 5$ & 1301.40649 & 50.5423 \\
\hline
\end{tabular}




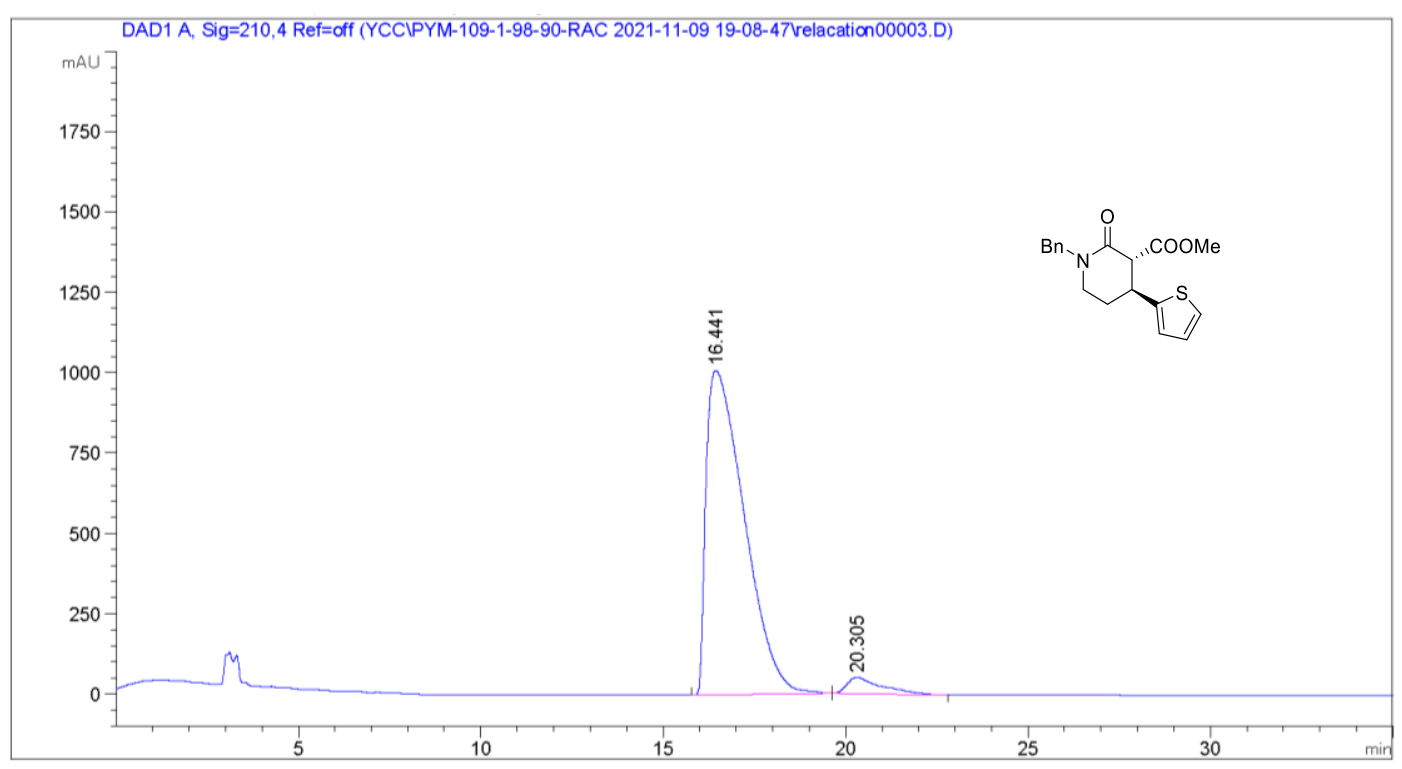

Area Percent Report

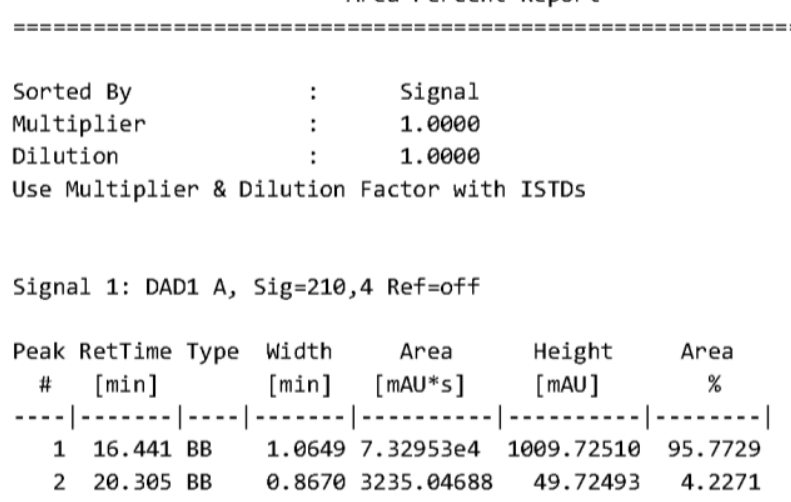


Methyl (3R,4S)-1-(4-methoxyphenyl)-4-methyl-2-oxopiperidine-3-carboxylate 2m

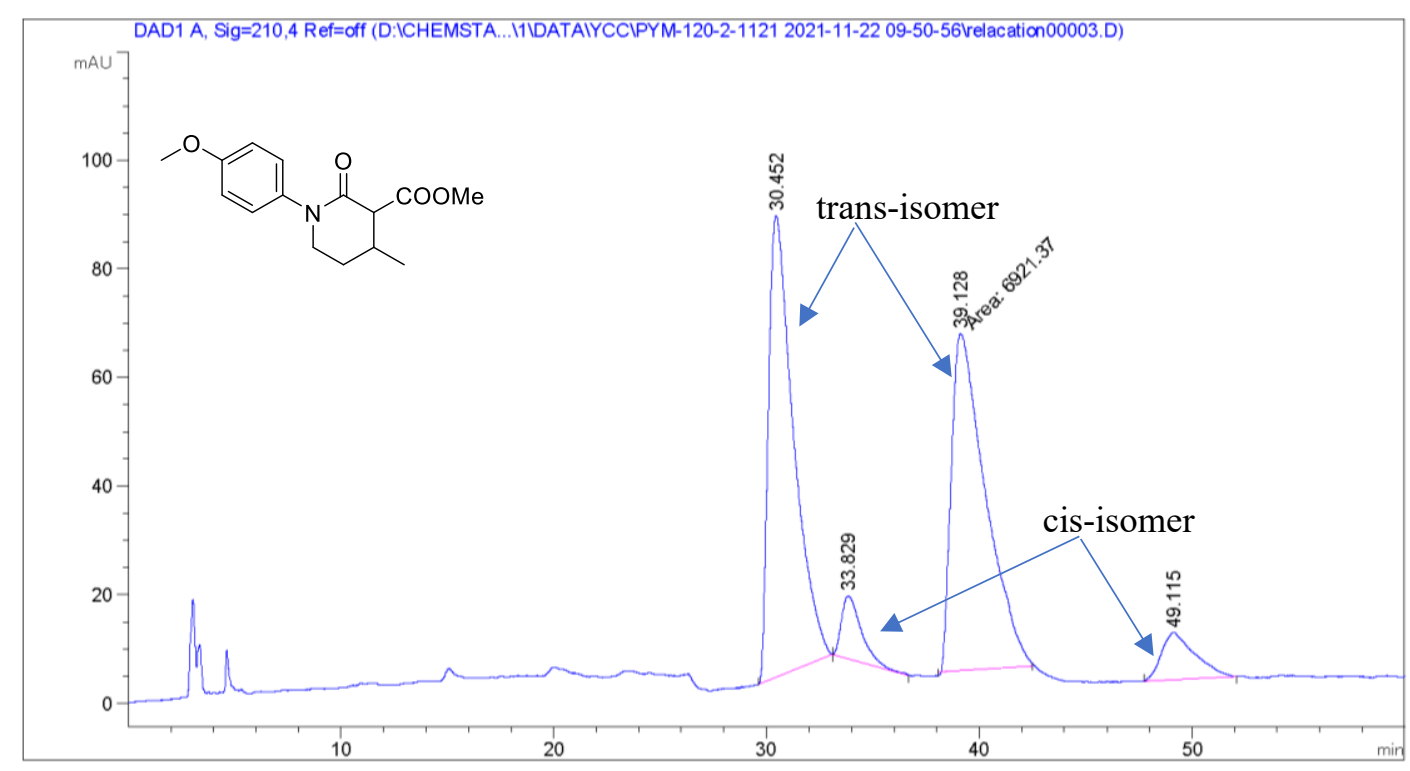

Area Percent Report

Sorted By : : Signal

Multiplier $\quad: \quad 1.0000$

Dilution : 1.0000

Use Multiplier \& Dilution Factor with ISTDs

Signal 1: DAD1 A, Sig=210,4 Ref=off

\begin{tabular}{|c|c|c|c|c|c|c|}
\hline $\begin{array}{c}\text { Peak } \\
\#\end{array}$ & $\begin{array}{c}\text { RetTime } \\
\text { [min] }\end{array}$ & Type & $\begin{array}{l}\text { Width } \\
\text { [min] }\end{array}$ & $\begin{array}{c}\text { Area } \\
{[\mathrm{mAU} * \mathrm{~s}]}\end{array}$ & $\begin{array}{l}\text { Height } \\
\text { [mAU] }\end{array}$ & $\begin{array}{c}\text { Area } \\
\%\end{array}$ \\
\hline - & & & & | & $2-2-2$ & 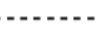 \\
\hline 1 & 30.452 & BB & 1.1463 & 6956.02734 & 85.04324 & 44.6760 \\
\hline 2 & 33.829 & BB & 0.7683 & 749.38409 & 11.59121 & 4.8130 \\
\hline 3 & 39.128 & MM & 1.8597 & 6921.37402 & 62.02935 & 44.4535 \\
\hline
\end{tabular}

LC 11/26/2021 9:14:03 PM SYSTEM

\begin{tabular}{|c|c|c|c|c|c|}
\hline $\begin{array}{c}\text { Peak } \\
\#\end{array}$ & $\begin{array}{l}\text { RetTime Type } \\
\text { [min] }\end{array}$ & $\begin{array}{l}\text { Width } \\
\text { [min] }\end{array}$ & $\begin{array}{c}\text { Area } \\
{[\mathrm{mAU} * \mathrm{~s}]}\end{array}$ & $\begin{array}{l}\text { Height } \\
{[\mathrm{mAU}]}\end{array}$ & $\begin{array}{c}\text { Area } \\
\%\end{array}$ \\
\hline$\ldots$ & $\mid$ & - & | & | & $-----\mid$ \\
\hline 4 & 49.115 BB & 1.2882 & 943.15002 & 8.63569 & 6.0575 \\
\hline Tota & & & $1.55699 \mathrm{e} 4$ & 167.2 & \\
\hline
\end{tabular}

*** End of Report **** 


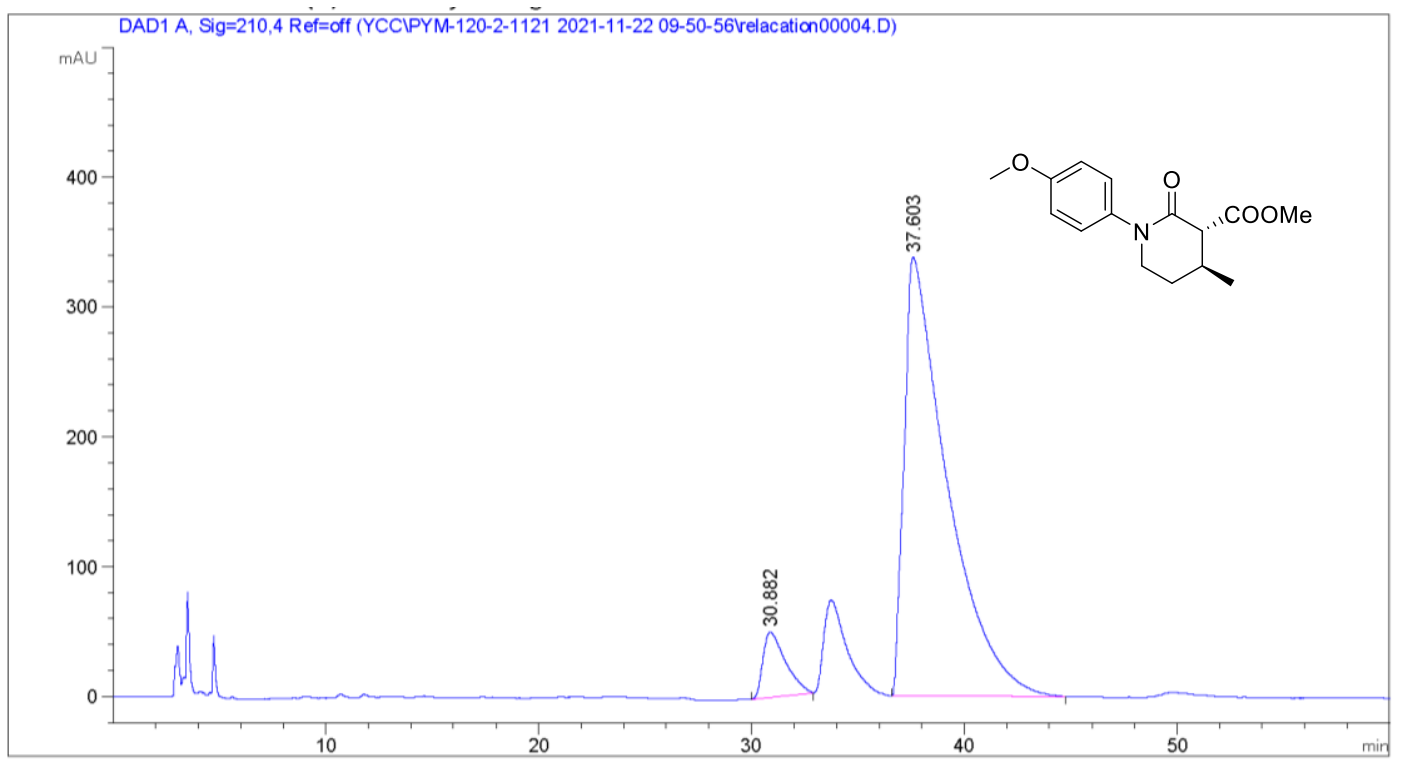

Area Percent Report

\begin{tabular}{|c|c|c|c|c|}
\hline Sorted By & : & Signal & & \\
\hline Multiplier & : & 1.0000 & & \\
\hline Dilution & : & 1.0000 & & \\
\hline \multicolumn{5}{|c|}{ Use Multiplier \& Dilution Factor with ISTDs } \\
\hline \multicolumn{5}{|c|}{ Signal 1: DAD1 A, Sig=210,4 Ref=off } \\
\hline $\begin{array}{l}\text { Peak RetTime Type } \\
\# \quad \text { [min] }\end{array}$ & $\begin{array}{l}\text { Width } \\
\text { [min] }\end{array}$ & $\begin{array}{c}\text { Area } \\
{\left[\mathrm{mAU}^{*} \mathrm{~s}\right]}\end{array}$ & $\begin{array}{l}\text { Height } \\
{[\mathrm{mAU}]}\end{array}$ & $\begin{array}{c}\text { Area } \\
\%\end{array}$ \\
\hline 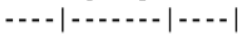 & 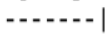 & $|-\cdots+\cdots|$ & | & $-\cdots-1$ \\
\hline 130.882 BB & 0.9920 & 3687.40161 & 50.13962 & 7.1954 \\
\hline $237.603 \mathrm{BB}$ & 1.8072 & $4.75596 \mathrm{e} 4$ & 337.67349 & 92.8046 \\
\hline
\end{tabular}

LC 11/26/2021 9:10:19 PM SYSTEM

Page 1 of 2 


\section{Methyl (3R,4R)-1-benzyl-4-cyclohexyl-2-oxopiperidine-3-carboxylate 2n}

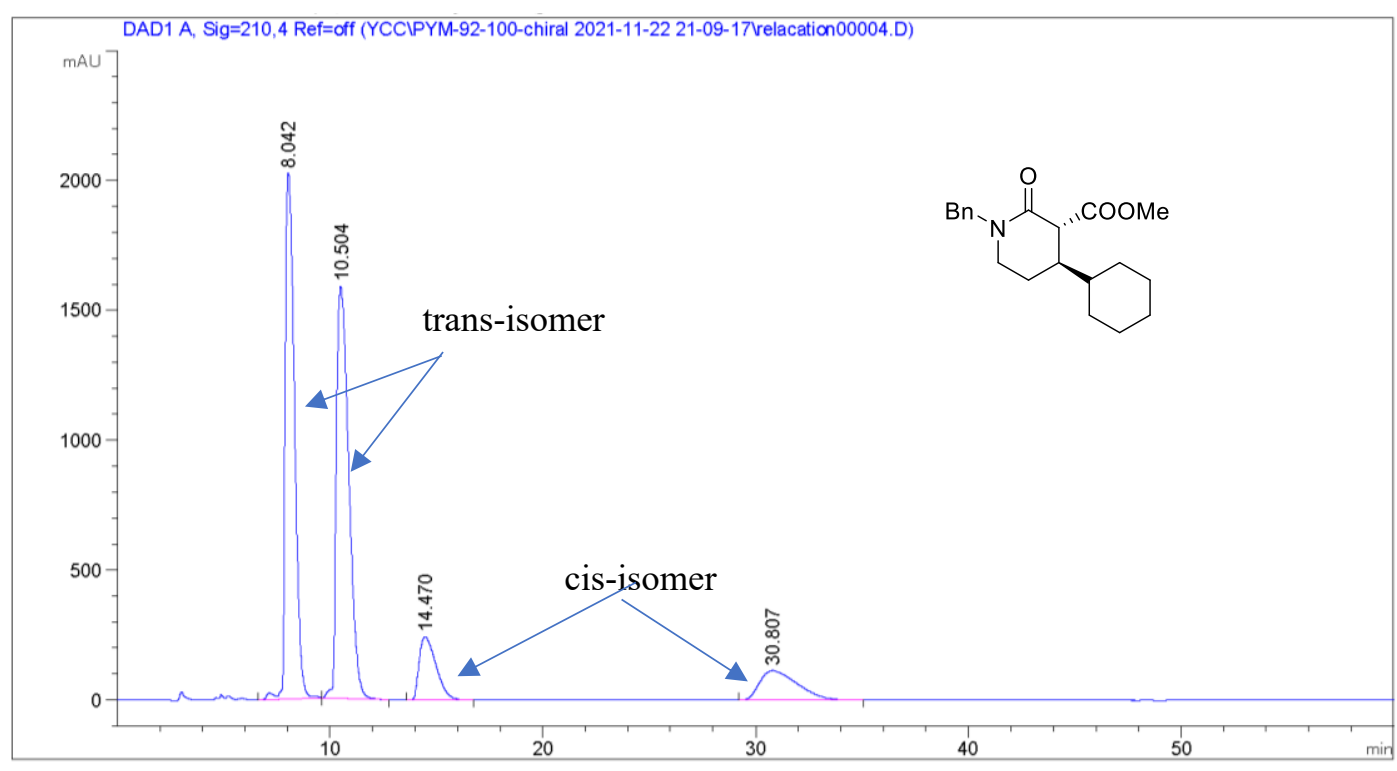

Area Percent Report

$\begin{array}{lll}\text { Sorted By } & : & \text { Signal } \\ \text { Multiplier } & : & 1.0000 \\ \text { Dilution } & : & 1.0000\end{array}$

Use Multiplier \& Dilution Factor with ISTDs

Signal 1: DAD1 A, Sig=210,4 Ref=off

\begin{tabular}{|c|c|c|c|c|c|c|}
\hline $\begin{array}{c}\text { Peak } \\
\#\end{array}$ & $\begin{array}{c}\text { RetTime } \\
\text { [min] }\end{array}$ & Type & $\begin{array}{l}\text { Width } \\
\text { [min] }\end{array}$ & $\begin{array}{c}\text { Area } \\
{\left[\mathrm{mAU}^{*} \mathrm{~s}\right]}\end{array}$ & $\begin{array}{l}\text { Height } \\
\text { [mAU] }\end{array}$ & $\begin{array}{c}\text { Area } \\
\%\end{array}$ \\
\hline & & & & 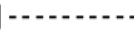 & 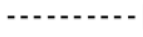 & 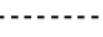 \\
\hline 1 & 8.042 & W R & 0.4849 & $6.16700 \mathrm{e} 4$ & 2021.64783 & 40.3465 \\
\hline 2 & 10.504 & BB & 0.6502 & $6.41931 \mathrm{e} 4$ & 1585.97290 & 41.9972 \\
\hline 3 & 14.470 & BB & 0.8363 & $1.32923 \mathrm{e} 4$ & 242.20250 & 8.6962 \\
\hline
\end{tabular}

LC 11/23/2021 2:41:33 PM SYSTEM

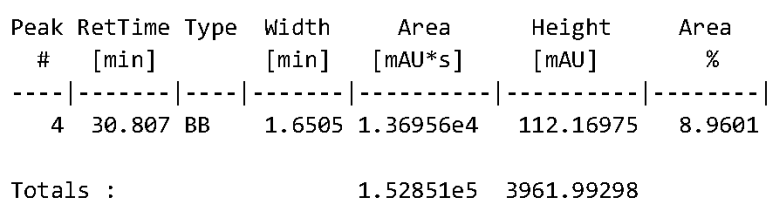




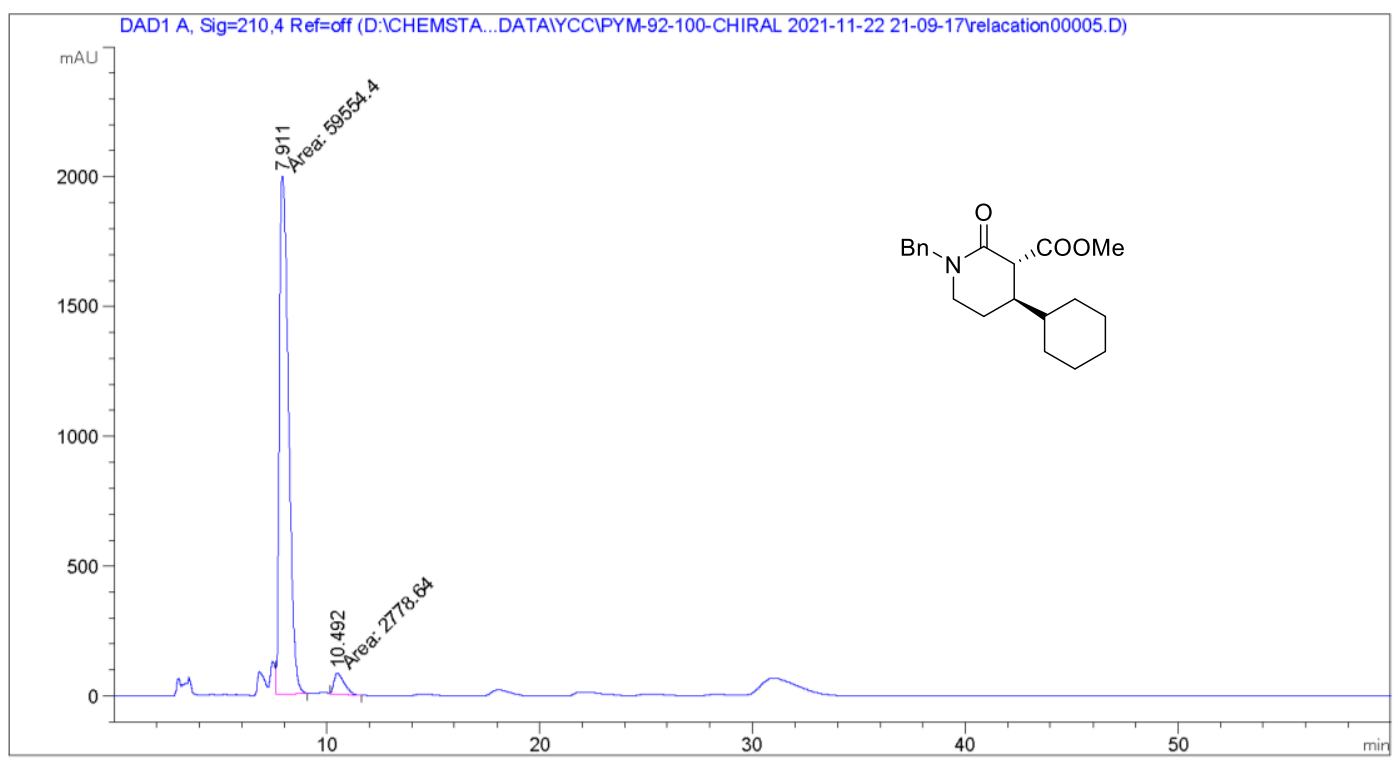

Area Percent Report

\begin{tabular}{|c|c|c|c|c|}
\hline Sorted By & : & Signal & & \\
\hline Multiplier & : & 1.0000 & & \\
\hline Dilution & $:$ & 1.0000 & & \\
\hline \multicolumn{5}{|c|}{ Use Multiplier \& Dilution Factor with ISTDs } \\
\hline \multicolumn{5}{|c|}{ Signal 1: DAD1 A, Sig=210,4 Ref=off } \\
\hline $\begin{array}{l}\text { Peak RetTime Type } \\
\# \quad[\mathrm{~min}]\end{array}$ & $\begin{array}{l}\text { Width } \\
\text { [min] }\end{array}$ & $\begin{array}{c}\text { Area } \\
{[\mathrm{mAU} * \mathrm{~s}]}\end{array}$ & $\begin{array}{l}\text { Height } \\
\text { [mAU] }\end{array}$ & $\begin{array}{c}\text { Area } \\
\%\end{array}$ \\
\hline 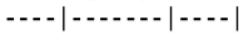 & $---\cdots \mid$ & |- & $|-\ldots-n|$ & 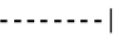 \\
\hline 17.911 FM & 0.4977 & $5.95544 \mathrm{e} 4$ & 1994.19617 & 95.5423 \\
\hline $210.492 \mathrm{FM}$ & 0.5588 & 2778.64331 & 82.86961 & 4.4577 \\
\hline
\end{tabular}




\section{Methyl (3R,4S)-1-methyl-2-oxo-4-phenylpiperidine-3-carboxylate 20}

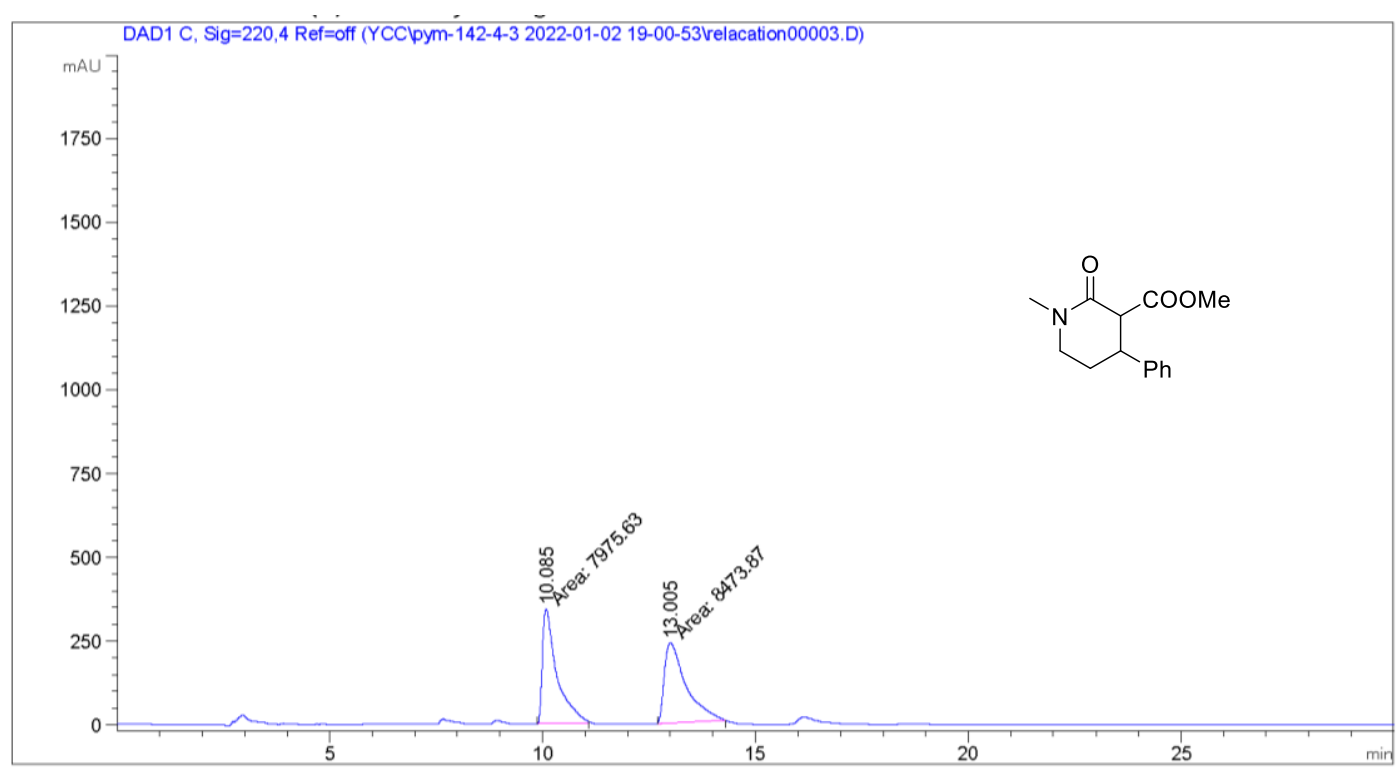

Area Percent Report

$\begin{array}{lll}\text { Sorted By } & : & \text { Signal } \\ \text { Multiplier } & : & 1.0000 \\ \text { Dilution } & : & 1.0000\end{array}$

Use Multiplier \& Dilution Factor with ISTDs

Signal 1: DAD1 C, Sig=220,4 Ref=off

\begin{tabular}{cccccc}
$\begin{array}{c}\text { Peak RetTime Type Width } \\
\text { \# }[\text { min] }\end{array}$ & $\begin{array}{c}\text { Area } \\
{[\mathrm{min}]}\end{array}\left[\begin{array}{c}\text { Height } \\
{[\mathrm{mAU} \text { s }]}\end{array}\right.$ & $\begin{array}{c}\text { Area } \\
{[\mathrm{mAU}]}\end{array}$ & $\%$ \\
\hline 1 & 10.085 MM & 0.3920 & 7975.62891 & 339.13190 & 48.4855 \\
2 & 13.005 MM & 0.5890 & 8473.87402 & 239.76608 & 51.5145
\end{tabular}




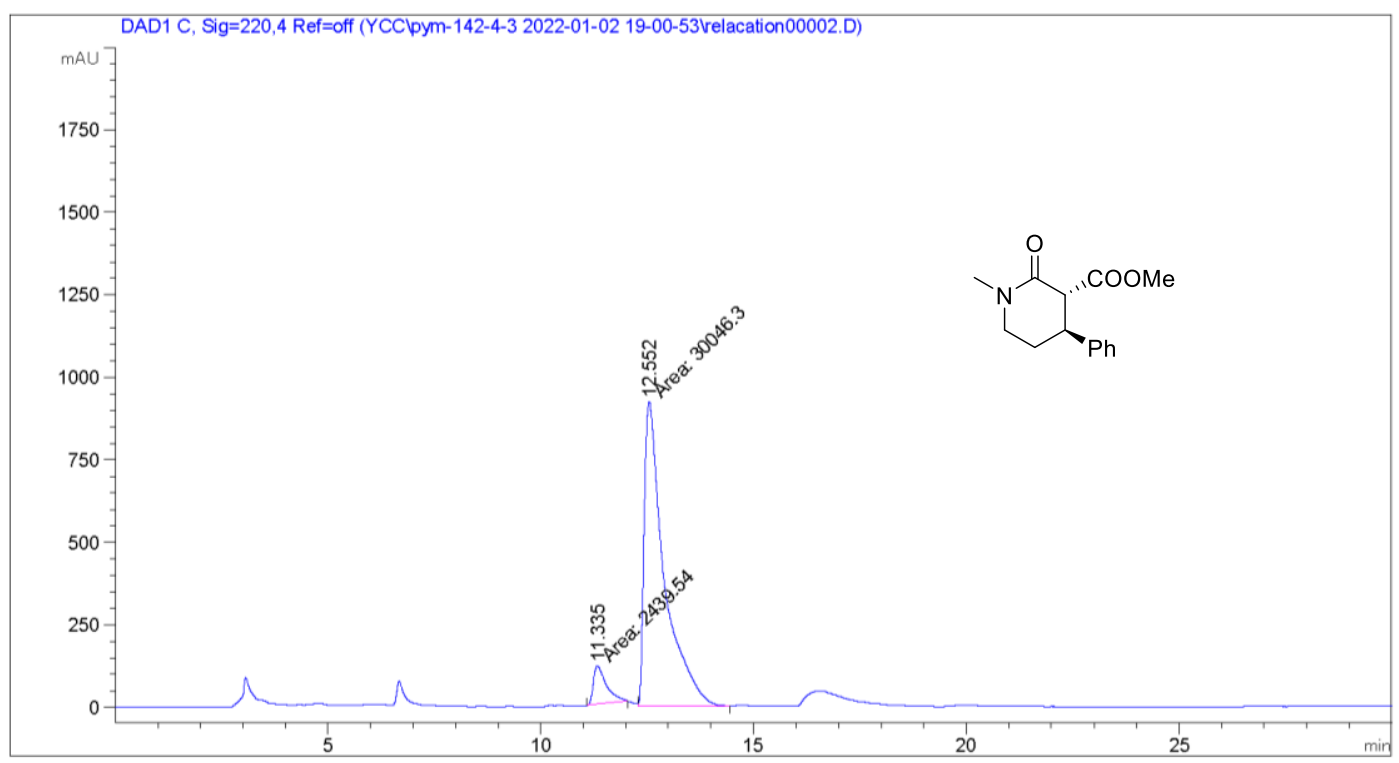

Area Percent Report

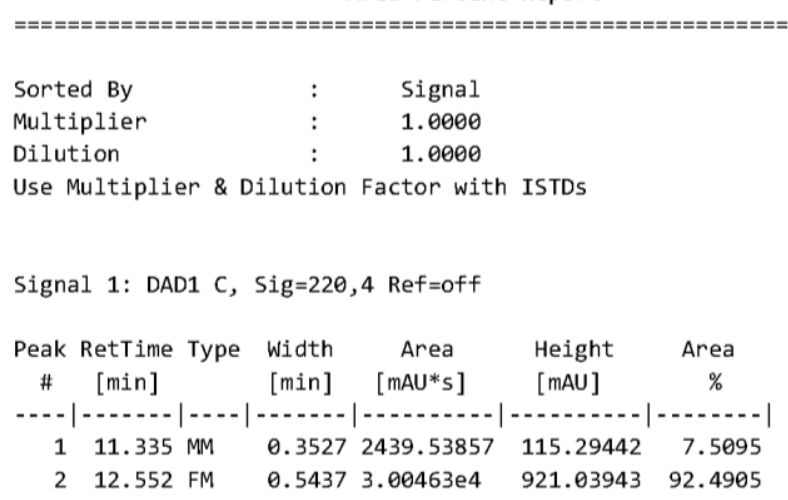


Methyl (3R,4R)-1-(4-methoxyphenyl)-3-methyl-2-oxo-4-phenylpiperidine-3-carboxylate

$3 \mathbf{a}$

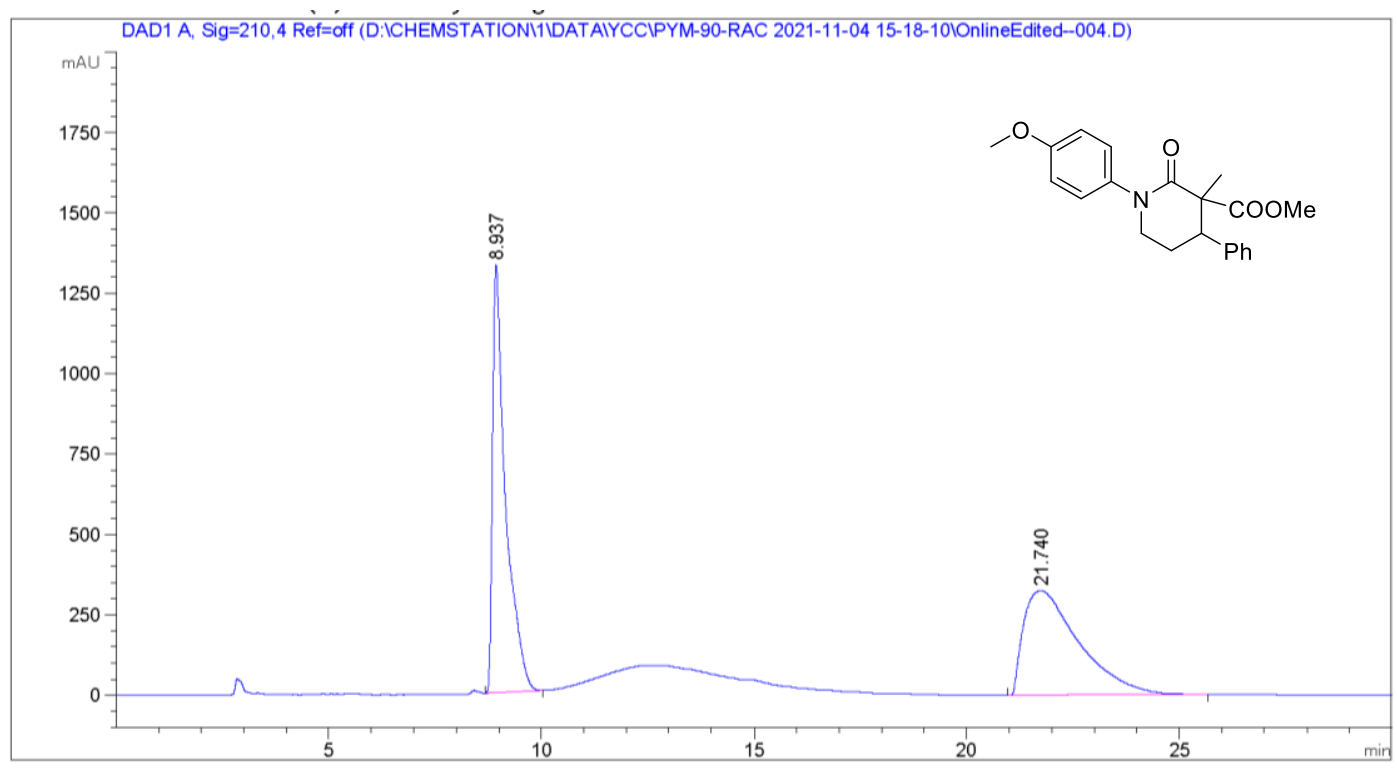

Area Percent Report

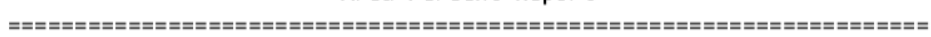

$\begin{array}{lll}\text { Sorted By } & : & \text { Signal } \\ \text { Multiplier } & : & 1.0000 \\ \text { Dilution } & : & 1.0000\end{array}$

Use Multiplier \& Dilution Factor with ISTDs

Signal 1: DAD1 A, Sig=210,4 Ref=off

Peak RetTime Type Width Area Height Area

\# [min] [min] [mAU*s] [mAU] \%

\begin{tabular}{ll|l|l|l|l|} 
& 8.937 VB & 0.3013 & $2.84549 \mathrm{e} 4$ & 1331.37109 & 49.3157
\end{tabular}

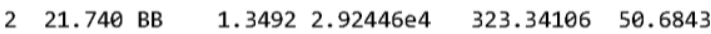

Totals : $\quad 5.76995 \mathrm{e} 4 \quad 1654.71216$ 


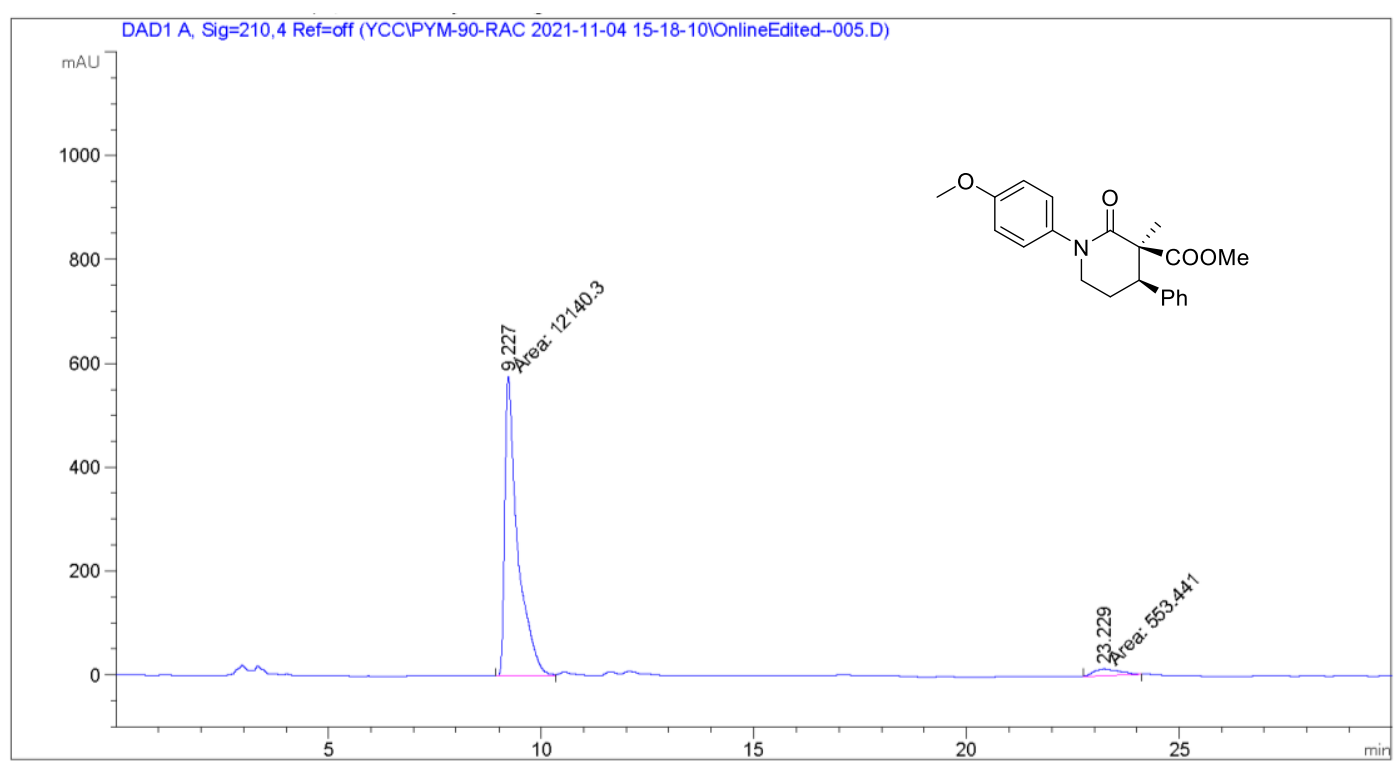

Area Percent Report

$\begin{array}{lll}\text { Sorted By } & : & \text { Signal } \\ \text { Multiplier } & : & 1.0000 \\ \text { Dilution } & : & 1.0000\end{array}$

Use Multiplier \& Dilution Factor with ISTDs

Signal 1: DAD1 A, Sig=210,4 Ref=off

\begin{tabular}{|c|c|c|c|c|c|c|}
\hline $\begin{array}{c}\text { Peak } \\
\#\end{array}$ & $\begin{array}{c}\text { RetTime } \\
\text { [min] }\end{array}$ & Type & $\begin{array}{l}\text { Width } \\
\text { [min] }\end{array}$ & $\begin{array}{c}\text { Area } \\
{\left[\mathrm{mAU}^{*} \mathrm{~s}\right]}\end{array}$ & $\begin{array}{l}\text { Height } \\
{[\mathrm{mAU}]}\end{array}$ & $\begin{array}{c}\text { Area } \\
\%\end{array}$ \\
\hline -... & | - . . . & & $-1 .-2$ & |-1-2] & | & 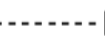 \\
\hline 1 & 9.227 & MF & 0.3511 & $1.21403 \mathrm{e} 4$ & 576.29443 & 95.6401 \\
\hline 2 & 23.229 & MM & 0.7288 & 553.44122 & 12.65698 & 4. 3599 \\
\hline Tota & : & & & $1.26938 \mathrm{e} 4$ & 588.95141 & \\
\hline
\end{tabular}


Methyl (3R,4R)-3-allyl-1-(4-methoxyphenyl)-2-oxo-4-phenylpiperidine-3-carboxylate 3b

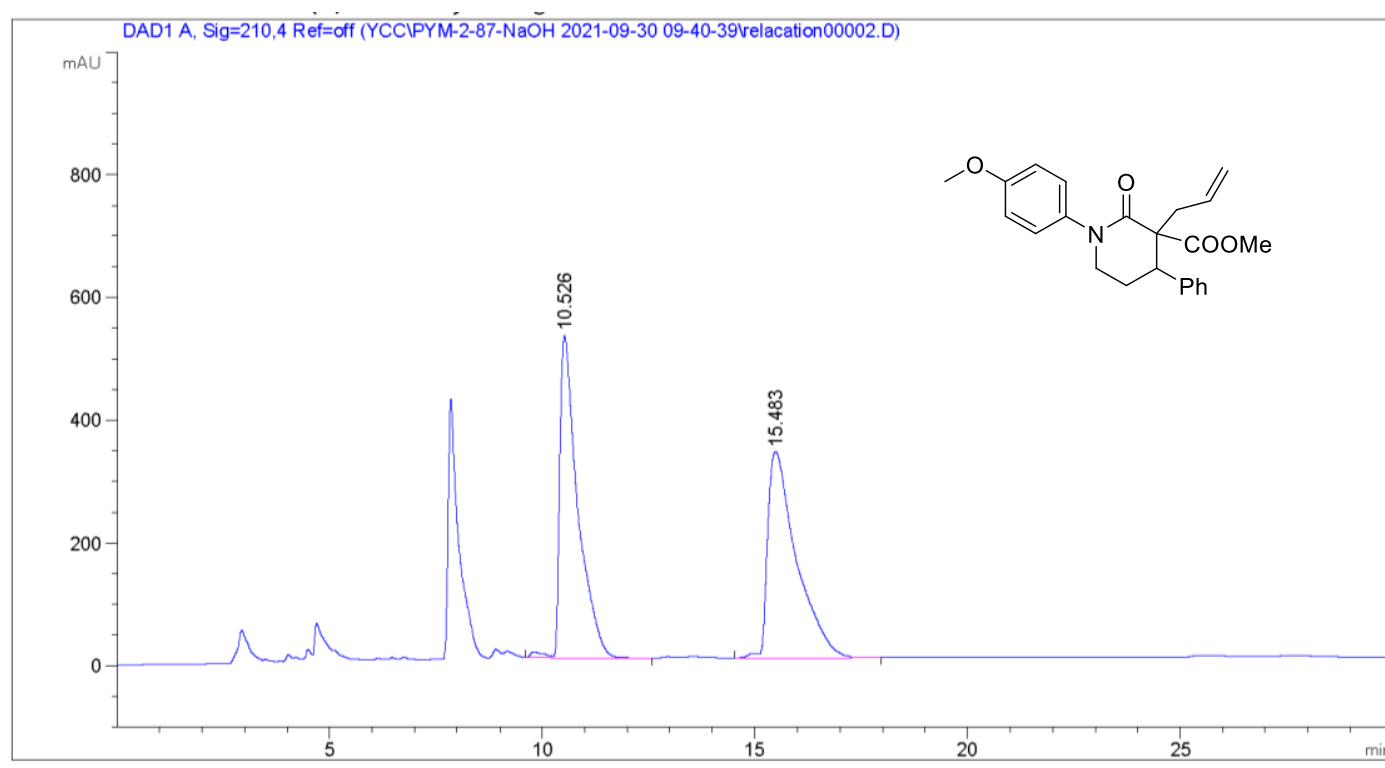

Area Percent Report

$\begin{array}{lll}\text { Sorted By } & : & \text { Signal } \\ \text { Multiplier } & : & 1.0000 \\ \text { Dilution } & : & 1.0000\end{array}$

Use Multiplier \& Dilution Factor with ISTDs

Signal 1: DAD1 A, Sig=210,4 Ref=off

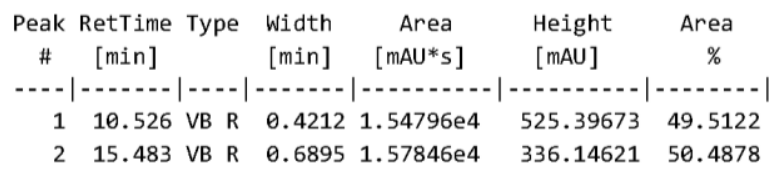




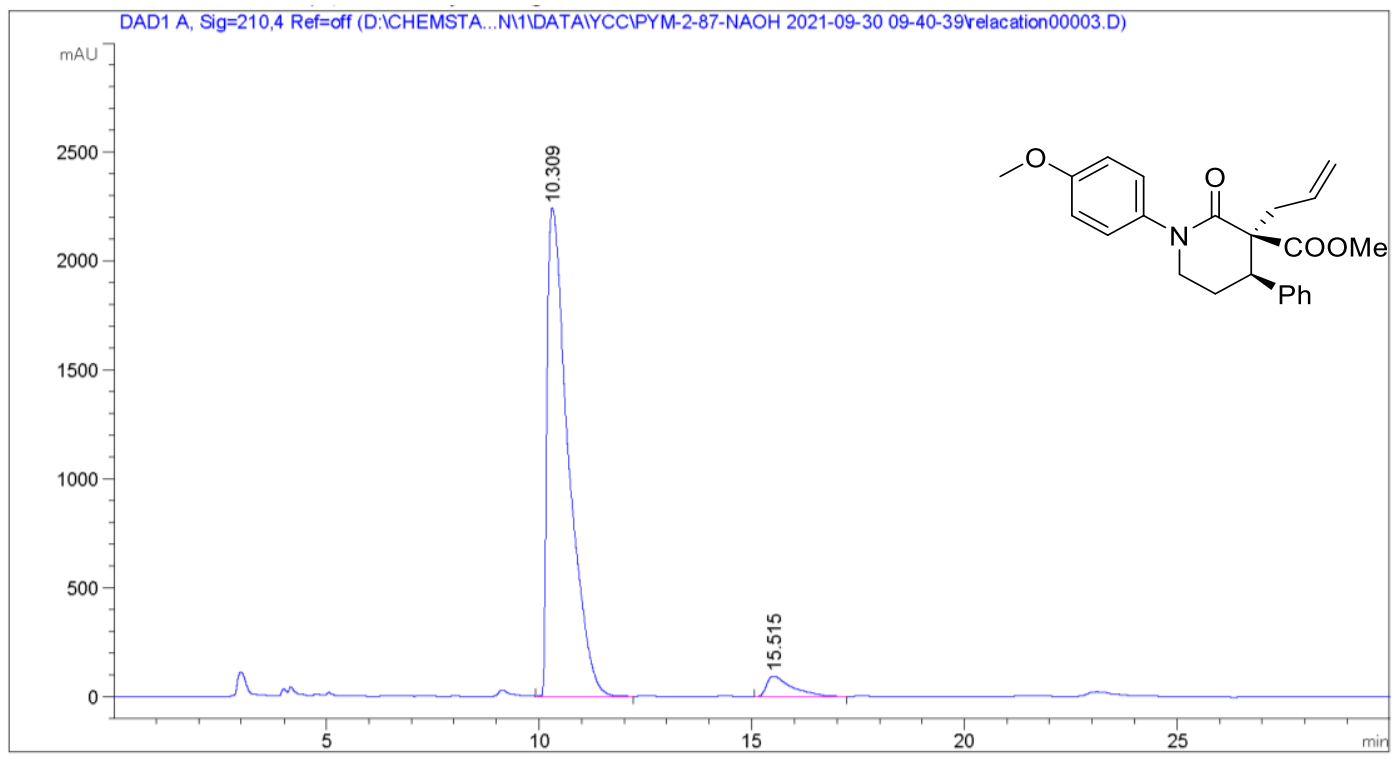

Area Percent Report

\begin{tabular}{|c|c|c|c|c|}
\hline Sorted By & $:$ & Signal & & \\
\hline Multiplier & : & 1.0000 & & \\
\hline Dilution & : & 1.0000 & & \\
\hline \multicolumn{5}{|c|}{ Use Multiplier \& Dilution Factor with ISTDs } \\
\hline \multicolumn{5}{|c|}{ Signal 1: DAD1 A, Sig=210,4 Ref=off } \\
\hline $\begin{array}{l}\text { Peak RetTime Type } \\
\# \quad[\text { min] }\end{array}$ & $\begin{array}{l}\text { Width } \\
\text { [min] }\end{array}$ & $\begin{array}{c}\text { Area } \\
{\left[\mathrm{mAU}^{*} \mathrm{~s}\right]}\end{array}$ & $\begin{array}{l}\text { Height } \\
{[\mathrm{mAU}]}\end{array}$ & $\begin{array}{c}\text { Area } \\
\%\end{array}$ \\
\hline$---|-\cdots|---\mid$ & $-\ldots$ & $|-\ldots-\ldots|$ & $|-\ldots-\ldots|$ & | \\
\hline 110.309 VB & 0.5171 & $7.75070 \mathrm{e} 4$ & 2243.01880 & 95.2406 \\
\hline $215.515 \mathrm{BB}$ & 0.5911 & 3873.17822 & 93.93089 & 4.7594 \\
\hline
\end{tabular}

LC 11/1/2021 7:40:08 PM SYSTEM

Page 1 of 2 
Methyl (3R,4R)-3-benzyl-1-(4-methoxyphenyl)-2-oxo-4-phenylpiperidine-3-carboxylate $3 c$

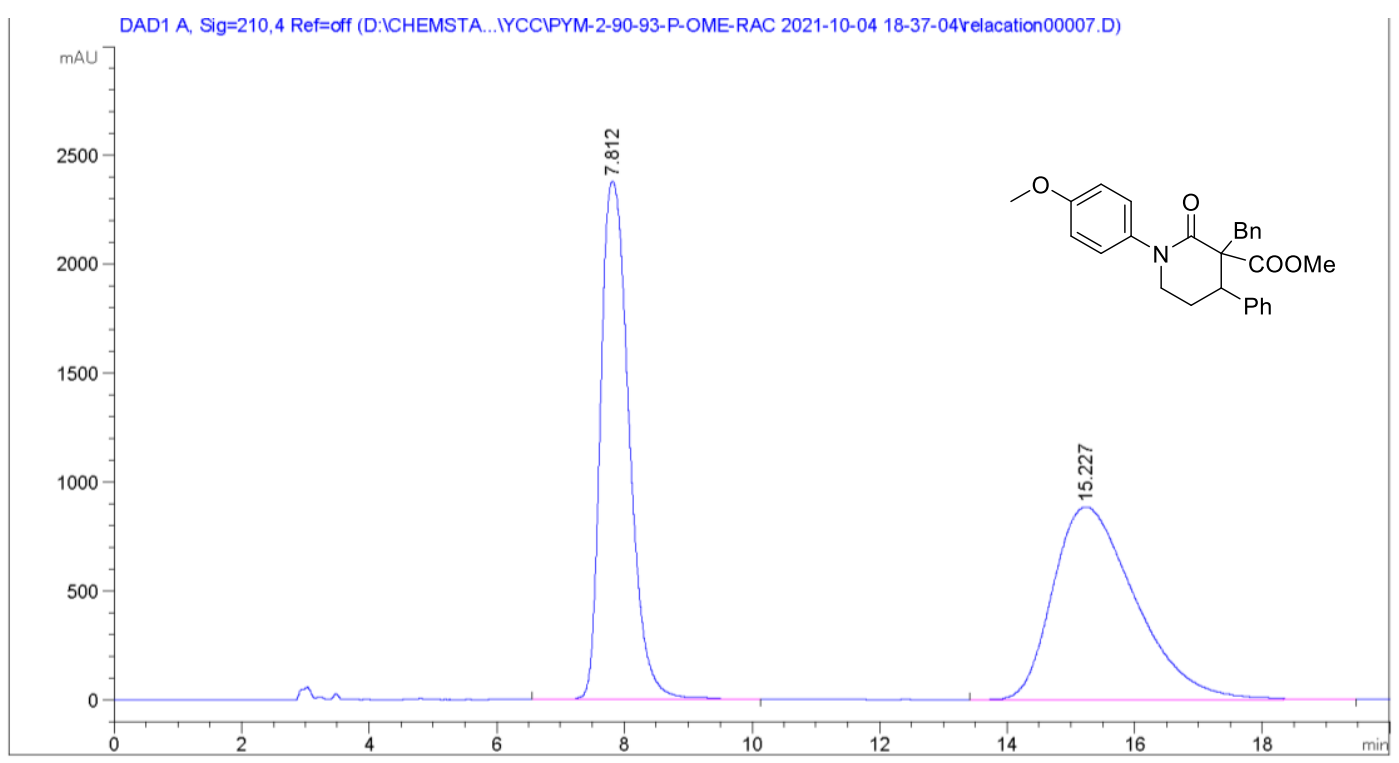

Area Percent Report

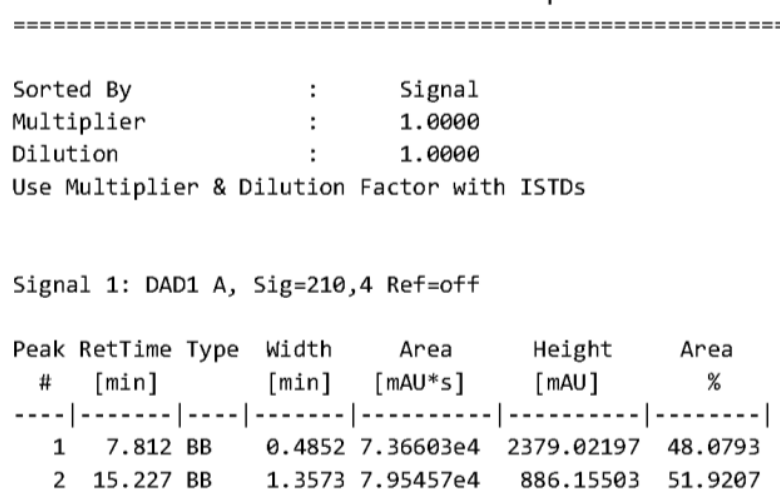




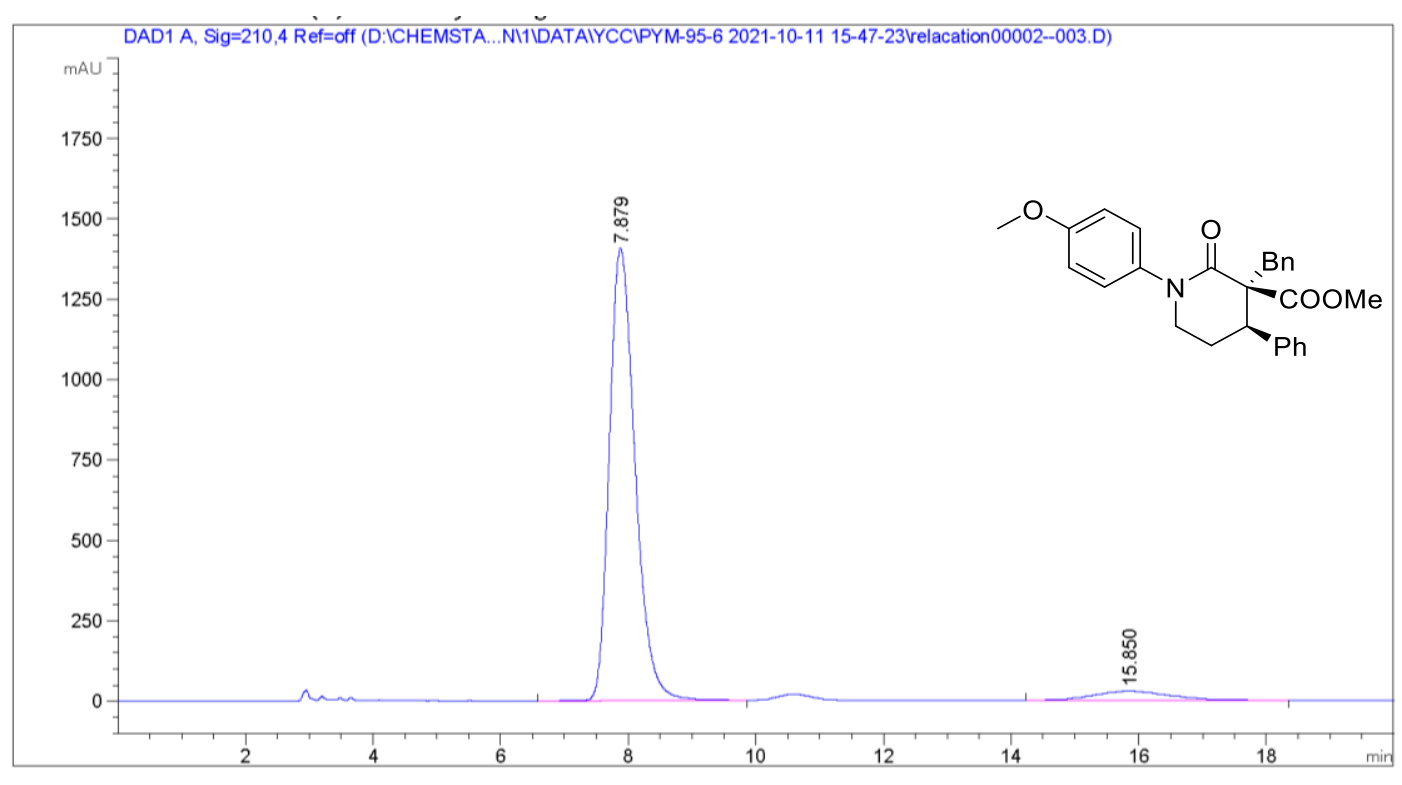

Area Percent Report

$\begin{array}{lll}\text { Sorted By } & : & \text { Signal } \\ \text { Multiplier } & : & 1.0000 \\ \text { Dilution } & : & 1.0000\end{array}$

Use Multiplier \& Dilution Factor with ISTDs

Signal 1: DAD1 A, Sig=210,4 Ref=off

\begin{tabular}{|c|c|c|c|c|c|}
\hline $\begin{array}{c}\text { Peak } \\
\#\end{array}$ & $\begin{array}{l}\text { RetTime Type } \\
\text { [min] }\end{array}$ & $\begin{array}{l}\text { Width } \\
\text { [min] }\end{array}$ & $\begin{array}{c}\text { Area } \\
{\left[\mathrm{mAU}^{*} \mathrm{~s}\right]}\end{array}$ & $\begin{array}{l}\text { Height } \\
{[\mathrm{mAU}]}\end{array}$ & $\begin{array}{c}\text { Area } \\
\%\end{array}$ \\
\hline$\ldots$ & $|-\ldots|$ & - & |-.... & $|-\ldots|$ & | \\
\hline 1 & 7.879 вB & 0.4354 & $3.94909 \mathrm{e} 4$ & 1407.01611 & 94.3886 \\
\hline 2 & $15.850 \mathrm{BB}$ & 1.0286 & 2347.71558 & 28.21882 & 5.6114 \\
\hline Tota & 1s : & & $4.18386 \mathrm{e} 4$ & 1435.23493 & \\
\hline
\end{tabular}




\section{Reference:}

1. Hughes, G.; Kimura, M.; Buchwald, S. L., Catalytic Enantioselective Conjugate Reduction of Lactones and Lactams. J. Am. Chem. Soc. 2003, 125, 11253-11258.

2. Khan, H. A.; Kou, K. G. M.; Dong, V. M., Nitrogen-directed ketone hydroacylation: Enantioselective synthesis of benzoxazecinones. Chem. Sci. 2011, 2, 407-410.

3. Estopiñá-Durán, S.; Donnelly, L. J.; Mclean, E. B.; Hockin, B. M.; Slawin, A. M. Z.; Taylor, J. E., Aryl Boronic Acid Catalysed Dehydrative Substitution of Benzylic Alcohols for $\mathrm{C}-\mathrm{O}$ Bond Formation. Chem. Eur. J. 2019, 25, 3950-3956.

4. Wu, J.; Qian, B.; Lu, L.; Yang, H.; Shang, Y.; Zhang, J., Access to the C2 C-H olefination, alkylation and deuteration of indoles by rhodium(iii) catalysis: an opportunity for diverse syntheses. Org. Chem. Front. 2021, 8, 3032-3040.

5. Yang, B.; Chansaenpak, K.; Wu, H.; Zhu, L.; Wang, M.; Li, Z.; Lu, H., Silver-promoted (radio)fluorination of unsaturated carbamates via a radical process. ChemComm 2017, 53, 3497-3500.

6. Wang, J.; Wang, Y.; Liu, D.; Zhang, W., Asymmetric Hydrogenation of $\beta$-Secondary Amino Ketones Catalyzed by a Ruthenocenyl Phosphino-oxazoline-ruthenium Complex (RuPHOX-Ru): the Synthesis of $\gamma$-Secondary Amino Alcohols. Adv. Synth. 2015, 357, 3262-3272. 